\title{
Total Synthesis of the Proposed Structure of Marineosin A
}

\author{
Bing Xu, ${ }^{a}$ Guang $\mathrm{Li}^{\mathrm{a}},{ }^{\mathrm{Jing} \mathrm{Li}},{ }^{\mathrm{a}}$ and Yian Shi*,a,b \\ ${ }^{a}$ Beijing National Laboratory for Molecular Sciences, CAS Key Laboratory of \\ Molecular Recognition and Function, Institute of Chemistry, Chinese Academy \\ of Sciences, Beijing 100190, China. \\ ${ }^{b}$ Department of Chemistry, Colorado State University, Fort Collins, Colorado \\ 80523, United States.
}

\section{Supporting Information}

Table of Contents

Experimental procedures and characterization data

S-2

$\mathrm{X}$-ray structures of $1,12,15,18$, and 19

S-18

NMR spectra

S-86 
General Methods. All commercially available reagents were used without further purification. All dry solvents were freshly distilled under nitrogen from appropriate drying agents before use. Tetrahydrofuran, 1,4-dioxane, and ethyl ether were distilled from sodium-benzophenone. Dichloromethane and acetonitrile were distilled from $\mathrm{CaH}_{2}$. Column chromatography was performed on silica gel (200-300 mesh). ${ }^{1}$ H NMR spectra were recorded on a $400 \mathrm{MHz}$ NMR spectrometer and ${ }^{13} \mathrm{C}$ NMR spectra were recorded on a $100 \mathrm{MHz}$ NMR spectrometer. IR spectra were recorded on a FT-IR spectrometer. Melting points were uncorrected.

\section{Preparation of compound 9}

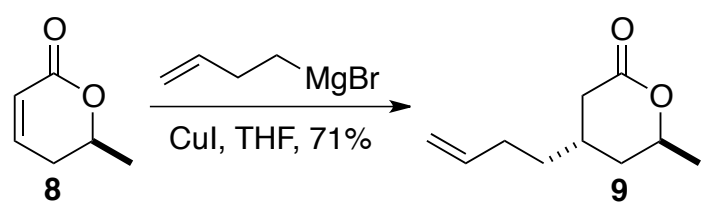

To a suspension of CuI $(4.80 \mathrm{~g}, 25.2 \mathrm{mmol})$ in anhydrous THF $(60 \mathrm{~mL})$ was added 3-butenylmagnesium bromide $\left(0.5 \mathrm{M}\right.$ solution in THF) $(201.0 \mathrm{~mL}, 100.5 \mathrm{mmol})$ at $-78{ }^{\circ} \mathrm{C}$ under $\mathrm{N}_{2}$. After the reaction mixture was stirred at $-35{ }^{\circ} \mathrm{C}$ for $30 \mathrm{~min}$, a solution of lactone $\mathbf{8}^{1-3}$ (5.65 g, $\left.50.4 \mathrm{mmol}\right)$ in THF $(30 \mathrm{~mL})$ was added via syringe pump over $3 \mathrm{~h}$. The resulting mixture was stirred at the same temperature for $1 \mathrm{~h}$, quenched with saturated aqueous $\mathrm{NH}_{4} \mathrm{Cl}$ solution $(50 \mathrm{~mL})$, extracted with EtOAc $(80 \mathrm{~mL} \times 3)$, washed with water and brine, dried $\left(\mathrm{MgSO}_{4}\right)$, filtered, concentrated, and purified by column chromatography (silica gel, petroleum ether:ethyl acetate $=5: 1)$ to afford compound 9 as a yellow oil $(6.0 \mathrm{~g}, 71 \%){ }^{4}$ $[\alpha]_{\mathrm{D}}^{25}=-46.5\left(c 1.03, \mathrm{CHCl}_{3}\right) ; \quad$ IR (film): $1740,1250,1092 \mathrm{~cm}^{-1} ; \quad{ }^{1} \mathrm{H}$ NMR $(400 \mathrm{MHz}$, $\left.\mathrm{CDCl}_{3}\right) \delta 5.82-5.70(\mathrm{~m}, 1 \mathrm{H}), 5.07-4.94(\mathrm{~m}, 2 \mathrm{H}), 4.58-4.46(\mathrm{~m}, 1 \mathrm{H}), 2.57(\mathrm{dd}, J=16.4,5.6 \mathrm{~Hz}$, $1 \mathrm{H}), 2.16(\mathrm{dd}, J=16.4,9.6 \mathrm{~Hz}, 1 \mathrm{H}), 2.12-2.00(\mathrm{~m}, 3 \mathrm{H}), 1.79-1.69(\mathrm{~m}, 1 \mathrm{H}), 1.68-1.58(\mathrm{~m}, 1 \mathrm{H})$, $1.47(\mathrm{q}, J=7.6 \mathrm{~Hz}, 2 \mathrm{H}), 1.35(\mathrm{~d}, J=6.4 \mathrm{~Hz}, 3 \mathrm{H}) ;{ }^{13} \mathrm{C} \mathrm{NMR}\left(100 \mathrm{MHz}, \mathrm{CDCl}_{3}\right) \delta$ 172.6, 137.7, 115.5, 73.8, 35.7, 35.2, 35.0, 31.0, 28.3, 21.5; HRMS calcd for $\mathrm{C}_{10} \mathrm{H}_{16} \mathrm{O}_{2} \mathrm{Na}[\mathrm{M}+\mathrm{Na}]$ : 191.1043; found: 191.1048 .

1) L. Shao, H. Kawano, M. Saburi, Y. Uchida, Tetrahedron 1993, 49, 1997. 
2) M. Wolberg, W. Hummel, M. Müller, Chem. Eur. J. 2001, 7, 4562.

3) Y. S. Park, C. I. Grove, M. González-López, S. Urgaonkar, J. C. Fettinger, J. T. Shaw, Angew. Chem. Int. Ed. 2011, 50, 3730.

4) D. Kim, J. Lee, P. J. Shim, J. I. Lim, T. Doi, S. Kim, J. Org. Chem. 2002, 67, 772.

\section{Preparation of compound 10}

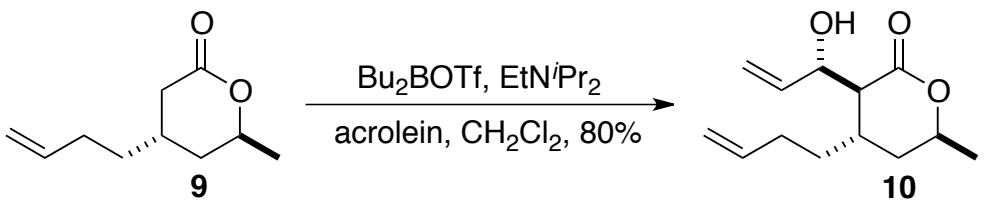

To a solution of compound $9(3.0 \mathrm{~g}, 17.8 \mathrm{mmol})$ in $\mathrm{CH}_{2} \mathrm{Cl}_{2}(60 \mathrm{~mL})$ were added $\mathrm{Bu}_{2} \mathrm{BOTf}\left(1 \mathrm{M}\right.$ in $\left.\mathrm{CH}_{2} \mathrm{Cl}_{2}\right)(35.7 \mathrm{~mL}, 35.7 \mathrm{mmol})$ and $\mathrm{EtN}^{i} \operatorname{Pr}_{2}(5.75 \mathrm{~g}, 44.5 \mathrm{mmol})$ at $-78^{\circ} \mathrm{C}$ under $\mathrm{N}_{2}$. After the mixture was stirred for $2 \mathrm{~h}$, acrolein $(2.5 \mathrm{~g}, 44.5 \mathrm{mmol})$ was added. The resulting mixture was stirred at $-78{ }^{\circ} \mathrm{C}$ for $1 \mathrm{~h}$ and at $0{ }^{\circ} \mathrm{C}$ for $2 \mathrm{~h}$. Upon quenching with $0.1 \mathrm{M}$ phosphate buffer $(\mathrm{pH} 6.8)(50 \mathrm{~mL}), \mathrm{MeOH}(100 \mathrm{~mL})$, and $\mathrm{H}_{2} \mathrm{O}_{2}(50 \mathrm{~mL})$, the reaction mixture was stirred at room temperature overnight, extracted with $\mathrm{CH}_{2} \mathrm{Cl}_{2}(50 \mathrm{~mL} \times 3)$, washed with water $(50 \mathrm{~mL} \times 3)$ and brine $(50 \mathrm{~mL} \times 3)$, dried $\left(\mathrm{Na}_{2} \mathrm{SO}_{4}\right)$, filtered, concentrated, and purified by column chromatography (silica gel, petroleum ether:ethyl acetate $=5: 1$ ) to afford alcohol 10 as a light yellow oil $(3.20 \mathrm{~g}, 80 \%) . \quad[\alpha]_{\mathrm{D}}{ }^{25}=-33.0\left(c 1.01, \mathrm{CHCl}_{3}\right) ; \quad$ IR (film): 3435, 1718, 1200, $1097 \mathrm{~cm}^{-1} ; \quad{ }^{1} \mathrm{H} \mathrm{NMR}\left(400 \mathrm{MHz}, \mathrm{CDCl}_{3}\right) \delta 6.02(\mathrm{ddd}, J=16.8$, 10.4, $6.4 \mathrm{~Hz}, 1 \mathrm{H}), 5.82-5.70(\mathrm{~m}, 1 \mathrm{H}), 5.28(\mathrm{dt}, J=17.2,1.2 \mathrm{~Hz}, 1 \mathrm{H}), 5.18(\mathrm{dt}, J=10.4,1.2$ $\mathrm{Hz}, 1 \mathrm{H}), 5.06-4.92(\mathrm{~m}, 2 \mathrm{H}), 4.50-4.38(\mathrm{~m}, 1 \mathrm{H}), 4.32-4.22(\mathrm{~m}, 1 \mathrm{H}), 3.41(\mathrm{~d}, J=7.6 \mathrm{~Hz}, 1 \mathrm{H})$, 2.27 (dd, $J=7.6,4.4 \mathrm{~Hz}, 1 \mathrm{H}), 2.20-1.96(\mathrm{~m}, 3 \mathrm{H}), 1.74-1.61(\mathrm{~m}, 3 \mathrm{H}), 1.48-1.37(\mathrm{~m}, 1 \mathrm{H}), 1.34$ $(\mathrm{d}, J=6.4 \mathrm{~Hz}, 3 \mathrm{H}) ; \quad{ }^{13} \mathrm{C} \mathrm{NMR}\left(100 \mathrm{MHz}, \mathrm{CDCl}_{3}\right) \delta 174.5,139.0,137.6,116.6,115.6,73.1$, 72.4, 50.2, 34.8, 33.9, 31.04, 31.00, 21.2; HRMS calcd for $\mathrm{C}_{13} \mathrm{H}_{20} \mathrm{O}_{3} \mathrm{Na}[\mathrm{M}+\mathrm{Na}]$ : 247.1305; found: 247.1305.

H. Ito, T. Momose, M. Konishi, E. Yamada, K. Watanabe, K. Iguchi, Tetrahedron 2006, 62, 10425. 


\section{Preparation of compound 7}
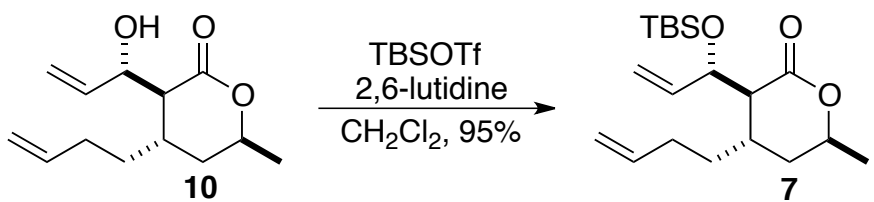

To a solution of alcohol $10(3.80 \mathrm{~g}, 16.9 \mathrm{mmol})$ and 2,6-lutidine $(2.72 \mathrm{~g}, 25.4 \mathrm{mmol})$ in dry $\mathrm{CH}_{2} \mathrm{Cl}_{2}(60 \mathrm{~mL})$ was added TBSOTf $(4.93 \mathrm{~g}, 18.6 \mathrm{mmol})$ dropwise at $0{ }^{\circ} \mathrm{C}$. The reaction mixture was stirred at room temperature for $0.5 \mathrm{~h}$, concentrated, and purified by column chromatography ( light yellow oil $(5.50 \mathrm{~g}, 95 \%) . \quad[\alpha]_{\mathrm{D}}^{25}=-38.4\left(c 1.22, \mathrm{CHCl}_{3}\right) ; \quad$ IR (film): 1725, 1253, $1096 \mathrm{~cm}^{-1} ; \quad{ }^{1} \mathrm{H}$ NMR $\left(400 \mathrm{MHz}, \mathrm{CDCl}_{3}\right) \delta 6.88(\mathrm{ddd}, J=17.2,10.4,4.8 \mathrm{~Hz}, 1 \mathrm{H}), 5.82-5.70$ (m, 1H), 5.31 (d, $J=16.8 \mathrm{~Hz}, 1 \mathrm{H}), 5.16(\mathrm{~d}, J=10.4 \mathrm{~Hz}, 1 \mathrm{H}), 5.00(\mathrm{~d}, J=17.2 \mathrm{~Hz}, 1 \mathrm{H}), 4.96$ $(\mathrm{d}, J=10.4 \mathrm{~Hz}, 1 \mathrm{H}), 4.89$ (t, $J=4.4 \mathrm{~Hz}, 1 \mathrm{H}), 4.54-4.42(\mathrm{~m}, 1 \mathrm{H}), 2.46(\mathrm{t}, J=4.4 \mathrm{~Hz}, 1 \mathrm{H})$, 2.17-1.95 (m, 3H), 1.80-1.69 (m, 1H), 1.69-1.55 (m, 2H), 1.49-1.36 (m, 1H), $1.32(\mathrm{~d}, J=6.4$ $\mathrm{Hz}, 3 \mathrm{H}), 0.90$ (s, 9H), 0.07 (s, 3H), $0.04(\mathrm{~s}, 3 \mathrm{H}) ;{ }^{13} \mathrm{C} \mathrm{NMR}\left(100 \mathrm{MHz}, \mathrm{CDCl}_{3}\right) \delta 172.5$, $138.1,137.8,116.1,115.2,73.6,72.8,51.1,34.6,34.0,31.4,29.1,26.1,21.7,18.4,-4.6,-4.8$; HRMS calcd for $\mathrm{C}_{19} \mathrm{H}_{34} \mathrm{O}_{3} \mathrm{NaSi}[\mathrm{M}+\mathrm{Na}]: 361.2169 ;$ found: 361.2166 .

1) E. J. Corey, H. Cho, C. Rücker, D. H. Hua, Tetrahedron Lett. 1981, 22, 3455.

2) L. Duroure, T. Jousseaume, R. Aráoz, E. Barré, P. Retailleau, L. Chabaud, J. Molgó, C. Guillou, Org. Biomol. Chem. 2011, 9, 8112.

\section{Preparation of compound 11}

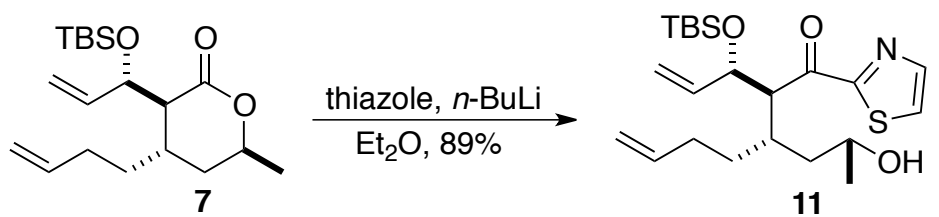

To a solution of thiazole $(1.66 \mathrm{~g}, 19.5 \mathrm{mmol})$ in ether $(80 \mathrm{~mL})$ was added $n$-BuLi $(2.4 \mathrm{M}$ in hexane) $(8.1 \mathrm{~mL}, 19.4 \mathrm{mmol})$ at $-78^{\circ} \mathrm{C}$ under $\mathrm{N}_{2}$. After the mixture was stirred at $-78^{\circ} \mathrm{C}$ for $1 \mathrm{~h}$, a solution of compound $7(5.50 \mathrm{~g}, 16.2 \mathrm{mmol})$ in ether $(20 \mathrm{~mL})$ was added. The 
reaction mixture was warmed to $-35^{\circ} \mathrm{C}$, stirred at this temperature for $5 \mathrm{~h}$, quenched with saturated aqueous $\mathrm{NH}_{4} \mathrm{Cl}$ solution, extracted with EtOAc $(50 \mathrm{~mL} \times 3)$, washed with water (30 $\mathrm{mL} \times 3)$ and brine $(30 \mathrm{~mL} \times 3)$, dried $\left(\mathrm{MgSO}_{4}\right)$, filtered, concentrated, and purified by column chromatography (silica gel, petroleum ether:ethyl acetate $=10: 1$ ) to afford alcohol $\mathbf{1 1}$ as a yellow oil $(6.12 \mathrm{~g}, 89 \%) . \quad[\alpha]_{\mathrm{D}}{ }^{25}=+5.5\left(c 0.97, \mathrm{CHCl}_{3}\right) ; \quad \mathrm{IR}($ film): 3366, 1681, 1394, 1252, $1064 \mathrm{~cm}^{-1} ; \quad{ }^{1} \mathrm{H}$ NMR $\left(400 \mathrm{MHz}, \mathrm{CDCl}_{3}\right) \delta 7.98(\mathrm{~d}, J=2.8 \mathrm{~Hz}, 1 \mathrm{H}), 7.65(\mathrm{~d}, J=3.2$ $\mathrm{Hz}, 1 \mathrm{H}), 5.84$ (ddd, $J=17.6,10.0,8.4 \mathrm{~Hz}, 1 \mathrm{H}), 5.72-5.60(\mathrm{~m}, 1 \mathrm{H}), 5.30$ (d, $J=17.2 \mathrm{~Hz}, 1 \mathrm{H})$, $5.20(\mathrm{~d}, J=10.0 \mathrm{~Hz}, 1 \mathrm{H}), 4.92(\mathrm{~d}, J=17.2 \mathrm{~Hz}, 1 \mathrm{H}), 4.85(\mathrm{~d}, J=10.0 \mathrm{~Hz}, 1 \mathrm{H}), 4.69(\mathrm{t}, J=9.2$ $\mathrm{Hz}, 1 \mathrm{H}), 4.25-4.10$ (m, 2H), 3.68 (br s, 1H), 2.17-2.04 (m, 1H), 2.04-1.88 (m, 2H), 1.74-1.63 $(\mathrm{m}, 1 \mathrm{H}), 1.62-1.44(\mathrm{~m}, 2 \mathrm{H}), 1.42-1.30(\mathrm{~m}, 1 \mathrm{H}), 1.20(\mathrm{~d}, J=6.0 \mathrm{~Hz}, 3 \mathrm{H}), 0.67(\mathrm{~s}, 9 \mathrm{H}), 0.00(\mathrm{~s}$, 3H), -0.02 (s, 3H); ${ }^{13} \mathrm{C}$ NMR (100 MHz, $\left.\mathrm{CDCl}_{3}\right) \delta 194.1,168.7,144.5,140.1,138.5,126.5$, $117.5,115.0,73.7,65.0,53.7,40.8,34.7,31.8,29.7,25.8,23.2,18.1,-3.7,-5.1$; HRMS calcd for $\mathrm{C}_{22} \mathrm{H}_{37} \mathrm{O}_{3} \mathrm{NNaSSi}[\mathrm{M}+\mathrm{Na}]$ : 446.2156; found: 446.2153 .

A. Dondoni, D. Perrone, P. Merino, J. Chem. Soc., Chem. Commun. 1991, 1313.

\section{Preparation of compound 12}
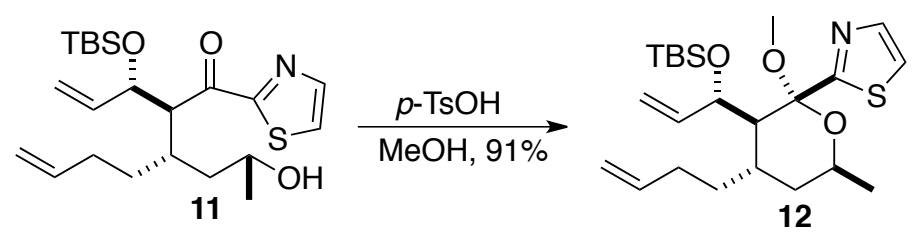

To a solution of alcohol 11 (1.36 g, $3.21 \mathrm{mmol})$ in $\mathrm{MeOH}(30 \mathrm{~mL})$ was added $p$-TsOH $(0.0829 \mathrm{~g}, 0.48 \mathrm{mmol})$. The reaction mixture was stirred at $\mathrm{rt}$ for $3 \mathrm{~h}$, quenched with $\mathrm{Et}_{3} \mathrm{~N}$, concentrated, and purified by column chromatography (silica gel, petroleum ether:ether = 10:1) to give compound 12 as a white solid $(1.27 \mathrm{~g}, 91 \%)$. mp. $55-56{ }^{\circ} \mathrm{C} ; \quad[\alpha]_{\mathrm{D}}{ }^{25}=-78.2(c$ 1.00, $\left.\mathrm{CHCl}_{3}\right) ; \quad$ IR (film): 1252, 1128, $1040 \mathrm{~cm}^{-1} ;{ }^{1} \mathrm{H}$ NMR (400 MHz, $\left.\mathrm{CDCl}_{3}\right) \delta 7.87(\mathrm{~d}$, $J=3.2 \mathrm{~Hz}, 1 \mathrm{H}), 7.32(\mathrm{~d}, J=3.2 \mathrm{~Hz}, 1 \mathrm{H}), 5.98(\mathrm{ddd}, J=17.2,10.8,3.2 \mathrm{~Hz}, 1 \mathrm{H}), 5.90-5.78(\mathrm{~m}$, $1 \mathrm{H}), 5.11(\mathrm{dt}, J=17.2,2.0, \mathrm{~Hz}, 1 \mathrm{H}), 5.04-4.86(\mathrm{~m}, 3 \mathrm{H}), 4.04-3.92(\mathrm{~m}, 1 \mathrm{H}), 3.70-3.62(\mathrm{~m}, 1 \mathrm{H})$, $2.95(\mathrm{~s}, 3 \mathrm{H}), 2.33(\mathrm{~d}, J=3.6 \mathrm{~Hz}, 1 \mathrm{H}), 2.20-2.00(\mathrm{~m}, 3 \mathrm{H}), 1.89-1.65$ (m, 3H), 1.30-1.24 (m, $1 \mathrm{H}), 1.24(\mathrm{~d}, J=6.0 \mathrm{~Hz}, 1 \mathrm{H}), 0.84(\mathrm{~s}, 9 \mathrm{H}),-0.02(\mathrm{~s}, 3 \mathrm{H}),-0.15(\mathrm{~s}, 3 \mathrm{H}) ;{ }^{13} \mathrm{C} \mathrm{NMR}(100 \mathrm{MHz}$, 
$\left.\mathrm{CDCl}_{3}\right) \delta 171.5,143.4,139.6,119.9,114.2,112.8,101.5,71.2,63.1,51.1,49.1,35.7,33.4$, $32.7,29.2,26.1,21.8,18.3,-4.8,-5.0 ;$ HRMS calcd for $\mathrm{C}_{23} \mathrm{H}_{39} \mathrm{O}_{3} \mathrm{NNaSSi}[\mathrm{M}+\mathrm{Na}]$ : 460.2312; found: 460.2307 .

G. Devianne, J.-M. Escudier, M. Baltas, L. Gorrichon, J. Org. Chem. 1995, 60, 7343.

\section{Preparation of compound 6}
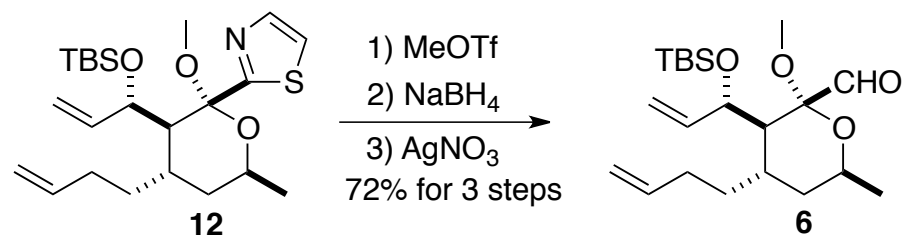

A mixture of compound $12(2.50 \mathrm{~g}, 5.72 \mathrm{mmol})$, activated $3 \AA \mathrm{MS}(6.00 \mathrm{~g})$, and anhydrous $\mathrm{CH}_{3} \mathrm{CN}(60 \mathrm{~mL})$ was stirred at room temperature for $10 \mathrm{~min}$, followed by the addition of MeOTf (1.88 $\mathrm{g}, 11.44 \mathrm{mmol})$. The resulting suspension was stirred at room temperature for $15 \mathrm{~min}$, concentrated, diluted with $\mathrm{CH}_{3} \mathrm{CH}_{2} \mathrm{OH}(60 \mathrm{~mL})$, and cooled to $0{ }^{\circ} \mathrm{C}$. At this point, $\mathrm{NaBH}_{4}(0.43 \mathrm{~g}, 11.44 \mathrm{mmol})$ was added. The reaction mixture was stirred at $0{ }^{\circ} \mathrm{C}$ for $30 \mathrm{~min}$ and at room temperature for an additional $30 \mathrm{~min}$, quenched with acetone, filtered through a plug of silica gel, concentrated, and dissolved in $\mathrm{CH}_{3} \mathrm{CN}(60 \mathrm{~mL})$ and $\mathrm{H}_{2} \mathrm{O}$ $(6 \mathrm{~mL}) . \quad \mathrm{AgNO}_{3}(1.46 \mathrm{~g}, 8.58 \mathrm{mmol})$ was added in one portion with vigorous stirring. The resulting mixture was stirred at room temperature for $10 \mathrm{~min}$, diluted with $0.1 \mathrm{M}$ phosphate buffer $\left(\mathrm{pH}\right.$ 6.8) $(50 \mathrm{~mL})$, extracted with $\mathrm{CH}_{2} \mathrm{Cl}_{2}(80 \mathrm{~mL} \times 3)$, dried $\left(\mathrm{Na}_{2} \mathrm{SO}_{4}\right)$, filtered, concentrated, and purified by column chromatography (silica gel, petroleum ether:ether = $50: 1$ to $20: 1)$ to give aldehyde 6 as a yellow oil $(1.57 \mathrm{~g}, 72 \%) . \quad[\alpha]_{\mathrm{D}}{ }^{25}=-14.2(\mathrm{c} 1.20$, $\left.\mathrm{CHCl}_{3}\right) ; \quad$ IR (film): $1745,1252,1036 \mathrm{~cm}^{-1} ;{ }^{1} \mathrm{H}$ NMR $\left(400 \mathrm{MHz}, \mathrm{CDCl}_{3}\right) \delta 9.25(\mathrm{~s}, 1 \mathrm{H})$, 5.86-5.69 (m, 2H), $5.13(\mathrm{~d}, J=11.2 \mathrm{~Hz}, 1 \mathrm{H}), 5.12$ (d, $J=16.8 \mathrm{~Hz}, 1 \mathrm{H}), 4.99$ (d, $J=17.2 \mathrm{~Hz}$, $1 \mathrm{H}), 4.93(\mathrm{~d}, J=10.4 \mathrm{~Hz}, 1 \mathrm{H}), 4.21(\mathrm{t}, J=8.0 \mathrm{~Hz}, 1 \mathrm{H}), 3.95-3.82(\mathrm{~m}, 1 \mathrm{H}), 3.13(\mathrm{~s}, 3 \mathrm{H})$, 2.10-2.00 (m, 2H), 1.92-1.75 (m, 3H), 1.65-1.49 (m, 2H), 1.35-1.27 (m, 1H), $1.26(\mathrm{~d}, J=6.0$ $\mathrm{Hz}, 3 \mathrm{H}), 0.86(\mathrm{~s}, 9 \mathrm{H}), 0.03(\mathrm{~s}, 3 \mathrm{H}), 0.01(\mathrm{~s}, 3 \mathrm{H}) ;{ }^{13} \mathrm{C} \mathrm{NMR}\left(100 \mathrm{MHz}, \mathrm{CDCl}_{3}\right) \delta 195.8$, $140.3,139.0,116.7,114.7,100.9,74.6,63.1,49.7,48.7,33.7,32.9,32.3,31.6,26.3,21.8$, 
18.5, -3.4, -4.1; HRMS calcd for $\mathrm{C}_{21} \mathrm{H}_{38} \mathrm{O}_{4} \mathrm{NaSi}[\mathrm{M}+\mathrm{Na}]: \quad 405.2432 ; \quad$ found: 405.2427 .

A. Dondoni, N. Catozzi, A. Marra, J. Org. Chem. 2004, 69, 5023.

\section{Preparation of compound 13}
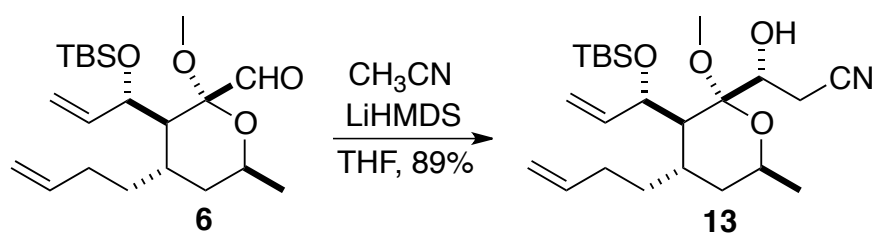

To a solution of $\mathrm{CH}_{3} \mathrm{CN}(1.73 \mathrm{~g}, 42.0 \mathrm{mmol})$ in THF $(40 \mathrm{~mL})$ was added LiHMDS (1.0 M solution in THF) $(42.0 \mathrm{~mL}, 42.0 \mathrm{mmol})$ at $-78{ }^{\circ} \mathrm{C}$ under $\mathrm{N}_{2}$. After the reaction mixture was stirred at $-78{ }^{\circ} \mathrm{C}$ for $1 \mathrm{~h}$, a solution of aldehyde $6(2.3 \mathrm{~g}, 6.0 \mathrm{mmol})$ in THF $(10 \mathrm{~mL})$ was added. The resulting mixture was stirred at $-78{ }^{\circ} \mathrm{C}$ for $5 \mathrm{~h}$, quenched with saturated aqueous $\mathrm{NH}_{4} \mathrm{Cl}$ solution, extracted with EtOAc $(50 \mathrm{~mL} \times 3)$, washed with water $(30 \mathrm{~mL} \times 3)$ and brine $(30 \mathrm{~mL} \times 3)$, dried $\left(\mathrm{MgSO}_{4}\right)$, filtered, concentrated, and purified by column chromatography ( silica gel, petroleum ether:ethyl acetate $=5: 1)$ to afford alcohol $\mathbf{1 3}$ as a colorless oil $(2.27 \mathrm{~g}$, $89 \%) . \quad[\alpha]_{\mathrm{D}}^{25}=-23.1\left(c 1.11, \mathrm{CHCl}_{3}\right) ; \quad$ IR (film): $3425,2253,1254 \mathrm{~cm}^{-1} ; \quad{ }^{1} \mathrm{H}$ NMR (400 $\left.\mathrm{MHz}, \mathrm{CDCl}_{3}\right) \delta 6.07(\mathrm{ddd}, J=17.2,10.0,7.6 \mathrm{~Hz}, 1 \mathrm{H}), 5.86-5.75(\mathrm{~m}, 1 \mathrm{H}), 5.16(\mathrm{~d}, J=16.8$ $\mathrm{Hz}, 1 \mathrm{H}), 5.10(\mathrm{~d}, J=10.4 \mathrm{~Hz}, 1 \mathrm{H}), 5.01(\mathrm{dd}, J=17.2,1.6 \mathrm{~Hz}, 1 \mathrm{H}), 4.96(\mathrm{~d}, J=10.0 \mathrm{~Hz}, 1 \mathrm{H})$, $4.46(\mathrm{dd}, J=7.2,4.8 \mathrm{~Hz}, 1 \mathrm{H}), 4.35$ (br s, 1H), 3.99-3.87 (m, 2H), 3.23 (s, 3H), 2.89 (dd, $J=$ 16.8, $2.8 \mathrm{~Hz}, 1 \mathrm{H}), 2.52(\mathrm{dd}, J=17.2,9.2 \mathrm{~Hz}, 1 \mathrm{H}), 2.19-2.07$ (m, 1H), 2.04-1.91 (m, 3H), 1.72-1.60 (m, 1H), 1.56-1.39 (m, 2H), 1.35-1.27 (m, 1H), $1.15(\mathrm{~d}, J=6.4 \mathrm{~Hz}, 3 \mathrm{H}), 0.91(\mathrm{~s}$, 9H), $0.12(\mathrm{~s}, 3 \mathrm{H}), 0.09$ (s, 3H); ${ }^{13} \mathrm{C}$ NMR (100 MHz, $\left.\mathrm{CDCl}_{3}\right) \delta 140.1,138.7,119.6,115.4$, $114.9,100.6,74.4,71.3,64.9,49.6,44.7,33.7,33.6,31.9,30.1,26.1,21.6,20.0,18.4,-3.5$, -4.6; HRMS calcd for $\mathrm{C}_{23} \mathrm{H}_{41} \mathrm{O}_{4} \mathrm{NNaSi}[\mathrm{M}+\mathrm{Na}]: \quad 446.2697$; found: 446.2695 .

F. F. Fleming, B. C. Shook, J. Org. Chem. 2002, 67, 3668. 


\section{Preparation of compound 13a}

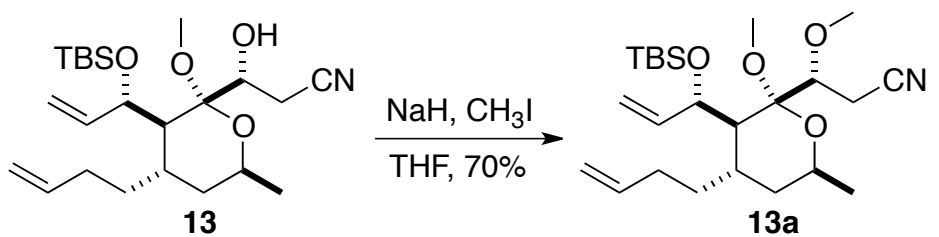

To a suspension of $\mathrm{NaH}(60 \%$ in mineral oil) $(0.14 \mathrm{~g}, 3.60 \mathrm{mmol})$ in THF $(50 \mathrm{~mL})$ was added a solution of alcohol $13(1.02 \mathrm{~g}, 2.40 \mathrm{mmol})$ in THF $(10 \mathrm{~mL})$ at $0{ }^{\circ} \mathrm{C}$ under $\mathrm{N}_{2}$. After the reaction mixture was stirred at $0{ }^{\circ} \mathrm{C}$ for $0.5 \mathrm{~h}, \mathrm{CH}_{3} \mathrm{I}(1.02 \mathrm{~g}, 7.20 \mathrm{mmol})$ was added. The resulting mixture was stirred at room temperature for $4 \mathrm{~h}$, quenched with saturated aqueous $\mathrm{NH}_{4} \mathrm{Cl}$ solution, extracted with EtOAc $(50 \mathrm{~mL} \times 3)$, washed with water $(30 \mathrm{~mL} \times 3)$ and brine $(30 \mathrm{~mL} \times 3)$, dried $\left(\mathrm{MgSO}_{4}\right)$, filtered, concentrated, and purified by column chromatography ( silica gel, petroleum ether:ether $=50: 1$ to $10: 1$ ) to afford compound 13a as a colorless oil $(0.73 \mathrm{~g}, 70 \%) . \quad[\alpha]_{\mathrm{D}}{ }^{25}=-67.8\left(c 1.10, \mathrm{CHCl}_{3}\right) ; \quad$ IR (film): $2251,1254 \mathrm{~cm}^{-1} ; \quad{ }^{1} \mathrm{H}$ NMR $\left(400 \mathrm{MHz}, \mathrm{CDCl}_{3}\right) \delta 6.06(\mathrm{ddd}, J=17.2,10.8,2.8 \mathrm{~Hz}, 1 \mathrm{H}), 5.88-5.74(\mathrm{~m}, 1 \mathrm{H}), 5.28(\mathrm{~d}, J=$ $16.8 \mathrm{~Hz}, 1 \mathrm{H}), 5.07$ (d, $J=10.4 \mathrm{~Hz}, 1 \mathrm{H}), 4.98(\mathrm{dd}, J=17.2,1.2 \mathrm{~Hz}, 1 \mathrm{H}), 4.91(\mathrm{~d}, J=10.0 \mathrm{~Hz}$, 1H), 4.55-4.48 (m, 1H), 3.91-3.79 (m, 1H), $3.60(\mathrm{dd}, J=8.0,3.2 \mathrm{~Hz}, 1 \mathrm{H}), 3.56(\mathrm{~s}, 3 \mathrm{H}), 3.26$ (s, 3H), $2.90(\mathrm{dd}, J=17.2,3.2 \mathrm{~Hz}, 1 \mathrm{H}), 2.55(\mathrm{dd}, J=17.2,8.4 \mathrm{~Hz}, 1 \mathrm{H}), 2.14-1.94(\mathrm{~m}, 3 \mathrm{H})$, $1.86-1.80(\mathrm{~m}, 1 \mathrm{H}), 1.72-1.58(\mathrm{~m}, 2 \mathrm{H}), 1.56-1.46(\mathrm{~m}, 1 \mathrm{H}), 1.22-1.15(\mathrm{~m}, 1 \mathrm{H}), 1.12(\mathrm{~d}, J=6.0$ $\mathrm{Hz}, 3 \mathrm{H}), 0.92$ (s, 9H), 0.06 (s, 3H), 0.04 (s, 3H); ${ }^{13} \mathrm{C}$ NMR (100 MHz, $\left.\mathrm{CDCl}_{3}\right) \delta 140.1$, $139.3,119.1,114.3,113.3,100.6,81.1,70.4,63.0,59.9,49.9,45.8,35.4,32.9,32.8,28.8$, 26.1, 21.9, 18.6, 18.4, -4.5, -4.6; HRMS calcd for $\mathrm{C}_{24} \mathrm{H}_{43} \mathrm{O}_{4} \mathrm{NNaSi}[\mathrm{M}+\mathrm{Na}]$ : 460.2854 ; found: 460.2849 .

X.-C. Cai, X. Wu, B. B. Snider, Org. Lett. 2010, 12, 1600. 


\section{Preparation of compound 14}
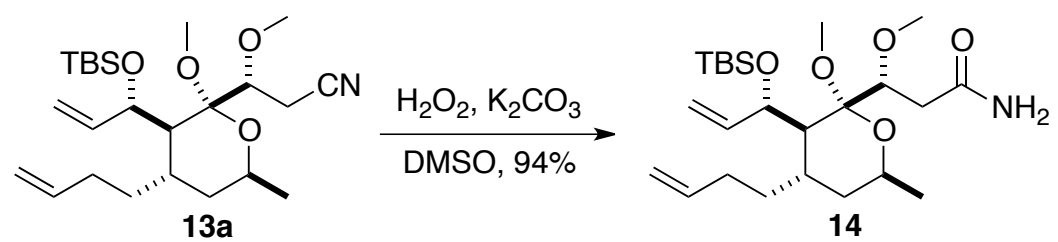

To a solution of compound 13a (2.00 g, $4.57 \mathrm{mmol})$ in DMSO $(80 \mathrm{~mL})$ were added $30 \%$ aqueous $\mathrm{H}_{2} \mathrm{O}_{2}(5.18 \mathrm{~g}, 45.7 \mathrm{mmol})$ and $\mathrm{K}_{2} \mathrm{CO}_{3}(1.26 \mathrm{~g}, 9.14 \mathrm{mmol})$ at $25{ }^{\circ} \mathrm{C}$. The reaction mixture was stirred at $25{ }^{\circ} \mathrm{C}$ overnight, diluted with water $(100 \mathrm{~mL})$, extracted with EtOAc $(60 \mathrm{~mL} \times 3)$, washed with water $(30 \mathrm{~mL} \times 3)$ and brine $(30 \mathrm{~mL} \times 3)$, dried $\left(\mathrm{MgSO}_{4}\right)$, filtered, concentrated, and purified by column chromatography (silica gel, petroleum ether:ethyl acetate $=1: 1)$ to afford amide 14 as a colorless oil $(1.95 \mathrm{~g}, 94 \%) . \quad[\alpha]_{\mathrm{D}}{ }^{25}=-68.7(c 1.01$, $\left.\mathrm{CHCl}_{3}\right) ; \quad$ IR (film): 3332, 3193, 1673, $1252 \mathrm{~cm}^{-1} ;{ }^{1} \mathrm{H}$ NMR $\left(400 \mathrm{MHz}, \mathrm{CDCl}_{3}\right) \delta 6.12$ (ddd, $J=17.2,10.4,2.4 \mathrm{~Hz}, 1 \mathrm{H}), 5.87-5.68(\mathrm{~m}, 3 \mathrm{H}), 5.26(\mathrm{~d}, J=17.2 \mathrm{~Hz}, 1 \mathrm{H}), 5.04(\mathrm{~d}, J=$ $10.8 \mathrm{~Hz}, 1 \mathrm{H}), 4.97(\mathrm{~d}, J=17.2 \mathrm{~Hz}, 1 \mathrm{H}), 4.90(\mathrm{~d}, J=10.0 \mathrm{~Hz}, 1 \mathrm{H}), 4.68-4.61(\mathrm{~m}, 1 \mathrm{H})$, 3.86-3.73 (m, 2H), 3.47 (s, 3H), $3.20(\mathrm{~s}, 3 \mathrm{H}), 2.78(\mathrm{dd}, J=14.8,2.4 \mathrm{~Hz}, 1 \mathrm{H}), 2.32$ (dd, $J=$ $15.2,8.8 \mathrm{~Hz}, 1 \mathrm{H}), 2.12-1.97$ (m, 3H), 1.79-1.73 (m, 1H), 1.73-1.59 (m, 2H), 1.59-1.48 (m, 1H), 1.21-1.14 (m, 1H), 1.12 (d, $J=6.4 \mathrm{~Hz}, 3 \mathrm{H}), 0.92(\mathrm{~s}, 9 \mathrm{H}), 0.06(\mathrm{~s}, 3 \mathrm{H}), 0.03$ (s, 3H); ${ }^{13} \mathrm{C} \mathrm{NMR}\left(100 \mathrm{MHz}, \mathrm{CDCl}_{3}\right) \delta 174.4,140.7,139.6,114.1,112.6,101.6,81.0,70.6,62.7,59.7$, $48.8,47.0,37.3,35.7,33.0,32.9,28.9,26.1,22.0,18.4,-4.4,-4.5$; HRMS calcd for $\mathrm{C}_{24} \mathrm{H}_{45} \mathrm{O}_{5} \mathrm{NNaSi}[\mathrm{M}+\mathrm{Na}]: \quad 478.2959$; found: 478.2954 .

S. Kamijo, S. Yokosaka, M. Inoue, Tetrahedron 2012, 68, 5290.

\section{Preparation of compound 15}
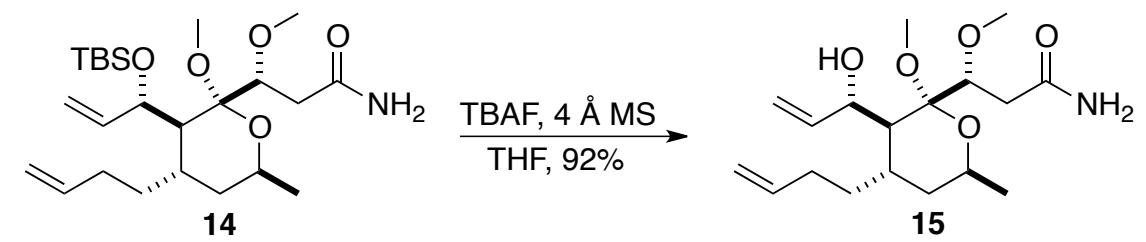
A suspension of TBS ether $14(0.90 \mathrm{~g}, 1.97 \mathrm{mmol})$ and activated $4 \AA \mathrm{MS}(0.20 \mathrm{~g})$ in anhydrous THF $(30 \mathrm{~mL})$ was stirred at room temperature for $10 \mathrm{~min}$, followed by the addition of TBAF $\left(1.0 \mathrm{M}\right.$ in THF) $(3.95 \mathrm{~mL}, 3.95 \mathrm{mmol})$. The resulting mixture was stirred at $55^{\circ} \mathrm{C}$ for $23 \mathrm{~h}$ and cooled to room temperature, quenched with water $(20 \mathrm{~mL})$, extracted with EtOAc $(40 \mathrm{~mL} \times 3)$, washed with water $(20 \mathrm{~mL} \times 3)$ and brine $(20 \mathrm{~mL} \times 3)$, dried $\left(\mathrm{MgSO}_{4}\right)$, filtered, concentrated, and purified by column chromatography (silica gel, petroleum ether:ethyl acetate $=1: 2$ ) to afford alcohol 15 as a white solid (0.62 g, 92\%). mp. $104-106^{\circ} \mathrm{C} ; \quad[\alpha]_{\mathrm{D}}{ }^{25}=-49.2\left(c 1.01, \mathrm{CHCl}_{3}\right) ; \quad$ IR (film): $3367,1668 \mathrm{~cm}^{-1} ; \quad{ }^{1} \mathrm{H}$ NMR (400 $\left.\mathrm{MHz}, \mathrm{CDCl}_{3}\right) \delta 5.92-5.70(\mathrm{~m}, 3 \mathrm{H}), 5.56(\mathrm{br} \mathrm{s}, 1 \mathrm{H}), 5.21(\mathrm{~d}, J=16.8 \mathrm{~Hz}, 1 \mathrm{H}), 5.11(\mathrm{~d}, J=$ $10.0 \mathrm{~Hz}, 1 \mathrm{H}), 4.98$ (d, $J=17.2 \mathrm{~Hz}, 1 \mathrm{H}), 4.93(\mathrm{~d}, J=10.0 \mathrm{~Hz}, 1 \mathrm{H}), 4.74$ (d, $J=3.6 \mathrm{~Hz}, 1 \mathrm{H})$, 4.44-4.35 (m, 1H), 3.96 (dd, $J=10.4,2.4 \mathrm{~Hz}, 1 \mathrm{H}), 3.85-3.73(\mathrm{~m}, 1 \mathrm{H}), 3.58(\mathrm{~s}, 3 \mathrm{H}), 3.20$ (s, $3 \mathrm{H}), 2.71(\mathrm{dd}, J=15.2,10.4 \mathrm{~Hz}, 1 \mathrm{H}), 2.56(\mathrm{dd}, J=15.2,2.4 \mathrm{~Hz}, 1 \mathrm{H}), 2.06-1.96(\mathrm{~m}, 2 \mathrm{H})$, 1.90-1.82 (m, 1H), 1.79-1.68 (m, 1H), 1.65-1.51 (m, 2H), 1.46-1.34 (m, 1H), 1.23-1.15 (m, $1 \mathrm{H}), 1.19(\mathrm{~d}, J=6.4 \mathrm{~Hz}, 3 \mathrm{H}) ;{ }^{13} \mathrm{C} \mathrm{NMR}\left(100 \mathrm{MHz}, \mathrm{CDCl}_{3}\right) \delta 173.9,140.1,139.3,115.1$, $114.5,102.7,80.7,72.8,63.3,61.3,47.1,46.6,37.2,34.4,33.2,32.7,32.0,22.0 ; \quad$ HRMS calcd for $\mathrm{C}_{18} \mathrm{H}_{31} \mathrm{O}_{5} \mathrm{NNa}[\mathrm{M}+\mathrm{Na}]$ : 364.2094; found: 364.2091 .

H. C. Kolb, S. V. Ley, A. M. Z. Slawin, D. J. Williams, J. Chem. Soc. Perkin Trans. 11992 , 2735 .

\section{Preparation of compound 5}

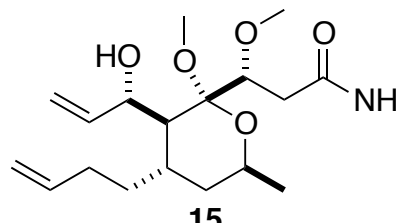

15

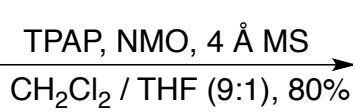

$\mathrm{CH}_{2} \mathrm{Cl}_{2} / \mathrm{THF}(9: 1), 80 \%$

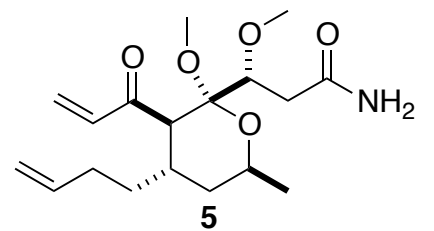

5

To a suspension of alcohol 15 (0.39 g, $1.14 \mathrm{mmol})$ and $4 \AA \mathrm{MS}(1.14 \mathrm{~g})$ in $\mathrm{CH}_{2} \mathrm{Cl}_{2}(27$ $\mathrm{mL})$ and $\mathrm{CH}_{3} \mathrm{CN}(3 \mathrm{~mL})$ was added $\mathrm{NMO}(0.21 \mathrm{~g}, 1.82 \mathrm{mmol})$ at $0{ }^{\circ} \mathrm{C}$. After stirred at $0{ }^{\circ} \mathrm{C}$ for $0.5 \mathrm{~h}$, TPAP $(0.040 \mathrm{~g}, 0.11 \mathrm{mmol})$ was added. Upon stirring at room temperature for $3 \mathrm{~h}$, the reaction mixture was concentrated, diluted with EtOAc, filtered through a plug of silica 
gel, concentrated, and purified by column chromatography (silica gel, petroleum ether:ethyl acetate $=1: 2)$ to afford ketone 5 as a colorless oil $(0.31 \mathrm{~g}, 80 \%) . \quad[\alpha]_{\mathrm{D}}{ }^{25}=-22.3(c 0.90$, $\left.\mathrm{CHCl}_{3}\right)$; IR (film): $3349,3198,1674,1612 \mathrm{~cm}^{-1} ;{ }^{1} \mathrm{H}$ NMR $\left(400 \mathrm{MHz}, \mathrm{CDCl}_{3}\right) \delta 6.58(\mathrm{dd}$, $J=17.2,10.4 \mathrm{~Hz}, 1 \mathrm{H}), 6.12$ (d, $J=17.2 \mathrm{~Hz}, 1 \mathrm{H}), 5.90$ (br s, 1H), 5.82-5.69 (m, 1H), 5.59 (d, $J=10.4 \mathrm{~Hz}, 1 \mathrm{H}), 5.64-5.52(\mathrm{~m}, 1 \mathrm{H}), 4.99$ (d, $J=17.2 \mathrm{~Hz}, 1 \mathrm{H}), 4.94(\mathrm{~d}, J=10.0 \mathrm{~Hz}, 1 \mathrm{H})$, 3.98-3.86 (m, 1H), $3.75(\mathrm{dd}, J=8.0,3.6 \mathrm{~Hz}, 1 \mathrm{H}), 3.26(\mathrm{~s}, 3 \mathrm{H}), 3.21(\mathrm{~s}, 3 \mathrm{H}), 2.85(\mathrm{~d}, J=2.8$ Hz, 1H), 2.69 (dd, $J=15.2,3.6 \mathrm{~Hz}, 1 \mathrm{H}), 2.30(\mathrm{dd}, J=15.2,8.0 \mathrm{~Hz}, 1 \mathrm{H}), 2.12-2.00(\mathrm{~m}, 2 \mathrm{H})$, $1.89-1.75(\mathrm{~m}, 2 \mathrm{H}), 1.74-1.62(\mathrm{~m}, 1 \mathrm{H}), 1.62-1.50(\mathrm{~m}, 1 \mathrm{H}), 1.40-1.31(\mathrm{~m}, 1 \mathrm{H}), 1.23(\mathrm{~d}, J=6.4$ $\mathrm{Hz}, 3 \mathrm{H}) ;{ }^{13} \mathrm{C} \mathrm{NMR}\left(100 \mathrm{MHz}, \mathrm{CDCl}_{3}\right) \delta 198.5,174.2,138.5,137.2,125.6,115.1,101.8$, $80.5,63.9,58.6,51.1,49.0,37.1,34.3,32.4,32.3,32.1,21.8$; HRMS calcd for $\mathrm{C}_{18} \mathrm{H}_{29} \mathrm{O}_{5} \mathrm{NNa}[\mathrm{M}+\mathrm{Na}]: 362.1938$; found: 362.1935 .

1) W. P. Griffith, S. V. Ley, G. P. Whitcombe, A. D. White, J. Chem. Soc., Chem. Commun. 1987, 1625.

2) M. T. Crimmins, A.-M. R. Dechert, Org. Lett. 2012, 14, 2366.

\section{Preparation of compound 4}

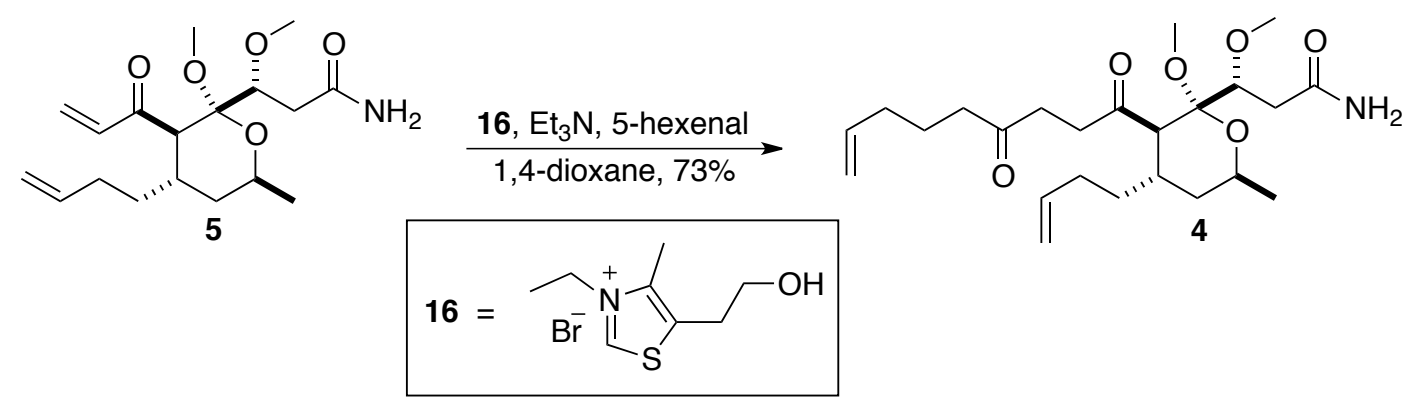

To a mixture of 3-ethyl-5-(2-hydroxyethyl)-4-methylthiazolium bromide (0.30 g, 1.18 $\mathrm{mmol})$, ketone 5 (0.31 g, $0.91 \mathrm{mmol})$, and 5-hexenal (0.36 g, $3.66 \mathrm{mmol})$ in 1,4-dioxane (30 $\mathrm{mL})$ was added $\mathrm{Et}_{3} \mathrm{~N}(0.55 \mathrm{~g}, 5.46 \mathrm{mmol})$ at room temperature under $\mathrm{N}_{2}$. The reaction mixture was stirred at $78{ }^{\circ} \mathrm{C}$ (bath temperature) for $18 \mathrm{~h}$ and cooled to room temperature, quenched with water $(30 \mathrm{~mL})$, extracted with EtOAc $(30 \mathrm{~mL} \times 3)$, washed with water $(10 \mathrm{~mL}$ $\times 3)$ and brine $(10 \mathrm{~mL} \times 3)$, dried $\left(\mathrm{MgSO}_{4}\right)$, filtered, concentrated, and purified by column chromatography (silica gel, petroleum ether:ethyl acetate $=1: 1$ to 1:2) to afford compound 4 
as a colorless oil $(0.29 \mathrm{~g}, 73 \%) . \quad[\alpha]_{\mathrm{D}}^{25}=-8.6\left(c 0.93, \mathrm{CHCl}_{3}\right) ; \quad$ IR (film): 3350, 3194, 1707, 1684, $1640 \mathrm{~cm}^{-1} ; \quad{ }^{1} \mathrm{H}$ NMR (400 MHz, $\left.\mathrm{CDCl}_{3}\right) \delta 5.92($ br s, $1 \mathrm{H}), 5.83-5.68(\mathrm{~m}, 2 \mathrm{H})$, 5.49 (br s, 1H), 5.06-4.90 (m, 4H), 3.94-3.82 (m, 1H), 3.69 (dd, J = 7.6, 4.0 Hz, 1H), 3.25 (s, $3 \mathrm{H}), 3.19$ (s, 3H), 2.90-2.76 (m, 2H), 2.74-2.52 (m, 4H), 2.47 (t, $J=7.2 \mathrm{~Hz}, 2 \mathrm{H}), 2.32$ (dd, $J=$ 14.8, $7.6 \mathrm{~Hz}, 1 \mathrm{H}), 2.13-1.98(\mathrm{~m}, 4 \mathrm{H}), 1.97-1.88(\mathrm{~m}, 1 \mathrm{H}), 1.87-1.76(\mathrm{~m}, 1 \mathrm{H}), 1.72-1.57(\mathrm{~m}$, $3 \mathrm{H}), 1.56-1.44(\mathrm{~m}, 1 \mathrm{H}), 1.38-1.30(\mathrm{~m}, 1 \mathrm{H}), 1.20(\mathrm{~d}, J=6.0 \mathrm{~Hz}, 3 \mathrm{H}) ;{ }^{13} \mathrm{C}$ NMR $(100 \mathrm{MHz}$, $\left.\mathrm{CDCl}_{3}\right) \delta 210.1,207.1$. 174.2, 138.6, 138.2, 115.4, 115.0, 101.7, 80.5, 64.2, 58.6, 52.0, 48.6, $42.3,37.5,36.8,36.4,34.3,33.3,32.6,32.1,31.8,23.0,21.7$; HRMS calcd for $\mathrm{C}_{24} \mathrm{H}_{39} \mathrm{O}_{6} \mathrm{NNa}[\mathrm{M}+\mathrm{Na}]: 460.2670 ;$ found: 460.2665 .

1) H. Stetter, Angew. Chem. Int. Ed. Engl. 1976, 15, 639.

2) P. E. Harrington, M. A. Tius, J. Am. Chem. Soc. 2001, 123, 8509.

3) A. Hall, S. Atkinson, S. H. Brown, I. P. Chessell, A. Chowdhury, G. M. P. Giblin, P. Goldsmith, M. P. Healy, K. S. Jandu, M. R. Johnson, A. D. Michel, A. Naylor, J. A. Sweeting, Bioorg. Med. Chem. Lett. 2007, 17, 1200.

\section{Preparation of compound 17}
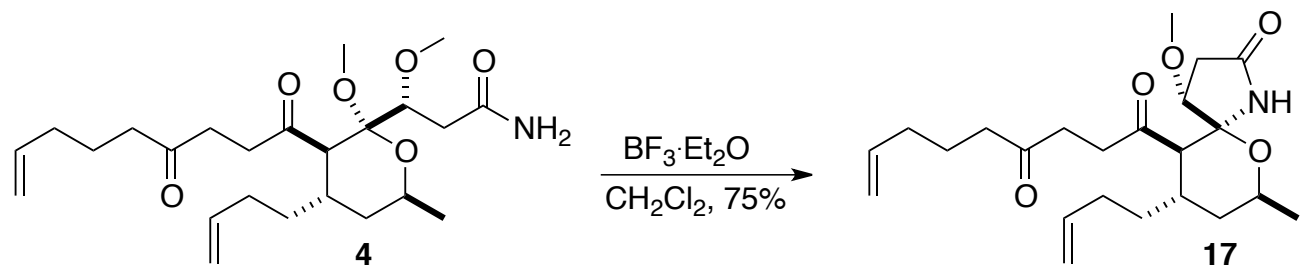

To a stirred solution of amide $4(0.29 \mathrm{~g}, 0.66 \mathrm{~mol})$ in $\mathrm{CH}_{2} \mathrm{Cl}_{2}(40 \mathrm{~mL})$ was added $\mathrm{BF}_{3} \cdot \mathrm{OEt}_{2}(0.28 \mathrm{~g}, 2.00 \mathrm{mmol})$. Upon stirring at room temperature for $15 \mathrm{~h}$, the reaction mixture was quenched with $\mathrm{Et}_{3} \mathrm{~N}(1.0 \mathrm{~mL})$, concentrated, and purified by column chromatography ( silica gel, petroleum ether:ethyl acetate $=2: 1$ ) to afford lactam $\mathbf{1 7}$ as a colorless oil $(0.20 \mathrm{~g}, 75 \%) . \quad[\alpha]_{\mathrm{D}}{ }^{25}=-34.0\left(c 0.80, \mathrm{CHCl}_{3}\right) ; \quad$ IR (film): $3233,1707,1640$ $\mathrm{cm}^{-1}$; ${ }^{1} \mathrm{H}$ NMR $\left(400 \mathrm{MHz}, \mathrm{CDCl}_{3}\right) \delta 6.84(\mathrm{~s}, 1 \mathrm{H}), 5.88-5.68(\mathrm{~m}, 2 \mathrm{H}), 5.14-4.90(\mathrm{~m}, 4 \mathrm{H})$, 3.80-3.67 (m, 2H), $3.10(\mathrm{~s}, 3 \mathrm{H}), 3.02-2.90(\mathrm{~m}, 1 \mathrm{H}), 2.84-2.71(\mathrm{~m}, 2 \mathrm{H}), 2.65-2.38(\mathrm{~m}, 5 \mathrm{H})$, 2.26-2.14 (m, 3H), 2.13-1.98 (m, 3H), 1.76-1.58 (m, 4H), 1.57-1.45 (m, 1H), 1.38-1.30 (m, $1 \mathrm{H}), 1.13(\mathrm{~d}, J=6.0 \mathrm{~Hz}, 3 \mathrm{H}) ;{ }^{13} \mathrm{C} \mathrm{NMR}\left(100 \mathrm{MHz}, \mathrm{CDCl}_{3}\right) \delta 209.6,205.9,177.5,138.2$, 
$137.5,116.0,115.4,91.7,82.8,62.5,56.3,50.3,42.3,35.7,33.8,33.3,32.3,31.9,31.7,23.0$, 21.8; HRMS calcd for $\mathrm{C}_{23} \mathrm{H}_{35} \mathrm{O}_{5} \mathrm{NNa}[\mathrm{M}+\mathrm{Na}]$ : 428.2407; found: 428.2404.

K. C. Nicolaou, P. M. Pihko, N. Diedrichs, N. Zou, F. Bernal, Angew. Chem. Int. Ed. 2001, $40,1262$.

\section{Preparation of compound 18}
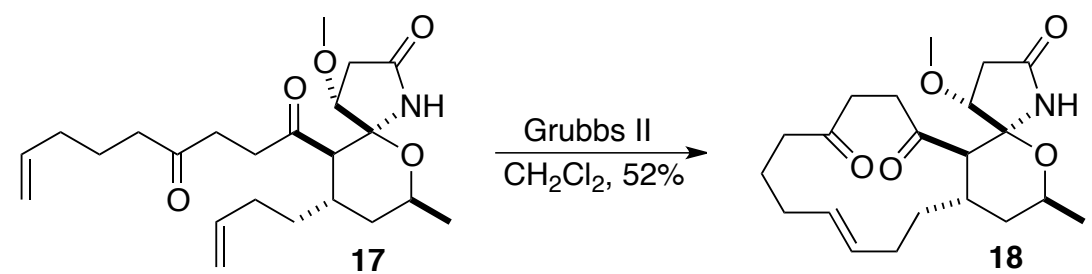

18

To a solution of Grubbs' II catalyst $(0.0419 \mathrm{~g}, 0.049 \mathrm{mmol})$ in $\mathrm{CH}_{2} \mathrm{Cl}_{2}(400 \mathrm{~mL})$ was added to a solution of compound $17(0.20 \mathrm{~g}, 0.49 \mathrm{mmol})$ in $\mathrm{CH}_{2} \mathrm{Cl}_{2}(90 \mathrm{~mL})$ under $\mathrm{N}_{2}$. The reaction mixture was heated at reflux for $36 \mathrm{~h}$, cooled to room temperature, quenched with ethyl vinyl ether $(5.0 \mathrm{~mL})$, stirred at room temperature for $0.5 \mathrm{~h}$, concentrated, and purified by column chromatography ( compound 18 as a white solid $(0.0965 \mathrm{~g}, 52 \%)$. mp. $192-193{ }^{\circ} \mathrm{C} ; \quad[\alpha]_{\mathrm{D}}{ }^{25}=-90.4(c 0.59$, $\mathrm{CHCl}_{3}$ ); IR (film): 3247, $1706 \mathrm{~cm}^{-1} ;{ }^{1} \mathrm{H} \mathrm{NMR}\left(500 \mathrm{MHz}, \mathrm{CDCl}_{3}\right) \delta 6.69$ (br s, $\left.1 \mathrm{H}\right)$, 5.28-5.20 (m, 2H), 3.77-3.66 (m, 2H), $3.12(\mathrm{~s}, 3 \mathrm{H}), 2.94-2.83(\mathrm{~m}, 1 \mathrm{H}), 2.66-2.46(\mathrm{~m}, 5 \mathrm{H})$, 2.45-2.32 (m, 2H), 2.28-2.12 (m, 3H), 1.96-1.84 (m, 3H), 1.83-1.73 (m, 2H), 1.70-1.52 (m, 2H), 1.40-1.25 (m, 2H), $1.16(\mathrm{~d}, J=6.0 \mathrm{~Hz}, 3 \mathrm{H}) ;{ }^{13} \mathrm{C} \mathrm{NMR}\left(125 \mathrm{MHz}, \mathrm{CDCl}_{3}\right) \delta 209.5$, 205.3, 177.2, 133.6, 129.9, 91.6, 82.8, 62.3, 56.2, 51.0, 41.2, 38.8, 38.0, 34.0, 33.7, 33.3, 30.0, 29.7, 27.7, 21.8, 21.2; HRMS calcd for $\mathrm{C}_{21} \mathrm{H}_{31} \mathrm{O}_{5} \mathrm{NNa}[\mathrm{M}+\mathrm{Na}]$ : 400.2094; found: 400.2092.

1) M. Scholl, S. Ding, C. W. Lee, R. H. Grubbs, Org. Lett. 1999, 1, 953.

2) P. E. Harrington, M. A. Tius, J. Am. Chem. Soc. 2001, 123, 8509. 


\section{Preparation of compound 19}
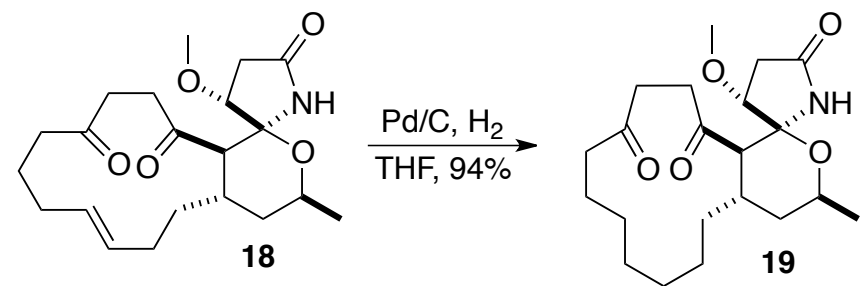

A mixture of compound $18(0.0632 \mathrm{~g}, 0.17 \mathrm{mmol})$ and $10 \% \mathrm{Pd} / \mathrm{C}(0.0357 \mathrm{~g}, 0.034 \mathrm{mmol})$ in THF (15 mL) under $\mathrm{H}_{2}$ atmosphere (with a balloon) was stirred at room temperature for 12 h, concentrated, and purified by column chromatography (silica gel, petroleum ether:ethyl acetate $=1: 2)$ to afford compound 19 as a white solid $(0.0601 \mathrm{~g}, 94 \%)$. mp. 192-193 ${ }^{\circ} \mathrm{C}$; $[\alpha]_{\mathrm{D}}{ }^{25}=-40.3\left(c 1.04, \mathrm{CHCl}_{3}\right) ; \quad$ IR (film): $3189,1704,1645 \mathrm{~cm}^{-1} ; \quad{ }^{1} \mathrm{H}$ NMR $(500 \mathrm{MHz}$, $\left.\mathrm{CDCl}_{3}\right) \delta 7.01($ br s, $1 \mathrm{H}), 3.85-3.75(\mathrm{~m}, 1 \mathrm{H}), 3.73-3.66(\mathrm{~m}, 1 \mathrm{H}), 3.11(\mathrm{~s}, 3 \mathrm{H}), 2.95-2.85(\mathrm{~m}$, $1 \mathrm{H}), 2.78-2.69(\mathrm{~m}, 2 \mathrm{H}), 2.69-2.57(\mathrm{~m}, 3 \mathrm{H}), 2.54-2.42(\mathrm{~m}, 2 \mathrm{H}), 2.22(\mathrm{~d}, J=17.0 \mathrm{~Hz}, 1 \mathrm{H})$, $1.87-1.71(\mathrm{~m}, 2 \mathrm{H}), 1.69-1.57(\mathrm{~m}, 2 \mathrm{H}), 1.50-1.29(\mathrm{~m}, 8 \mathrm{H}), 1.28-1.11(\mathrm{~m}, 3 \mathrm{H}), 1.18(\mathrm{~d}, J=6.0$

$\mathrm{Hz}, 3 \mathrm{H}) ; \quad{ }^{13} \mathrm{C} \mathrm{NMR}\left(125 \mathrm{MHz}, \mathrm{CDCl}_{3}\right) \delta 210.8,207.2,177.4,92.1,82.8,63.0,55.9,52.5$, $41.4,39.6,37.4,34.3,31.3,31.0,29.7,27.1,24.9,24.8,23.7,21.8,21.1 ; \quad$ HRMS calcd for $\mathrm{C}_{21} \mathrm{H}_{33} \mathrm{O}_{5} \mathrm{NNa}[\mathrm{M}+\mathrm{Na}]:$ 402.2251; found: 402.2249 .

P. E. Harrington, M. A. Tius, J. Am. Chem. Soc. 2001, 123, 8509.

\section{Preparation of compound 1}
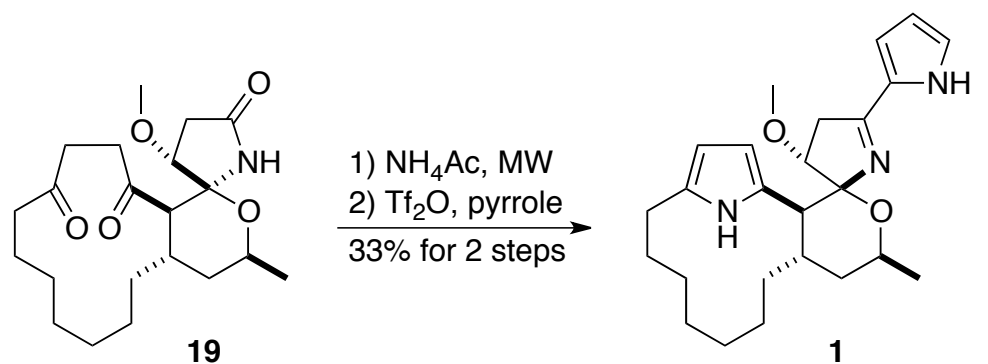

A mixture of compound $19(0.0302 \mathrm{~g}, 0.080 \mathrm{mmol}), \mathrm{NH}_{4} \mathrm{Ac}(0.246 \mathrm{~g}, 3.19 \mathrm{mmol})$, and anhydrous $\mathrm{MeOH}(5.0 \mathrm{~mL})$ in a sealed vial was heated at $140{ }^{\circ} \mathrm{C}$ under microwave for $25 \mathrm{~min}$ and then cooled to room temperature. ${ }^{1} \quad$ Another portion of $\mathrm{NH}_{4} \mathrm{Ac}(0.246 \mathrm{~g}, 3.19 \mathrm{mmol})$ was added. Upon heating at $140{ }^{\circ} \mathrm{C}$ under microwave for another $25 \mathrm{~min}$, the reaction mixture was cooled to room temperature, quenched with water $(30 \mathrm{~mL})$, extracted with $\mathrm{CH}_{2} \mathrm{Cl}_{2}(20$ 
$\mathrm{mL} \times 3)$, washed with water $(10 \mathrm{~mL} \times 3)$ and brine $(10 \mathrm{~mL} \times 3)$, dried $\left(\mathrm{Na}_{2} \mathrm{SO}_{4}\right)$, filtered, concentrated to give a residue, which was dissolved in freshly distilled pyrrole $(3.0 \mathrm{~mL})$. To the solution, $\mathrm{Tf}_{2} \mathrm{O}(0.0449 \mathrm{~g}, 0.16 \mathrm{mmol})$ was added dropwise at $0{ }^{\circ} \mathrm{C}$ under $\mathrm{N}_{2} .{ }^{2}$ Upon stirring at room temperature for $3 \mathrm{~h}$, the reaction mixture was quenched with saturated aqueous $\mathrm{NaHCO}_{3}$ solution $(10 \mathrm{~mL})$, extracted with $\mathrm{CH}_{2} \mathrm{Cl}_{2}(20 \mathrm{~mL} \times 3)$, washed with water $(10 \mathrm{~mL} \times 3)$ and brine $(10 \mathrm{~mL} \times 3)$, dried $\left(\mathrm{Na}_{2} \mathrm{SO}_{4}\right)$, filtered, concentrated, and purified by column chromatography (silica gel, hexane:ether $=5: 1$ to $2: 1$ ) to afford compound $\mathbf{1}$ as a white solid $(0.0108 \mathrm{~g}, 33 \%$ for 2 steps $)$. mp. $120-121{ }^{\circ} \mathrm{C} ; \quad[\alpha]_{\mathrm{D}}{ }^{25}=62.6(c 0.16, \mathrm{MeOH})$, $[\alpha]_{\mathrm{D}}^{25}=190.0$ (c 0.05, Acetone); IR (film): 3397, 3367, 2920, 2848, 1645, 1470, 1421 $\mathrm{cm}^{-1} ; \quad{ }^{1} \mathrm{H}$ NMR $\left(500 \mathrm{MHz}\right.$, Acetone- $\left.d_{6}\right) \delta 8.71(\mathrm{br} \mathrm{s}, 1 \mathrm{H}), 6.99(\mathrm{~s}, 1 \mathrm{H}), 6.50(\mathrm{~d}, J=3.5 \mathrm{~Hz}$, 1H), $6.15(\mathrm{~s}, 1 \mathrm{H}), 5.77(\mathrm{~s}, 1 \mathrm{H}), 5.56(\mathrm{~s}, 1 \mathrm{H}), 4.27-4.18(\mathrm{~m}, 1 \mathrm{H}), 3.92(\mathrm{t}, J=7.5 \mathrm{~Hz}, 1 \mathrm{H}), 3.42$ (s, 3H), 2.80 (dd, $J=16.0,7.5 \mathrm{~Hz}, 1 \mathrm{H}), 2.73$ (d, $J=12.0 \mathrm{~Hz}, 1 \mathrm{H}), 2.71$ (dd, $J=15.5,8.0 \mathrm{~Hz}$, $1 \mathrm{H}), 2.56-2.42(\mathrm{~m}, 2 \mathrm{H}), 2.38-2.28(\mathrm{~m}, 1 \mathrm{H}), 1.83-1.71(\mathrm{~m}, 2 \mathrm{H}), 1.67-1.54(\mathrm{~m}, 2 \mathrm{H}), 1.52-1.34$ $(\mathrm{m}, 4 \mathrm{H}), 1.34(\mathrm{~d}, J=6.5 \mathrm{~Hz}, 3 \mathrm{H}), 1.22-1.11(\mathrm{~m}, 1 \mathrm{H}), 1.10-1.01(\mathrm{~m}, 1 \mathrm{H}), 0.99-0.91(\mathrm{~m}, 1 \mathrm{H})$, 0.85-0.73 (m, 2H), 0.65-0.52 (m, 1H); ${ }^{13} \mathrm{C}$ NMR (125 MHz, Acetone- $\left.d_{6}\right) \delta 165.2,132.8$, $130.4,129.3,123.0,114.0,110.2$, 105.8, 101.6, 83.7, 69.7, 58.5, 49.4, 39.9, 38.2, 32.8, 28.9, 27.6, 25.8, 25.7, 25.4, 23.5; HRMS calcd for $\mathrm{C}_{25} \mathrm{H}_{36} \mathrm{O}_{2} \mathrm{~N}_{3}[\mathrm{M}+\mathrm{H}]$ : 410.2802; found: 410.2797 .

1) a) L. Knorr, Ber. Dtsch. Chem. Ges. 1885, 18, 299. b) C. Paal, Ber. Dtsch. Chem. Ges. 1885, 18, 367. c) L. N. Aldrich, C. B. Berry, B. S. Bates, L. C. Konkol, M. So, C. W. Lindsley, Eur. J. Org. Chem. 2013, 2013, 4215. d) S. Khaghaninejad, M. M. Heravi, Adv. Heterocycl. Chem. 2014, 111, 95.

2) a) A. G. Martínez, R. M. Alvarez, J. O. Barcina, S. M. Cerero, E. T. Vilar, A. G. Fraile, M. Hanack, L. R. Subramanian, J. Chem. Soc., Chem. Commun. 1990, 1571.

b) G. Li, X. Zhang, Q. Li, P. Feng, Y. Shi, Org. Biomol. Chem. 2013, 11, 2936. 
Table 1. Comparison of ${ }^{1} \mathrm{H}$ NMR data in Acetone- $d_{6}$

\begin{tabular}{|l|l|}
\hline Synthetic compound & Natural compound $^{\mathrm{a}}$ \\
\hline & $10.93(\mathrm{br} \mathrm{s}, 1 \mathrm{H})$ \\
\hline $8.71(\mathrm{br} \mathrm{s}, 1 \mathrm{H})$ & $8.23(\mathrm{br} \mathrm{s}, 1 \mathrm{H})$ \\
\hline $6.99(\mathrm{~s}, 1 \mathrm{H})$ & $6.97(\mathrm{dd}, J=2.5,1.5 \mathrm{~Hz}, 1 \mathrm{H})$ \\
\hline $6.50(\mathrm{~d}, J=3.5 \mathrm{~Hz}, 1 \mathrm{H})$ & $6.37(\mathrm{dd}, J=3.5,1.5 \mathrm{~Hz}, 1 \mathrm{H})$ \\
\hline $6.15(\mathrm{~s}, 1 \mathrm{H})$ & $6.11(\mathrm{dd}, J=3.5,2.5 \mathrm{~Hz}, 1 \mathrm{H})$ \\
\hline $5.77(\mathrm{~s}, 1 \mathrm{H})$ & $5.68(\mathrm{~d}, J=3.0 \mathrm{~Hz}, 1 \mathrm{H})$, \\
\hline $5.56(\mathrm{~s}, 1 \mathrm{H})$ & $5.44(\mathrm{~d}, J=3.0 \mathrm{~Hz}, 1 \mathrm{H})$ \\
\hline $4.27-4.18(\mathrm{~m}, 1 \mathrm{H})$ & $4.23(\mathrm{~m}, 1 \mathrm{H})$ \\
\hline $3.92(\mathrm{t}, J=7.5 \mathrm{~Hz}, 1 \mathrm{H})$ & $3.85(\mathrm{t}, J=8.5 \mathrm{~Hz}, 1 \mathrm{H})$ \\
\hline $3.42(\mathrm{~s}, 3 \mathrm{H})$ & $3.40(\mathrm{~s}, 3 \mathrm{H})$ \\
\hline $2.80(\mathrm{dd}, J=16.0,7.5 \mathrm{~Hz}, 1 \mathrm{H})$ & $2.91(\mathrm{~d}, J=12.0 \mathrm{~Hz}, 1 \mathrm{H})$ \\
\hline $2.73(\mathrm{~d}, J=12.0 \mathrm{~Hz}, 1 \mathrm{H})$ & $2.88(\mathrm{dd}, J=16.0,8.5 \mathrm{~Hz}, 1 \mathrm{H})$ \\
\hline $2.71(\mathrm{dd}, J=15.5,8.0 \mathrm{~Hz}, 1 \mathrm{H})$ & $2.32(\mathrm{~m}, 1 \mathrm{H})$ \\
\hline $2.56-2.42(\mathrm{~m}, 2 \mathrm{H})$ & $2.23(\mathrm{~m}, 2 \mathrm{H})$, \\
\hline $2.38-2.28(\mathrm{~m}, 1 \mathrm{H})$ & $1.88(\mathrm{dd}, J=16.0,8.5 \mathrm{~Hz}, 1 \mathrm{H})$ \\
\hline $1.83-1.71(\mathrm{~m}, 2 \mathrm{H})$ & $1.77(\mathrm{~m}, 1 \mathrm{H})$ \\
\hline $1.67-1.54(\mathrm{~m}, 2 \mathrm{H})$ & $1.64(\mathrm{~m}, 1 \mathrm{H})$ \\
\hline $1.52-1.34(\mathrm{~m}, 4 \mathrm{H})$ & $1.61(\mathrm{td}, J=12.0,6.5 \mathrm{~Hz}, 1 \mathrm{H})$ \\
\hline $1.34(\mathrm{~d}, J=6.5 \mathrm{~Hz}, 3 \mathrm{H})$ & $1.52(\mathrm{~m}, 1 \mathrm{H})$ \\
\hline $1.22-1.11(\mathrm{~m}, 1 \mathrm{H})$ & $1.51(\mathrm{~d}, J=6.5 \mathrm{~Hz}, 3 \mathrm{H})$ \\
\hline $1.10-1.01(\mathrm{~m}, 1 \mathrm{H})$ & $1.37(\mathrm{~m}, 1 \mathrm{H})$ \\
\hline $0.99-0.91(\mathrm{~m}, 1 \mathrm{H})$ & $1.35(\mathrm{~m}, 1 \mathrm{H})$ \\
\hline $0.85-0.73(\mathrm{~m}, 2 \mathrm{H})$ & $1.34(\mathrm{~m}, 1 \mathrm{H})$ \\
\hline $0.65-0.52(\mathrm{~m}, 1 \mathrm{H})$ & $1.28(\mathrm{~m}, 1 \mathrm{H})$ \\
\hline & $1.18(\mathrm{~m}, 1 \mathrm{H})$ \\
\hline & $1.02(\mathrm{~m}, 1 \mathrm{H})$ \\
\hline & $0.88(\mathrm{~m}, 2 \mathrm{H})$ \\
\hline & $0.69(\mathrm{~m}, 1 \mathrm{H})$ \\
\hline & $0.52(\mathrm{~m}, 1 \mathrm{H})$ \\
\hline & \\
\hline
\end{tabular}

${ }^{a}$ The NMR data of the natural compound is taken from Boonlarppradab, C.; Kauffman, C. A.; Jensen, P. R.; Fenical, W. Org. Lett. 2008, 10, 5505. 
Table 2. Comparison of ${ }^{13} \mathrm{C}$ NMR data in Acetone- $d_{6}$

\begin{tabular}{|c|c|}
\hline Synthetic compound & Natural compound $^{\mathrm{a}}$ \\
\hline 165.2 & 164.4 \\
\hline 132.8 & 131.6 \\
\hline 130.4 & 129.9 \\
\hline 129.3 & 129.1 \\
\hline 123.0 & 122.5 \\
\hline 114.0 & 113.5 \\
\hline \multirow[t]{2}{*}{110.2} & 110.1 \\
\hline & 109.9 \\
\hline 105.8 & 106.1 \\
\hline 101.6 & 104.7 \\
\hline 83.7 & 89.9 \\
\hline 69.7 & 70.4 \\
\hline 58.5 & 58.5 \\
\hline 49.4 & 45.9 \\
\hline 39.9 & 39.6 \\
\hline 38.2 & 39.0 \\
\hline \multirow[t]{2}{*}{32.8} & 32.0 \\
\hline & 29.6 \\
\hline 28.9 & 28.6 \\
\hline 27.6 & 28.0 \\
\hline 25.8 & 25.7 \\
\hline 25.7 & 25.5 \\
\hline \multirow[t]{2}{*}{25.4} & 25.5 \\
\hline & 25.4 \\
\hline 23.5 & 22.8 \\
\hline
\end{tabular}

${ }^{\mathrm{a}}$ The NMR data of the natural compound is taken from Boonlarppradab, C.; Kauffman, C. A.; Jensen, P. R.; Fenical, W. Org. Lett. 2008, 10, 5505. 
The X-ray structure of compound 12
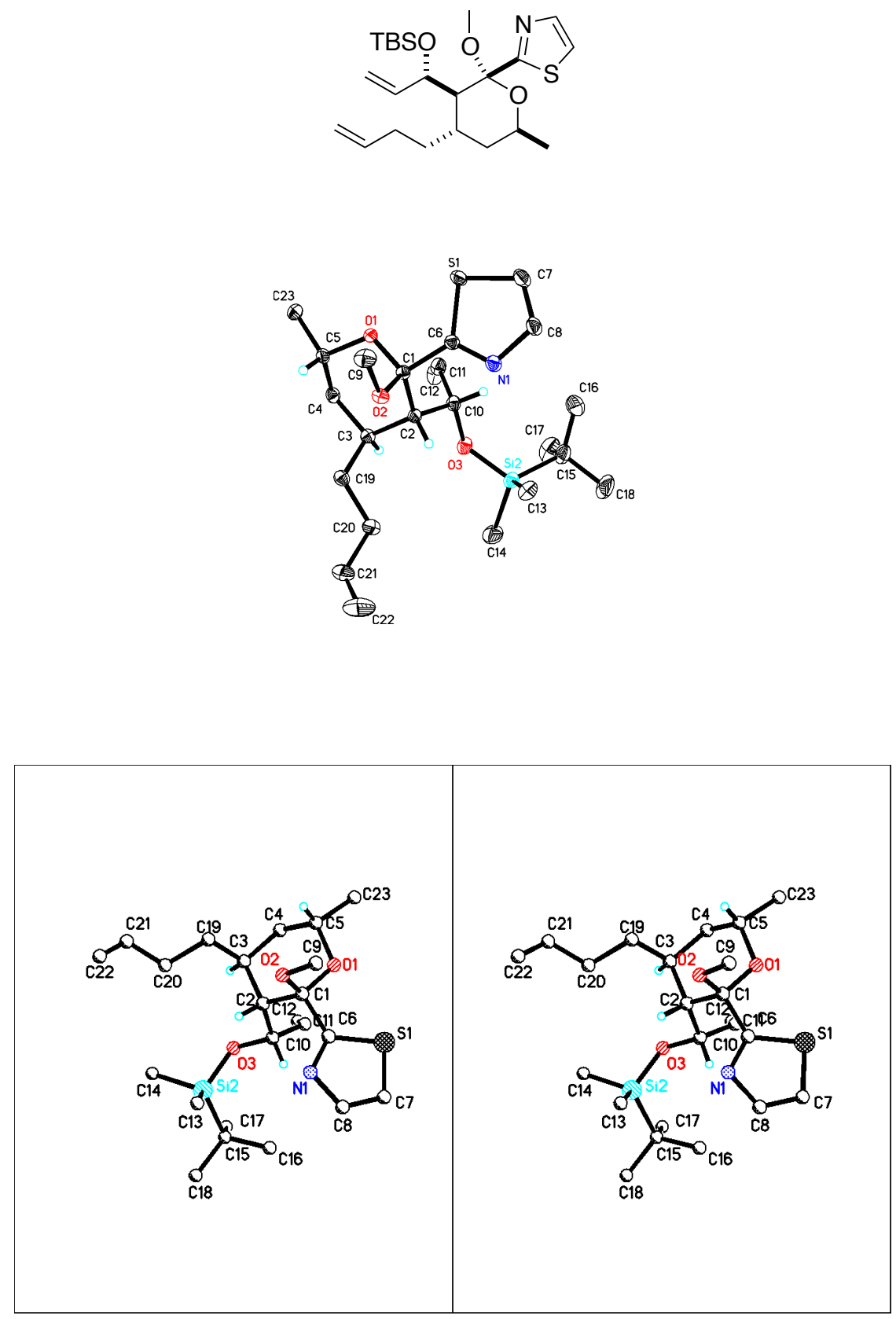
Table 1. Crystal data and structure refinement for $\mathbf{1 2}$.

Identification code

Empirical formula

Formula weight

Temperature

Wavelength

Crystal system

Space group

Unit cell dimensions

Volume

Z

Density (calculated)

Absorption coefficient

$\mathrm{F}(000)$

Crystal size

Theta range for data collection

Index ranges

Reflections collected

Independent reflections

Completeness to theta $=26.000^{\circ}$

Absorption correction

Max. and min. transmission

Refinement method

Data / restraints / parameters

Goodness-of-fit on $\mathrm{F}^{2}$

Final $\mathrm{R}$ indices $[\mathrm{I}>2 \operatorname{sigma}(\mathrm{I})]$

$\mathrm{R}$ indices (all data)

Absolute structure parameter

Extinction coefficient

Largest diff. peak and hole
12

$\mathrm{C}_{23} \mathrm{H}_{39} \mathrm{~N} \mathrm{O}_{3} \mathrm{~S} \mathrm{Si}$

437.70

$173.1500 \mathrm{~K}$

$0.71073 \AA$

Orthorhombic

P 212121

$\mathrm{a}=11.2183(14) \AA \quad \mathrm{a}=90^{\circ}$.

$\mathrm{b}=17.958(2) \AA \quad \mathrm{b}=90^{\circ}$.

$\mathrm{c}=25.733(2) \AA \quad \mathrm{g}=90^{\circ}$.

5184.1(10) $\AA^{3}$

8

$1.122 \mathrm{Mg} / \mathrm{m}^{3}$

$0.193 \mathrm{~mm}^{-1}$

1904

$0.52 \times 0.19 \times 0.14 \mathrm{~mm}^{3}$

1.947 to $27.477^{\circ}$.

$-14<=\mathrm{h}<=14,-23<=\mathrm{k}<=23,-33<=\mathrm{k}<=33$

35540

$11719[\mathrm{R}(\mathrm{int})=0.0378]$

$99.6 \%$

Semi-empirical from equivalents

1.0000 and 0.7954

Full-matrix least-squares on $\mathrm{F}^{2}$

11719 / 0 / 537

1.048

$\mathrm{R} 1=0.0444, \mathrm{wR} 2=0.0988$

$\mathrm{R} 1=0.0469, \mathrm{wR} 2=0.1010$

$0.02(2)$

$\mathrm{n} / \mathrm{a}$

0.380 and -0.216 e. $\AA^{-3}$ 
Table 2. Atomic coordinates (x 104) and equivalent isotropic displacement parameters $\left(\AA^{2} \mathrm{x}\right.$ $10^{3}$ ) for 12 . $U(\mathrm{eq})$ is defined as one third of the trace of the orthogonalized Uij tensor.

\begin{tabular}{|c|c|c|c|c|}
\hline & $\mathrm{x}$ & $\mathrm{y}$ & $\mathrm{z}$ & $\mathrm{U}(\mathrm{eq})$ \\
\hline $\mathrm{S} 1$ & $5549(1)$ & $6331(1)$ & $4373(1)$ & $37(1)$ \\
\hline $\mathrm{Si} 2$ & $8144(1)$ & $3652(1)$ & $5359(1)$ & $35(1)$ \\
\hline $\mathrm{O} 1$ & $7428(2)$ & $6846(1)$ & 4989(1) & $25(1)$ \\
\hline $\mathrm{O} 2$ & $8944(2)$ & $6423(1)$ & $4442(1)$ & $26(1)$ \\
\hline $\mathrm{O} 3$ & $7907(2)$ & $4495(1)$ & $5594(1)$ & $35(1)$ \\
\hline N1 & $7084(2)$ & $5375(1)$ & $4070(1)$ & $30(1)$ \\
\hline $\mathrm{C} 1$ & $7926(2)$ & $6222(1)$ & $4739(1)$ & $23(1)$ \\
\hline $\mathrm{C} 2$ & $8360(2)$ & $5616(1)$ & $5118(1)$ & $24(1)$ \\
\hline $\mathrm{C} 3$ & $9132(2)$ & $5940(1)$ & $5564(1)$ & $26(1)$ \\
\hline $\mathrm{C} 4$ & $8613(3)$ & $6676(1)$ & $5770(1)$ & $29(1)$ \\
\hline $\mathrm{C} 5$ & $8238(2)$ & $7213(1)$ & $5345(1)$ & $27(1)$ \\
\hline C6 & $6949(2)$ & $5940(1)$ & $4379(1)$ & $26(1)$ \\
\hline $\mathrm{C} 7$ & $5115(3)$ & $5681(2)$ & $3925(1)$ & $38(1)$ \\
\hline $\mathrm{C} 8$ & $6033(3)$ & $5230(2)$ & $3810(1)$ & $34(1)$ \\
\hline C9 & $8730(3)$ & $6978(2)$ & $4055(1)$ & $38(1)$ \\
\hline $\mathrm{C} 10$ & $7356(2)$ & $5102(1)$ & $5329(1)$ & $29(1)$ \\
\hline $\mathrm{C} 11$ & $6474(3)$ & $5483(2)$ & $5680(1)$ & $37(1)$ \\
\hline $\mathrm{C} 12$ & $6250(4)$ & $5297(2)$ & $6163(1)$ & $54(1)$ \\
\hline $\mathrm{C} 13$ & $8660(3)$ & $3703(2)$ & $4669(1)$ & $44(1)$ \\
\hline $\mathrm{C} 14$ & $9318(4)$ & $3270(2)$ & $5794(2)$ & $58(1)$ \\
\hline $\mathrm{C} 15$ & $6741(3)$ & $3064(2)$ & $5397(2)$ & $49(1)$ \\
\hline C16 & $5731(4)$ & $3427(2)$ & $5091(2)$ & $69(1)$ \\
\hline $\mathrm{C} 17$ & $6352(5)$ & $2981(2)$ & $5963(2)$ & $75(1)$ \\
\hline C18 & $7007(4)$ & $2281(2)$ & $5174(2)$ & $73(1)$ \\
\hline C19 & $10453(3)$ & $6004(1)$ & $5421(1)$ & $29(1)$ \\
\hline $\mathrm{C} 20$ & $11088(3)$ & $5252(2)$ & $5382(1)$ & $39(1)$ \\
\hline $\mathrm{C} 21$ & $12397(3)$ & $5305(2)$ & $5312(1)$ & $46(1)$ \\
\hline $\mathrm{C} 22$ & $13022(4)$ & 4990(3) & $4950(2)$ & $72(1)$ \\
\hline $\mathrm{C} 23$ & $7558(3)$ & $7876(2)$ & $5552(1)$ & $34(1)$ \\
\hline S1A & $510(1)$ & $4585(1)$ & $3185(1)$ & $38(1)$ \\
\hline $\mathrm{Si} 2 \mathrm{~A}$ & $3040(1)$ & $7312(1)$ & $2324(1)$ & $29(1)$ \\
\hline O1A & $2448(2)$ & $4077(1)$ & $2577(1)$ & $30(1)$ \\
\hline $\mathrm{O} 2 \mathrm{~A}$ & $3896(2)$ & $4455(1)$ & $3173(1)$ & $28(1)$ \\
\hline $\mathrm{O} 3 \mathrm{~A}$ & $2979(2)$ & $6469(1)$ & 2071(1) & $31(1)$ \\
\hline N1A & $2071(2)$ & $5472(1)$ & $3558(1)$ & $29(1)$ \\
\hline $\mathrm{C} 1 \mathrm{~A}$ & $2921(2)$ & $4683(1)$ & $2859(1)$ & $26(1)$ \\
\hline $\mathrm{C} 2 \mathrm{~A}$ & $3405(2)$ & $5308(1)$ & $2505(1)$ & $26(1)$ \\
\hline C3A & $4244(2)$ & $5015(2)$ & $2076(1)$ & $31(1)$ \\
\hline $\mathrm{C} 4 \mathrm{~A}$ & $3763(3)$ & $4283(2)$ & 1841(1) & $34(1)$ \\
\hline
\end{tabular}




$\begin{array}{lrrrr}\text { C5A } & 3319(3) & 3726(1) & 2236(1) & 32(1) \\ \text { C6A } & 1919(2) & 4952(1) & 3208(1) & 26(1) \\ \text { C7A } & 92(3) & 5177(2) & 3673(1) & 35(1) \\ \text { C8A } & 1023(2) & 5595(2) & 3822(1) & 30(1) \\ \text { C9A } & 3608(3) & 3891(2) & 3549(1) & 37(1) \\ \text { C10A } & 2418(2) & 5826(1) & 2283(1) & 28(1) \\ \text { C11A } & 1623(3) & 5483(2) & 1878(1) & 35(1) \\ \text { C12A } & 1493(3) & 5734(2) & 1401(1) & 46(1) \\ \text { C13A } & 3986(3) & 7309(2) & 2918(1) & 48(1) \\ \text { C14A } & 3762(4) & 7866(2) & 1800(1) & 55(1) \\ \text { C15A } & 1527(3) & 7717(2) & 2485(1) & 34(1) \\ \text { C16A } & 977(3) & 7372(2) & 2974(1) & 53(1) \\ \text { C17A } & 664(3) & 7613(2) & 2032(1) & 49(1) \\ \text { C18A } & 1692(3) & 8554(2) & 2588(1) & 47(1) \\ \text { C19A } & 5551(3) & 4945(2) & 2243(1) & 34(1) \\ \text { C20A } & 6133(3) & 5689(2) & 2390(1) & 44(1) \\ \text { C21A } & 7418(3) & 5620(2) & 2546(2) & 57(1) \\ \text { C22A } & 8308(4) & 5972(3) & 2384(2) & 77(1) \\ \text { C23A } & 2681(3) & 3071(2) & 1992(1) & 47(1)\end{array}$


Table 3. Bond lengths $[\AA]$ and angles $\left[{ }^{\circ}\right]$ for $\mathbf{1 2}$.

\begin{tabular}{|c|c|}
\hline S1-C6 & $1.721(3)$ \\
\hline S1-C7 & $1.711(3)$ \\
\hline $\mathrm{Si2}-\mathrm{O} 3$ & $1.652(2)$ \\
\hline $\mathrm{Si} 2-\mathrm{C} 13$ & $1.869(3)$ \\
\hline $\mathrm{Si} 2-\mathrm{C} 14$ & $1.859(4)$ \\
\hline $\mathrm{Si} 2-\mathrm{C} 15$ & $1.898(4)$ \\
\hline $\mathrm{O} 1-\mathrm{C} 1$ & $1.408(3)$ \\
\hline $\mathrm{O} 1-\mathrm{C} 5$ & $1.450(3)$ \\
\hline $\mathrm{O} 2-\mathrm{C} 1$ & $1.421(3)$ \\
\hline O2-C9 & $1.430(3)$ \\
\hline $\mathrm{O} 3-\mathrm{C} 10$ & $1.426(3)$ \\
\hline N1-C6 & $1.299(3)$ \\
\hline $\mathrm{N} 1-\mathrm{C} 8$ & $1.380(4)$ \\
\hline $\mathrm{C} 1-\mathrm{C} 2$ & $1.540(3)$ \\
\hline C1-C6 & $1.522(3)$ \\
\hline $\mathrm{C} 2-\mathrm{H} 2$ & 0.9800 \\
\hline $\mathrm{C} 2-\mathrm{C} 3$ & $1.550(3)$ \\
\hline $\mathrm{C} 2-\mathrm{C} 10$ & $1.553(4)$ \\
\hline C3-H3 & 0.9800 \\
\hline C3-C4 & $1.538(3)$ \\
\hline C3-C19 & $1.531(4)$ \\
\hline C4-H4A & 0.9700 \\
\hline C4-H4B & 0.9700 \\
\hline C4-C5 & $1.516(4)$ \\
\hline C5-H5 & 0.9800 \\
\hline C5-C23 & $1.511(3)$ \\
\hline C7-H7 & 0.9300 \\
\hline $\mathrm{C} 7-\mathrm{C} 8$ & $1.343(4)$ \\
\hline C8-H8 & 0.9300 \\
\hline C9-H9A & 0.9600 \\
\hline C9-H9B & 0.9600 \\
\hline C9-H9C & 0.9600 \\
\hline C10-H10 & 0.9800 \\
\hline C10-C11 & $1.505(4)$ \\
\hline C11-H11 & 0.9300 \\
\hline C11-C12 & $1.309(4)$ \\
\hline C12-H12A & 0.9300 \\
\hline C12-H12B & 0.9300 \\
\hline C13-H13A & 0.9600 \\
\hline C13-H13B & 0.9600 \\
\hline C13-H13C & 0.9600 \\
\hline C14-H14A & 0.9600 \\
\hline
\end{tabular}




\begin{tabular}{|c|c|}
\hline C14-H14B & 0.9600 \\
\hline C14-H14C & 0.9600 \\
\hline C15-C16 & $1.525(6)$ \\
\hline C15-C17 & $1.529(5)$ \\
\hline C15-C18 & $1.547(5)$ \\
\hline C16-H16A & 0.9600 \\
\hline C16-H16B & 0.9600 \\
\hline C16-H16C & 0.9600 \\
\hline C17-H17A & 0.9600 \\
\hline C17-H17B & 0.9600 \\
\hline C17-H17C & 0.9600 \\
\hline C18-H18A & 0.9600 \\
\hline C18-H18B & 0.9600 \\
\hline C18-H18C & 0.9600 \\
\hline C19-H19A & 0.9700 \\
\hline C19-H19B & 0.9700 \\
\hline C19-C20 & $1.531(4)$ \\
\hline $\mathrm{C} 20-\mathrm{H} 20 \mathrm{~A}$ & 0.9700 \\
\hline C20-H20B & 0.9700 \\
\hline C20-C21 & $1.483(4)$ \\
\hline C21-H21 & 0.9300 \\
\hline C21-C22 & $1.295(5)$ \\
\hline $\mathrm{C} 22-\mathrm{H} 22 \mathrm{~A}$ & 0.9300 \\
\hline C22-H22B & 0.9300 \\
\hline C23-H23A & 0.9600 \\
\hline C23-H23B & 0.9600 \\
\hline $\mathrm{C} 23-\mathrm{H} 23 \mathrm{C}$ & 0.9600 \\
\hline S1A-C6A & $1.713(3)$ \\
\hline S1A-C7A & $1.713(3)$ \\
\hline Si2A-O3A & $1.6498(18)$ \\
\hline Si2A-C13A & $1.861(3)$ \\
\hline Si2A-C14A & $1.861(3)$ \\
\hline Si2A-C15A & $1.891(3)$ \\
\hline O1A-C1A & $1.411(3)$ \\
\hline O1A-C5A & $1.456(3)$ \\
\hline $\mathrm{O} 2 \mathrm{~A}-\mathrm{C} 1 \mathrm{~A}$ & $1.420(3)$ \\
\hline O2A-C9A & $1.437(3)$ \\
\hline $\mathrm{O} 3 \mathrm{~A}-\mathrm{C} 10 \mathrm{~A}$ & $1.425(3)$ \\
\hline N1A-C6A & $1.309(3)$ \\
\hline N1A-C8A & $1.376(3)$ \\
\hline $\mathrm{C} 1 \mathrm{~A}-\mathrm{C} 2 \mathrm{~A}$ & $1.544(3)$ \\
\hline C1A-C6A & $1.518(3)$ \\
\hline $\mathrm{C} 2 \mathrm{~A}-\mathrm{H} 2 \mathrm{~A}$ & 0.9800 \\
\hline $\mathrm{C} 2 \mathrm{~A}-\mathrm{C} 3 \mathrm{~A}$ & $1.544(4)$ \\
\hline
\end{tabular}




\begin{tabular}{|c|c|}
\hline C2A-C10A & $1.555(4)$ \\
\hline C3A-H3A & 0.9800 \\
\hline C3A-C4A & $1.544(4)$ \\
\hline C3A-C19A & $1.533(4)$ \\
\hline C4A-H4AA & 0.9700 \\
\hline C4A-H4AB & 0.9700 \\
\hline C4A-C5A & $1.510(4)$ \\
\hline C5A-H5A & 0.9800 \\
\hline $\mathrm{C} 5 \mathrm{~A}-\mathrm{C} 23 \mathrm{~A}$ & $1.513(4)$ \\
\hline C7A-H7A & 0.9300 \\
\hline C7A-C8A & $1.341(4)$ \\
\hline C8A-H8A & 0.9300 \\
\hline C9A-H9AA & 0.9600 \\
\hline C9A-H9AB & 0.9600 \\
\hline C9A-H9AC & 0.9600 \\
\hline C10A-H10A & 0.9800 \\
\hline C10A-C11A & $1.503(4)$ \\
\hline C11A-H11A & 0.9300 \\
\hline C11A-C12A & $1.313(4)$ \\
\hline C12A-H12C & 0.9300 \\
\hline C12A-H12D & 0.9300 \\
\hline C13A-H13D & 0.9600 \\
\hline C13A-H13E & 0.9600 \\
\hline C13A-H13F & 0.9600 \\
\hline C14A-H14D & 0.9600 \\
\hline C14A-H14E & 0.9600 \\
\hline C14A-H14F & 0.9600 \\
\hline C15A-C16A & $1.531(4)$ \\
\hline C15A-C17A & $1.526(4)$ \\
\hline C15A-C18A & $1.539(4)$ \\
\hline C16A-H16D & 0.9600 \\
\hline C16A-H16E & 0.9600 \\
\hline C16A-H16F & 0.9600 \\
\hline C17A-H17D & 0.9600 \\
\hline C17A-H17E & 0.9600 \\
\hline C17A-H17F & 0.9600 \\
\hline C18A-H18D & 0.9600 \\
\hline C18A-H18E & 0.9600 \\
\hline C18A-H18F & 0.9600 \\
\hline C19A-H19C & 0.9700 \\
\hline C19A-H19D & 0.9700 \\
\hline C19A-C20A & $1.535(4)$ \\
\hline C20A-H20C & 0.9700 \\
\hline C20A-H20D & 0.9700 \\
\hline
\end{tabular}




\begin{tabular}{|c|c|}
\hline C20A-C21A & $1.500(5)$ \\
\hline C21A-H21A & 0.9300 \\
\hline C21A-C22A & $1.253(6)$ \\
\hline C22A-H22C & 0.9300 \\
\hline C22A-H22D & 0.9300 \\
\hline C23A-H23D & 0.9600 \\
\hline C23A-H23E & 0.9600 \\
\hline C23A-H23F & 0.9600 \\
\hline C7-S1-C6 & $89.26(13)$ \\
\hline O3-Si2-C13 & $110.66(12)$ \\
\hline O3-Si2-C14 & $103.36(14)$ \\
\hline O3-Si2-C15 & $110.92(13)$ \\
\hline C13-Si2-C15 & $109.45(16)$ \\
\hline C14-Si2-C13 & $111.76(18)$ \\
\hline C14-Si2-C15 & $110.59(18)$ \\
\hline C1-O1-C5 & 113.71(19) \\
\hline $\mathrm{C} 1-\mathrm{O} 2-\mathrm{C} 9$ & $114.6(2)$ \\
\hline $\mathrm{C} 10-\mathrm{O} 3-\mathrm{Si} 2$ & $126.48(17)$ \\
\hline C6-N1-C8 & $110.2(2)$ \\
\hline $\mathrm{O} 1-\mathrm{C} 1-\mathrm{O} 2$ & $111.26(18)$ \\
\hline $\mathrm{O} 1-\mathrm{C} 1-\mathrm{C} 2$ & $113.47(19)$ \\
\hline $\mathrm{O} 1-\mathrm{C} 1-\mathrm{C} 6$ & $104.86(19)$ \\
\hline $\mathrm{O} 2-\mathrm{C} 1-\mathrm{C} 2$ & $105.46(19)$ \\
\hline $\mathrm{O} 2-\mathrm{C} 1-\mathrm{C} 6$ & $109.63(19)$ \\
\hline C6-C1-C2 & $112.24(19)$ \\
\hline $\mathrm{C} 1-\mathrm{C} 2-\mathrm{H} 2$ & 105.9 \\
\hline $\mathrm{C} 1-\mathrm{C} 2-\mathrm{C} 3$ & $112.32(19)$ \\
\hline $\mathrm{C} 1-\mathrm{C} 2-\mathrm{C} 10$ & $114.3(2)$ \\
\hline C3-C2-H2 & 105.9 \\
\hline $\mathrm{C} 3-\mathrm{C} 2-\mathrm{C} 10$ & $111.7(2)$ \\
\hline $\mathrm{C} 10-\mathrm{C} 2-\mathrm{H} 2$ & 105.9 \\
\hline C2-C3-H3 & 106.4 \\
\hline C4-C3-C2 & $111.5(2)$ \\
\hline C4-C3-H3 & 106.4 \\
\hline C19-C3-C2 & $113.1(2)$ \\
\hline C19-C3-H3 & 106.4 \\
\hline C19-C3-C4 & $112.5(2)$ \\
\hline C3-C4-H4A & 108.8 \\
\hline C3-C4-H4B & 108.8 \\
\hline H4A-C4-H4B & 107.7 \\
\hline C5-C4-C3 & $113.8(2)$ \\
\hline C5-C4-H4A & 108.8 \\
\hline C5-C4-H4B & 108.8 \\
\hline
\end{tabular}




$\begin{array}{ll}\text { O1-C5-C4 } & 109.87(19) \\ \text { O1-C5-H5 } & 109.6 \\ \text { O1-C5-C23 } & 105.3(2) \\ \text { C4-C5-H5 } & 109.6 \\ \text { C23-C5-C4 } & 112.8(2) \\ \text { C23-C5-H5 } & 109.6 \\ \text { N1-C6-S1 } & 114.8(2) \\ \text { N1-C6-C1 } & 123.3(2) \\ \text { C1-C6-S1 } & 121.85(17) \\ \text { S1-C7-H7 } & 125.0 \\ \text { C8-C7-S1 } & 110.0(2) \\ \text { C8-C7-H7 } & 125.0 \\ \text { N1-C8-H8 } & 122.1 \\ \text { C7-C8-N1 } & 115.7(2) \\ \text { C7-C8-H8 } & 122.1 \\ \text { O2-C9-H9A } & 109.5 \\ \text { O2-C9-H9B } & 109.5 \\ \text { O2-C9-H9C } & 109.5 \\ \text { H9A-C9-H9B } & 109.5 \\ \text { H9A-C9-H9C } & 109.5 \\ \text { H9B-C9-H9C } & 109.5 \\ \text { O3-C10-C2 } & 107.9(2) \\ \text { O3-C10-H10 } & 108.0 \\ \text { O3-C10-C11 } & 110.2(2) \\ \text { C2-C10-H10 } & 108.0 \\ \text { C11-C10-C2 } & 114.6(2) \\ \text { C11-C10-H10 } & 108.0 \\ \text { C10-C11-H11 } & 117.3 \\ \text { C12-C11-C10 } & 125.4(3) \\ \text { C12-C11-H11 } & 117.3 \\ \text { C11-C12-H12A } & 120.0 \\ \text { C11-C12-H12B } & 120.0 \\ \text { H12A-C12-H12B } & 120.0 \\ \text { Si2-C13-H13A } & 109.5 \\ \text { Si2-C13-H13B } & 109.5 \\ \text { Si2-C13-H13C } & 109.5 \\ \text { H13A-C13-H13B } & 109.5 \\ \text { H13A-C13-H13C } & 109.5 \\ \text { H13B-C13-H13C } & 109.5 \\ \text { Si2-C14-H14A } & 109.5 \\ \text { Si2-C14-H14B } & 109.5 \\ \text { Si2-C14-H14C } & 109.5 \\ \text { H14A-C14-H14B } & 109.5 \\ \text { H14A-C14-H14C } & 109.5 \\ & \\ & \end{array}$




$\begin{array}{ll}\text { H14B-C14-H14C } & 109.5 \\ \text { C16-C15-Si2 } & 110.6(2) \\ \text { C16-C15-C17 } & 108.8(4) \\ \text { C16-C15-C18 } & 109.9(3) \\ \text { C17-C15-Si2 } & 109.8(3) \\ \text { C17-C15-C18 } & 108.6(3) \\ \text { C18-C15-Si2 } & 109.0(3) \\ \text { C15-C16-H16A } & 109.5 \\ \text { C15-C16-H16B } & 109.5 \\ \text { C15-C16-H16C } & 109.5 \\ \text { H16A-C16-H16B } & 109.5 \\ \text { H16A-C16-H16C } & 109.5 \\ \text { H16B-C16-H16C } & 109.5 \\ \text { C15-C17-H17A } & 109.5 \\ \text { C15-C17-H17B } & 109.5 \\ \text { C15-C17-H17C } & 109.5 \\ \text { H17A-C17-H17B } & 109.5 \\ \text { H17A-C17-H17C } & 109.5 \\ \text { H17B-C17-H17C } & 109.5 \\ \text { C15-C18-H18A } & 109.5 \\ \text { C15-C18-H18B } & 109.5 \\ \text { C15-C18-H18C } & 109.5 \\ \text { H18A-C18-H18B } & 109.5 \\ \text { H18A-C18-H18C } & 109.5 \\ \text { H18B-C18-H18C } & 109.5 \\ \text { C3-C19-H19A } & 108.9 \\ \text { C3-C19-H19B } & 108.9 \\ \text { C3-C19-C20 } & 113.5(2) \\ \text { H19A-C19-H19B } & 107.7 \\ \text { C20-C19-H19A } & 108.9 \\ \text { C20-C19-H19B } & 108.9 \\ \text { C19-C20-H20A } & 108.7 \\ \text { C19-C20-H20B } & 108.7 \\ \text { H20A-C20-H20B } & 107.6 \\ \text { C21-C20-C19 } & 114.3(3) \\ \text { C21-C20-H20A } & 108.7 \\ \text { C21-C20-H20B } & 108.7 \\ \text { C20-C21-H21 } & 116.7 \\ \text { C22-C21-C20 } & 126.6(4) \\ \text { C22-C21-H21 } & 116.7 \\ \text { C21-C22-H22A } & 120.0 \\ \text { C21-C22-H22B } & 120.0 \\ \text { H22A-C22-H22B } & 120.0 \\ \text { C5-C23-H23A } & 109.5 \\ & \end{array}$




$\begin{array}{ll}\text { C5-C23-H23B } & 109.5 \\ \text { C5-C23-H23C } & 109.5 \\ \text { H23A-C23-H23B } & 109.5 \\ \text { H23A-C23-H23C } & 109.5 \\ \text { H23B-C23-H23C } & 109.5 \\ \text { C7A-S1A-C6A } & 89.34(13) \\ \text { O3A-Si2A-C13A } & 110.15(13) \\ \text { O3A-Si2A-C14A } & 102.82(13) \\ \text { O3A-Si2A-C15A } & 113.72(12) \\ \text { C13A-Si2A-C15A } & 109.47(14) \\ \text { C14A-Si2A-C13A } & 110.39(18) \\ \text { C14A-Si2A-C15A } & 110.13(16) \\ \text { C1A-O1A-C5A } & 112.96(19) \\ \text { C1A-O2A-C9A } & 114.4(2) \\ \text { C10A-O3A-Si2A } & 127.68(16) \\ \text { C6A-N1A-C8A } & 110.1(2) \\ \text { O1A-C1A-O2A } & 111.14(19) \\ \text { O1A-C1A-C2A } & 113.0(2) \\ \text { O1A-C1A-C6A } & 105.7(2) \\ \text { O2A-C1A-C2A } & 105.9(2) \\ \text { O2A-C1A-C6A } & 109.0(2) \\ \text { C6A-C1A-C2A } & 112.16(19) \\ \text { C1A-C2A-H2A } & 105.8 \\ \text { C1A-C2A-C10A } & 113.7(2) \\ \text { C3A-C2A-C1A } & 112.9(2) \\ \text { C3A-C2A-H2A } & 105.8 \\ \text { C3A-C2A-C10A } & 112.0(2) \\ \text { C10A-C2A-H2A } & 105.8 \\ \text { C2A-C3A-H3A } & 106.4 \\ \text { C2A-C3A-C4A } & 110.9(2) \\ \text { C4A-C3A-H3A } & 106.4 \\ \text { C19A-C3A-C2A } & 114.3(2) \\ \text { C19A-C3A-H3A } & 106.4 \\ \text { C19A-C3A-C4A } & 112.0(2) \\ \text { C3A-C4A-H4AA } & 108.6 \\ \text { C3A-C4A-H4AB } & 108.6 \\ \text { H4AA-C4A-H4AB } & 107.6 \\ \text { C5A-C4A-C3A } & 114.6(2) \\ \text { C5A-C4A-H4AA } & 108.6 \\ \text { C5A-C4A-H4AB } & 108.6 \\ & 110.0(2) \\ \text { O1A-C4A } & 109.4 \\ \text { OA } & 105.6(2) \\ \text { OA } & 109.4 \\ \end{array}$




\begin{tabular}{|c|c|}
\hline $\mathrm{C} 4 \mathrm{~A}-\mathrm{C} 5 \mathrm{~A}-\mathrm{C} 23 \mathrm{~A}$ & $113.1(3)$ \\
\hline C23A-C5A-H5A & 109.4 \\
\hline N1A-C6A-S1A & $114.7(2)$ \\
\hline N1A-C6A-C1A & $122.5(2)$ \\
\hline C1A-C6A-S1A & $122.72(18)$ \\
\hline S1A-C7A-H7A & 125.0 \\
\hline C8A-C7A-S1A & $110.1(2)$ \\
\hline C8A-C7A-H7A & 125.0 \\
\hline N1A-C8A-H8A & 122.1 \\
\hline C7A-C8A-N1A & $115.8(2)$ \\
\hline C7A-C8A-H8A & 122.1 \\
\hline O2A-C9A-H9AA & 109.5 \\
\hline O2A-C9A-H9AB & 109.5 \\
\hline O2A-C9A-H9AC & 109.5 \\
\hline H9AA-C9A-H9AB & 109.5 \\
\hline H9AA-C9A-H9AC & 109.5 \\
\hline H9AB-C9A-H9AC & 109.5 \\
\hline O3A-C10A-C2A & $108.1(2)$ \\
\hline O3A-C10A-H10A & 107.9 \\
\hline O3A-C10A-C11A & $109.2(2)$ \\
\hline C2A-C10A-H10A & 107.9 \\
\hline $\mathrm{C} 11 \mathrm{~A}-\mathrm{C} 10 \mathrm{~A}-\mathrm{C} 2 \mathrm{~A}$ & $115.7(2)$ \\
\hline C11A-C10A-H10A & 107.9 \\
\hline C10A-C11A-H11A & 117.5 \\
\hline C12A-C11A-C10A & $125.0(3)$ \\
\hline C12A-C11A-H11A & 117.5 \\
\hline $\mathrm{C} 11 \mathrm{~A}-\mathrm{C} 12 \mathrm{~A}-\mathrm{H} 12 \mathrm{C}$ & 120.0 \\
\hline C11A-C12A-H12D & 120.0 \\
\hline H12C-C12A-H12D & 120.0 \\
\hline Si2A-C13A-H13D & 109.5 \\
\hline Si2A-C13A-H13E & 109.5 \\
\hline Si2A-C13A-H13F & 109.5 \\
\hline H13D-C13A-H13E & 109.5 \\
\hline H13D-C13A-H13F & 109.5 \\
\hline H13E-C13A-H13F & 109.5 \\
\hline Si2A-C14A-H14D & 109.5 \\
\hline $\mathrm{Si} 2 \mathrm{~A}-\mathrm{C} 14 \mathrm{~A}-\mathrm{H} 14 \mathrm{E}$ & 109.5 \\
\hline Si2A-C14A-H14F & 109.5 \\
\hline H14D-C14A-H14E & 109.5 \\
\hline H14D-C14A-H14F & 109.5 \\
\hline H14E-C14A-H14F & 109.5 \\
\hline C16A-C15A-Si2A & $112.7(2)$ \\
\hline C16A-C15A-C18A & $107.5(3)$ \\
\hline C17A-C15A-Si2A & $110.8(2)$ \\
\hline
\end{tabular}




\begin{tabular}{|c|c|}
\hline C17A-C15A-C16A & $108.8(3)$ \\
\hline C17A-C15A-C18A & $109.1(2)$ \\
\hline C18A-C15A-Si2A & $107.8(2)$ \\
\hline C15A-C16A-H16D & 109.5 \\
\hline C15A-C16A-H16E & 109.5 \\
\hline C15A-C16A-H16F & 109.5 \\
\hline H16D-C16A-H16E & 109.5 \\
\hline H16D-C16A-H16F & 109.5 \\
\hline H16E-C16A-H16F & 109.5 \\
\hline C15A-C17A-H17D & 109.5 \\
\hline C15A-C17A-H17E & 109.5 \\
\hline C15A-C17A-H17F & 109.5 \\
\hline H17D-C17A-H17E & 109.5 \\
\hline H17D-C17A-H17F & 109.5 \\
\hline H17E-C17A-H17F & 109.5 \\
\hline C15A-C18A-H18D & 109.5 \\
\hline C15A-C18A-H18E & 109.5 \\
\hline C15A-C18A-H18F & 109.5 \\
\hline H18D-C18A-H18E & 109.5 \\
\hline H18D-C18A-H18F & 109.5 \\
\hline H18E-C18A-H18F & 109.5 \\
\hline C3A-C19A-H19C & 108.8 \\
\hline C3A-C19A-H19D & 108.8 \\
\hline C3A-C19A-C20A & $113.9(2)$ \\
\hline H19C-C19A-H19D & 107.7 \\
\hline C20A-C19A-H19C & 108.8 \\
\hline C20A-C19A-H19D & 108.8 \\
\hline C19A-C20A-H20C & 108.8 \\
\hline C19A-C20A-H20D & 108.8 \\
\hline H20C-C20A-H20D & 107.7 \\
\hline C21A-C20A-C19A & $113.8(3)$ \\
\hline C21A-C20A-H20C & 108.8 \\
\hline C21A-C20A-H20D & 108.8 \\
\hline C20A-C21A-H21A & 115.3 \\
\hline $\mathrm{C} 22 \mathrm{~A}-\mathrm{C} 21 \mathrm{~A}-\mathrm{C} 20 \mathrm{~A}$ & $129.5(4)$ \\
\hline $\mathrm{C} 22 \mathrm{~A}-\mathrm{C} 21 \mathrm{~A}-\mathrm{H} 21 \mathrm{~A}$ & 115.3 \\
\hline $\mathrm{C} 21 \mathrm{~A}-\mathrm{C} 22 \mathrm{~A}-\mathrm{H} 22 \mathrm{C}$ & 120.0 \\
\hline C21A-C22A-H22D & 120.0 \\
\hline $\mathrm{H} 22 \mathrm{C}-\mathrm{C} 22 \mathrm{~A}-\mathrm{H} 22 \mathrm{D}$ & 120.0 \\
\hline C5A-C23A-H23D & 109.5 \\
\hline C5A-C23A-H23E & 109.5 \\
\hline C5A-C23A-H23F & 109.5 \\
\hline $\mathrm{H} 23 \mathrm{D}-\mathrm{C} 23 \mathrm{~A}-\mathrm{H} 23 \mathrm{E}$ & 109.5 \\
\hline $\mathrm{H} 23 \mathrm{D}-\mathrm{C} 23 \mathrm{~A}-\mathrm{H} 23 \mathrm{~F}$ & 109.5 \\
\hline
\end{tabular}


Symmetry transformations used to generate equivalent atoms: 
Table 4. Anisotropic displacement parameters $\left(\AA^{2} \times 10^{3}\right)$ for 12 . The anisotropic displacement factor exponent takes the form: $\quad-2 p^{2}\left[\mathrm{~h}^{2} \mathrm{a}^{* 2} \mathrm{U}^{11}+\ldots+2 \mathrm{~h} \mathrm{k} \mathrm{a}^{*} \mathrm{~b}^{*} \mathrm{U}^{12}\right]$

\begin{tabular}{|c|c|c|c|c|c|c|}
\hline & U11 & U22 & U33 & $\mathrm{U} 23$ & U13 & U12 \\
\hline $\mathrm{S} 1$ & $25(1)$ & $41(1)$ & $44(1)$ & $-13(1)$ & $-8(1)$ & $7(1)$ \\
\hline $\mathrm{Si} 2$ & $40(1)$ & $27(1)$ & $37(1)$ & $4(1)$ & $-1(1)$ & $0(1)$ \\
\hline $\mathrm{O} 1$ & $24(1)$ & $24(1)$ & $27(1)$ & $-5(1)$ & $-2(1)$ & $3(1)$ \\
\hline $\mathrm{O} 2$ & $23(1)$ & $31(1)$ & $22(1)$ & $4(1)$ & $2(1)$ & $1(1)$ \\
\hline $\mathrm{O} 3$ & $47(1)$ & $26(1)$ & $31(1)$ & $5(1)$ & $-2(1)$ & $-2(1)$ \\
\hline N1 & $28(1)$ & $35(1)$ & $26(1)$ & $-7(1)$ & $-3(1)$ & $2(1)$ \\
\hline $\mathrm{C} 1$ & $20(1)$ & $25(1)$ & $24(1)$ & $-2(1)$ & $3(1)$ & $0(1)$ \\
\hline $\mathrm{C} 2$ & $24(1)$ & $24(1)$ & $23(1)$ & $-3(1)$ & $1(1)$ & $1(1)$ \\
\hline $\mathrm{C} 3$ & $27(1)$ & $28(1)$ & $21(1)$ & $2(1)$ & $-1(1)$ & $-1(1)$ \\
\hline $\mathrm{C} 4$ & $30(1)$ & $33(1)$ & $25(1)$ & $-5(1)$ & $-1(1)$ & $-3(1)$ \\
\hline C5 & $25(1)$ & $26(1)$ & $29(1)$ & $-5(1)$ & $-1(1)$ & $-2(1)$ \\
\hline C6 & $25(1)$ & $28(1)$ & $23(1)$ & $-2(1)$ & $-2(1)$ & $2(1)$ \\
\hline $\mathrm{C} 7$ & $31(2)$ & $46(2)$ & $36(2)$ & $-5(1)$ & $-9(1)$ & $-2(1)$ \\
\hline $\mathrm{C} 8$ & $32(2)$ & $40(1)$ & $28(1)$ & $-8(1)$ & $-6(1)$ & $-2(1)$ \\
\hline C9 & $38(2)$ & $42(2)$ & $36(2)$ & $15(1)$ & $4(1)$ & $2(1)$ \\
\hline $\mathrm{C} 10$ & $31(1)$ & $27(1)$ & $28(1)$ & $2(1)$ & $0(1)$ & $-4(1)$ \\
\hline C11 & $34(2)$ & $36(1)$ & $40(2)$ & $-2(1)$ & $9(1)$ & $-6(1)$ \\
\hline C12 & $64(2)$ & $50(2)$ & $47(2)$ & $-8(2)$ & $24(2)$ & $-15(2)$ \\
\hline C13 & $53(2)$ & $35(1)$ & $44(2)$ & $-1(1)$ & $8(2)$ & $4(1)$ \\
\hline C14 & $70(3)$ & $45(2)$ & $59(2)$ & $7(2)$ & $-15(2)$ & $12(2)$ \\
\hline C15 & $54(2)$ & $34(1)$ & $59(2)$ & $-5(1)$ & $7(2)$ & $-9(2)$ \\
\hline C16 & $42(2)$ & $58(2)$ & $108(4)$ & $-6(2)$ & $-9(2)$ & $-14(2)$ \\
\hline C17 & $88(3)$ & $58(2)$ & $79(3)$ & $4(2)$ & $35(3)$ & $-20(2)$ \\
\hline C18 & $75(3)$ & $34(2)$ & $109(4)$ & $-14(2)$ & $18(3)$ & $-17(2)$ \\
\hline C19 & $28(1)$ & $34(1)$ & $26(1)$ & $2(1)$ & $-4(1)$ & $-1(1)$ \\
\hline $\mathrm{C} 20$ & $31(2)$ & $40(2)$ & $47(2)$ & $1(1)$ & $-2(1)$ & $3(1)$ \\
\hline $\mathrm{C} 21$ & $34(2)$ & $54(2)$ & $49(2)$ & $12(2)$ & $-3(2)$ & $9(1)$ \\
\hline $\mathrm{C} 22$ & $55(3)$ & $91(3)$ & $71(3)$ & $20(2)$ & $21(2)$ & $29(2)$ \\
\hline $\mathrm{C} 23$ & $35(2)$ & $28(1)$ & $40(2)$ & $-8(1)$ & $0(1)$ & $3(1)$ \\
\hline S1A & $26(1)$ & $41(1)$ & $48(1)$ & $-18(1)$ & $7(1)$ & $-9(1)$ \\
\hline $\mathrm{Si} 2 \mathrm{~A}$ & $30(1)$ & $29(1)$ & $30(1)$ & $-2(1)$ & $0(1)$ & $-3(1)$ \\
\hline O1A & $26(1)$ & $28(1)$ & $35(1)$ & $-13(1)$ & $2(1)$ & $-2(1)$ \\
\hline $\mathrm{O} 2 \mathrm{~A}$ & $25(1)$ & $30(1)$ & $27(1)$ & $-1(1)$ & $1(1)$ & $1(1)$ \\
\hline $\mathrm{O} 3 \mathrm{~A}$ & $34(1)$ & $28(1)$ & $31(1)$ & $-1(1)$ & $1(1)$ & $-3(1)$ \\
\hline N1A & $26(1)$ & $31(1)$ & $29(1)$ & $-8(1)$ & $2(1)$ & $-1(1)$ \\
\hline $\mathrm{C} 1 \mathrm{~A}$ & $21(1)$ & $27(1)$ & $28(1)$ & $-7(1)$ & $-1(1)$ & $1(1)$ \\
\hline $\mathrm{C} 2 \mathrm{~A}$ & $24(1)$ & $27(1)$ & $25(1)$ & $-5(1)$ & $2(1)$ & $-2(1)$ \\
\hline C3A & $30(2)$ & $35(1)$ & $28(1)$ & $-2(1)$ & $3(1)$ & $4(1)$ \\
\hline $\mathrm{C} 4 \mathrm{~A}$ & $35(2)$ & $38(1)$ & $28(1)$ & $-11(1)$ & 1(1) & $5(1)$ \\
\hline
\end{tabular}




$\begin{array}{lllllll}\mathrm{C} 5 \mathrm{~A} & 28(1) & 32(1) & 36(1) & -14(1) & 1(1) & 3(1) \\ \mathrm{C} 6 \mathrm{~A} & 25(1) & 26(1) & 27(1) & -4(1) & 1(1) & -2(1) \\ \mathrm{C} 7 \mathrm{~A} & 27(1) & 38(1) & 40(2) & -5(1) & 7(1) & -1(1) \\ \mathrm{C} 8 \mathrm{~A} & 28(1) & 33(1) & 28(1) & -6(1) & 5(1) & 1(1) \\ \mathrm{C} 9 \mathrm{~A} & 38(2) & 37(1) & 36(2) & 5(1) & 5(1) & 6(1) \\ \mathrm{C} 10 \mathrm{~A} & 29(1) & 26(1) & 30(1) & -3(1) & 1(1) & 0(1) \\ \mathrm{C} 11 \mathrm{~A} & 30(1) & 32(1) & 43(2) & -5(1) & -8(1) & 1(1) \\ \mathrm{C} 12 \mathrm{~A} & 47(2) & 48(2) & 45(2) & -8(1) & -16(2) & 5(2) \\ \mathrm{C} 13 \mathrm{~A} & 44(2) & 50(2) & 50(2) & -19(2) & -11(2) & 9(2) \\ \mathrm{C} 14 \mathrm{~A} & 68(2) & 42(2) & 55(2) & -2(2) & 26(2) & -14(2) \\ \mathrm{C} 15 \mathrm{~A} & 36(2) & 29(1) & 38(2) & 2(1) & -3(1) & 3(1) \\ \mathrm{C} 16 \mathrm{~A} & 47(2) & 55(2) & 57(2) & 11(2) & 19(2) & 14(2) \\ \mathrm{C} 17 \mathrm{~A} & 44(2) & 45(2) & 59(2) & -7(2) & -13(2) & 7(2) \\ \mathrm{C} 18 \mathrm{~A} & 49(2) & 35(1) & 57(2) & -9(1) & -3(2) & 8(1) \\ \mathrm{C} 19 \mathrm{~A} & 27(1) & 35(1) & 40(2) & -2(1) & 7(1) & 2(1) \\ \mathrm{C} 20 \mathrm{~A} & 32(2) & 38(2) & 62(2) & 2(1) & 5(2) & -2(1) \\ \mathrm{C} 21 \mathrm{~A} & 36(2) & 48(2) & 86(3) & -6(2) & 5(2) & -2(2) \\ \mathrm{C} 22 \mathrm{~A} & 49(2) & 74(3) & 109(4) & -7(3) & -1(3) & -15(2) \\ \mathrm{C} 23 \mathrm{~A} & 45(2) & 41(2) & 54(2) & -25(1) & 3(2) & -2(1)\end{array}$


Table 5. Hydrogen coordinates $\left(\times 10^{4}\right)$ and isotropic displacement parameters $\left(\AA^{2} \times 10^{3}\right)$ for 12.

\begin{tabular}{|c|c|c|c|c|}
\hline & $\mathrm{x}$ & $\mathrm{y}$ & $\mathrm{Z}$ & $\mathrm{U}(\mathrm{eq})$ \\
\hline $\mathrm{H} 2$ & 8891 & 5293 & 4917 & 28 \\
\hline H3 & 9085 & 5583 & 5851 & 31 \\
\hline $\mathrm{H} 4 \mathrm{~A}$ & 7927 & 6565 & 5986 & 35 \\
\hline H4B & 9205 & 6916 & 5987 & 35 \\
\hline H5 & 8944 & 7385 & 5156 & 32 \\
\hline $\mathrm{H} 7$ & 4356 & 5645 & 3782 & 45 \\
\hline H8 & 5965 & 4844 & 3570 & 40 \\
\hline H9A & 8245 & 6771 & 3783 & 58 \\
\hline H9B & 9476 & 7141 & 3912 & 58 \\
\hline H9C & 8325 & 7393 & 4209 & 58 \\
\hline H10 & 6919 & 4900 & 5031 & 34 \\
\hline H11 & 6053 & 5883 & 5543 & 44 \\
\hline $\mathrm{H} 12 \mathrm{~A}$ & 6652 & 4900 & 6314 & 64 \\
\hline H12B & 5689 & 5563 & 6353 & 64 \\
\hline H13A & 8084 & 3971 & 4466 & 66 \\
\hline H13B & 8748 & 3208 & 4533 & 66 \\
\hline $\mathrm{H} 13 \mathrm{C}$ & 9413 & 3956 & 4654 & 66 \\
\hline H14A & 10044 & 3544 & 5746 & 87 \\
\hline H14B & 9452 & 2755 & 5712 & 87 \\
\hline $\mathrm{H} 14 \mathrm{C}$ & 9064 & 3313 & 6149 & 87 \\
\hline H16A & 5581 & 3916 & 5226 & 104 \\
\hline H16B & 5024 & 3130 & 5122 & 104 \\
\hline $\mathrm{H} 16 \mathrm{C}$ & 5954 & 3463 & 4731 & 104 \\
\hline H17A & 6979 & 2748 & 6159 & 112 \\
\hline H17B & 5648 & 2678 & 5980 & 112 \\
\hline $\mathrm{H} 17 \mathrm{C}$ & 6187 & 3463 & 6107 & 112 \\
\hline H18A & 7249 & 2326 & 4817 & 109 \\
\hline H18B & 6303 & 1979 & 5195 & 109 \\
\hline $\mathrm{H} 18 \mathrm{C}$ & 7635 & 2053 & 5371 & 109 \\
\hline H19A & 10851 & 6306 & 5681 & 35 \\
\hline H19B & 10522 & 6260 & 5090 & 35 \\
\hline $\mathrm{H} 20 \mathrm{~A}$ & 10926 & 4969 & 5696 & 47 \\
\hline $\mathrm{H} 20 \mathrm{~B}$ & 10755 & 4977 & 5092 & 47 \\
\hline H21 & 12811 & 5593 & 5552 & 55 \\
\hline $\mathrm{H} 22 \mathrm{~A}$ & 12649 & 4696 & 4701 & 87 \\
\hline $\mathrm{H} 22 \mathrm{~B}$ & 13843 & 5058 & 4940 & 87 \\
\hline $\mathrm{H} 23 \mathrm{~A}$ & 7381 & 8210 & 5272 & 52 \\
\hline $\mathrm{H} 23 \mathrm{~B}$ & 8032 & 8128 & 5808 & 52 \\
\hline $\mathrm{H} 23 \mathrm{C}$ & 6828 & 7709 & 5708 & 52 \\
\hline
\end{tabular}




\begin{tabular}{|c|c|c|c|}
\hline $\mathrm{H} 2 \mathrm{~A}$ & 3899 & 5624 & 2729 \\
\hline $\mathrm{H} 3 \mathrm{~A}$ & 4226 & 5385 & 1796 \\
\hline H4AA & 3116 & 4402 & 1605 \\
\hline $\mathrm{H} 4 \mathrm{AB}$ & 4393 & 4052 & 1639 \\
\hline H5A & 3991 & 3545 & 2443 \\
\hline H7A & -669 & 5205 & 3815 \\
\hline H8A & 964 & 5946 & 4086 \\
\hline H9AA & 3298 & 3461 & 3374 \\
\hline H9AB & 3021 & 4080 & 3786 \\
\hline H9AC & 4314 & 3756 & 3738 \\
\hline H10A & 1914 & 5987 & 2573 \\
\hline $\mathrm{H} 11 \mathrm{~A}$ & 1191 & 5062 & 1971 \\
\hline $\mathrm{H} 12 \mathrm{C}$ & 1910 & 6153 & 1293 \\
\hline H12D & 983 & 5491 & 1172 \\
\hline H13D & 3655 & 6972 & 3169 \\
\hline $\mathrm{H} 13 \mathrm{E}$ & 4014 & 7801 & 3062 \\
\hline $\mathrm{H} 13 \mathrm{~F}$ & 4778 & 7151 & 2829 \\
\hline H14D & 4497 & 7632 & 1700 \\
\hline $\mathrm{H} 14 \mathrm{E}$ & 3921 & 8360 & 1924 \\
\hline $\mathrm{H} 14 \mathrm{~F}$ & 3240 & 7891 & 1505 \\
\hline H16D & 803 & 6857 & 2911 \\
\hline H16E & 254 & 7631 & 3059 \\
\hline $\mathrm{H} 16 \mathrm{~F}$ & 1528 & 7414 & 3257 \\
\hline H17D & 980 & 7852 & 1728 \\
\hline $\mathrm{H} 17 \mathrm{E}$ & -92 & 7830 & 2119 \\
\hline $\mathrm{H} 17 \mathrm{~F}$ & 562 & 7091 & 1965 \\
\hline H18D & 2237 & 8623 & 2872 \\
\hline H18E & 936 & 8773 & 2675 \\
\hline $\mathrm{H} 18 \mathrm{~F}$ & 2006 & 8790 & 2283 \\
\hline $\mathrm{H} 19 \mathrm{C}$ & 6001 & 4723 & 1960 \\
\hline H19D & 5598 & 4611 & 2538 \\
\hline $\mathrm{H} 20 \mathrm{C}$ & 6076 & 6026 & 2097 \\
\hline H20D & 5692 & 5907 & 2676 \\
\hline $\mathrm{H} 21 \mathrm{~A}$ & 7577 & 5264 & 2799 \\
\hline $\mathrm{H} 22 \mathrm{C}$ & 8216 & 6337 & 2131 \\
\hline $\mathrm{H} 22 \mathrm{D}$ & 9061 & 5869 & 2517 \\
\hline $\mathrm{H} 23 \mathrm{D}$ & 2445 & 2728 & 2258 \\
\hline $\mathrm{H} 23 \mathrm{E}$ & 3207 & 2826 & 1752 \\
\hline $\mathrm{H} 23 \mathrm{~F}$ & 1988 & 3244 & 1809 \\
\hline
\end{tabular}


Table 6. Torsion angles $\left[{ }^{\circ}\right]$ for 12.

\begin{tabular}{|c|c|}
\hline S1-C7-C8-N1 & $0.6(3)$ \\
\hline $\mathrm{Si2}-\mathrm{O} 3-\mathrm{C} 10-\mathrm{C} 2$ & $-98.8(2)$ \\
\hline $\mathrm{Si2}-\mathrm{O} 3-\mathrm{C} 10-\mathrm{C} 11$ & $135.4(2)$ \\
\hline $\mathrm{O} 1-\mathrm{C} 1-\mathrm{C} 2-\mathrm{C} 3$ & $-48.2(3)$ \\
\hline $\mathrm{O} 1-\mathrm{C} 1-\mathrm{C} 2-\mathrm{C} 10$ & $80.5(3)$ \\
\hline O1-C1-C6-S1 & $-4.5(3)$ \\
\hline O1-C1-C6-N1 & $178.2(2)$ \\
\hline $\mathrm{O} 2-\mathrm{C} 1-\mathrm{C} 2-\mathrm{C} 3$ & $73.8(2)$ \\
\hline $\mathrm{O} 2-\mathrm{C} 1-\mathrm{C} 2-\mathrm{C} 10$ & $-157.5(2)$ \\
\hline O2-C1-C6-S1 & $-124.0(2)$ \\
\hline O2-C1-C6-N1 & $58.7(3)$ \\
\hline O3-Si2-C15-C16 & $59.2(3)$ \\
\hline O3-Si2-C15-C17 & $-60.9(3)$ \\
\hline O3-Si2-C15-C18 & $-179.8(3)$ \\
\hline O3-C10-C11-C12 & $1.0(4)$ \\
\hline C1-O1-C5-C4 & $-60.5(3)$ \\
\hline $\mathrm{C} 1-\mathrm{O} 1-\mathrm{C} 5-\mathrm{C} 23$ & $177.8(2)$ \\
\hline C1-C2-C3-C4 & $41.3(3)$ \\
\hline C1-C2-C3-C19 & $-86.7(3)$ \\
\hline $\mathrm{C} 1-\mathrm{C} 2-\mathrm{C} 10-\mathrm{O} 3$ & $169.58(19)$ \\
\hline $\mathrm{C} 1-\mathrm{C} 2-\mathrm{C} 10-\mathrm{C} 11$ & $-67.3(3)$ \\
\hline C2-C1-C6-S1 & $119.1(2)$ \\
\hline C2-C1-C6-N1 & $-58.2(3)$ \\
\hline $\mathrm{C} 2-\mathrm{C} 3-\mathrm{C} 4-\mathrm{C} 5$ & $-45.5(3)$ \\
\hline C2-C3-C19-C20 & $-70.9(3)$ \\
\hline C2-C10-C11-C12 & $-120.9(3)$ \\
\hline $\mathrm{C} 3-\mathrm{C} 2-\mathrm{C} 10-\mathrm{O} 3$ & $-61.5(3)$ \\
\hline C3-C2-C10-C11 & $61.7(3)$ \\
\hline C3-C4-C5-O1 & $53.7(3)$ \\
\hline C3-C4-C5-C23 & $170.9(2)$ \\
\hline C3-C19-C20-C21 & $-172.5(3)$ \\
\hline C4-C3-C19-C20 & $161.7(2)$ \\
\hline $\mathrm{C} 5-\mathrm{O} 1-\mathrm{C} 1-\mathrm{O} 2$ & $-60.1(2)$ \\
\hline $\mathrm{C} 5-\mathrm{O} 1-\mathrm{C} 1-\mathrm{C} 2$ & $58.6(3)$ \\
\hline $\mathrm{C} 5-\mathrm{O} 1-\mathrm{C} 1-\mathrm{C} 6$ & $-178.57(19)$ \\
\hline C6-S1-C7-C8 & $-0.6(2)$ \\
\hline C6-N1-C8-C7 & $-0.2(4)$ \\
\hline $\mathrm{C} 6-\mathrm{C} 1-\mathrm{C} 2-\mathrm{C} 3$ & $-166.8(2)$ \\
\hline C6-C1-C2-C10 & $-38.2(3)$ \\
\hline C7-S1-C6-N1 & $0.5(2)$ \\
\hline C7-S1-C6-C1 & $-177.1(2)$ \\
\hline C8-N1-C6-S1 & $-0.2(3)$ \\
\hline
\end{tabular}




\begin{tabular}{|c|c|}
\hline C8-N1-C6-C1 & $177.3(2)$ \\
\hline $\mathrm{C} 9-\mathrm{O} 2-\mathrm{C} 1-\mathrm{O} 1$ & $-57.5(3)$ \\
\hline $\mathrm{C} 9-\mathrm{O} 2-\mathrm{C} 1-\mathrm{C} 2$ & $179.0(2)$ \\
\hline C9-O2-C1-C6 & $58.0(3)$ \\
\hline $\mathrm{C} 10-\mathrm{C} 2-\mathrm{C} 3-\mathrm{C} 4$ & $-88.8(2)$ \\
\hline C10-C2-C3-C19 & $143.3(2)$ \\
\hline C13-Si2-O3-C10 & $41.0(3)$ \\
\hline C13-Si2-C15-C16 & $-63.2(3)$ \\
\hline C13-Si2-C15-C17 & $176.7(3)$ \\
\hline C13-Si2-C15-C18 & $57.8(3)$ \\
\hline C14-Si2-O3-C10 & $160.8(2)$ \\
\hline C14-Si2-C15-C16 & $173.3(3)$ \\
\hline C14-Si2-C15-C17 & $53.2(3)$ \\
\hline C14-Si2-C15-C18 & $-65.8(3)$ \\
\hline C15-Si2-O3-C10 & $-80.6(2)$ \\
\hline C19-C3-C4-C5 & $82.7(3)$ \\
\hline C19-C20-C21-C22 & $-126.8(4)$ \\
\hline S1A-C7A-C8A-N1A & $0.7(3)$ \\
\hline Si2A-O3A-C10A-C2A & $-104.2(2)$ \\
\hline $\mathrm{Si2A}-\mathrm{O} 3 \mathrm{~A}-\mathrm{C} 10 \mathrm{~A}-\mathrm{C} 11 \mathrm{~A}$ & $129.3(2)$ \\
\hline O1A-C1A-C2A-C3A & $-49.4(3)$ \\
\hline O1A-C1A-C2A-C10A & $79.6(3)$ \\
\hline O1A-C1A-C6A-S1A & $-4.4(3)$ \\
\hline O1A-C1A-C6A-N1A & $174.1(2)$ \\
\hline $\mathrm{O} 2 \mathrm{~A}-\mathrm{C} 1 \mathrm{~A}-\mathrm{C} 2 \mathrm{~A}-\mathrm{C} 3 \mathrm{~A}$ & $72.5(2)$ \\
\hline $\mathrm{O} 2 \mathrm{~A}-\mathrm{C} 1 \mathrm{~A}-\mathrm{C} 2 \mathrm{~A}-\mathrm{C} 10 \mathrm{~A}$ & $-158.53(19)$ \\
\hline O2A-C1A-C6A-S1A & $-123.9(2)$ \\
\hline O2A-C1A-C6A-N1A & $54.6(3)$ \\
\hline O3A-Si2A-C15A-C16A & $75.0(3)$ \\
\hline O3A-Si2A-C15A-C17A & $-47.1(2)$ \\
\hline O3A-Si2A-C15A-C18A & $-166.46(19)$ \\
\hline O3A-C10A-C11A-C12A & $1.3(4)$ \\
\hline C1A-O1A-C5A-C4A & $-60.8(3)$ \\
\hline C1A-O1A-C5A-C23A & $176.9(2)$ \\
\hline C1A-C2A-C3A-C4A & $41.0(3)$ \\
\hline C1A-C2A-C3A-C19A & $-86.7(3)$ \\
\hline C1A-C2A-C10A-O3A & $166.46(19)$ \\
\hline $\mathrm{C} 1 \mathrm{~A}-\mathrm{C} 2 \mathrm{~A}-\mathrm{C} 10 \mathrm{~A}-\mathrm{C} 11 \mathrm{~A}$ & $-70.9(3)$ \\
\hline C2A-C1A-C6A-S1A & $119.2(2)$ \\
\hline $\mathrm{C} 2 \mathrm{~A}-\mathrm{C} 1 \mathrm{~A}-\mathrm{C} 6 \mathrm{~A}-\mathrm{N} 1 \mathrm{~A}$ & $-62.4(3)$ \\
\hline $\mathrm{C} 2 \mathrm{~A}-\mathrm{C} 3 \mathrm{~A}-\mathrm{C} 4 \mathrm{~A}-\mathrm{C} 5 \mathrm{~A}$ & $-44.5(3)$ \\
\hline C2A-C3A-C19A-C20A & $-62.8(3)$ \\
\hline $\mathrm{C} 2 \mathrm{~A}-\mathrm{C} 10 \mathrm{~A}-\mathrm{C} 11 \mathrm{~A}-\mathrm{C} 12 \mathrm{~A}$ & $-120.9(3)$ \\
\hline C3A-C2A-C10A-O3A & $-64.2(3)$ \\
\hline
\end{tabular}




\begin{tabular}{|c|c|}
\hline C3A-C2A-C10A-C11A & $58.5(3)$ \\
\hline C3A-C4A-C5A-O1A & $53.3(3)$ \\
\hline C3A-C4A-C5A-C23A & $171.0(2)$ \\
\hline C3A-C19A-C20A-C21A & $-179.1(3)$ \\
\hline C4A-C3A-C19A-C20A & $170.1(2)$ \\
\hline C5A-O1A-C1A-O2A & $-59.4(3)$ \\
\hline C5A-O1A-C1A-C2A & $59.5(3)$ \\
\hline C5A-O1A-C1A-C6A & $-177.4(2)$ \\
\hline C6A-S1A-C7A-C8A & $-0.8(2)$ \\
\hline C6A-N1A-C8A-C7A & $-0.1(3)$ \\
\hline C6A-C1A-C2A-C3A & $-168.7(2)$ \\
\hline C6A-C1A-C2A-C10A & $-39.8(3)$ \\
\hline C7A-S1A-C6A-N1A & $0.7(2)$ \\
\hline C7A-S1A-C6A-C1A & $179.3(2)$ \\
\hline C8A-N1A-C6A-S1A & $-0.5(3)$ \\
\hline C8A-N1A-C6A-C1A & $-179.0(2)$ \\
\hline C9A-O2A-C1A-O1A & $-59.5(3)$ \\
\hline $\mathrm{C} 9 \mathrm{~A}-\mathrm{O} 2 \mathrm{~A}-\mathrm{C} 1 \mathrm{~A}-\mathrm{C} 2 \mathrm{~A}$ & $177.5(2)$ \\
\hline C9A-O2A-C1A-C6A & $56.6(3)$ \\
\hline C10A-C2A-C3A-C4A & $-88.8(3)$ \\
\hline C10A-C2A-C3A-C19A & $143.5(2)$ \\
\hline C13A-Si2A-O3A-C10A & $68.2(3)$ \\
\hline C13A-Si2A-C15A-C16A & $-48.7(3)$ \\
\hline C13A-Si2A-C15A-C17A & $-170.8(2)$ \\
\hline C13A-Si2A-C15A-C18A & $69.9(2)$ \\
\hline C14A-Si2A-O3A-C10A & $-174.1(2)$ \\
\hline C14A-Si2A-C15A-C16A & $-170.2(2)$ \\
\hline C14A-Si2A-C15A-C17A & $67.7(2)$ \\
\hline C14A-Si2A-C15A-C18A & $-51.7(3)$ \\
\hline C15A-Si2A-O3A-C10A & $-55.1(2)$ \\
\hline C19A-C3A-C4A-C5A & $84.4(3)$ \\
\hline C19A-C20A-C21A-C22A & $128.5(5)$ \\
\hline
\end{tabular}

Symmetry transformations used to generate equivalent atoms:

Table 7. Hydrogen bonds for $\mathbf{1 2}\left[\AA\right.$ and $\left.^{\circ}\right]$.

\begin{tabular}{lllll}
\hline D-H...A & d(D-H) & d(H...A $)$ & d(D...A $)$ & $<($ DHA $)$
\end{tabular}


The X-ray structure of compound 15
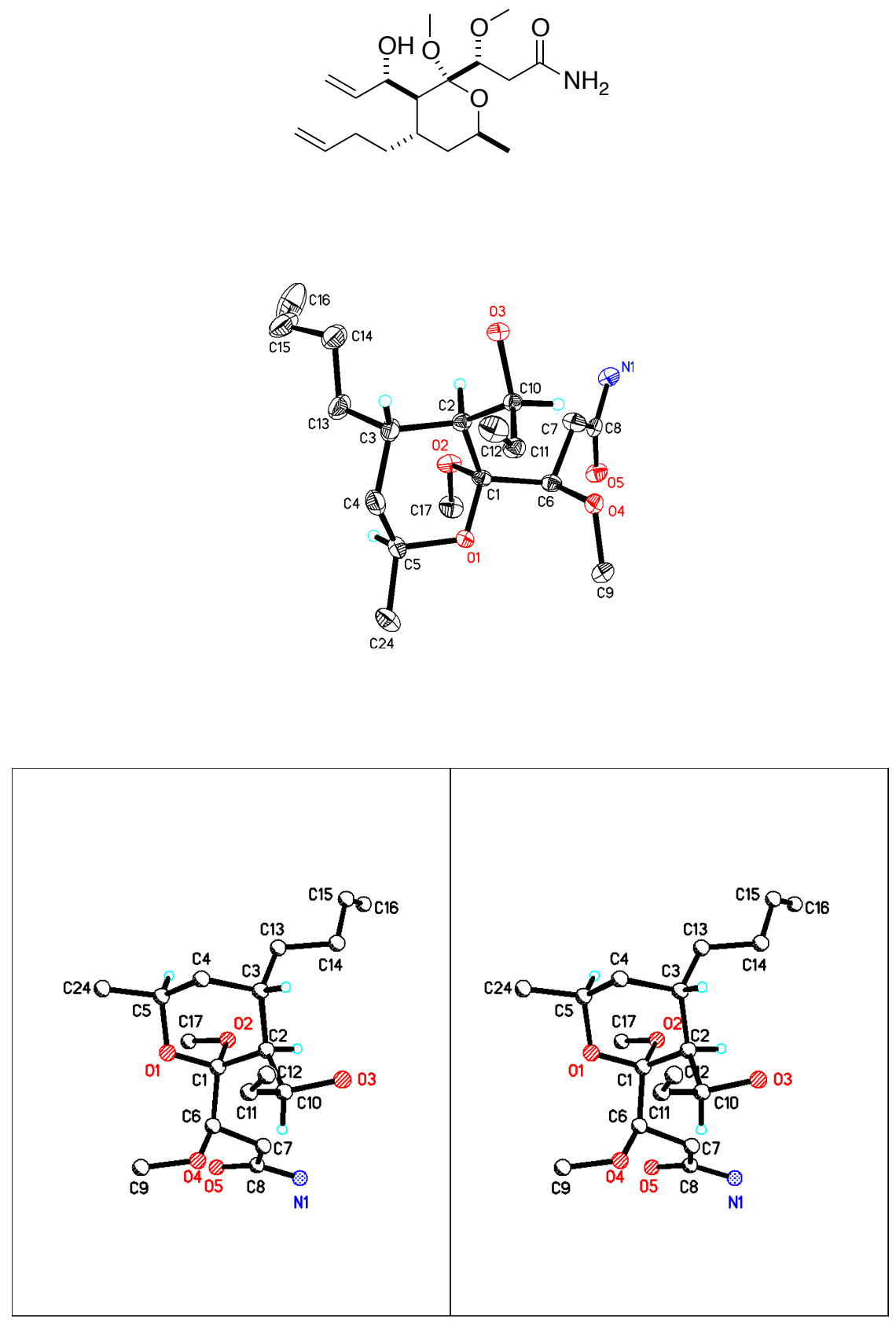
Table 1. Crystal data and structure refinement for $\mathbf{1 5 .}$

Identification code

Empirical formula

Formula weight

Temperature

Wavelength

Crystal system

Space group

Unit cell dimensions

Volume

Z

Density (calculated)

Absorption coefficient

$\mathrm{F}(000)$

Crystal size

Theta range for data collection

Index ranges

Reflections collected

Independent reflections

Completeness to theta $=67.684^{\circ}$

Absorption correction

Max. and min. transmission

Refinement method

Data / restraints / parameters

Goodness-of-fit on $\mathrm{F}^{2}$

Final $\mathrm{R}$ indices [I $>2 \operatorname{sigma}(\mathrm{I})]$

$\mathrm{R}$ indices (all data)

Absolute structure parameter

Extinction coefficient

Largest diff. peak and hole
15

$\mathrm{C}_{18} \mathrm{H}_{31} \mathrm{~N} \mathrm{O}_{5}$

341.44

180.01(10) K

$1.54184 \AA$

Orthorhombic

P 212121

$\mathrm{a}=7.71375(6) \AA \quad \mathrm{a}=90^{\circ}$.

$\mathrm{b}=8.88494(9) \AA \quad \mathrm{b}=90^{\circ}$.

$\mathrm{c}=28.0459(2) \AA \quad \mathrm{g}=90^{\circ}$.

$1922.16(3) \AA^{3}$

4

$1.180 \mathrm{Mg} / \mathrm{m}^{3}$

$0.693 \mathrm{~mm}^{-1}$

744

$0.15 \times 0.1 \times 0.1 \mathrm{~mm}^{3}$

5.222 to $66.593^{\circ}$.

$-9<=\mathrm{h}<=9,-10<=\mathrm{k}<=10,-33<=\mathrm{l}<=33$

41233

$3390[\mathrm{R}($ int $)=0.0383]$

$97.5 \%$

Semi-empirical from equivalents

1.00000 and 0.45532

Full-matrix least-squares on $\mathrm{F}^{2}$

$3390 / 0 / 229$

1.075

$\mathrm{R} 1=0.0372, \mathrm{wR} 2=0.1035$

$\mathrm{R} 1=0.0378, \mathrm{wR} 2=0.1042$

$-0.01(5)$

$\mathrm{n} / \mathrm{a}$

0.419 and -0.207 e. $\AA^{-3}$ 
Table 2. Atomic coordinates $\left(\mathrm{x}_{10} 0^{4}\right)$ and equivalent isotropic displacement parameters $\left(\AA^{2} \mathrm{x}\right.$ $10^{3}$ ) for 15 . $U(\mathrm{eq})$ is defined as one third of the trace of the orthogonalized $\mathrm{U}^{\mathrm{ij}}$ tensor.

\begin{tabular}{|c|c|c|c|c|}
\hline & $\mathrm{x}$ & $\mathrm{y}$ & $\mathrm{z}$ & $\mathrm{U}(\mathrm{eq})$ \\
\hline $\mathrm{C} 1$ & $7214(3)$ & $7548(3)$ & $8624(1)$ & $25(1)$ \\
\hline $\mathrm{C} 2$ & $6611(3)$ & $5904(3)$ & $8535(1)$ & $26(1)$ \\
\hline $\mathrm{C} 3$ & $6071(3)$ & $5087(3)$ & $9004(1)$ & $35(1)$ \\
\hline $\mathrm{C} 4$ & $5034(4)$ & $6126(3)$ & $9334(1)$ & $40(1)$ \\
\hline $\mathrm{C} 5$ & $5927(4)$ & $7620(3)$ & $9404(1)$ & $35(1)$ \\
\hline C6 & $7362(3)$ & $8588(3)$ & $8182(1)$ & $28(1)$ \\
\hline $\mathrm{C} 7$ & $8476(3)$ & $7869(3)$ & $7794(1)$ & $35(1)$ \\
\hline $\mathrm{C} 8$ & $10069(3)$ & $8767(2)$ & $7655(1)$ & $26(1)$ \\
\hline C9 & $4938(4)$ & $10316(3)$ & $8172(1)$ & $42(1)$ \\
\hline $\mathrm{C} 10$ & $5239(3)$ & $5703(2)$ & $8139(1)$ & $24(1)$ \\
\hline $\mathrm{C} 11$ & $3419(3)$ & $6091(3)$ & $8281(1)$ & $29(1)$ \\
\hline $\mathrm{C} 12$ & $2142(3)$ & $5120(3)$ & $8330(1)$ & $40(1)$ \\
\hline $\mathrm{C} 13$ & $7592(4)$ & $4385(3)$ & $9280(1)$ & $49(1)$ \\
\hline $\mathrm{C} 14$ & $8406(5)$ & $3018(4)$ & $9066(1)$ & $54(1)$ \\
\hline $\mathrm{C} 15$ & $9795(6)$ & $2400(4)$ & $9392(2)$ & $67(1)$ \\
\hline $\mathrm{C} 16$ & $11376(7)$ & $2292(6)$ & $9302(2)$ & $103(2)$ \\
\hline $\mathrm{C} 17$ & $9789(4)$ & $8663(3)$ & 8992(1) & $43(1)$ \\
\hline $\mathrm{C} 24$ & $4907(5)$ & $8726(4)$ & $9703(1)$ & $51(1)$ \\
\hline N1 & $11088(3)$ & $8059(2)$ & $7339(1)$ & $34(1)$ \\
\hline $\mathrm{O} 1$ & $6157(2)$ & $8339(2)$ & $8946(1)$ & $28(1)$ \\
\hline $\mathrm{O} 2$ & $8924(2)$ & $7347(2)$ & $8812(1)$ & $35(1)$ \\
\hline $\mathrm{O} 3$ & $5392(2)$ & $4162(2)$ & $7990(1)$ & $28(1)$ \\
\hline $\mathrm{O} 4$ & $5709(2)$ & $8978(2)$ & $7981(1)$ & $30(1)$ \\
\hline O5 & $10398(2)$ & $10019(2)$ & $7815(1)$ & $36(1)$ \\
\hline
\end{tabular}


Table 3. Bond lengths $[\AA]$ and angles $\left[{ }^{\circ}\right]$ for $\mathbf{1 5}$.

\begin{tabular}{|c|c|}
\hline $\mathrm{C} 1-\mathrm{C} 2$ & $1.553(3)$ \\
\hline C1-C6 & $1.549(3)$ \\
\hline $\mathrm{C} 1-\mathrm{O} 1$ & $1.405(3)$ \\
\hline $\mathrm{C} 1-\mathrm{O} 2$ & $1.433(3)$ \\
\hline $\mathrm{C} 2-\mathrm{H} 2$ & 1.0000 \\
\hline $\mathrm{C} 2-\mathrm{C} 3$ & $1.559(3)$ \\
\hline $\mathrm{C} 2-\mathrm{C} 10$ & $1.545(3)$ \\
\hline C3-H3 & 1.0000 \\
\hline C3-C4 & $1.531(4)$ \\
\hline $\mathrm{C} 3-\mathrm{C} 13$ & $1.537(4)$ \\
\hline C4-H4A & 0.9900 \\
\hline C4-H4B & 0.9900 \\
\hline C4-C5 & $1.509(4)$ \\
\hline C5-H5 & 1.0000 \\
\hline C5-C24 & $1.512(4)$ \\
\hline C5-O1 & $1.445(3)$ \\
\hline C6-H6 & 1.0000 \\
\hline C6-C7 & $1.526(3)$ \\
\hline C6-O4 & $1.437(3)$ \\
\hline C7-H7A & 0.9900 \\
\hline C7-H7B & 0.9900 \\
\hline C7-C8 & $1.516(3)$ \\
\hline C8-N1 & $1.342(3)$ \\
\hline C8-O5 & $1.226(3)$ \\
\hline C9-H9A & 0.9800 \\
\hline C9-H9B & 0.9800 \\
\hline C9-H9C & 0.9800 \\
\hline C9-O4 & $1.432(3)$ \\
\hline C10-H10 & 1.0000 \\
\hline C10-C11 & $1.499(3)$ \\
\hline $\mathrm{C} 10-\mathrm{O} 3$ & $1.437(3)$ \\
\hline C11-H11 & 0.9500 \\
\hline C11-C12 & $1.316(4)$ \\
\hline C12-H12A & 0.9500 \\
\hline C12-H12B & 0.9500 \\
\hline C13-H13A & 0.9900 \\
\hline C13-H13B & 0.9900 \\
\hline C13-C14 & $1.493(4)$ \\
\hline C14-H14A & 0.9900 \\
\hline C14-H14B & 0.9900 \\
\hline C14-C15 & $1.511(5)$ \\
\hline C15-H15 & 0.9500 \\
\hline
\end{tabular}




\begin{tabular}{|c|c|}
\hline C15-C16 & $1.249(7)$ \\
\hline C16-H16A & 0.9500 \\
\hline C16-H16B & 0.9500 \\
\hline C17-H17A & 0.9800 \\
\hline C17-H17B & 0.9800 \\
\hline C17-H17C & 0.9800 \\
\hline $\mathrm{C} 17-\mathrm{O} 2$ & $1.437(3)$ \\
\hline C24-H24A & 0.9800 \\
\hline C24-H24B & 0.9800 \\
\hline C24-H24C & 0.9800 \\
\hline N1-H1A & $0.92(3)$ \\
\hline N1-H1B & $0.92(4)$ \\
\hline O3-H3A & 0.8400 \\
\hline C6-C1-C2 & $117.10(17)$ \\
\hline $\mathrm{O} 1-\mathrm{C} 1-\mathrm{C} 2$ & $113.55(18)$ \\
\hline O1-C1-C6 & $104.97(17)$ \\
\hline $\mathrm{O} 1-\mathrm{C} 1-\mathrm{O} 2$ & $111.07(17)$ \\
\hline $\mathrm{O} 2-\mathrm{C} 1-\mathrm{C} 2$ & $102.53(17)$ \\
\hline $\mathrm{O} 2-\mathrm{C} 1-\mathrm{C} 6$ & $107.55(18)$ \\
\hline $\mathrm{C} 1-\mathrm{C} 2-\mathrm{H} 2$ & 105.4 \\
\hline $\mathrm{C} 1-\mathrm{C} 2-\mathrm{C} 3$ & $112.52(19)$ \\
\hline C3-C2-H2 & 105.4 \\
\hline $\mathrm{C} 10-\mathrm{C} 2-\mathrm{C} 1$ & $115.37(18)$ \\
\hline $\mathrm{C} 10-\mathrm{C} 2-\mathrm{H} 2$ & 105.4 \\
\hline $\mathrm{C} 10-\mathrm{C} 2-\mathrm{C} 3$ & 111.71(19) \\
\hline C2-C3-H3 & 106.9 \\
\hline $\mathrm{C} 4-\mathrm{C} 3-\mathrm{C} 2$ & $111.6(2)$ \\
\hline C4-C3-H3 & 106.9 \\
\hline C4-C3-C13 & $109.9(2)$ \\
\hline $\mathrm{C} 13-\mathrm{C} 3-\mathrm{C} 2$ & $114.2(2)$ \\
\hline C13-C3-H3 & 106.9 \\
\hline C3-C4-H4A & 109.3 \\
\hline C3-C4-H4B & 109.3 \\
\hline H4A-C4-H4B & 107.9 \\
\hline C5-C4-C3 & $111.7(2)$ \\
\hline C5-C4-H4A & 109.3 \\
\hline C5-C4-H4B & 109.3 \\
\hline C4-C5-H5 & 109.3 \\
\hline $\mathrm{C} 4-\mathrm{C} 5-\mathrm{C} 24$ & $114.0(2)$ \\
\hline C24-C5-H5 & 109.3 \\
\hline O1-C5-C4 & $109.22(19)$ \\
\hline O1-C5-H5 & 109.3 \\
\hline O1-C5-C24 & $105.6(2)$ \\
\hline
\end{tabular}




\begin{tabular}{|c|c|}
\hline C1-C6-H6 & 107.9 \\
\hline C7-C6-C1 & $111.22(19)$ \\
\hline C7-C6-H6 & 107.9 \\
\hline O4-C6-C1 & $113.05(17)$ \\
\hline O4-C6-H6 & 107.9 \\
\hline O4-C6-C7 & $108.70(19)$ \\
\hline C6-C7-H7A & 108.6 \\
\hline C6-C7-H7B & 108.6 \\
\hline H7A-C7-H7B & 107.5 \\
\hline C8-C7-C6 & $114.8(2)$ \\
\hline C8-C7-H7A & 108.6 \\
\hline C8-C7-H7B & 108.6 \\
\hline N1-C8-C7 & $113.5(2)$ \\
\hline $\mathrm{O} 5-\mathrm{C} 8-\mathrm{C} 7$ & $123.4(2)$ \\
\hline O5-C8-N1 & $123.1(2)$ \\
\hline H9A-C9-H9B & 109.5 \\
\hline H9A-C9-H9C & 109.5 \\
\hline H9B-C9-H9C & 109.5 \\
\hline O4-C9-H9A & 109.5 \\
\hline O4-C9-H9B & 109.5 \\
\hline O4-C9-H9C & 109.5 \\
\hline C2-C10-H10 & 108.1 \\
\hline C11-C10-C2 & $115.13(18)$ \\
\hline C11-C10-H10 & 108.1 \\
\hline $\mathrm{O} 3-\mathrm{C} 10-\mathrm{C} 2$ & $105.28(17)$ \\
\hline O3-C10-H10 & 108.1 \\
\hline $\mathrm{O} 3-\mathrm{C} 10-\mathrm{C} 11$ & $111.91(18)$ \\
\hline C10-C11-H11 & 117.4 \\
\hline C12-C11-C10 & $125.3(2)$ \\
\hline C12-C11-H11 & 117.4 \\
\hline C11-C12-H12A & 120.0 \\
\hline C11-C12-H12B & 120.0 \\
\hline H12A-C12-H12B & 120.0 \\
\hline C3-C13-H13A & 108.1 \\
\hline C3-C13-H13B & 108.1 \\
\hline H13A-C13-H13B & 107.3 \\
\hline C14-C13-C3 & $116.7(2)$ \\
\hline C14-C13-H13A & 108.1 \\
\hline C14-C13-H13B & 108.1 \\
\hline C13-C14-H14A & 109.5 \\
\hline C13-C14-H14B & 109.5 \\
\hline C13-C14-C15 & $110.6(3)$ \\
\hline H14A-C14-H14B & 108.1 \\
\hline C15-C14-H14A & 109.5 \\
\hline
\end{tabular}




$\begin{array}{ll}\text { C15-C14-H14B } & 109.5 \\ \text { C14-C15-H15 } & 116.6 \\ \text { C16-C15-C14 } & 126.7(4) \\ \text { C16-C15-H15 } & 116.6 \\ \text { C15-C16-H16A } & 120.0 \\ \text { C15-C16-H16B } & 120.0 \\ \text { H16A-C16-H16B } & 120.0 \\ \text { H17A-C17-H17B } & 109.5 \\ \text { H17A-C17-H17C } & 109.5 \\ \text { H17B-C17-H17C } & 109.5 \\ \text { O2-C17-H17A } & 109.5 \\ \text { O2-C17-H17B } & 109.5 \\ \text { O2-C17-H17C } & 109.5 \\ \text { C5-C24-H24A } & 109.5 \\ \text { C5-C24-H24B } & 109.5 \\ \text { C5-C24-H24C } & 109.5 \\ \text { H24A-C24-H24B } & 109.5 \\ \text { H24A-C24-H24C } & 109.5 \\ \text { H24B-C24-H24C } & 109.5 \\ \text { C8-N1-H1A } & 116.8(18) \\ \text { C8-N1-H1B } & 121(2) \\ \text { H1A-N1-H1B } & 122(3) \\ \text { C1-O1-C5 } & 114.90(17) \\ \text { C1-O2-C17 } & 117.08(19) \\ \text { C10-O3-H3A } & 109.5 \\ \text { C9-O4-C6 } & 114.95(19) \\ & \end{array}$

Symmetry transformations used to generate equivalent atoms: 
Table 4. Anisotropic displacement parameters $\left(\AA^{2} \mathrm{x} 10^{3}\right)$ for $\mathbf{1 5}$. The anisotropic displacement factor exponent takes the form: $-2 \mathrm{p}^{2}\left[\mathrm{~h}^{2} \mathrm{a}^{* 2} \mathrm{U}^{11}+\ldots+2 \mathrm{~h} \mathrm{k} \mathrm{a}^{*} \mathrm{~b}^{*} \mathrm{U}^{12}\right]$

\begin{tabular}{|c|c|c|c|c|c|c|}
\hline & $\mathrm{U}^{11}$ & $\mathrm{U}^{22}$ & $\mathrm{U}^{33}$ & $\mathrm{U}^{23}$ & $\mathrm{U}^{13}$ & $\mathrm{U}^{12}$ \\
\hline $\mathrm{C} 1$ & $21(1)$ & $27(1)$ & $27(1)$ & $-5(1)$ & $1(1)$ & $2(1)$ \\
\hline $\mathrm{C} 2$ & $24(1)$ & $26(1)$ & $29(1)$ & $-1(1)$ & $-2(1)$ & $3(1)$ \\
\hline $\mathrm{C} 3$ & $44(1)$ & $29(1)$ & $31(1)$ & $6(1)$ & $-6(1)$ & $-2(1)$ \\
\hline $\mathrm{C} 4$ & $50(1)$ & $44(2)$ & $26(1)$ & $4(1)$ & $5(1)$ & $-3(1)$ \\
\hline $\mathrm{C} 5$ & $42(1)$ & $40(1)$ & $22(1)$ & $2(1)$ & $1(1)$ & $4(1)$ \\
\hline C6 & $28(1)$ & $25(1)$ & $31(1)$ & $-3(1)$ & $5(1)$ & $-3(1)$ \\
\hline C7 & $36(1)$ & $32(1)$ & $37(1)$ & $-6(1)$ & $14(1)$ & $-11(1)$ \\
\hline $\mathrm{C} 8$ & $24(1)$ & $26(1)$ & $29(1)$ & $8(1)$ & $0(1)$ & $1(1)$ \\
\hline C9 & $55(2)$ & $30(1)$ & $41(1)$ & $0(1)$ & $-2(1)$ & $12(1)$ \\
\hline $\mathrm{C} 10$ & $24(1)$ & $22(1)$ & $25(1)$ & $-1(1)$ & $1(1)$ & $-1(1)$ \\
\hline C11 & $26(1)$ & $30(1)$ & $32(1)$ & $-2(1)$ & $-2(1)$ & $5(1)$ \\
\hline C12 & $25(1)$ & $49(2)$ & $46(1)$ & $-6(1)$ & $5(1)$ & $0(1)$ \\
\hline C13 & $66(2)$ & $35(2)$ & $46(2)$ & $5(1)$ & $-22(1)$ & $5(1)$ \\
\hline C14 & $69(2)$ & $39(2)$ & $54(2)$ & $0(1)$ & $-17(2)$ & $4(2)$ \\
\hline C15 & $85(3)$ & $36(2)$ & $81(2)$ & $0(2)$ & $-26(2)$ & $14(2)$ \\
\hline $\mathrm{C} 16$ & $78(3)$ & $92(4)$ & $139(5)$ & $54(3)$ & $-39(3)$ & $-1(3)$ \\
\hline C17 & $36(1)$ & $48(2)$ & $46(1)$ & $-10(1)$ & $-10(1)$ & $-8(1)$ \\
\hline $\mathrm{C} 24$ & $64(2)$ & $56(2)$ & $33(1)$ & $-5(1)$ & $15(1)$ & $5(2)$ \\
\hline N1 & $28(1)$ & $29(1)$ & $44(1)$ & $3(1)$ & $10(1)$ & $-1(1)$ \\
\hline $\mathrm{O} 1$ & $32(1)$ & $28(1)$ & $23(1)$ & $-1(1)$ & $4(1)$ & $6(1)$ \\
\hline $\mathrm{O} 2$ & $26(1)$ & $34(1)$ & $46(1)$ & $-11(1)$ & $-9(1)$ & $3(1)$ \\
\hline $\mathrm{O} 3$ & $27(1)$ & $24(1)$ & $32(1)$ & $-4(1)$ & $0(1)$ & $1(1)$ \\
\hline $\mathrm{O} 4$ & $37(1)$ & $26(1)$ & $28(1)$ & $2(1)$ & $-1(1)$ & $1(1)$ \\
\hline O5 & $34(1)$ & $26(1)$ & $47(1)$ & $1(1)$ & $6(1)$ & $-6(1)$ \\
\hline
\end{tabular}


Table 5. Hydrogen coordinates $\left(\mathrm{x} 10^{4}\right)$ and isotropic displacement parameters $\left(\AA^{2} \times 10^{3}\right)$ for 15 .

\begin{tabular}{|c|c|c|c|c|}
\hline & $\mathrm{X}$ & $\mathrm{y}$ & z & $\mathrm{U}(\mathrm{eq})$ \\
\hline $\mathrm{H} 2$ & 7663 & 5358 & 8419 & 31 \\
\hline H3 & 5283 & 4244 & 8911 & 42 \\
\hline $\mathrm{H} 4 \mathrm{~A}$ & 3870 & 6297 & 9195 & 48 \\
\hline H4B & 4880 & 5630 & 9647 & 48 \\
\hline H5 & 7087 & 7450 & 9554 & 42 \\
\hline H6 & 7944 & 9540 & 8285 & 33 \\
\hline H7A & 8852 & 6863 & 7905 & 42 \\
\hline H7B & 7750 & 7720 & 7507 & 42 \\
\hline H9A & 4764 & 10195 & 8516 & 63 \\
\hline H9B & 3818 & 10493 & 8017 & 63 \\
\hline H9C & 5706 & 11175 & 8114 & 63 \\
\hline H10 & 5571 & 6361 & 7865 & 29 \\
\hline H11 & 3166 & 7120 & 8341 & 35 \\
\hline $\mathrm{H} 12 \mathrm{~A}$ & 2340 & 4080 & 8273 & 48 \\
\hline H12B & 1022 & 5459 & 8421 & 48 \\
\hline H13A & 8502 & 5161 & 9318 & 59 \\
\hline H13B & 7177 & 4120 & 9604 & 59 \\
\hline $\mathrm{H} 14 \mathrm{~A}$ & 8922 & 3279 & 8754 & 65 \\
\hline H14B & 7508 & 2240 & 9013 & 65 \\
\hline H15 & 9429 & 2058 & 9696 & 80 \\
\hline H16A & 11810 & 2618 & 9002 & 123 \\
\hline H16B & 12144 & 1884 & 9533 & 123 \\
\hline H17A & 10234 & 9258 & 8725 & 65 \\
\hline H17B & 10754 & 8357 & 9198 & 65 \\
\hline $\mathrm{H} 17 \mathrm{C}$ & 8967 & 9270 & 9177 & 65 \\
\hline H24A & 5521 & 9691 & 9713 & 76 \\
\hline H24B & 4783 & 8331 & 10027 & 76 \\
\hline $\mathrm{H} 24 \mathrm{C}$ & 3757 & 8872 & 9562 & 76 \\
\hline H3A & 4860 & 4042 & 7731 & 42 \\
\hline H1A & $10780(40)$ & $7090(40)$ & $7257(10)$ & $33(7)$ \\
\hline H1B & $12110(50)$ & $8490(40)$ & $7237(11)$ & $45(8)$ \\
\hline
\end{tabular}

Table 6. Torsion angles $\left[{ }^{\circ}\right]$ for $\mathbf{1 5}$. 
The X-ray structure of compound 18
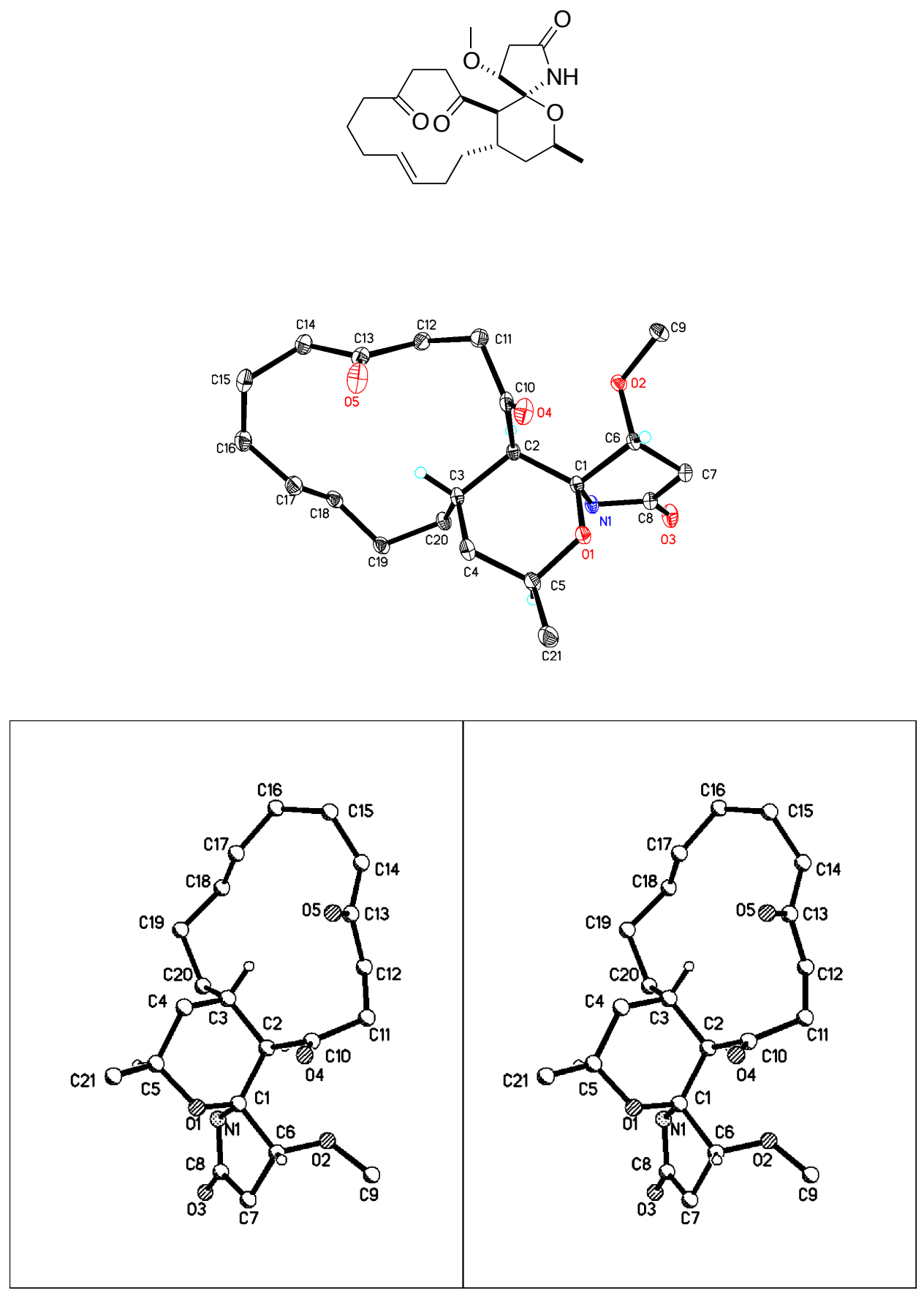
Table 1. Crystal data and structure refinement for $\mathbf{1 8 .}$

Identification code

Empirical formula

Formula weight

Temperature

Wavelength

Crystal system

Space group

Unit cell dimensions

Volume

Z

Density (calculated)

Absorption coefficient

$\mathrm{F}(000)$

Crystal size

Theta range for data collection

Index ranges

Reflections collected

Independent reflections

Completeness to theta $=26.000^{\circ}$

Absorption correction

Max. and min. transmission

Refinement method

Data / restraints / parameters

Goodness-of-fit on $\mathrm{F}^{2}$

Final $\mathrm{R}$ indices [I $>2 \operatorname{sigma}(\mathrm{I})]$

$\mathrm{R}$ indices (all data)

Absolute structure parameter

Extinction coefficient

Largest diff. peak and hole

\section{8}

$\mathrm{C}_{21} \mathrm{H}_{31} \mathrm{~N} \mathrm{O}_{5}$

377.47

$173.1500 \mathrm{~K}$

$0.71073 \AA$

Monoclinic

P 1211

$\mathrm{a}=11.5555(11) \AA \quad \mathrm{a}=90^{\circ}$.

$\mathrm{b}=6.7668(6) \AA \quad \mathrm{b}=98.846(5)^{\circ}$.

$\mathrm{c}=12.9264(14) \AA \quad \mathrm{g}=90^{\circ}$.

998.74(17) $\AA^{3}$

2

$1.255 \mathrm{Mg} / \mathrm{m}^{3}$

$0.089 \mathrm{~mm}^{-1}$

408

$0.65 \times 0.31 \times 0.28 \mathrm{~mm}^{3}$

3.407 to $27.472^{\circ}$.

$-14<=\mathrm{h}<=14,-8<=\mathrm{k}<=8,-16<=\mathrm{l}<=16$

11100

$4427[\mathrm{R}($ int $)=0.0228]$

$99.2 \%$

Semi-empirical from equivalents

1.0000 and 0.8738

Full-matrix least-squares on $\mathrm{F}^{2}$

4427 / 1 / 250

1.056

$\mathrm{R} 1=0.0308, \mathrm{wR} 2=0.0754$

$\mathrm{R} 1=0.0312, \mathrm{wR} 2=0.0757$

$-0.1(3)$

$\mathrm{n} / \mathrm{a}$

0.227 and -0.148 e. $\AA^{-3}$ 
Table 2. Atomic coordinates $\left(\mathrm{x}_{10} 0^{4}\right)$ and equivalent isotropic displacement parameters $\left(\AA^{2} \mathrm{x}\right.$ $10^{3}$ ) for $18 . \quad U(e q)$ is defined as one third of the trace of the orthogonalized $U^{i j}$ tensor.

\begin{tabular}{|c|c|c|c|c|}
\hline & $\mathrm{x}$ & $\mathrm{y}$ & $\mathrm{z}$ & $\mathrm{U}(\mathrm{eq})$ \\
\hline $\mathrm{O} 1$ & $8462(1)$ & $5696(2)$ & $7957(1)$ & $22(1)$ \\
\hline $\mathrm{O} 2$ & $7509(1)$ & 5993(2) & $5223(1)$ & $22(1)$ \\
\hline $\mathrm{O} 3$ & $9240(1)$ & $948(2)$ & $6117(1)$ & $34(1)$ \\
\hline $\mathrm{O} 4$ & $6816(1)$ & $9005(2)$ & $7027(1)$ & $31(1)$ \\
\hline $\mathrm{O} 5$ & $3926(1)$ & $9074(2)$ & $7449(1)$ & $40(1)$ \\
\hline N1 & $7958(1)$ & $2926(2)$ & $6844(1)$ & $21(1)$ \\
\hline $\mathrm{C} 1$ & $7768(1)$ & $5022(2)$ & $7024(1)$ & $19(1)$ \\
\hline $\mathrm{C} 2$ & $6454(1)$ & $5517(2)$ & $6962(1)$ & $18(1)$ \\
\hline $\mathrm{C} 3$ & $5960(1)$ & $5126(2)$ & $8002(1)$ & $21(1)$ \\
\hline $\mathrm{C} 4$ & $6834(1)$ & $5889(3)$ & $8928(1)$ & $25(1)$ \\
\hline $\mathrm{C} 5$ & $8068(2)$ & $5121(3)$ & $8916(1)$ & $25(1)$ \\
\hline C6 & $8358(1)$ & $5982(3)$ & $6153(1)$ & $20(1)$ \\
\hline $\mathrm{C} 7$ & $9353(1)$ & $4554(2)$ & $6045(1)$ & $22(1)$ \\
\hline $\mathrm{C} 8$ & $8876(1)$ & $2568(3)$ & $6321(1)$ & $23(1)$ \\
\hline C9 & $7821(2)$ & $7325(3)$ & $4459(1)$ & $29(1)$ \\
\hline $\mathrm{C} 10$ & $6197(1)$ & $7663(3)$ & $6649(1)$ & $23(1)$ \\
\hline $\mathrm{C} 11$ & $5138(2)$ & $8062(3)$ & $5819(1)$ & $28(1)$ \\
\hline $\mathrm{C} 12$ & $3986(2)$ & $7061(3)$ & $5955(1)$ & $26(1)$ \\
\hline $\mathrm{C} 13$ & $3403(1)$ & $7949(3)$ & $6816(2)$ & $26(1)$ \\
\hline C14 & $2135(1)$ & $7406(3)$ & $6820(1)$ & $28(1)$ \\
\hline C15 & $1696(2)$ & $7664(3)$ & $7865(2)$ & $33(1)$ \\
\hline $\mathrm{C} 16$ & $1853(2)$ & $5816(4)$ & $8548(2)$ & $36(1)$ \\
\hline $\mathrm{C} 17$ & $3098(2)$ & $5139(3)$ & $8861(1)$ & $31(1)$ \\
\hline C18 & $3542(2)$ & $3469(3)$ & $8566(1)$ & $27(1)$ \\
\hline C19 & $4763(1)$ & $2724(3)$ & $8933(1)$ & $26(1)$ \\
\hline $\mathrm{C} 20$ & $5606(2)$ & 2968(3) & $8126(1)$ & $24(1)$ \\
\hline $\mathrm{C} 21$ & $8942(2)$ & $6005(4)$ & $9788(1)$ & $37(1)$ \\
\hline
\end{tabular}


Table 3. Bond lengths $[\AA]$ and angles $\left[{ }^{\circ}\right]$ for $\mathbf{1 8 .}$

\begin{tabular}{|c|c|}
\hline $\mathrm{O} 1-\mathrm{C} 1$ & $1.4164(19)$ \\
\hline $\mathrm{O} 1-\mathrm{C} 5$ & $1.4392(19)$ \\
\hline $\mathrm{O} 2-\mathrm{C} 6$ & $1.4303(19)$ \\
\hline $\mathrm{O} 2-\mathrm{C} 9$ & $1.424(2)$ \\
\hline O3-C8 & $1.218(2)$ \\
\hline O4-C10 & $1.212(2)$ \\
\hline O5-C13 & $1.210(2)$ \\
\hline N1-C1 & $1.460(2)$ \\
\hline N1-C8 & $1.364(2)$ \\
\hline N1-H1 & $0.79(3)$ \\
\hline $\mathrm{C} 1-\mathrm{C} 2$ & $1.545(2)$ \\
\hline C1-C6 & $1.546(2)$ \\
\hline $\mathrm{C} 2-\mathrm{H} 2$ & 0.9800 \\
\hline C2-C3 & $1.562(2)$ \\
\hline C2-C10 & $1.524(2)$ \\
\hline C3-H3 & 0.9800 \\
\hline C3-C4 & $1.533(2)$ \\
\hline C3-C20 & $1.532(2)$ \\
\hline C4-H4A & 0.9700 \\
\hline C4-H4B & 0.9700 \\
\hline C4-C5 & $1.519(2)$ \\
\hline C5-H5 & 0.9800 \\
\hline C5-C21 & $1.516(2)$ \\
\hline C6-H6 & 0.9800 \\
\hline C6-C7 & $1.525(2)$ \\
\hline C7-H7A & 0.9700 \\
\hline C7-H7B & 0.9700 \\
\hline C7-C8 & $1.516(2)$ \\
\hline C9-H9A & 0.9600 \\
\hline C9-H9B & 0.9600 \\
\hline C9-H9C & 0.9600 \\
\hline C10-C11 & $1.522(2)$ \\
\hline C11-H11A & 0.9700 \\
\hline C11-H11B & 0.9700 \\
\hline C11-C12 & $1.528(2)$ \\
\hline $\mathrm{C} 12-\mathrm{H} 12 \mathrm{~A}$ & 0.9700 \\
\hline $\mathrm{C} 12-\mathrm{H} 12 \mathrm{~B}$ & 0.9700 \\
\hline C12-C13 & $1.512(2)$ \\
\hline C13-C14 & $1.511(2)$ \\
\hline C14-H14A & 0.9700 \\
\hline C14-H14B & 0.9700 \\
\hline C14-C15 & $1.525(2)$ \\
\hline
\end{tabular}




\begin{tabular}{|c|c|}
\hline C15-H15A & 0.9700 \\
\hline C15-H15B & 0.9700 \\
\hline $\mathrm{C} 15-\mathrm{C} 16$ & $1.525(3)$ \\
\hline C16-H16A & 0.9700 \\
\hline C16-H16B & 0.9700 \\
\hline C16-C17 & $1.505(2)$ \\
\hline C17-H17 & 0.9300 \\
\hline C17-C18 & $1.321(3)$ \\
\hline C18-H18 & 0.9300 \\
\hline C18-C19 & $1.504(2)$ \\
\hline C19-H19A & 0.9700 \\
\hline C19-H19B & 0.9700 \\
\hline C19-C20 & $1.541(2)$ \\
\hline C20-H20A & 0.9700 \\
\hline C20-H20B & 0.9700 \\
\hline $\mathrm{C} 21-\mathrm{H} 21 \mathrm{~A}$ & 0.9600 \\
\hline C21-H21B & 0.9600 \\
\hline $\mathrm{C} 21-\mathrm{H} 21 \mathrm{C}$ & 0.9600 \\
\hline $\mathrm{C} 1-\mathrm{O} 1-\mathrm{C} 5$ & $115.72(12)$ \\
\hline C9-O2-C6 & $111.84(12)$ \\
\hline C1-N1-H1 & $123.1(18)$ \\
\hline C8-N1-C1 & $113.54(13)$ \\
\hline C8-N1-H1 & $123.3(18)$ \\
\hline $\mathrm{O} 1-\mathrm{C} 1-\mathrm{N} 1$ & $111.67(13)$ \\
\hline $\mathrm{O} 1-\mathrm{C} 1-\mathrm{C} 2$ & $113.46(12)$ \\
\hline $\mathrm{O} 1-\mathrm{C} 1-\mathrm{C} 6$ & $103.28(12)$ \\
\hline $\mathrm{N} 1-\mathrm{C} 1-\mathrm{C} 2$ & $111.89(12)$ \\
\hline N1-C1-C6 & $101.41(12)$ \\
\hline $\mathrm{C} 2-\mathrm{C} 1-\mathrm{C} 6$ & $114.24(13)$ \\
\hline $\mathrm{C} 1-\mathrm{C} 2-\mathrm{H} 2$ & 107.7 \\
\hline $\mathrm{C} 1-\mathrm{C} 2-\mathrm{C} 3$ & $113.87(13)$ \\
\hline C3-C2-H2 & 107.7 \\
\hline $\mathrm{C} 10-\mathrm{C} 2-\mathrm{C} 1$ & $111.69(12)$ \\
\hline $\mathrm{C} 10-\mathrm{C} 2-\mathrm{H} 2$ & 107.7 \\
\hline $\mathrm{C} 10-\mathrm{C} 2-\mathrm{C} 3$ & $107.92(13)$ \\
\hline C2-C3-H3 & 107.0 \\
\hline $\mathrm{C} 4-\mathrm{C} 3-\mathrm{C} 2$ & $109.34(12)$ \\
\hline C4-C3-H3 & 107.0 \\
\hline $\mathrm{C} 20-\mathrm{C} 3-\mathrm{C} 2$ & $112.98(13)$ \\
\hline C20-C3-H3 & 107.0 \\
\hline C20-C3-C4 & $113.18(15)$ \\
\hline C3-C4-H4A & 109.1 \\
\hline C3-C4-H4B & 109.1 \\
\hline
\end{tabular}




\begin{tabular}{ll} 
H4A-C4-H4B & 107.8 \\
C5-C4-C3 & $112.60(13)$ \\
C5-C4-H4A & 109.1 \\
C5-C4-H4B & 109.1 \\
O1-C5-C4 & $109.72(14)$ \\
O1-C5-H5 & 109.7 \\
O1-C5-C21 & $105.74(14)$ \\
C4-C5-H5 & 109.7 \\
C21-C5-C4 & $112.12(15)$ \\
C21-C5-H5 & 109.7 \\
O2-C6-C1 & $107.00(12)$ \\
O2-C6-H6 & 111.9 \\
O2-C6-C7 & $110.82(13)$ \\
C1-C6-H6 & 111.9 \\
C7-C6-C1 & $102.91(13)$ \\
C7-C6-H6 & 111.9 \\
C6-C7-H7A & 111.1 \\
C6-C7-H7B & 111.1 \\
H7A-C7-H7B & 109.0 \\
C8-C7-C6 & $103.52(12)$ \\
C8-C7-H7A & 111.1 \\
C8-C7-H7B & 111.1 \\
O3-C8-N1 & $126.02(16)$ \\
O3-C8-C7 & $126.64(14)$ \\
N1-C8-C7 & $107.34(14)$ \\
O2-C9-H9A & 109.5 \\
O2-C9-H9B & 109.5 \\
O2-C9-H9C & 109.5 \\
H9A-C9-H9B & 109.5 \\
H9A-C9-H9C & 109.5 \\
H9B-C9-H9C & 109.5 \\
O4-C10-C2 & $121.91(15)$ \\
O4-C10-C11 & $120.79(16)$ \\
C11-C10-C2 & $117.29(14)$ \\
C10-C11-H11A & 108.0 \\
C10-C11-H11B & 108.0 \\
C10-C11-C12 & $117.39(14)$ \\
H11A-C11-H11B & 107.2 \\
C12-C11-H11A & 108.0 \\
C12-C11-H11B & 108.0 \\
C11-C12-H12A & 108.8 \\
H11-C12-H12B & 108.8 \\
C13-C12-C11 & 107.7 \\
& $113.81(15)$ \\
\hline
\end{tabular}




\begin{tabular}{|c|c|}
\hline C13-C12-H12A & 108.8 \\
\hline C13-C12-H12B & 108.8 \\
\hline $\mathrm{O} 5-\mathrm{C} 13-\mathrm{C} 12$ & $121.01(16)$ \\
\hline O5-C13-C14 & $122.35(17)$ \\
\hline C14-C13-C12 & $116.61(15)$ \\
\hline C13-C14-H14A & 108.4 \\
\hline C13-C14-H14B & 108.4 \\
\hline C13-C14-C15 & $115.43(15)$ \\
\hline H14A-C14-H14B & 107.5 \\
\hline C15-C14-H14A & 108.4 \\
\hline C15-C14-H14B & 108.4 \\
\hline C14-C15-H15A & 109.0 \\
\hline C14-C15-H15B & 109.0 \\
\hline C14-C15-C16 & $113.13(16)$ \\
\hline H15A-C15-H15B & 107.8 \\
\hline C16-C15-H15A & 109.0 \\
\hline C16-C15-H15B & 109.0 \\
\hline C15-C16-H16A & 108.4 \\
\hline C15-C16-H16B & 108.4 \\
\hline H16A-C16-H16B & 107.5 \\
\hline C17-C16-C15 & $115.40(16)$ \\
\hline C17-C16-H16A & 108.4 \\
\hline C17-C16-H16B & 108.4 \\
\hline C16-C17-H17 & 117.3 \\
\hline C18-C17-C16 & $125.49(18)$ \\
\hline C18-C17-H17 & 117.3 \\
\hline C17-C18-H18 & 117.2 \\
\hline C17-C18-C19 & $125.67(17)$ \\
\hline C19-C18-H18 & 117.2 \\
\hline C18-C19-H19A & 108.7 \\
\hline C18-C19-H19B & 108.7 \\
\hline C18-C19-C20 & $114.24(14)$ \\
\hline H19A-C19-H19B & 107.6 \\
\hline C20-C19-H19A & 108.7 \\
\hline C20-C19-H19B & 108.7 \\
\hline C3-C20-C19 & $112.32(14)$ \\
\hline C3-C20-H20A & 109.1 \\
\hline C3-C20-H20B & 109.1 \\
\hline C19-C20-H20A & 109.1 \\
\hline C19-C20-H20B & 109.1 \\
\hline H20A-C20-H20B & 107.9 \\
\hline C5-C21-H21A & 109.5 \\
\hline C5-C21-H21B & 109.5 \\
\hline $\mathrm{C} 5-\mathrm{C} 21-\mathrm{H} 21 \mathrm{C}$ & 109.5 \\
\hline
\end{tabular}


H21A-C21-H21B $\quad 109.5$

H21A-C21-H21C $\quad 109.5$

H21B-C21-H21C $\quad 109.5$

Symmetry transformations used to generate equivalent atoms: 
Table 4. Anisotropic displacement parameters $\left(\AA^{2} \mathrm{x} 10^{3}\right)$ for $\mathbf{1 8}$. The anisotropic displacement factor exponent takes the form: $-2 \mathrm{p}^{2}\left[\mathrm{~h}^{2} \mathrm{a}^{* 2} \mathrm{U}^{11}+\ldots+2 \mathrm{~h} \mathrm{k} \mathrm{a}^{*} \mathrm{~b}^{*} \mathrm{U}^{12}\right]$

\begin{tabular}{|c|c|c|c|c|c|c|}
\hline & $\mathrm{U}^{11}$ & $\mathrm{U}^{22}$ & $\mathrm{U}^{33}$ & $\mathrm{U}^{23}$ & $\mathrm{U}^{13}$ & $\mathrm{U}^{12}$ \\
\hline $\mathrm{O} 1$ & $21(1)$ & $26(1)$ & $20(1)$ & $-2(1)$ & $6(1)$ & $-5(1)$ \\
\hline $\mathrm{O} 2$ & $22(1)$ & $23(1)$ & $22(1)$ & $3(1)$ & $5(1)$ & $-4(1)$ \\
\hline $\mathrm{O} 3$ & $36(1)$ & $22(1)$ & $49(1)$ & $-4(1)$ & $21(1)$ & $3(1)$ \\
\hline $\mathrm{O} 4$ & $28(1)$ & $17(1)$ & $50(1)$ & $-2(1)$ & $14(1)$ & $-4(1)$ \\
\hline $\mathrm{O} 5$ & $31(1)$ & $34(1)$ & $57(1)$ & $-19(1)$ & $12(1)$ & $-9(1)$ \\
\hline N1 & $22(1)$ & $16(1)$ & $27(1)$ & $1(1)$ & $10(1)$ & $-2(1)$ \\
\hline $\mathrm{C} 1$ & $19(1)$ & $18(1)$ & $22(1)$ & $-1(1)$ & $7(1)$ & $-2(1)$ \\
\hline $\mathrm{C} 2$ & $18(1)$ & $16(1)$ & $22(1)$ & $-1(1)$ & $7(1)$ & $-3(1)$ \\
\hline $\mathrm{C} 3$ & $20(1)$ & $20(1)$ & $25(1)$ & $-2(1)$ & $10(1)$ & $-3(1)$ \\
\hline $\mathrm{C} 4$ & $26(1)$ & $27(1)$ & $25(1)$ & $-6(1)$ & $12(1)$ & $-6(1)$ \\
\hline $\mathrm{C} 5$ & $26(1)$ & $29(1)$ & $22(1)$ & $-1(1)$ & $8(1)$ & $-4(1)$ \\
\hline C6 & $20(1)$ & $19(1)$ & $21(1)$ & $-1(1)$ & $7(1)$ & $-4(1)$ \\
\hline $\mathrm{C} 7$ & $19(1)$ & $23(1)$ & $25(1)$ & $-1(1)$ & $7(1)$ & $-2(1)$ \\
\hline $\mathrm{C} 8$ & $22(1)$ & $22(1)$ & $25(1)$ & $-2(1)$ & $6(1)$ & $-2(1)$ \\
\hline C9 & $34(1)$ & $27(1)$ & $27(1)$ & $6(1)$ & $8(1)$ & $-4(1)$ \\
\hline $\mathrm{C} 10$ & $20(1)$ & $21(1)$ & $31(1)$ & $1(1)$ & $12(1)$ & $0(1)$ \\
\hline C11 & $27(1)$ & $24(1)$ & $35(1)$ & $7(1)$ & $9(1)$ & $3(1)$ \\
\hline C12 & $23(1)$ & $25(1)$ & $30(1)$ & $2(1)$ & $4(1)$ & $1(1)$ \\
\hline C13 & $23(1)$ & $21(1)$ & $35(1)$ & $2(1)$ & $5(1)$ & $0(1)$ \\
\hline C14 & $22(1)$ & $31(1)$ & $33(1)$ & $-1(1)$ & $5(1)$ & $-2(1)$ \\
\hline C15 & $23(1)$ & $38(1)$ & $38(1)$ & $-8(1)$ & $8(1)$ & $3(1)$ \\
\hline C16 & $23(1)$ & $52(1)$ & $35(1)$ & $1(1)$ & $9(1)$ & $0(1)$ \\
\hline C17 & $23(1)$ & $45(1)$ & $25(1)$ & $-2(1)$ & $4(1)$ & $-5(1)$ \\
\hline C18 & $24(1)$ & $36(1)$ & $22(1)$ & $3(1)$ & $6(1)$ & $-9(1)$ \\
\hline C19 & $26(1)$ & $29(1)$ & $24(1)$ & $2(1)$ & $7(1)$ & $-6(1)$ \\
\hline $\mathrm{C} 20$ & $26(1)$ & $21(1)$ & $26(1)$ & $0(1)$ & $10(1)$ & $-4(1)$ \\
\hline $\mathrm{C} 21$ & $30(1)$ & $58(1)$ & $24(1)$ & $-4(1)$ & $5(1)$ & $-9(1)$ \\
\hline
\end{tabular}


Table 5. Hydrogen coordinates $\left(\mathrm{x} 10^{4}\right)$ and isotropic displacement parameters $\left(\AA^{2} \times 10^{3}\right)$ for 18 .

\begin{tabular}{|c|c|c|c|c|}
\hline & $\mathrm{x}$ & $\mathrm{y}$ & $\mathrm{z}$ & $\mathrm{U}(\mathrm{eq})$ \\
\hline $\mathrm{H} 2$ & 6018 & 4678 & 6418 & 22 \\
\hline H3 & 5247 & 5923 & 7975 & 25 \\
\hline $\mathrm{H} 4 \mathrm{~A}$ & 6845 & 7322 & 8910 & 30 \\
\hline H4B & 6576 & 5489 & 9576 & 30 \\
\hline H5 & 8076 & 3677 & 8977 & 30 \\
\hline H6 & 8648 & 7314 & 6346 & 23 \\
\hline H7A & 9530 & 4552 & 5335 & 26 \\
\hline H7B & 10055 & 4895 & 6524 & 26 \\
\hline H9A & 7204 & 7368 & 3870 & 43 \\
\hline H9B & 8531 & 6885 & 4234 & 43 \\
\hline H9C & 7937 & 8622 & 4759 & 43 \\
\hline H11A & 5335 & 7663 & 5146 & 33 \\
\hline H11B & 5006 & 9478 & 5788 & 33 \\
\hline H12A & 3452 & 7148 & 5300 & 31 \\
\hline H12B & 4135 & 5672 & 6105 & 31 \\
\hline $\mathrm{H} 14 \mathrm{~A}$ & 2025 & 6036 & 6605 & 34 \\
\hline H14B & 1653 & 8207 & 6300 & 34 \\
\hline $\mathrm{H} 15 \mathrm{~A}$ & 872 & 8011 & 7735 & 39 \\
\hline H15B & 2116 & 8751 & 8243 & 39 \\
\hline H16A & 1517 & 6066 & 9178 & 44 \\
\hline H16B & 1410 & 4749 & 8174 & 44 \\
\hline H17 & 3596 & 5964 & 9300 & 37 \\
\hline H18 & 3058 & 2687 & 8090 & 32 \\
\hline H19A & 4721 & 1334 & 9107 & 31 \\
\hline H19B & 5084 & 3423 & 9567 & 31 \\
\hline $\mathrm{H} 20 \mathrm{~A}$ & 6304 & 2185 & 8345 & 28 \\
\hline H20B & 5232 & 2468 & 7454 & 28 \\
\hline $\mathrm{H} 21 \mathrm{~A}$ & 9703 & 5457 & 9765 & 56 \\
\hline $\mathrm{H} 21 \mathrm{~B}$ & 8707 & 5706 & 10452 & 56 \\
\hline $\mathrm{H} 21 \mathrm{C}$ & 8968 & 7412 & 9700 & 56 \\
\hline $\mathrm{H} 1$ & $7590(20)$ & $2080(40)$ & $7058(18)$ & $32(6)$ \\
\hline
\end{tabular}


Table 6. Torsion angles $\left[{ }^{\circ}\right]$ for $\mathbf{1 8}$.

\begin{tabular}{lc}
\hline O1-C1-C2-C3 & $-43.80(18)$ \\
O1-C1-C2-C10 & $78.75(17)$ \\
O1-C1-C6-O2 & $-159.55(12)$ \\
O1-C1-C6-C7 & $83.62(14)$ \\
O2-C6-C7-C8 & $-83.19(15)$ \\
O4-C10-C11-C12 & $-133.12(17)$ \\
O5-C13-C14-C15 & $-23.1(3)$ \\
N1-C1-C2-C3 & $83.65(16)$ \\
N1-C1-C2-C10 & $-153.80(13)$ \\
N1-C1-C6-O2 & $84.69(14)$ \\
N1-C1-C6-C7 & $-32.14(15)$ \\
C1-O1-C5-C4 & $-58.96(18)$ \\
C1-O1-C5-C21 & $179.94(15)$ \\
C1-N1-C8-O3 & $176.70(16)$ \\
C1-N1-C8-C7 & $-3.32(19)$ \\
C1-C2-C3-C4 & $43.40(18)$ \\
C1-C2-C3-C20 & $-83.61(16)$ \\
C1-C2-C10-O4 & $-43.8(2)$ \\
C1-C2-C10-C11 & $134.88(14)$ \\
C1-C6-C7-C8 & $30.89(16)$ \\
C2-C1-C6-O2 & $-35.84(18)$ \\
C2-C1-C6-C7 & $-152.67(13)$ \\
C2-C3-C4-C5 & $-51.25(19)$ \\
C2-C3-C20-C19 & $-160.91(14)$ \\
C2-C10-C11-C12 & $48.1(2)$ \\
C3-C2-C10-O4 & $82.05(18)$ \\
C3-C2-C10-C11 & $-99.23(16)$ \\
C3-C4-C5-O1 & $58.51(19)$ \\
C3-C4-C5-C21 & $175.68(16)$ \\
C4-C3-C20-C19 & $74.15(17)$ \\
C5-O1-C1-N1 & $-75.49(16)$ \\
C5-O1-C1-C2 & $52.08(18)$ \\
C5-O1-C1-C6 & $176.30(13)$ \\
C6-C1-C2-C3 & $-161.84(13)$ \\
C6-C1-C2-C10 & $-39.29(18)$ \\
C6-C7-C8-O3 & $161.90(17)$ \\
C6-C7-C8-N1 & $-18.07(17)$ \\
C8-N1-C1-O1 & $-86.62(16)$ \\
C8-N1-C1-C2 & $144.97(14)$ \\
C8-N1-C1-C6 & $22.79(17)$ \\
C9-O2-C6-C1 & $163.10(14)$ \\
C9-O2-C6-C7 & $-85.43(17)$ \\
- & \\
&
\end{tabular}


C10-C2-C3-C4

$-81.20(16)$

C10-C2-C3-C20

151.80(14)

C10-C11-C12-C13

73.3(2)

C11-C12-C13-O5

$-13.3(3)$

C11-C12-C13-C14

164.90(15)

C12-C13-C14-C15

$158.78(17)$

C13-C14-C15-C16

$-90.3(2)$

C14-C15-C16-C17

61.3(2)

C15-C16-C17-C18

$-114.2(2)$

C16-C17-C18-C19

$-175.78(17)$

C17-C18-C19-C20

$-103.9(2)$

C18-C19-C20-C3

72.31(19)

$\mathrm{C} 20-\mathrm{C} 3-\mathrm{C} 4-\mathrm{C} 5$

75.64(17)

Symmetry transformations used to generate equivalent atoms:

Table 7. Hydrogen bonds for $\mathbf{1 8}\left[\AA\right.$ and $\left.{ }^{\circ}\right]$.

\begin{tabular}{lcccc}
\hline D-H...A & $d(D-H)$ & $d(H \ldots A)$ & $d(D \ldots A)$ & $<($ DHA $)$ \\
\hline N1-H1...O4\#1 & $0.79(3)$ & $2.26(3)$ & $2.9888(19)$ & $153(2)$
\end{tabular}

Symmetry transformations used to generate equivalent atoms:

\#1 x,y-1,z 
The X-ray structure of compound 19
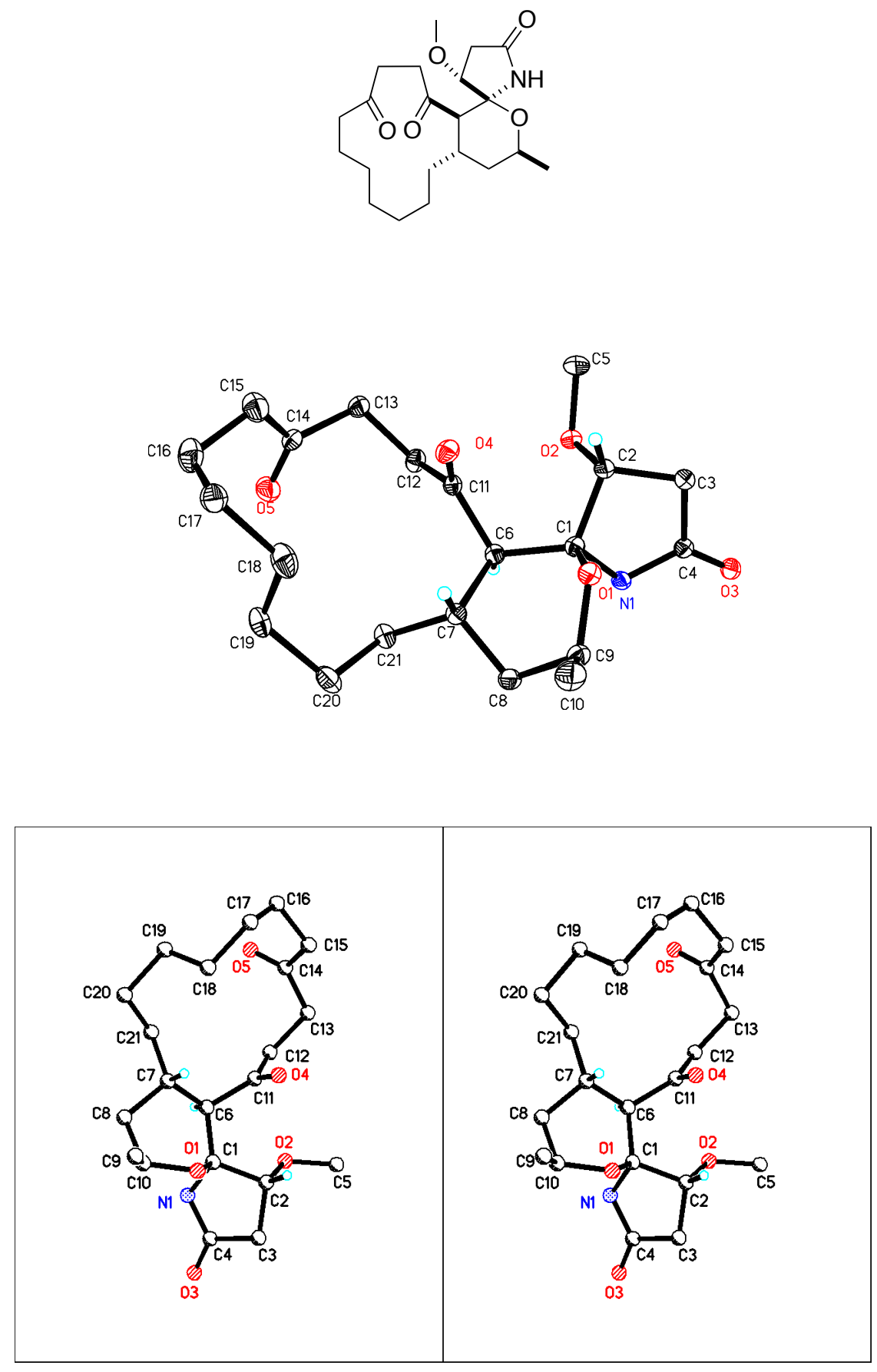
Table 1. Crystal data and structure refinement for 19.

Identification code

Empirical formula

Formula weight

Temperature

Wavelength

Crystal system

Space group

Unit cell dimensions

Volume

Z

Density (calculated)

Absorption coefficient

$\mathrm{F}(000)$

Crystal size

Theta range for data collection

Index ranges

Reflections collected

Independent reflections

Completeness to theta $=26.000^{\circ}$

Absorption correction

Max. and min. transmission

Refinement method

Data / restraints / parameters

Goodness-of-fit on $\mathrm{F}^{2}$

Final $\mathrm{R}$ indices [I $>2 \operatorname{sigma}(\mathrm{I})]$

$\mathrm{R}$ indices (all data)

Absolute structure parameter

Extinction coefficient

Largest diff. peak and hole
19

$\mathrm{C}_{21} \mathrm{H}_{33} \mathrm{~N} \mathrm{O}_{5}$

379.48

$173.1500 \mathrm{~K}$

$0.71073 \AA$

Orthorhombic

P 21212

$\begin{array}{ll}\mathrm{a}=11.4200(6) \AA & \mathrm{a}=90^{\circ} . \\ \mathrm{b}=19.1624(10) \AA & \mathrm{b}=90^{\circ} . \\ \mathrm{c}=9.3685(9) \AA & \mathrm{g}=90^{\circ} .\end{array}$

2050.2(2) $\AA^{3}$

4

$1.229 \mathrm{Mg} / \mathrm{m}^{3}$

$0.087 \mathrm{~mm}^{-1}$

824

$0.59 \times 0.44 \times 0.31 \mathrm{~mm}^{3}$

2.126 to $27.479^{\circ}$.

$-14<=\mathrm{h}<=14,-24<=\mathrm{k}<=24,-12<=\mathrm{l}<=12$

15775

$4653[\mathrm{R}($ int $)=0.0288]$

$99.7 \%$

Semi-empirical from equivalents

1.0000 and 0.8172

Full-matrix least-squares on $\mathrm{F}^{2}$

4653 / 8 / 269

1.113

$\mathrm{R} 1=0.0400, \mathrm{wR} 2=0.0987$

$\mathrm{R} 1=0.0407, \mathrm{wR} 2=0.0994$

$0.2(4)$

$\mathrm{n} / \mathrm{a}$

0.226 and -0.214 e..$\AA^{-3}$ 
Table 2. Atomic coordinates $\left(\mathrm{x}_{10} 0^{4}\right)$ and equivalent isotropic displacement parameters $\left(\AA^{2} \mathrm{x}\right.$ $10^{3}$ ) for $19 . \quad U(e q)$ is defined as one third of the trace of the orthogonalized $U^{i j}$ tensor.

\begin{tabular}{|c|c|c|c|c|}
\hline & $\mathrm{x}$ & $\mathrm{y}$ & $\mathrm{z}$ & $\mathrm{U}(\mathrm{eq})$ \\
\hline $\mathrm{O} 1$ & $2846(1)$ & $6318(1)$ & $5472(2)$ & $27(1)$ \\
\hline $\mathrm{O} 2$ & $1908(1)$ & $6062(1)$ & $9042(2)$ & $29(1)$ \\
\hline $\mathrm{O} 3$ & $3713(1)$ & $4444(1)$ & $7388(2)$ & $32(1)$ \\
\hline $\mathrm{O} 4$ & $1623(1)$ & $7496(1)$ & $7678(2)$ & $30(1)$ \\
\hline $\mathrm{O} 5$ & $3164(2)$ & $9032(1)$ & $9912(2)$ & $43(1)$ \\
\hline N1 & $3818(2)$ & $5640(1)$ & $7227(2)$ & $24(1)$ \\
\hline $\mathrm{C} 1$ & $3036(2)$ & $6229(1)$ & $6961(2)$ & $23(1)$ \\
\hline $\mathrm{C} 2$ & $1875(2)$ & $5943(1)$ & $7552(2)$ & $25(1)$ \\
\hline $\mathrm{C} 3$ & $1950(2)$ & $5163(1)$ & $7232(3)$ & $28(1)$ \\
\hline $\mathrm{C} 4$ & $3252(2)$ & $5022(1)$ & $7294(2)$ & $26(1)$ \\
\hline $\mathrm{C} 5$ & $783(2)$ & $6008(2)$ & $9685(3)$ & $50(1)$ \\
\hline C6 & $3470(2)$ & $6917(1)$ & $7651(2)$ & $23(1)$ \\
\hline $\mathrm{C} 7$ & $4013(2)$ & $7395(1)$ & $6491(2)$ & $24(1)$ \\
\hline $\mathrm{C} 8$ & $4726(2)$ & $6944(1)$ & $5463(2)$ & $29(1)$ \\
\hline C9 & $3899(2)$ & $6454(1)$ & $4657(2)$ & $30(1)$ \\
\hline $\mathrm{C} 10$ & $3477(3)$ & $6763(1)$ & $3258(3)$ & $41(1)$ \\
\hline C11 & $2480(2)$ & $7325(1)$ & $8364(2)$ & $24(1)$ \\
\hline $\mathrm{C} 12$ & $2633(2)$ & $7545(1)$ & $9904(2)$ & $30(1)$ \\
\hline C13 & $1797(2)$ & $8130(1)$ & $10347(2)$ & $32(1)$ \\
\hline C14 & $2180(2)$ & $8824(1)$ & $9712(2)$ & $30(1)$ \\
\hline $\mathrm{C} 15$ & $1311(2)$ & $9235(1)$ & $8876(3)$ & $44(1)$ \\
\hline C16 & $1821(3)$ & $9846(1)$ & $8021(3)$ & $50(1)$ \\
\hline $\mathrm{C} 17$ & $1928(4)$ & $9681(2)$ & $6503(5)$ & $40(1)$ \\
\hline C18 & $2813(2)$ & $9069(1)$ & $6110(3)$ & $46(1)$ \\
\hline C19 & $4039(4)$ & $9243(2)$ & $6440(5)$ & $36(1)$ \\
\hline $\mathrm{C} 20$ & $4959(2)$ & $8602(1)$ & $6114(3)$ & $41(1)$ \\
\hline $\mathrm{C} 21$ & $4719(2)$ & $7996(1)$ & $7135(3)$ & $30(1)$ \\
\hline C17A & $3050(6)$ & $9654(3)$ & $7093(7)$ & $50(2)$ \\
\hline C19A & $4029(5)$ & $8864(3)$ & $5235(6)$ & $42(2)$ \\
\hline
\end{tabular}


Table 3. Bond lengths $[\AA]$ and angles $\left[{ }^{\circ}\right]$ for 19.

\begin{tabular}{|c|c|}
\hline $\mathrm{O} 1-\mathrm{C} 1$ & $1.422(2)$ \\
\hline $\mathrm{O} 1-\mathrm{C} 9$ & $1.448(3)$ \\
\hline $\mathrm{O} 2-\mathrm{C} 2$ & $1.415(3)$ \\
\hline $\mathrm{O} 2-\mathrm{C} 5$ & $1.423(3)$ \\
\hline $\mathrm{O} 3-\mathrm{C} 4$ & $1.229(3)$ \\
\hline O4-C11 & $1.216(3)$ \\
\hline $\mathrm{O} 5-\mathrm{C} 14$ & $1.207(3)$ \\
\hline N1-C1 & $1.460(2)$ \\
\hline N1-C4 & $1.351(3)$ \\
\hline N1-H1 & $0.88(3)$ \\
\hline $\mathrm{C} 1-\mathrm{C} 2$ & $1.538(3)$ \\
\hline C1-C6 & $1.551(3)$ \\
\hline $\mathrm{C} 2-\mathrm{H} 2$ & 0.9800 \\
\hline C2-C3 & $1.527(3)$ \\
\hline C3-H3A & 0.9700 \\
\hline C3-H3B & 0.9700 \\
\hline C3-C4 & $1.512(3)$ \\
\hline C5-H5A & 0.9600 \\
\hline C5-H5B & 0.9600 \\
\hline C5-H5C & 0.9600 \\
\hline C6-H6 & 0.9800 \\
\hline C6-C7 & $1.551(3)$ \\
\hline C6-C11 & $1.528(3)$ \\
\hline C7-H7 & 0.9800 \\
\hline $\mathrm{C} 7-\mathrm{C} 8$ & $1.529(3)$ \\
\hline C7-C21 & $1.530(3)$ \\
\hline C8-H8A & 0.9700 \\
\hline C8-H8B & 0.9700 \\
\hline C8-C9 & $1.531(3)$ \\
\hline C9-H9 & 0.9800 \\
\hline C9-C10 & $1.517(3)$ \\
\hline C10-H10A & 0.9600 \\
\hline C10-H10B & 0.9600 \\
\hline C10-H10C & 0.9600 \\
\hline C11-C12 & $1.514(3)$ \\
\hline C12-H12A & 0.9700 \\
\hline C12-H12B & 0.9700 \\
\hline C12-C13 & $1.530(3)$ \\
\hline C13-H13A & 0.9700 \\
\hline C13-H13B & 0.9700 \\
\hline C13-C14 & $1.521(3)$ \\
\hline C14-C15 & $1.489(3)$ \\
\hline
\end{tabular}




\begin{tabular}{|c|c|}
\hline C15-H15A & 0.9700 \\
\hline C15-H15B & 0.9700 \\
\hline C15-C16 & $1.533(4)$ \\
\hline C16-H16A & 0.9700 \\
\hline C16-H16B & 0.9700 \\
\hline C16-H16C & 0.9700 \\
\hline C16-H16D & 0.9700 \\
\hline C16-C17 & $1.462(5)$ \\
\hline C16-C17A & $1.692(7)$ \\
\hline C17-H17A & 0.9700 \\
\hline C17-H17B & 0.9700 \\
\hline C17-C18 & $1.592(5)$ \\
\hline C18-H18A & 0.9700 \\
\hline C18-H18B & 0.9700 \\
\hline C18-H18C & 0.9700 \\
\hline C18-H18D & 0.9700 \\
\hline C18-C19 & $1.472(5)$ \\
\hline C18-C17A & $1.476(6)$ \\
\hline C18-C19A & $1.660(6)$ \\
\hline C19-H19A & 0.9700 \\
\hline C19-H19B & 0.9700 \\
\hline C19-C20 & $1.645(5)$ \\
\hline C20-H20A & 0.9700 \\
\hline C20-H20B & 0.9700 \\
\hline C20-H20C & 0.9700 \\
\hline C20-H20D & 0.9700 \\
\hline C20-C21 & $1.528(3)$ \\
\hline C20-C19A & $1.435(6)$ \\
\hline $\mathrm{C} 21-\mathrm{H} 21 \mathrm{~A}$ & 0.9700 \\
\hline C21-H21B & 0.9700 \\
\hline C17A-H17C & 0.9700 \\
\hline C17A-H17D & 0.9700 \\
\hline C19A-H19C & 0.9700 \\
\hline C19A-H19D & 0.9700 \\
\hline $\mathrm{C} 1-\mathrm{O} 1-\mathrm{C} 9$ & $114.35(16)$ \\
\hline $\mathrm{C} 2-\mathrm{O} 2-\mathrm{C} 5$ & $112.42(19)$ \\
\hline C1-N1-H1 & $125.9(19)$ \\
\hline C4-N1-C1 & $113.09(16)$ \\
\hline C4-N1-H1 & $120.7(19)$ \\
\hline O1-C1-N1 & $110.75(16)$ \\
\hline $\mathrm{O} 1-\mathrm{C} 1-\mathrm{C} 2$ & $105.36(15)$ \\
\hline $\mathrm{O} 1-\mathrm{C} 1-\mathrm{C} 6$ & $110.81(16)$ \\
\hline $\mathrm{N} 1-\mathrm{C} 1-\mathrm{C} 2$ & $101.01(15)$ \\
\hline
\end{tabular}




\begin{tabular}{|c|c|}
\hline N1-C1-C6 & $112.96(16)$ \\
\hline C2-C1-C6 & $115.36(17)$ \\
\hline $\mathrm{O} 2-\mathrm{C} 2-\mathrm{C} 1$ & $105.96(16)$ \\
\hline $\mathrm{O} 2-\mathrm{C} 2-\mathrm{H} 2$ & 112.2 \\
\hline $\mathrm{O} 2-\mathrm{C} 2-\mathrm{C} 3$ & $110.50(17)$ \\
\hline $\mathrm{C} 1-\mathrm{C} 2-\mathrm{H} 2$ & 112.2 \\
\hline C3-C2-C1 & $103.24(16)$ \\
\hline C3-C2-H2 & 112.2 \\
\hline $\mathrm{C} 2-\mathrm{C} 3-\mathrm{H} 3 \mathrm{~A}$ & 111.2 \\
\hline C2-C3-H3B & 111.2 \\
\hline $\mathrm{H} 3 \mathrm{~A}-\mathrm{C} 3-\mathrm{H} 3 \mathrm{~B}$ & 109.1 \\
\hline $\mathrm{C} 4-\mathrm{C} 3-\mathrm{C} 2$ & $102.85(16)$ \\
\hline C4-C3-H3A & 111.2 \\
\hline C4-C3-H3B & 111.2 \\
\hline O3-C4-N1 & $126.02(19)$ \\
\hline $\mathrm{O} 3-\mathrm{C} 4-\mathrm{C} 3$ & $125.76(19)$ \\
\hline N1-C4-C3 & $108.22(17)$ \\
\hline $\mathrm{O} 2-\mathrm{C} 5-\mathrm{H} 5 \mathrm{~A}$ & 109.5 \\
\hline O2-C5-H5B & 109.5 \\
\hline O2-C5-H5C & 109.5 \\
\hline H5A-C5-H5B & 109.5 \\
\hline $\mathrm{H} 5 \mathrm{~A}-\mathrm{C} 5-\mathrm{H} 5 \mathrm{C}$ & 109.5 \\
\hline H5B-C5-H5C & 109.5 \\
\hline C1-C6-H6 & 109.1 \\
\hline C7-C6-C1 & $109.74(17)$ \\
\hline C7-C6-H6 & 109.1 \\
\hline C11-C6-C1 & $112.35(16)$ \\
\hline C11-C6-H6 & 109.1 \\
\hline C11-C6-C7 & $107.48(16)$ \\
\hline C6-C7-H7 & 107.5 \\
\hline C8-C7-C6 & $108.69(17)$ \\
\hline C8-C7-H7 & 107.5 \\
\hline C8-C7-C21 & $113.19(18)$ \\
\hline C21-C7-C6 & $112.24(17)$ \\
\hline $\mathrm{C} 21-\mathrm{C} 7-\mathrm{H} 7$ & 107.5 \\
\hline C7-C8-H8A & 109.8 \\
\hline C7-C8-H8B & 109.8 \\
\hline C7-C8-C9 & $109.23(17)$ \\
\hline H8A-C8-H8B & 108.3 \\
\hline C9-C8-H8A & 109.8 \\
\hline C9-C8-H8B & 109.8 \\
\hline O1-C9-C8 & $111.22(17)$ \\
\hline O1-C9-H9 & 109.3 \\
\hline $\mathrm{O} 1-\mathrm{C} 9-\mathrm{C} 10$ & $105.15(19)$ \\
\hline
\end{tabular}




\begin{tabular}{|c|c|}
\hline C8-C9-H9 & 109.3 \\
\hline $\mathrm{C} 10-\mathrm{C} 9-\mathrm{C} 8$ & $112.50(19)$ \\
\hline C10-C9-H9 & 109.3 \\
\hline C9-C10-H10A & 109.5 \\
\hline C9-C10-H10B & 109.5 \\
\hline C9-C10-H10C & 109.5 \\
\hline H10A-C10-H10B & 109.5 \\
\hline H10A-C10-H10C & 109.5 \\
\hline H10B-C10-H10C & 109.5 \\
\hline O4-C11-C6 & $120.23(19)$ \\
\hline O4-C11-C12 & $121.41(19)$ \\
\hline C12-C11-C6 & $118.25(18)$ \\
\hline C11-C12-H12A & 109.0 \\
\hline C11-C12-H12B & 109.0 \\
\hline C11-C12-C13 & $113.02(19)$ \\
\hline H12A-C12-H12B & 107.8 \\
\hline C13-C12-H12A & 109.0 \\
\hline C13-C12-H12B & 109.0 \\
\hline C12-C13-H13A & 109.5 \\
\hline C12-C13-H13B & 109.5 \\
\hline H13A-C13-H13B & 108.1 \\
\hline C14-C13-C12 & $110.79(18)$ \\
\hline C14-C13-H13A & 109.5 \\
\hline C14-C13-H13B & 109.5 \\
\hline O5-C14-C13 & $119.7(2)$ \\
\hline O5-C14-C15 & $121.9(2)$ \\
\hline C15-C14-C13 & $118.5(2)$ \\
\hline C14-C15-H15A & 108.5 \\
\hline C14-C15-H15B & 108.5 \\
\hline C14-C15-C16 & $115.2(2)$ \\
\hline H15A-C15-H15B & 107.5 \\
\hline C16-C15-H15A & 108.5 \\
\hline C16-C15-H15B & 108.5 \\
\hline C15-C16-H16A & 109.2 \\
\hline C15-C16-H16B & 109.2 \\
\hline C15-C16-H16C & 108.6 \\
\hline C15-C16-H16D & 108.6 \\
\hline C15-C16-C17A & $114.7(3)$ \\
\hline H16A-C16-H16B & 107.9 \\
\hline H16C-C16-H16D & 107.6 \\
\hline C17-C16-C15 & $112.0(3)$ \\
\hline C17-C16-H16A & 109.2 \\
\hline C17-C16-H16B & 109.2 \\
\hline C17A-C16-H16C & 108.6 \\
\hline
\end{tabular}




\begin{tabular}{|c|c|}
\hline C17A-C16-H16D & 108.6 \\
\hline C16-C17-H17A & 108.3 \\
\hline C16-C17-H17B & 108.3 \\
\hline C16-C17-C18 & $115.9(3)$ \\
\hline H17A-C17-H17B & 107.4 \\
\hline C18-C17-H17A & 108.3 \\
\hline C18-C17-H17B & 108.3 \\
\hline C17-C18-H18A & 109.0 \\
\hline C17-C18-H18B & 109.0 \\
\hline H18A-C18-H18B & 107.8 \\
\hline H18C-C18-H18D & 108.2 \\
\hline C19-C18-C17 & $112.8(3)$ \\
\hline C19-C18-H18A & 109.0 \\
\hline C19-C18-H18B & 109.0 \\
\hline C17A-C18-H18C & 109.8 \\
\hline C17A-C18-H18D & 109.8 \\
\hline C17A-C18-C19A & $109.5(4)$ \\
\hline C19A-C18-H18C & 109.8 \\
\hline C19A-C18-H18D & 109.8 \\
\hline C18-C19-H19A & 108.9 \\
\hline C18-C19-H19B & 108.9 \\
\hline C18-C19-C20 & $113.5(3)$ \\
\hline H19A-C19-H19B & 107.7 \\
\hline C20-C19-H19A & 108.9 \\
\hline C20-C19-H19B & 108.9 \\
\hline C19-C20-H20A & 109.7 \\
\hline C19-C20-H20B & 109.7 \\
\hline H20A-C20-H20B & 108.2 \\
\hline H20C-C20-H20D & 107.0 \\
\hline C21-C20-C19 & $109.7(2)$ \\
\hline C21-C20-H20A & 109.7 \\
\hline C21-C20-H20B & 109.7 \\
\hline $\mathrm{C} 21-\mathrm{C} 20-\mathrm{H} 20 \mathrm{C}$ & 107.4 \\
\hline C21-C20-H20D & 107.4 \\
\hline C19A-C20-H20C & 107.4 \\
\hline C19A-C20-H20D & 107.4 \\
\hline C19A-C20-C21 & $119.5(3)$ \\
\hline C7-C21-H21A & 108.6 \\
\hline C7-C21-H21B & 108.6 \\
\hline C20-C21-C7 & $114.76(19)$ \\
\hline C20-C21-H21A & 108.6 \\
\hline C20-C21-H21B & 108.6 \\
\hline $\mathrm{H} 21 \mathrm{~A}-\mathrm{C} 21-\mathrm{H} 21 \mathrm{~B}$ & 107.6 \\
\hline C16-C17A-H17C & 109.8 \\
\hline
\end{tabular}




$\begin{array}{ll}\text { C16-C17A-H17D } & 109.8 \\ \text { C18-C17A-C16 } & 109.5(4) \\ \text { C18-C17A-H17C } & 109.8 \\ \text { C18-C17A-H17D } & 109.8 \\ \text { H17C-C17A-H17D } & 108.2 \\ \text { C18-C19A-H19C } & 108.6 \\ \text { C18-C19A-H19D } & 108.6 \\ \text { C20-C19A-C18 } & 114.7(4) \\ \text { C20-C19A-H19C } & 108.6 \\ \text { C20-C19A-H19D } & 108.6 \\ \text { H19C-C19A-H19D } & 107.6\end{array}$

Symmetry transformations used to generate equivalent atoms: 
Table 4. Anisotropic displacement parameters $\left(\AA^{2} \times 10^{3}\right)$ for $19 . \quad$ The anisotropic displacement factor exponent takes the form: $-2 \mathrm{p}^{2}\left[\mathrm{~h}^{2} \mathrm{a}^{* 2} \mathrm{U}^{11}+\ldots+2 \mathrm{hk} \mathrm{a}^{*} \mathrm{~b}^{*} \mathrm{U}^{12}\right]$

\begin{tabular}{lcccccc}
\hline & $\mathrm{U} 11$ & $\mathrm{U}^{22}$ & $\mathrm{U} 33$ & $\mathrm{U}^{23}$ & $\mathrm{U} 13$ & $\mathrm{U} 12$ \\
& & & & & & \\
\hline $\mathrm{O} 1$ & $28(1)$ & $30(1)$ & $23(1)$ & $0(1)$ & $-2(1)$ & $-1(1)$ \\
$\mathrm{O} 2$ & $27(1)$ & $29(1)$ & $30(1)$ & $-2(1)$ & $8(1)$ & $-1(1)$ \\
$\mathrm{O} 3$ & $30(1)$ & $22(1)$ & $44(1)$ & $1(1)$ & $5(1)$ & $2(1)$ \\
$\mathrm{O} 4$ & $28(1)$ & $30(1)$ & $33(1)$ & $0(1)$ & $-3(1)$ & $7(1)$ \\
$\mathrm{O} 5$ & $30(1)$ & $37(1)$ & $61(1)$ & $3(1)$ & $-4(1)$ & $-2(1)$ \\
$\mathrm{N} 1$ & $19(1)$ & $21(1)$ & $32(1)$ & $2(1)$ & $2(1)$ & $1(1)$ \\
$\mathrm{C} 1$ & $22(1)$ & $23(1)$ & $23(1)$ & $1(1)$ & $-1(1)$ & $2(1)$ \\
$\mathrm{C} 2$ & $21(1)$ & $25(1)$ & $29(1)$ & $0(1)$ & $1(1)$ & $1(1)$ \\
$\mathrm{C} 3$ & $25(1)$ & $25(1)$ & $35(1)$ & $-2(1)$ & $3(1)$ & $-2(1)$ \\
$\mathrm{C} 4$ & $25(1)$ & $23(1)$ & $28(1)$ & $0(1)$ & $3(1)$ & $1(1)$ \\
$\mathrm{C} 5$ & $40(1)$ & $49(2)$ & $61(2)$ & $-11(1)$ & $28(1)$ & $-9(1)$ \\
$\mathrm{C} 6$ & $22(1)$ & $22(1)$ & $25(1)$ & $1(1)$ & $-2(1)$ & $1(1)$ \\
$\mathrm{C} 7$ & $24(1)$ & $24(1)$ & $26(1)$ & $3(1)$ & $-2(1)$ & $2(1)$ \\
$\mathrm{C} 8$ & $28(1)$ & $28(1)$ & $31(1)$ & $2(1)$ & $5(1)$ & $1(1)$ \\
$\mathrm{C} 9$ & $34(1)$ & $29(1)$ & $26(1)$ & $-2(1)$ & $5(1)$ & $1(1)$ \\
$\mathrm{C} 10$ & $52(2)$ & $45(1)$ & $25(1)$ & $3(1)$ & $3(1)$ & $-1(1)$ \\
$\mathrm{C} 11$ & $26(1)$ & $19(1)$ & $27(1)$ & $2(1)$ & $1(1)$ & $1(1)$ \\
$\mathrm{C} 12$ & $39(1)$ & $26(1)$ & $25(1)$ & $0(1)$ & $1(1)$ & $2(1)$ \\
$\mathrm{C} 13$ & $36(1)$ & $30(1)$ & $31(1)$ & $-3(1)$ & $9(1)$ & $0(1)$ \\
$\mathrm{C} 14$ & $31(1)$ & $26(1)$ & $32(1)$ & $-6(1)$ & $5(1)$ & $2(1)$ \\
$\mathrm{C} 15$ & $40(1)$ & $38(1)$ & $54(2)$ & $6(1)$ & $-9(1)$ & $-3(1)$ \\
$\mathrm{C} 16$ & $60(2)$ & $32(1)$ & $58(2)$ & $8(1)$ & $-4(1)$ & $5(1)$ \\
$\mathrm{C} 17$ & $41(2)$ & $35(2)$ & $42(3)$ & $8(2)$ & $2(2)$ & $2(2)$ \\
$\mathrm{C} 18$ & $50(2)$ & $35(1)$ & $52(2)$ & $13(1)$ & $-14(1)$ & $-5(1)$ \\
$\mathrm{C} 19$ & $45(2)$ & $25(2)$ & $39(2)$ & $8(2)$ & $-4(2)$ & $-6(2)$ \\
$\mathrm{C} 20$ & $45(1)$ & $36(1)$ & $40(1)$ & $5(1)$ & $4(1)$ & $-14(1)$ \\
$\mathrm{C} 21$ & $31(1)$ & $27(1)$ & $33(1)$ & $2(1)$ & $-5(1)$ & $-3(1)$ \\
$\mathrm{C} 17 \mathrm{~A}$ & $59(4)$ & $35(3)$ & $56(4)$ & $7(3)$ & $5(3)$ & $-6(3)$ \\
$\mathrm{C} 19 \mathrm{~A}$ & $47(3)$ & $37(3)$ & $41(3)$ & $21(3)$ & $8(3)$ & $6(2)$ \\
& & & & & & \\
\hline
\end{tabular}


Table 5. Hydrogen coordinates $\left(\mathrm{x} 10^{4}\right)$ and isotropic displacement parameters $\left(\AA^{2} \times 10^{3}\right)$ for 19.

\begin{tabular}{|c|c|c|c|c|}
\hline & $\mathrm{x}$ & $\mathrm{y}$ & $\mathrm{z}$ & $\mathrm{U}(\mathrm{eq})$ \\
\hline $\mathrm{H} 2$ & 1193 & 6162 & 7101 & 30 \\
\hline $\mathrm{H} 3 \mathrm{~A}$ & 1533 & 4892 & 7944 & 34 \\
\hline H3B & 1635 & 5057 & 6295 & 34 \\
\hline $\mathrm{H} 5 \mathrm{~A}$ & 273 & 6356 & 9289 & 75 \\
\hline H5B & 851 & 6078 & 10696 & 75 \\
\hline $\mathrm{H} 5 \mathrm{C}$ & 465 & 5553 & 9503 & 75 \\
\hline H6 & 4069 & 6808 & 8367 & 28 \\
\hline H7 & 3366 & 7600 & 5946 & 29 \\
\hline H8A & 5298 & 6673 & 5992 & 35 \\
\hline H8B & 5141 & 7239 & 4791 & 35 \\
\hline H9 & 4302 & 6012 & 4468 & 36 \\
\hline $\mathrm{H} 10 \mathrm{~A}$ & 2939 & 6446 & 2811 & 61 \\
\hline H10B & 4134 & 6839 & 2638 & 61 \\
\hline $\mathrm{H} 10 \mathrm{C}$ & 3090 & 7199 & 3439 & 61 \\
\hline $\mathrm{H} 12 \mathrm{~A}$ & 3433 & 7701 & 10046 & 36 \\
\hline H12B & 2504 & 7144 & 10516 & 36 \\
\hline $\mathrm{H} 13 \mathrm{~A}$ & 1011 & 8021 & 10024 & 39 \\
\hline H13B & 1782 & 8166 & 11380 & 39 \\
\hline $\mathrm{H} 15 \mathrm{~A}$ & 726 & 9417 & 9528 & 53 \\
\hline H15B & 916 & 8922 & 8219 & 53 \\
\hline H16A & 1319 & 10251 & 8135 & 60 \\
\hline H16B & 2587 & 9963 & 8398 & 60 \\
\hline $\mathrm{H} 16 \mathrm{C}$ & 1993 & 10225 & 8675 & 60 \\
\hline H16D & 1230 & 10011 & 7358 & 60 \\
\hline H17A & 2174 & 10100 & 6004 & 48 \\
\hline H17B & 1159 & 9557 & 6144 & 48 \\
\hline H18A & 2594 & 8652 & 6633 & 55 \\
\hline H18B & 2747 & 8966 & 5099 & 55 \\
\hline $\mathrm{H} 18 \mathrm{C}$ & 2207 & 9201 & 5437 & 55 \\
\hline H18D & 2539 & 8668 & 6645 & 55 \\
\hline H19A & 4096 & 9370 & 7441 & 44 \\
\hline H19B & 4268 & 9647 & 5881 & 44 \\
\hline $\mathrm{H} 20 \mathrm{~A}$ & 4868 & 8445 & 5136 & 49 \\
\hline H20B & 5757 & 8764 & 6238 & 49 \\
\hline $\mathrm{H} 20 \mathrm{C}$ & 5250 & 8989 & 6680 & 49 \\
\hline $\mathrm{H} 20 \mathrm{D}$ & 5590 & 8460 & 5486 & 49 \\
\hline $\mathrm{H} 21 \mathrm{~A}$ & 4299 & 8175 & 7958 & 36 \\
\hline
\end{tabular}




\begin{tabular}{lllll} 
H21B & 5462 & 7815 & 7471 & 36 \\
H17C & 3301 & 10059 & 6555 & 60 \\
H17D & 3672 & 9527 & 7747 & 60 \\
H19C & 3842 & 8516 & 4520 & 50 \\
H19D & 4307 & 9276 & 4738 & 50 \\
H1 & $4590(30)$ & $5658(15)$ & $7250(30)$ & $44(8)$ \\
\hline
\end{tabular}


Table 6. Torsion angles $\left[{ }^{\circ}\right]$ for 19.

\begin{tabular}{|c|c|}
\hline $\mathrm{O} 1-\mathrm{C} 1-\mathrm{C} 2-\mathrm{O} 2$ & $-161.55(15)$ \\
\hline $\mathrm{O} 1-\mathrm{C} 1-\mathrm{C} 2-\mathrm{C} 3$ & $82.26(19)$ \\
\hline O1-C1-C6-C7 & $-21.6(2)$ \\
\hline O1-C1-C6-C11 & 97.91(19) \\
\hline $\mathrm{O} 2-\mathrm{C} 2-\mathrm{C} 3-\mathrm{C} 4$ & $-82.9(2)$ \\
\hline O4-C11-C12-C13 & $-14.5(3)$ \\
\hline O5-C14-C15-C16 & $13.0(4)$ \\
\hline $\mathrm{N} 1-\mathrm{C} 1-\mathrm{C} 2-\mathrm{O} 2$ & $83.13(18)$ \\
\hline $\mathrm{N} 1-\mathrm{C} 1-\mathrm{C} 2-\mathrm{C} 3$ & $-33.1(2)$ \\
\hline N1-C1-C6-C7 & $103.33(19)$ \\
\hline N1-C1-C6-C11 & $-137.17(18)$ \\
\hline C1-O1-C9-C8 & $-39.7(2)$ \\
\hline C1-O1-C9-C10 & $-161.78(18)$ \\
\hline C1-N1-C4-O3 & $173.6(2)$ \\
\hline C1-N1-C4-C3 & $-6.4(3)$ \\
\hline $\mathrm{C} 1-\mathrm{C} 2-\mathrm{C} 3-\mathrm{C} 4$ & $30.0(2)$ \\
\hline C1-C6-C7-C8 & $-40.1(2)$ \\
\hline C1-C6-C7-C21 & $-166.11(17)$ \\
\hline C1-C6-C11-O4 & $-56.6(2)$ \\
\hline C1-C6-C11-C12 & $127.13(19)$ \\
\hline C2-C1-C6-C7 & $-141.19(17)$ \\
\hline C2-C1-C6-C11 & $-21.7(2)$ \\
\hline C2-C3-C4-O3 & $164.3(2)$ \\
\hline C2-C3-C4-N1 & $-15.6(2)$ \\
\hline C4-N1-C1-O1 & $-85.9(2)$ \\
\hline C4-N1-C1-C2 & 25.3(2) \\
\hline C4-N1-C1-C6 & $149.12(18)$ \\
\hline $\mathrm{C} 5-\mathrm{O} 2-\mathrm{C} 2-\mathrm{C} 1$ & $163.58(19)$ \\
\hline $\mathrm{C} 5-\mathrm{O} 2-\mathrm{C} 2-\mathrm{C} 3$ & $-85.2(2)$ \\
\hline $\mathrm{C} 6-\mathrm{C} 1-\mathrm{C} 2-\mathrm{O} 2$ & $-39.0(2)$ \\
\hline C6-C1-C2-C3 & $-155.20(17)$ \\
\hline C6-C7-C8-C9 & $65.7(2)$ \\
\hline C6-C7-C21-C20 & $-162.16(19)$ \\
\hline C6-C11-C12-C13 & $161.75(17)$ \\
\hline C7-C6-C11-O4 & $64.2(2)$ \\
\hline C7-C6-C11-C12 & $-112.1(2)$ \\
\hline C7-C8-C9-O1 & $-25.8(2)$ \\
\hline C7-C8-C9-C10 & $91.8(2)$ \\
\hline C8-C7-C21-C20 & $74.3(2)$ \\
\hline C9-O1-C1-N1 & $-60.0(2)$ \\
\hline C9-O1-C1-C2 & $-168.44(16)$ \\
\hline C9-O1-C1-C6 & 66.1(2) \\
\hline
\end{tabular}




$\begin{array}{lc}\text { C11-C6-C7-C8 } & -162.57(17) \\ \text { C11-C6-C7-C21 } & 71.5(2) \\ \text { C11-C12-C13-C14 } & -74.2(2) \\ \text { C12-C13-C14-O5 } & -54.3(3) \\ \text { C12-C13-C14-C15 } & 126.7(2) \\ \text { C13-C14-C15-C16 } & -168.0(2) \\ \text { C14-C15-C16-C17 } & 102.5(3) \\ \text { C14-C15-C16-C17A } & 45.6(4) \\ \text { C15-C16-C17-C18 } & -64.0(4) \\ \text { C15-C16-C17A-C18 } & 57.3(5) \\ \text { C16-C17-C18-C19 } & -65.5(5) \\ \text { C17-C18-C19-C20 } & 177.2(3) \\ \text { C18-C19-C20-C21 } & -66.5(4) \\ \text { C19-C20-C21-C7 } & 100.7(3) \\ \text { C21-C7-C8-C9 } & -168.91(18) \\ \text { C21-C20-C19A-C18 } & 52.1(5) \\ \text { C17A-C18-C19A-C20 } & 64.6(5) \\ \text { C19A-C18-C17A-C16 } & -178.3(3) \\ \text { C19A-C20-C21-C7 } & 45.0(4)\end{array}$

Symmetry transformations used to generate equivalent atoms:

Table 7. Hydrogen bonds for $\mathbf{1 9}\left[\AA\right.$ and $\left.{ }^{\circ}\right]$.

\begin{tabular}{lcccc}
\hline D-H...A & $d(D-H)$ & $d(H \ldots A)$ & $d(D \ldots A)$ & $<($ DHA $)$ \\
\hline N1-H1...O3\#1 & $0.88(3)$ & $1.95(3)$ & $2.828(2)$ & $172(3)$
\end{tabular}

Symmetry transformations used to generate equivalent atoms:

$\# 1-\mathrm{x}+1,-\mathrm{y}+1, \mathrm{z}$ 
The X-ray structure of compound 1
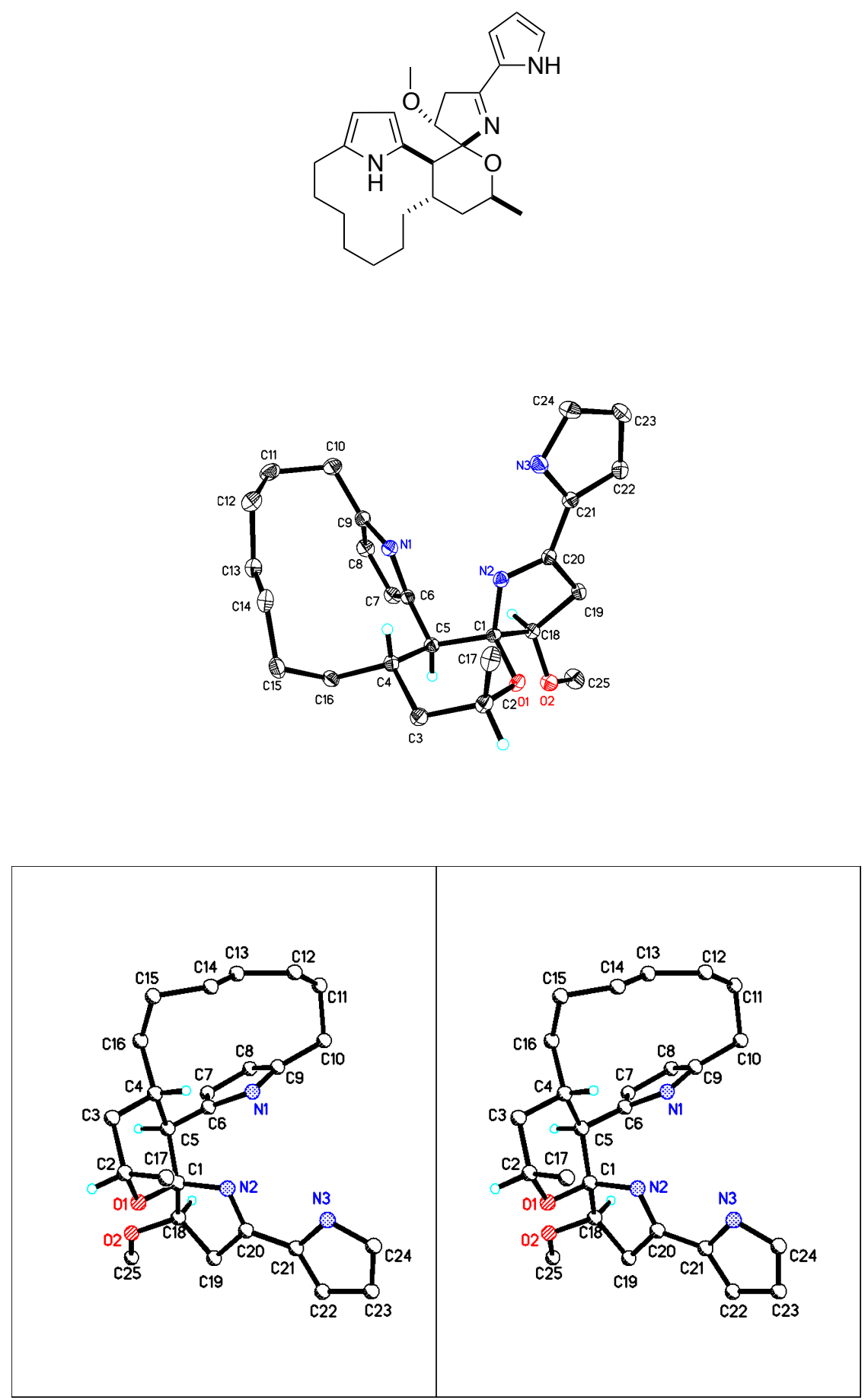
Table 1. Crystal data and structure refinement for $\mathbf{1}$.

Identification code

Empirical formula

Formula weight

Temperature

Wavelength

Crystal system

Space group

Unit cell dimensions

Volume

Z

Density (calculated)

Absorption coefficient

$\mathrm{F}(000)$

Crystal size

Theta range for data collection

Index ranges

Reflections collected

Independent reflections

Completeness to theta $=26.000^{\circ}$

Absorption correction

Max. and min. transmission

Refinement method

Data / restraints / parameters

Goodness-of-fit on $\mathrm{F}^{2}$

Final $\mathrm{R}$ indices [I $>2 \operatorname{sigma}(\mathrm{I})]$

$\mathrm{R}$ indices (all data)

Absolute structure parameter

Extinction coefficient

Largest diff. peak and hole
1

$\mathrm{C}_{25} \mathrm{H}_{37} \mathrm{~N}_{3} \mathrm{O}_{3}$

427.57

$173.1500 \mathrm{~K}$

$0.71073 \AA$

Orthorhombic

P 212121

$\mathrm{a}=8.174(3) \AA \quad \mathrm{a}=90^{\circ}$.

$\mathrm{b}=12.797(5) \AA \quad \mathrm{b}=90^{\circ}$.

$\mathrm{c}=21.823(8) \AA \quad \mathrm{g}=90^{\circ}$.

2282.7(15) $\AA^{3}$

4

$1.244 \mathrm{Mg} / \mathrm{m}^{3}$

$0.082 \mathrm{~mm}^{-1}$

928

$0.45 \times 0.13 \times 0.12 \mathrm{~mm}^{3}$

2.957 to $27.482^{\circ}$.

$-10<=\mathrm{h}<=10,-16<=\mathrm{k}<=16,-28<=1<=27$

14336

$5203[\mathrm{R}($ int $)=0.0457]$

$99.8 \%$

Semi-empirical from equivalents

1.0000 and 0.8596

Full-matrix least-squares on $\mathrm{F}^{2}$

5203 / 0 / 282

1.133

$\mathrm{R} 1=0.0595, \mathrm{wR} 2=0.1051$

$\mathrm{R} 1=0.0677, \mathrm{wR} 2=0.1086$

$-0.5(8)$

$\mathrm{n} / \mathrm{a}$

0.165 and -0.169 e. $\AA^{-3}$ 
Table 2. Atomic coordinates $\left(\mathrm{x}_{10} 0^{4}\right)$ and equivalent isotropic displacement parameters $\left(\AA^{2} \mathrm{x}\right.$ $10^{3}$ ) for $1 . \quad U(e q)$ is defined as one third of the trace of the orthogonalized $U^{i j}$ tensor.

\begin{tabular}{|c|c|c|c|c|}
\hline & $\mathrm{x}$ & $\mathrm{y}$ & $\mathrm{z}$ & $\mathrm{U}(\mathrm{eq})$ \\
\hline $\mathrm{O} 1$ & $5708(2)$ & $6120(2)$ & $6907(1)$ & $24(1)$ \\
\hline $\mathrm{O} 2$ & $7181(3)$ & $6824(2)$ & $5952(1)$ & $29(1)$ \\
\hline N1 & $1508(3)$ & $5071(2)$ & $5698(1)$ & $24(1)$ \\
\hline $\mathrm{N} 2$ & $3086(3)$ & $6625(2)$ & $6539(1)$ & $22(1)$ \\
\hline N3 & $386(3)$ & $7951(2)$ & $6773(1)$ & $28(1)$ \\
\hline $\mathrm{C} 1$ & $4654(3)$ & $6138(2)$ & $6380(1)$ & $20(1)$ \\
\hline $\mathrm{C} 2$ & $5313(4)$ & $5399(2)$ & $7398(1)$ & $26(1)$ \\
\hline $\mathrm{C} 3$ & $5158(4)$ & $4301(2)$ & $7143(1)$ & $26(1)$ \\
\hline $\mathrm{C} 4$ & $3933(4)$ & $4234(2)$ & $6615(1)$ & $23(1)$ \\
\hline $\mathrm{C} 5$ & $4397(4)$ & $5038(2)$ & $6113(1)$ & $21(1)$ \\
\hline C6 & $3170(4)$ & $5012(2)$ & $5599(1)$ & $23(1)$ \\
\hline $\mathrm{C} 7$ & $3388(4)$ & $4770(2)$ & 4994(1) & $30(1)$ \\
\hline $\mathrm{C} 8$ & $1817(4)$ & $4668(3)$ & $4723(1)$ & $33(1)$ \\
\hline C9 & $671(4)$ & $4847(2)$ & $5166(1)$ & $28(1)$ \\
\hline $\mathrm{C} 10$ & $-1147(4)$ & $4726(3)$ & $5153(2)$ & $35(1)$ \\
\hline C11 & $-1785(4)$ & $3610(3)$ & $5238(2)$ & $40(1)$ \\
\hline C12 & $-1461(4)$ & $3140(3)$ & $5867(2)$ & $40(1)$ \\
\hline C13 & 247(4) & $2669(2)$ & $5948(2)$ & $33(1)$ \\
\hline $\mathrm{C} 14$ & $842(4)$ & $2713(2)$ & $6609(2)$ & $33(1)$ \\
\hline $\mathrm{C} 15$ & $2628(4)$ & $2407(2)$ & $6692(2)$ & $33(1)$ \\
\hline $\mathrm{C} 16$ & $3840(4)$ & $3119(2)$ & $6359(1)$ & $27(1)$ \\
\hline $\mathrm{C} 17$ & $3859(4)$ & $5749(3)$ & $7779(1)$ & $33(1)$ \\
\hline C18 & $5467(4)$ & $6908(2)$ & $5932(1)$ & $23(1)$ \\
\hline C19 & $4762(4)$ & $7962(2)$ & $6127(1)$ & $25(1)$ \\
\hline $\mathrm{C} 20$ & $3176(4)$ & $7607(2)$ & $6410(1)$ & $22(1)$ \\
\hline $\mathrm{C} 21$ & $1828(4)$ & $8309(2)$ & $6530(1)$ & $24(1)$ \\
\hline $\mathrm{C} 22$ & $1667(4)$ & $9369(2)$ & $6449(1)$ & $29(1)$ \\
\hline $\mathrm{C} 23$ & 93(4) & $9661(2)$ & $6652(1)$ & $32(1)$ \\
\hline $\mathrm{C} 24$ & $-657(4)$ & $8763(3)$ & $6846(2)$ & $34(1)$ \\
\hline $\mathrm{C} 25$ & $7964(4)$ & $7411(3)$ & $5479(2)$ & $39(1)$ \\
\hline $\mathrm{O} 3$ & $9260(3)$ & $5773(2)$ & $6848(1)$ & $38(1)$ \\
\hline
\end{tabular}


Table 3. Bond lengths $[\AA]$ and angles $\left[{ }^{\circ}\right]$ for $\mathbf{1}$.

\begin{tabular}{|c|c|}
\hline $\mathrm{O} 1-\mathrm{C} 1$ & $1.437(3)$ \\
\hline $\mathrm{O} 1-\mathrm{C} 2$ & $1.451(3)$ \\
\hline $\mathrm{O} 2-\mathrm{C} 18$ & $1.405(4)$ \\
\hline $\mathrm{O} 2-\mathrm{C} 25$ & $1.429(4)$ \\
\hline N1-H1 & 0.8600 \\
\hline N1-C6 & $1.378(4)$ \\
\hline N1-C9 & $1.378(4)$ \\
\hline N2-C1 & $1.467(4)$ \\
\hline N2-C20 & $1.290(4)$ \\
\hline N3-H3 & 0.8600 \\
\hline N3-C21 & $1.372(4)$ \\
\hline N3-C24 & $1.354(4)$ \\
\hline $\mathrm{C} 1-\mathrm{C} 5$ & $1.538(4)$ \\
\hline C1-C18 & $1.539(4)$ \\
\hline C2-H2 & 0.9800 \\
\hline C2-C3 & $1.517(4)$ \\
\hline C2-C17 & $1.518(4)$ \\
\hline C3-H3A & 0.9700 \\
\hline C3-H3B & 0.9700 \\
\hline C3-C4 & $1.528(4)$ \\
\hline C4-H4 & 0.9800 \\
\hline C4-C5 & $1.550(4)$ \\
\hline C4-C16 & $1.534(4)$ \\
\hline C5-H5 & 0.9800 \\
\hline C5-C6 & $1.504(4)$ \\
\hline C6-C7 & $1.368(4)$ \\
\hline C7-H7 & 0.9300 \\
\hline $\mathrm{C} 7-\mathrm{C} 8$ & $1.420(5)$ \\
\hline $\mathrm{C} 8-\mathrm{H} 8$ & 0.9300 \\
\hline C8-C9 & $1.365(5)$ \\
\hline C9-C10 & $1.495(5)$ \\
\hline C10-H10A & 0.9700 \\
\hline C10-H10B & 0.9700 \\
\hline C10-C11 & $1.532(5)$ \\
\hline C11-H11A & 0.9700 \\
\hline C11-H11B & 0.9700 \\
\hline C11-C12 & $1.521(5)$ \\
\hline C12-H12A & 0.9700 \\
\hline C12-H12B & 0.9700 \\
\hline C12-C13 & $1.530(5)$ \\
\hline C13-H13A & 0.9700 \\
\hline C13-H13B & 0.9700 \\
\hline
\end{tabular}




\begin{tabular}{|c|c|}
\hline C13-C14 & $1.523(4)$ \\
\hline C14-H14A & 0.9700 \\
\hline C14-H14B & 0.9700 \\
\hline C14-C15 & $1.522(5)$ \\
\hline C15-H15A & 0.9700 \\
\hline C15-H15B & 0.9700 \\
\hline $\mathrm{C} 15-\mathrm{C} 16$ & $1.529(4)$ \\
\hline C16-H16A & 0.9700 \\
\hline C16-H16B & 0.9700 \\
\hline C17-H17A & 0.9600 \\
\hline C17-H17B & 0.9600 \\
\hline $\mathrm{C} 17-\mathrm{H} 17 \mathrm{C}$ & 0.9600 \\
\hline C18-H18 & 0.9800 \\
\hline C18-C19 & $1.528(4)$ \\
\hline C19-H19A & 0.9700 \\
\hline C19-H19B & 0.9700 \\
\hline C19-C20 & $1.506(4)$ \\
\hline C20-C21 & $1.445(4)$ \\
\hline C21-C22 & $1.375(4)$ \\
\hline C22-H22 & 0.9300 \\
\hline $\mathrm{C} 22-\mathrm{C} 23$ & $1.411(5)$ \\
\hline C23-H23 & 0.9300 \\
\hline C23-C24 & $1.370(5)$ \\
\hline C24-H24 & 0.9300 \\
\hline $\mathrm{C} 25-\mathrm{H} 25 \mathrm{~A}$ & 0.9600 \\
\hline $\mathrm{C} 25-\mathrm{H} 25 \mathrm{~B}$ & 0.9600 \\
\hline $\mathrm{C} 25-\mathrm{H} 25 \mathrm{C}$ & 0.9600 \\
\hline O3-H3C & 0.8397 \\
\hline O3-H3D & 0.8400 \\
\hline $\mathrm{C} 1-\mathrm{O} 1-\mathrm{C} 2$ & $117.9(2)$ \\
\hline $\mathrm{C} 18-\mathrm{O} 2-\mathrm{C} 25$ & $112.6(2)$ \\
\hline C6-N1-H1 & 124.9 \\
\hline C9-N1-H1 & 124.9 \\
\hline C9-N1-C6 & $110.3(3)$ \\
\hline C20-N2-C1 & $108.2(2)$ \\
\hline C21-N3-H3 & 125.4 \\
\hline C24-N3-H3 & 125.4 \\
\hline C24-N3-C21 & 109.3(3) \\
\hline $\mathrm{O} 1-\mathrm{C} 1-\mathrm{N} 2$ & $109.9(2)$ \\
\hline $\mathrm{O} 1-\mathrm{C} 1-\mathrm{C} 5$ & $111.8(2)$ \\
\hline $\mathrm{O} 1-\mathrm{C} 1-\mathrm{C} 18$ & $105.0(2)$ \\
\hline N2-C1-C5 & $111.1(2)$ \\
\hline $\mathrm{N} 2-\mathrm{C} 1-\mathrm{C} 18$ & $104.8(2)$ \\
\hline
\end{tabular}




\begin{tabular}{|c|c|}
\hline C5-C1-C18 & $113.9(2)$ \\
\hline $\mathrm{O} 1-\mathrm{C} 2-\mathrm{H} 2$ & 106.5 \\
\hline $\mathrm{O} 1-\mathrm{C} 2-\mathrm{C} 3$ & $109.6(2)$ \\
\hline $\mathrm{O} 1-\mathrm{C} 2-\mathrm{C} 17$ & $113.0(3)$ \\
\hline C3-C2-H2 & 106.5 \\
\hline $\mathrm{C} 3-\mathrm{C} 2-\mathrm{C} 17$ & $114.2(3)$ \\
\hline C17-C2-H2 & 106.5 \\
\hline C2-C3-H3A & 109.1 \\
\hline C2-C3-H3B & 109.1 \\
\hline C2-C3-C4 & $112.6(2)$ \\
\hline H3A-C3-H3B & 107.8 \\
\hline C4-C3-H3A & 109.1 \\
\hline C4-C3-H3B & 109.1 \\
\hline C3-C4-H4 & 108.1 \\
\hline C3-C4-C5 & $109.6(2)$ \\
\hline $\mathrm{C} 3-\mathrm{C} 4-\mathrm{C} 16$ & $111.0(2)$ \\
\hline C5-C4-H4 & 108.1 \\
\hline C16-C4-H4 & 108.1 \\
\hline $\mathrm{C} 16-\mathrm{C} 4-\mathrm{C} 5$ & $111.9(2)$ \\
\hline $\mathrm{C} 1-\mathrm{C} 5-\mathrm{C} 4$ & $111.9(2)$ \\
\hline C1-C5-H5 & 107.0 \\
\hline C4-C5-H5 & 107.0 \\
\hline C6-C5-C1 & $113.2(2)$ \\
\hline C6-C5-C4 & $110.4(2)$ \\
\hline C6-C5-H5 & 107.0 \\
\hline N1-C6-C5 & $122.7(2)$ \\
\hline C7-C6-N1 & $107.0(3)$ \\
\hline C7-C6-C5 & $129.6(3)$ \\
\hline C6-C7-H7 & 126.1 \\
\hline C6-C7-C8 & $107.8(3)$ \\
\hline C8-C7-H7 & 126.1 \\
\hline C7-C8-H8 & 126.0 \\
\hline C9-C8-C7 & $108.1(3)$ \\
\hline C9-C8-H8 & 126.0 \\
\hline N1-C9-C10 & $122.0(3)$ \\
\hline C8-C9-N1 & $106.9(3)$ \\
\hline C8-C9-C10 & $130.7(3)$ \\
\hline C9-C10-H10A & 108.4 \\
\hline C9-C10-H10B & 108.4 \\
\hline C9-C10-C11 & $115.6(3)$ \\
\hline H10A-C10-H10B & 107.4 \\
\hline C11-C10-H10A & 108.4 \\
\hline C11-C10-H10B & 108.4 \\
\hline C10-C11-H11A & 108.6 \\
\hline
\end{tabular}




\begin{tabular}{ll} 
C10-C11-H11B & 108.6 \\
H11A-C11-H11B & 107.6 \\
C12-C11-C10 & $114.7(3)$ \\
C12-C11-H11A & 108.6 \\
C12-C11-H11B & 108.6 \\
C11-C12-H12A & 108.6 \\
C11-C12-H12B & 108.6 \\
C11-C12-C13 & $114.8(3)$ \\
H12A-C12-H12B & 107.5 \\
C13-C12-H12A & 108.6 \\
C13-C12-H12B & 108.6 \\
C12-C13-H13A & 109.0 \\
C12-C13-H13B & 109.0 \\
H13A-C13-H13B & 107.8 \\
C14-C13-C12 & $112.8(3)$ \\
C14-C13-H13A & 109.0 \\
C14-C13-H13B & 109.0 \\
C13-C14-H14A & 108.7 \\
C13-C14-H14B & 108.7 \\
H14A-C14-H14B & 107.6 \\
C15-C14-C13 & $114.2(3)$ \\
C15-C14-H14A & 108.7 \\
C15-C14-H14B & 108.7 \\
C14-C15-H15A & 108.7 \\
C14-C15-H15B & 108.7 \\
C14-C15-C16 & $114.3(3)$ \\
H15A-C15-H15B & 107.6 \\
C16-C15-H15A & 108.7 \\
C16-C15-H15B & 108.7 \\
C4-C16-H16A & 108.6 \\
C4-C16-H16B & 108.6 \\
C15-C16-C4 & $114.5(3)$ \\
C15-C16-H16A & 108.6 \\
C15-C16-H16B & 108.6 \\
H16A-C16-H16B & 107.6 \\
C2-C17-H17A & 109.5 \\
C2-C17-H17B & 109.5 \\
C2-C17-H17C & 109.5 \\
H17A-C17-H17B & 109.5 \\
H17A-C17-H17C & 109.5 \\
O2-C18-H18 & 109.5 \\
O2-C18-C19 & $111.2(2)$ \\
& 108.9 \\
H17-H17C & $115.7(2)$ \\
\hline
\end{tabular}




\begin{tabular}{ll} 
C1-C18-H18 & 108.9 \\
C19-C18-C1 & $103.0(2)$ \\
C19-C18-H18 & 108.9 \\
C18-C19-H19A & 111.8 \\
C18-C19-H19B & 111.8 \\
H19A-C19-H19B & 109.5 \\
C20-C19-C18 & $100.0(2)$ \\
C20-C19-H19A & 111.8 \\
C20-C19-H19B & 111.8 \\
N2-C20-C19 & $115.6(3)$ \\
N2-C20-C21 & $121.5(3)$ \\
C21-C20-C19 & $122.9(3)$ \\
N3-C21-C20 & $121.2(3)$ \\
N3-C21-C22 & $107.3(3)$ \\
C22-C21-C20 & $131.5(3)$ \\
C21-C22-H22 & 126.0 \\
C21-C22-C23 & $107.9(3)$ \\
C23-C22-H22 & 126.0 \\
C22-C23-H23 & 126.8 \\
C24-C23-C22 & $106.5(3)$ \\
C24-C23-H23 & 126.8 \\
N3-C24-C23 & $109.0(3)$ \\
N3-C24-H24 & 125.5 \\
C23-C24-H24 & 125.5 \\
O2-C25-H25A & 109.5 \\
O2-C25-H25B & 109.5 \\
O2-C25-H25C & 109.5 \\
H25A-C25-H25B & 109.5 \\
H25A-C25-H25C & 109.5 \\
H25B-C25-H25C & 109.5 \\
H3C-O3-H3D & 97.3 \\
& \\
\hline
\end{tabular}

Symmetry transformations used to generate equivalent atoms: 
Table 4. Anisotropic displacement parameters $\left(\AA^{2} \times 10^{3}\right)$ for 1. The anisotropic displacement factor exponent takes the form: $-2 \mathrm{p}^{2}\left[\mathrm{~h}^{2} \mathrm{a}^{* 2} \mathrm{U}^{11}+\ldots+2 \mathrm{hk} \mathrm{a}^{*} \mathrm{~b}^{*} \mathrm{U}^{12}\right]$

\begin{tabular}{|c|c|c|c|c|c|c|}
\hline & $\mathrm{U}^{11}$ & $\mathrm{U}^{22}$ & $\mathrm{U}^{33}$ & $\mathrm{U}^{23}$ & $\mathrm{U}^{13}$ & $\mathrm{U}^{12}$ \\
\hline $\mathrm{O} 1$ & $23(1)$ & $26(1)$ & $22(1)$ & $2(1)$ & $-3(1)$ & $-6(1)$ \\
\hline $\mathrm{O} 2$ & $24(1)$ & $30(1)$ & $34(1)$ & $7(1)$ & $7(1)$ & $-1(1)$ \\
\hline N1 & $26(1)$ & $26(1)$ & $21(1)$ & $-1(1)$ & $-2(1)$ & $0(1)$ \\
\hline $\mathrm{N} 2$ & $22(1)$ & $23(1)$ & $22(1)$ & $-3(1)$ & $-1(1)$ & $-1(1)$ \\
\hline N3 & $29(1)$ & $24(1)$ & $33(1)$ & $2(1)$ & $2(1)$ & $1(1)$ \\
\hline $\mathrm{C} 1$ & $19(1)$ & $22(1)$ & $19(1)$ & $0(1)$ & $0(1)$ & $1(1)$ \\
\hline $\mathrm{C} 2$ & $28(2)$ & $30(2)$ & $22(1)$ & $4(1)$ & $-3(1)$ & $-6(1)$ \\
\hline $\mathrm{C} 3$ & $28(2)$ & $28(2)$ & $24(1)$ & $5(1)$ & $-2(1)$ & $-2(1)$ \\
\hline $\mathrm{C} 4$ & $24(2)$ & $20(1)$ & $25(1)$ & $1(1)$ & $0(1)$ & $-1(1)$ \\
\hline $\mathrm{C} 5$ & $20(1)$ & $22(1)$ & $22(1)$ & $0(1)$ & $2(1)$ & $1(1)$ \\
\hline C6 & $26(2)$ & $19(1)$ & $24(1)$ & $-1(1)$ & $-3(1)$ & $1(1)$ \\
\hline C7 & $35(2)$ & $29(2)$ & $24(2)$ & $1(1)$ & $1(1)$ & $4(2)$ \\
\hline $\mathrm{C} 8$ & $43(2)$ & $34(2)$ & $21(1)$ & $0(1)$ & $-8(2)$ & $3(2)$ \\
\hline C9 & $32(2)$ & $25(2)$ & $29(2)$ & $-1(1)$ & $-11(1)$ & $0(1)$ \\
\hline $\mathrm{C} 10$ & $32(2)$ & $36(2)$ & $38(2)$ & $-2(2)$ & $-16(2)$ & $1(2)$ \\
\hline C11 & $27(2)$ & $44(2)$ & $48(2)$ & $-12(2)$ & $-11(2)$ & $0(2)$ \\
\hline C12 & $30(2)$ & $42(2)$ & $49(2)$ & $1(2)$ & $2(2)$ & $-4(2)$ \\
\hline C13 & $33(2)$ & $27(2)$ & $40(2)$ & $-3(1)$ & $-3(2)$ & $-2(2)$ \\
\hline C14 & $38(2)$ & $27(2)$ & $35(2)$ & $-2(1)$ & $2(2)$ & $-10(2)$ \\
\hline C15 & $43(2)$ & $21(2)$ & $35(2)$ & $3(1)$ & $-4(2)$ & $-4(2)$ \\
\hline C16 & $28(2)$ & $22(1)$ & $30(2)$ & $-2(1)$ & $-3(1)$ & $4(1)$ \\
\hline C17 & $39(2)$ & $36(2)$ & $25(2)$ & $-1(1)$ & $4(2)$ & $-10(2)$ \\
\hline C18 & $23(1)$ & $24(1)$ & $21(1)$ & $1(1)$ & $1(1)$ & $-1(1)$ \\
\hline C19 & $27(2)$ & $20(1)$ & $26(1)$ & $-1(1)$ & $2(1)$ & $-1(1)$ \\
\hline $\mathrm{C} 20$ & $23(2)$ & $23(1)$ & $21(1)$ & $-2(1)$ & $-2(1)$ & $-2(1)$ \\
\hline $\mathrm{C} 21$ & $23(2)$ & $25(1)$ & $25(2)$ & $-2(1)$ & $-1(1)$ & $1(1)$ \\
\hline $\mathrm{C} 22$ & $31(2)$ & $26(2)$ & $30(2)$ & $-2(1)$ & $1(2)$ & $-3(1)$ \\
\hline $\mathrm{C} 23$ & $38(2)$ & $25(2)$ & $35(2)$ & $-2(1)$ & $1(2)$ & $10(2)$ \\
\hline $\mathrm{C} 24$ & $29(2)$ & $35(2)$ & $39(2)$ & $-1(2)$ & $4(2)$ & $9(2)$ \\
\hline $\mathrm{C} 25$ & $37(2)$ & $38(2)$ & $43(2)$ & $10(2)$ & $18(2)$ & $-2(2)$ \\
\hline $\mathrm{O} 3$ & $24(1)$ & $41(1)$ & $48(1)$ & $4(1)$ & $-1(1)$ & $-2(1)$ \\
\hline
\end{tabular}


Table 5. Hydrogen coordinates $\left(\mathrm{x} 10^{4}\right)$ and isotropic displacement parameters $\left(\AA^{2} \times 10^{3}\right)$ for 1 .

\begin{tabular}{|c|c|c|c|c|}
\hline & $\mathrm{x}$ & $\mathrm{y}$ & $\mathrm{z}$ & $\mathrm{U}(\mathrm{eq})$ \\
\hline H1 & 1057 & 5226 & 6042 & 29 \\
\hline H3 & 177 & 7311 & 6865 & 34 \\
\hline $\mathrm{H} 2$ & 6259 & 5393 & 7674 & 32 \\
\hline $\mathrm{H} 3 \mathrm{~A}$ & 4814 & 3833 & 7468 & 32 \\
\hline H3B & 6221 & 4069 & 7000 & 32 \\
\hline $\mathrm{H} 4$ & 2851 & 4420 & 6775 & 28 \\
\hline H5 & 5448 & 4815 & 5941 & 26 \\
\hline H7 & 4387 & 4687 & 4796 & 36 \\
\hline H8 & 1605 & 4507 & 4315 & 39 \\
\hline $\mathrm{H} 10 \mathrm{~A}$ & -1547 & 4990 & 4765 & 43 \\
\hline H10B & -1610 & 5161 & 5474 & 43 \\
\hline H11A & -2955 & 3608 & 5165 & 47 \\
\hline H11B & -1282 & 3167 & 4930 & 47 \\
\hline $\mathrm{H} 12 \mathrm{~A}$ & -2267 & 2599 & 5943 & 48 \\
\hline H12B & -1615 & 3679 & 6173 & 48 \\
\hline H13A & 228 & 1947 & 5814 & 40 \\
\hline H13B & 1013 & 3044 & 5690 & 40 \\
\hline H14A & 691 & 3417 & 6763 & 40 \\
\hline H14B & 170 & 2250 & 6855 & 40 \\
\hline H15A & 2775 & 1698 & 6544 & 39 \\
\hline H15B & 2883 & 2411 & 7126 & 39 \\
\hline H16A & 3535 & 3155 & 5930 & 32 \\
\hline H16B & 4920 & 2807 & 6382 & 32 \\
\hline H17A & 3980 & 6474 & 7882 & 50 \\
\hline H17B & 3807 & 5341 & 8148 & 50 \\
\hline $\mathrm{H} 17 \mathrm{C}$ & 2870 & 5653 & 7548 & 50 \\
\hline H18 & 5095 & 6750 & 5515 & 27 \\
\hline H19A & 5458 & 8311 & 6423 & 30 \\
\hline H19B & 4581 & 8419 & 5779 & 30 \\
\hline $\mathrm{H} 22$ & 2457 & 9815 & 6289 & 35 \\
\hline $\mathrm{H} 23$ & -348 & 10331 & 6653 & 39 \\
\hline H24 & -1713 & 8719 & 7004 & 41 \\
\hline $\mathrm{H} 25 \mathrm{~A}$ & 7536 & 7202 & 5088 & 59 \\
\hline $\mathrm{H} 25 \mathrm{~B}$ & 9121 & 7283 & 5490 & 59 \\
\hline $\mathrm{H} 25 \mathrm{C}$ & 7761 & 8143 & 5540 & 59 \\
\hline $\mathrm{H} 3 \mathrm{C}$ & 9198 & 5374 & 7153 & 57 \\
\hline H3D & 8250 & 5849 & 6792 & 57 \\
\hline
\end{tabular}


Table 6. Torsion angles $\left[{ }^{\circ}\right]$ for $\mathbf{1}$.

\begin{tabular}{|c|c|}
\hline $\mathrm{O} 1-\mathrm{C} 1-\mathrm{C} 5-\mathrm{C} 4$ & $48.8(3)$ \\
\hline O1-C1-C5-C6 & $174.3(2)$ \\
\hline $\mathrm{O} 1-\mathrm{C} 1-\mathrm{C} 18-\mathrm{O} 2$ & $37.1(3)$ \\
\hline $\mathrm{O} 1-\mathrm{C} 1-\mathrm{C} 18-\mathrm{C} 19$ & $-87.5(3)$ \\
\hline O1-C2-C3-C4 & $-54.6(3)$ \\
\hline O2-C18-C19-C20 & $-147.4(2)$ \\
\hline N1-C6-C7-C8 & $-0.7(3)$ \\
\hline N1-C9-C10-C11 & $-92.4(4)$ \\
\hline N2-C1-C5-C4 & $-74.4(3)$ \\
\hline N2-C1-C5-C6 & $51.2(3)$ \\
\hline N2-C1-C18-O2 & $153.0(2)$ \\
\hline N2-C1-C18-C19 & $28.4(3)$ \\
\hline N2-C20-C21-N3 & $-1.5(4)$ \\
\hline N2-C20-C21-C22 & $177.7(3)$ \\
\hline N3-C21-C22-C23 & $0.3(3)$ \\
\hline $\mathrm{C} 1-\mathrm{O} 1-\mathrm{C} 2-\mathrm{C} 3$ & $54.2(3)$ \\
\hline $\mathrm{C} 1-\mathrm{O} 1-\mathrm{C} 2-\mathrm{C} 17$ & $-74.4(3)$ \\
\hline C1-N2-C20-C19 & $1.8(3)$ \\
\hline C1-N2-C20-C21 & $-178.4(2)$ \\
\hline C1-C5-C6-N1 & $-76.0(3)$ \\
\hline C1-C5-C6-C7 & $115.4(3)$ \\
\hline C1-C18-C19-C20 & $-25.8(3)$ \\
\hline $\mathrm{C} 2-\mathrm{O} 1-\mathrm{C} 1-\mathrm{N} 2$ & $71.9(3)$ \\
\hline $\mathrm{C} 2-\mathrm{O} 1-\mathrm{C} 1-\mathrm{C} 5$ & $-51.9(3)$ \\
\hline $\mathrm{C} 2-\mathrm{O} 1-\mathrm{C} 1-\mathrm{C} 18$ & $-175.9(2)$ \\
\hline C2-C3-C4-C5 & $54.6(3)$ \\
\hline $\mathrm{C} 2-\mathrm{C} 3-\mathrm{C} 4-\mathrm{C} 16$ & $178.7(3)$ \\
\hline C3-C4-C5-C1 & $-50.9(3)$ \\
\hline C3-C4-C5-C6 & $-177.9(2)$ \\
\hline C3-C4-C16-C15 & $86.8(3)$ \\
\hline C4-C5-C6-N1 & $50.4(4)$ \\
\hline C4-C5-C6-C7 & $-118.2(3)$ \\
\hline $\mathrm{C} 5-\mathrm{C} 1-\mathrm{C} 18-\mathrm{O} 2$ & $-85.4(3)$ \\
\hline C5-C1-C18-C19 & $149.9(2)$ \\
\hline C5-C4-C16-C15 & $-150.5(3)$ \\
\hline C5-C6-C7-C8 & $169.2(3)$ \\
\hline C6-N1-C9-C8 & $-1.4(3)$ \\
\hline C6-N1-C9-C10 & $172.5(3)$ \\
\hline C6-C7-C8-C9 & $-0.1(4)$ \\
\hline C7-C8-C9-N1 & $0.9(4)$ \\
\hline C7-C8-C9-C10 & $-172.3(3)$ \\
\hline C8-C9-C10-C11 & $79.9(5)$ \\
\hline
\end{tabular}




$\begin{array}{lc}\text { C9-N1-C6-C5 } & -169.5(3) \\ \text { C9-N1-C6-C7 } & 1.3(3) \\ \text { C9-C10-C11-C12 } & 66.3(4) \\ \text { C10-C11-C12-C13 } & -82.7(4) \\ \text { C11-C12-C13-C14 } & 151.1(3) \\ \text { C12-C13-C14-C15 } & -171.1(3) \\ \text { C13-C14-C15-C16 } & 62.1(4) \\ \text { C14-C15-C16-C4 } & 67.4(4) \\ \text { C16-C4-C5-C1 } & -174.5(2) \\ \text { C16-C4-C5-C6 } & 58.5(3) \\ \text { C17-C2-C3-C4 } & 73.4(3) \\ \text { C18-C1-C5-C4 } & 167.6(2) \\ \text { C18-C1-C5-C6 } & -66.8(3) \\ \text { C18-C19-C20-N2 } & 16.3(3) \\ \text { C18-C19-C20-C21 } & -163.5(3) \\ \text { C19-C20-C21-N3 } & 178.3(3) \\ \text { C19-C20-C21-C22 } & -2.4(5) \\ \text { C20-N2-C1-O1 } & 93.2(3) \\ \text { C20-N2-C1-C5 } & -142.6(2) \\ \text { C20-N2-C1-C18 } & -19.2(3) \\ \text { C20-C21-C22-C23 } & -179.0(3) \\ \text { C21-N3-C24-C23 } & 0.0(4) \\ \text { C21-C22-C23-C24 } & -0.3(4) \\ \text { C22-C23-C24-N3 } & 0.2(4) \\ \text { C24-N3-C21-C20 } & 179.2(3) \\ \text { C24-N3-C21-C22 } & -0.2(3) \\ \text { C25-O2-C18-C1 } & 171.1(2) \\ \text { C25-O2-C18-C19 } & -71.8(3) \\ & \\ & \end{array}$

Symmetry transformations used to generate equivalent atoms:

Table 7. Hydrogen bonds for $1 \quad$ [ $\AA$ and $\left.{ }^{\circ}\right]$.

\begin{tabular}{lcccc}
\hline D-H...A & $\mathrm{d}(\mathrm{D}-\mathrm{H})$ & $\mathrm{d}(\mathrm{H} \ldots \mathrm{A})$ & $\mathrm{d}(\mathrm{D} \ldots \mathrm{A})$ & $<$ (DHA) \\
\hline N1-H1...O3\#1 & 0.86 & 2.40 & $3.238(4)$ & 166.3 \\
N3-H3...O3\#1 & 0.86 & 2.11 & $2.940(3)$ & 163.0 \\
O3-H3D...O1 & 0.84 & 2.12 & $2.940(3)$ & 164.6 \\
O3-H3D...O2 & 0.84 & 2.38 & $2.919(3)$ & 122.2
\end{tabular}

Symmetry transformations used to generate equivalent atoms:

\#1 x-1,y,z 


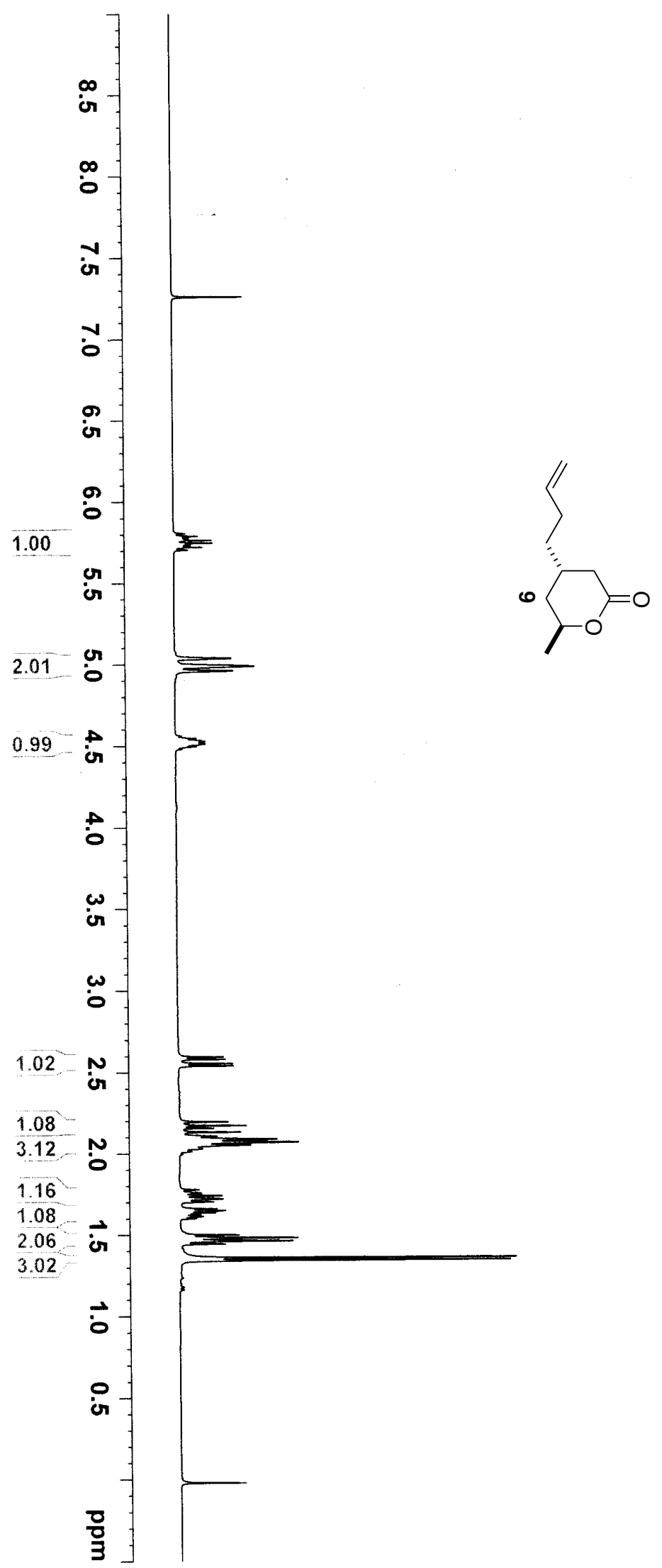

5.791
5.782 5.774 $\begin{array}{r}5.765 \\ -5.748 \\ \hline\end{array}$ 5.739 $-5.731$ 5.723
5.706 5.039 $\begin{array}{r}5.036 \\ -4.988 \\ \hline\end{array}$ $-4.962$ 4.561 4.545 $-4.534$ 4.529
-4.522 I. 4.522 $\left[\begin{array}{r}4.519 \\ 4.512\end{array}\right.$ $-4.507$ 4.496 4.481 2.596
-2.582
-2.556 2.556 r.541 [2.197 r 2.173 2.156
-2.132 2.132 2.107 2.088 2.070 2.052 $-2.036$ 2.029
2.020 2.020
-2.013 1.778 $-1.760$ $-1.756$ $-1.742$ $-1.720$

$-1.703$

1.661

$\left[\begin{array}{r}1.650 \\ 1.636\end{array}\right.$

$\left[\begin{array}{l}1.626 \\ 1.614\end{array}\right.$

$-1.614$

- 1.600 1.500

$-1.481$

$-1.462$

$\left[\begin{array}{r}1.358 \\ 1.342\end{array}\right.$ 

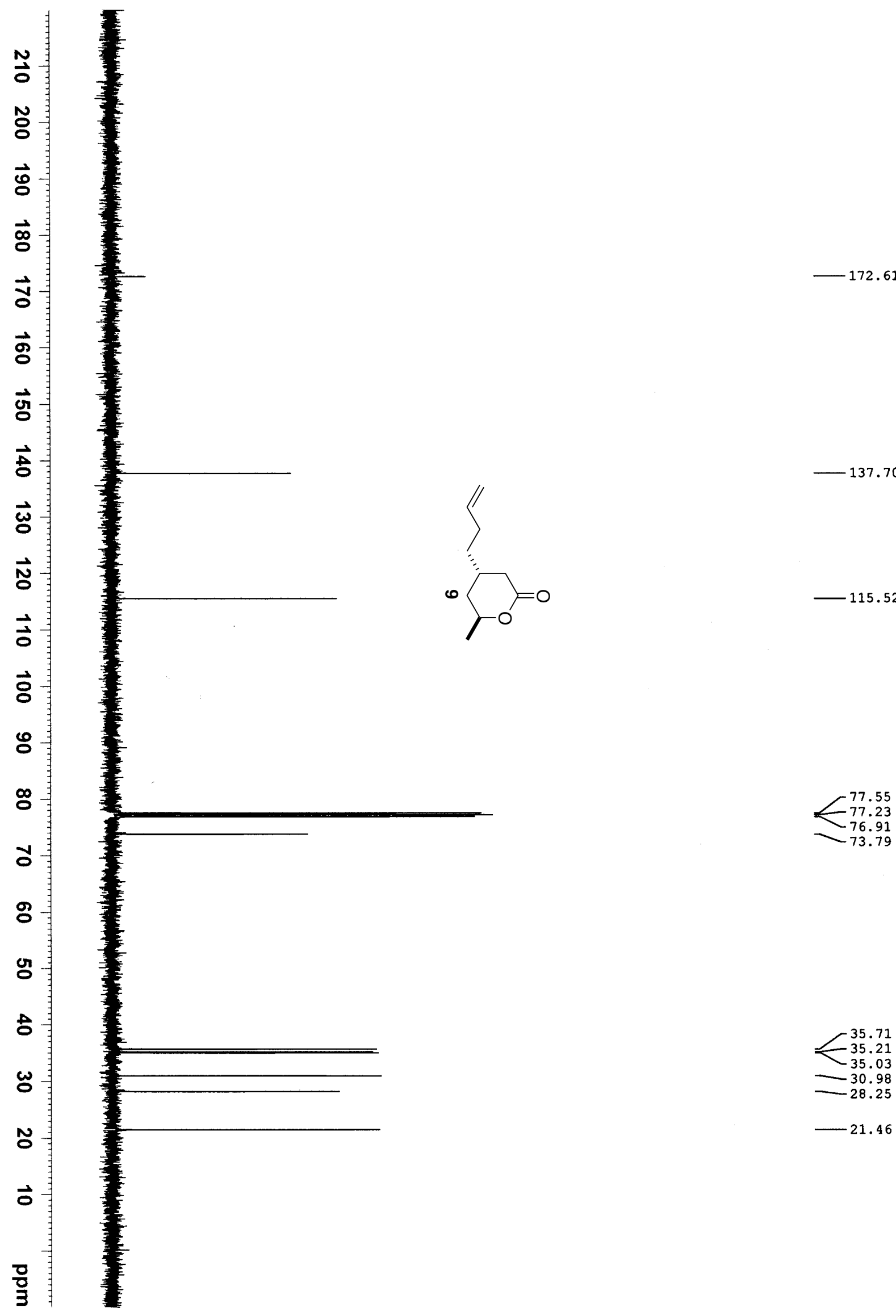

$-35.71$

35.21
-35.03

35.03

28.25

$-21.46$ 

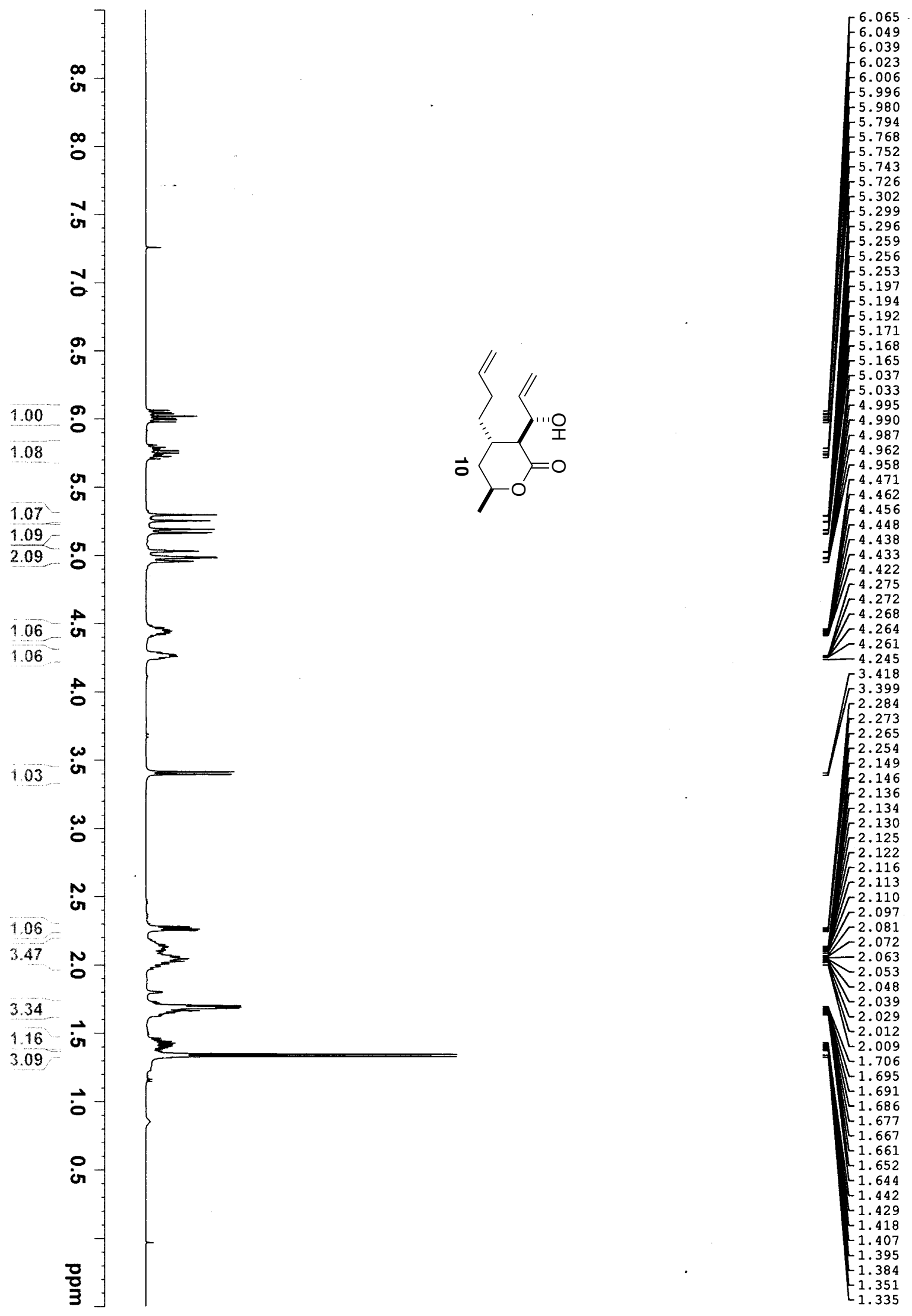

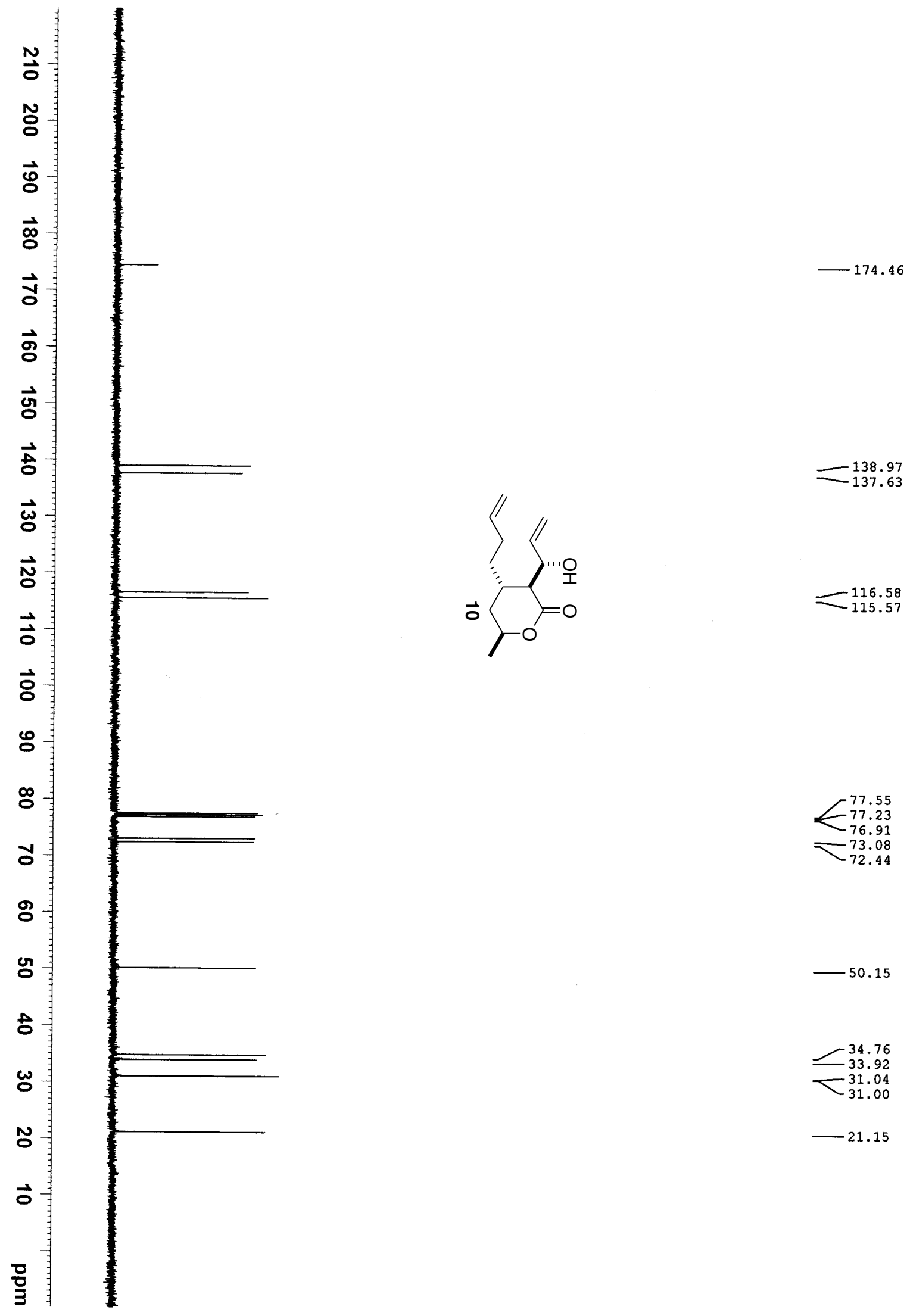

77.23
-76.91

76.91

$-72.44$

$-50.15$

$-34.76$

33.92
-31.04

$-31.00$

$-21.15$ 

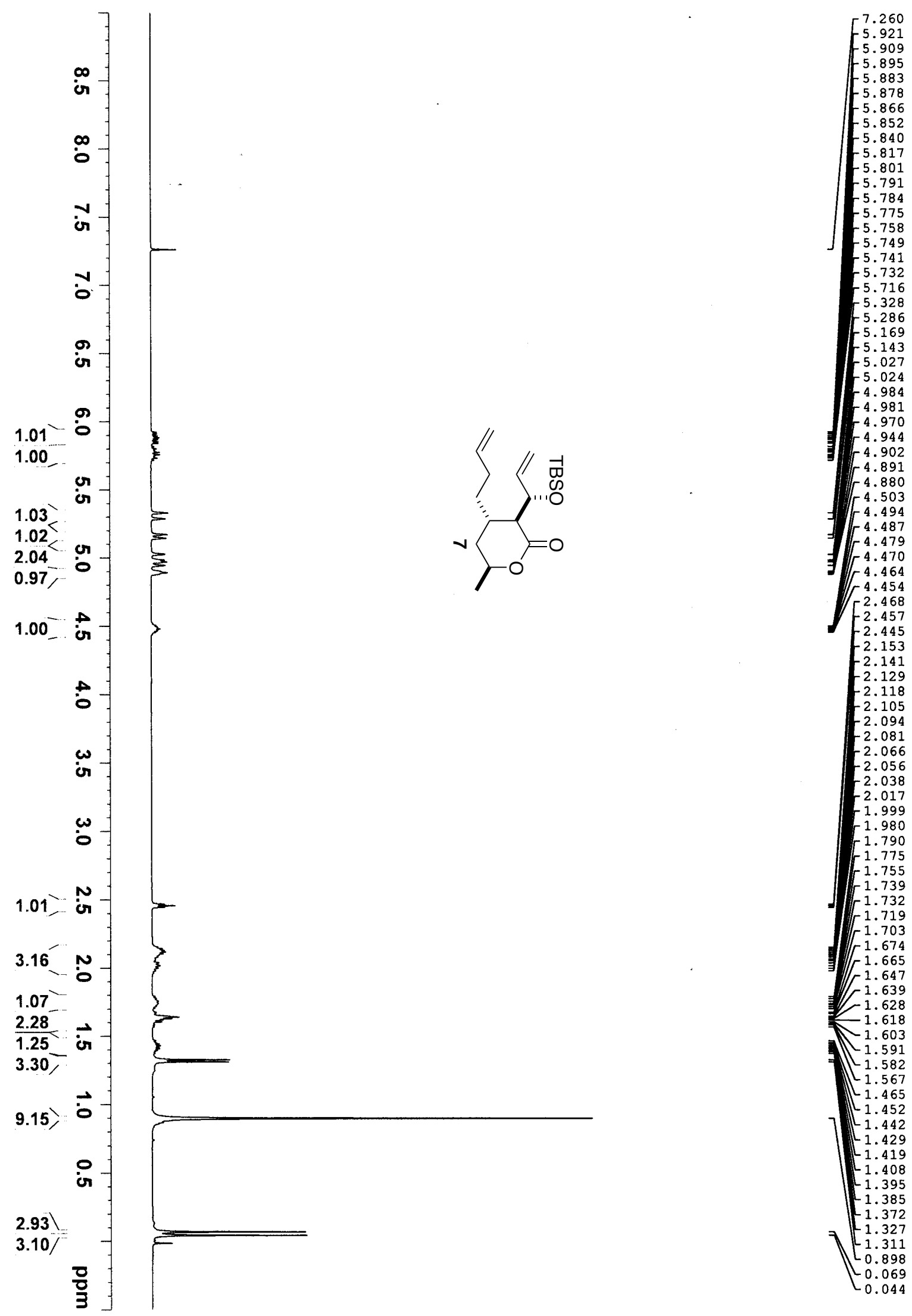


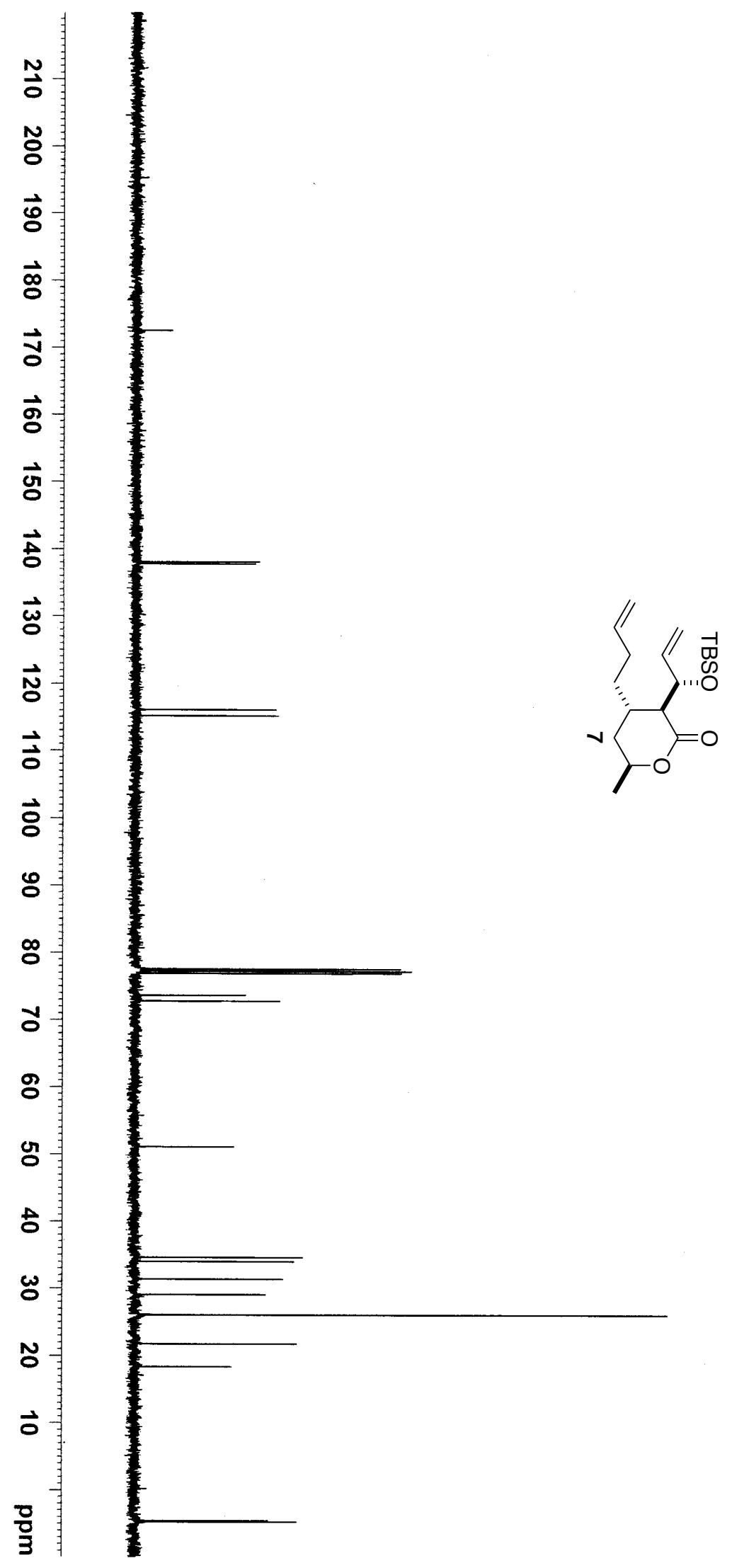

$-172.50$

138.08
+137.78

-116.07
-115.16

77.55
-77.23

77.23
76.91

$-73.64$

72.78

$-51.12$

$-34.60$

$-33.99$

$-31.36$

29.06
-26.05

-21.73
-18.35 

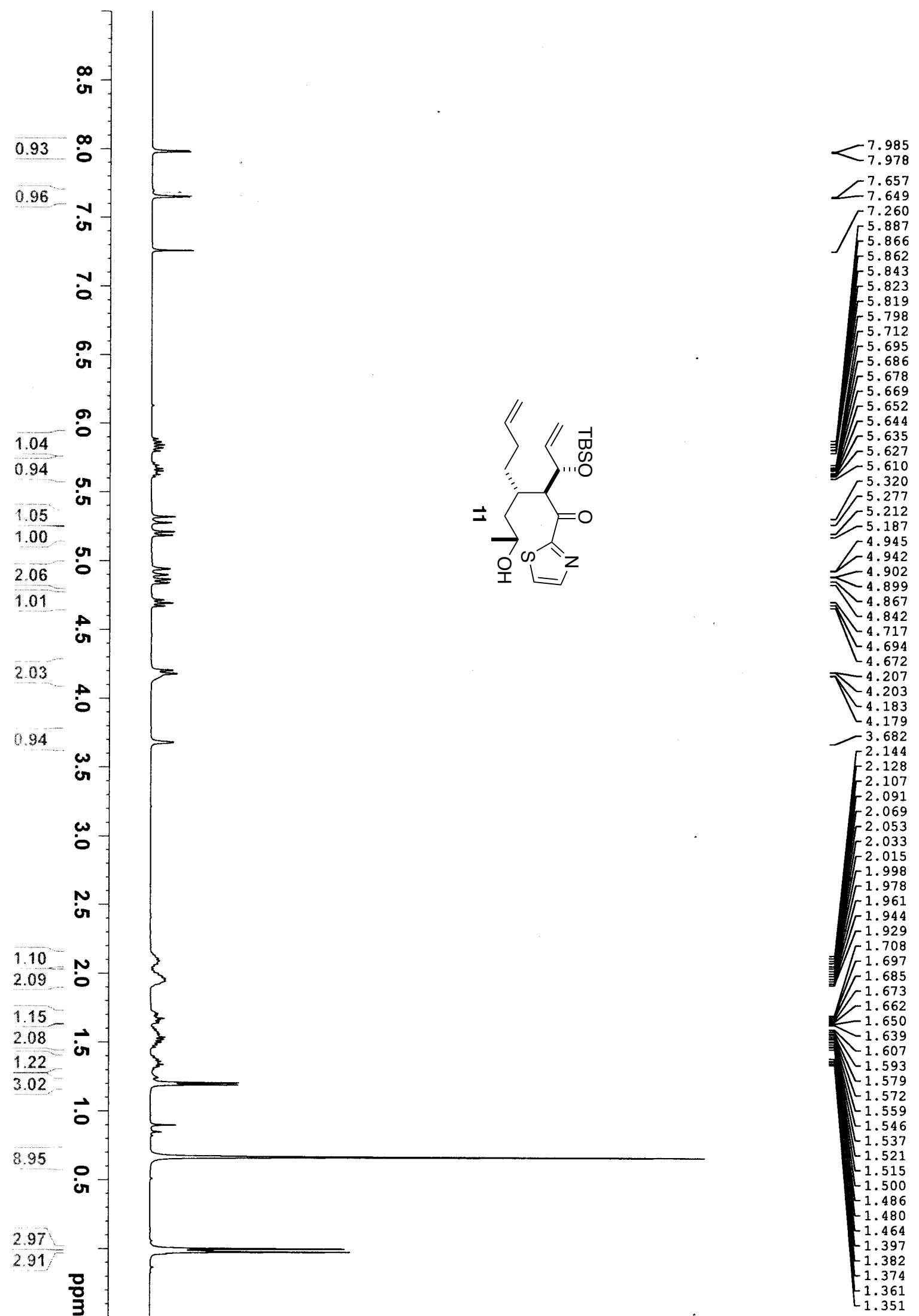


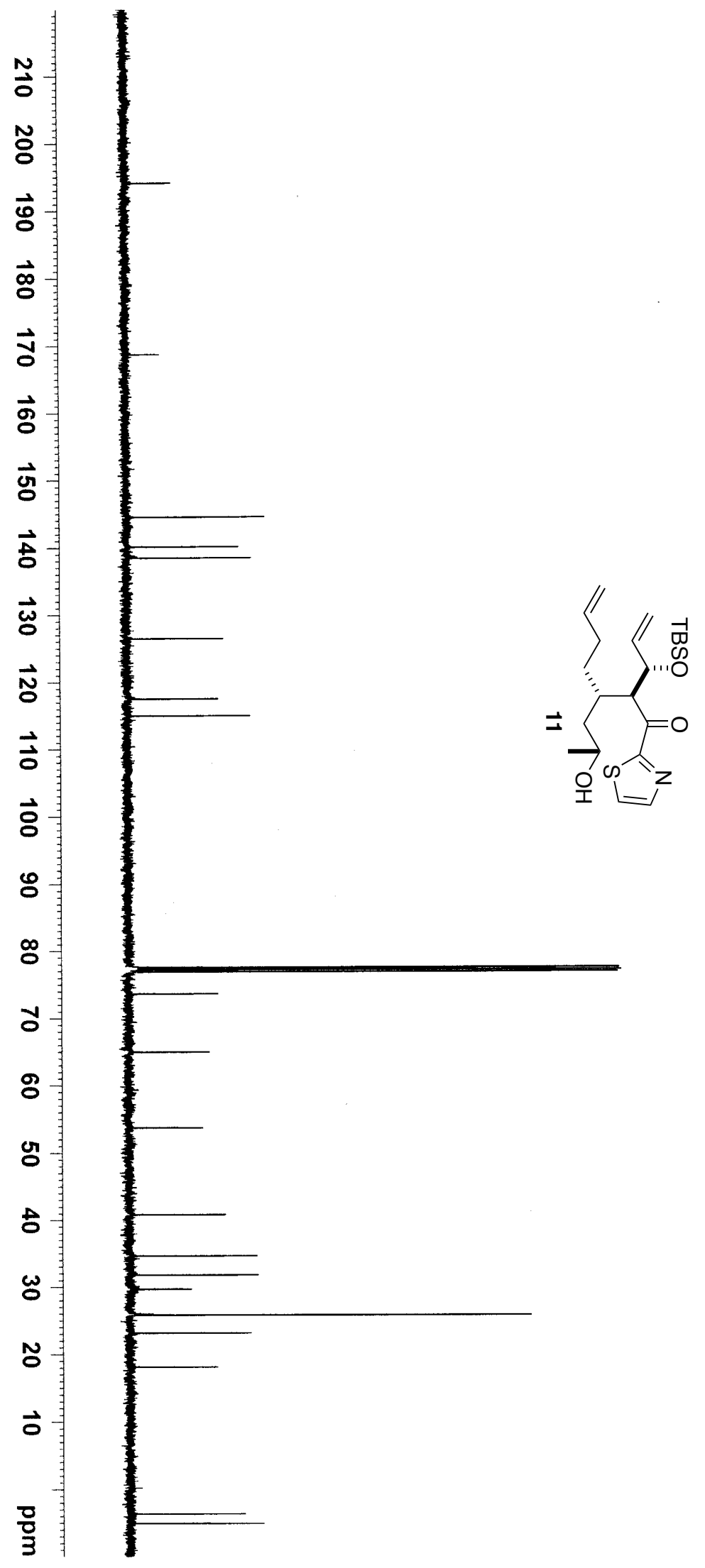

$-194.09$

$-168.70$

144.53

$-140.13$ 138.47

$-126.46$

$-117.51$

115.00

$\begin{array}{r}77.54 \\ -77.23 \\ \hline\end{array}$

$-76.91$

$-73.65$

$-64.97$

$-53.72$

$-40.81$

$-34.65$

31.78
$-\quad 29.70$

$-25.83$

$-23.16$

$-18.11$

-3.66
--5.08 


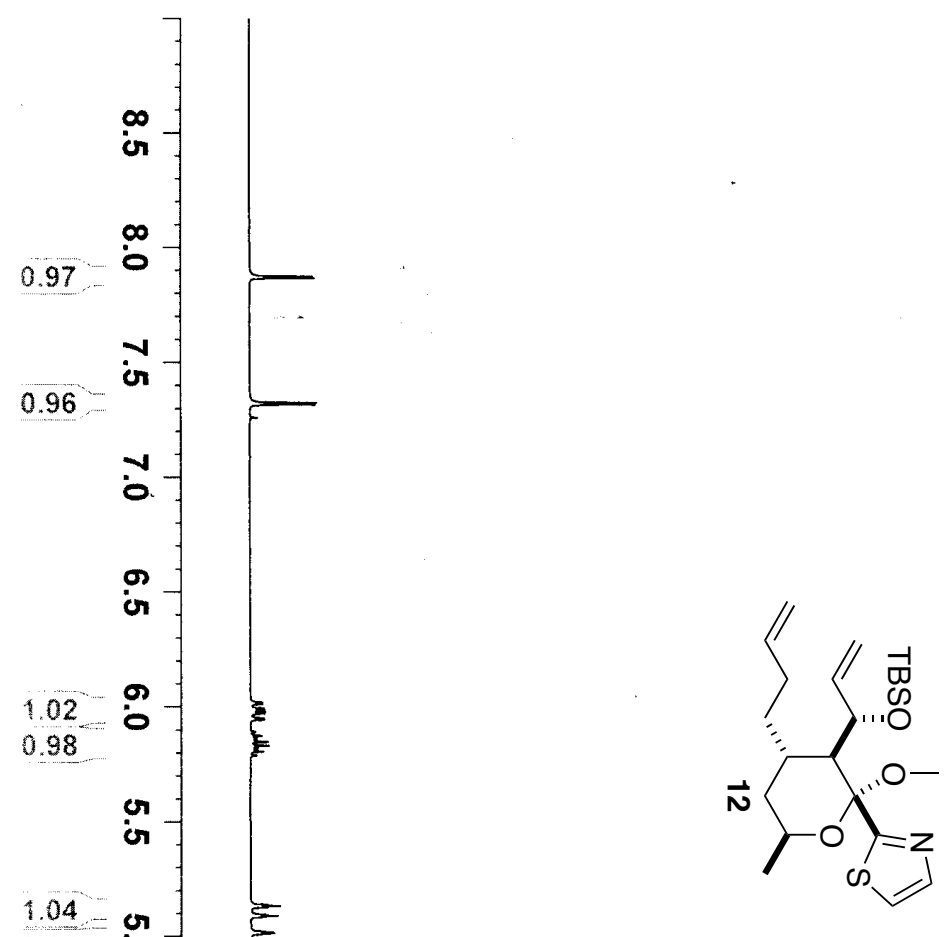

7.874
-7.866

7.326

$-7.318$

6.021

$-6.013$

$-5.987$

$-5.971$

$-5.952$

$-5.875$

$-5.849$

$-5.832$

5.807

5.138

5.133

5.127

5.096

$-5.084$

$-5.018$

$-5.014$

$-4.975$

4.971

4.960

4.960

4.950
-4.939

4.933

4.927

4.921

4.919

4.916
4.896

4.896

$\begin{array}{r}4.893 \\ -4.891 \\ \hline\end{array}$

4.003

4.003
-3.996

3.996
3.988

3.981

3.973

3.966

3.957
3.951
3.678

3. 678

3.672

3.672
-3.664

$-3.656$

3.650

2.950
$r^{2} .333$
$r^{2} .324$

2.324
-2.145

$F^{2.145}$

2.109

2.092

2.074
2.058

2.058

$r^{1.860}$

$F^{1.843}$

1.826

1.819
-1.809

$-1.802$

$-1.794$

$-1.789$

$-1.778$

1.774

1.759
-1.748

1.748

1.744
1.735

$-1.735$

$-1.728$

$-1.714$

$-1.702$

1.690
-1.281

$-1.281$

$-1.252$

$-1.237$

$\left[\begin{array}{l}0.844 \\ -0.021\end{array}\right.$

$\left[\begin{array}{l}-0.021 \\ -0.151\end{array}\right.$ 


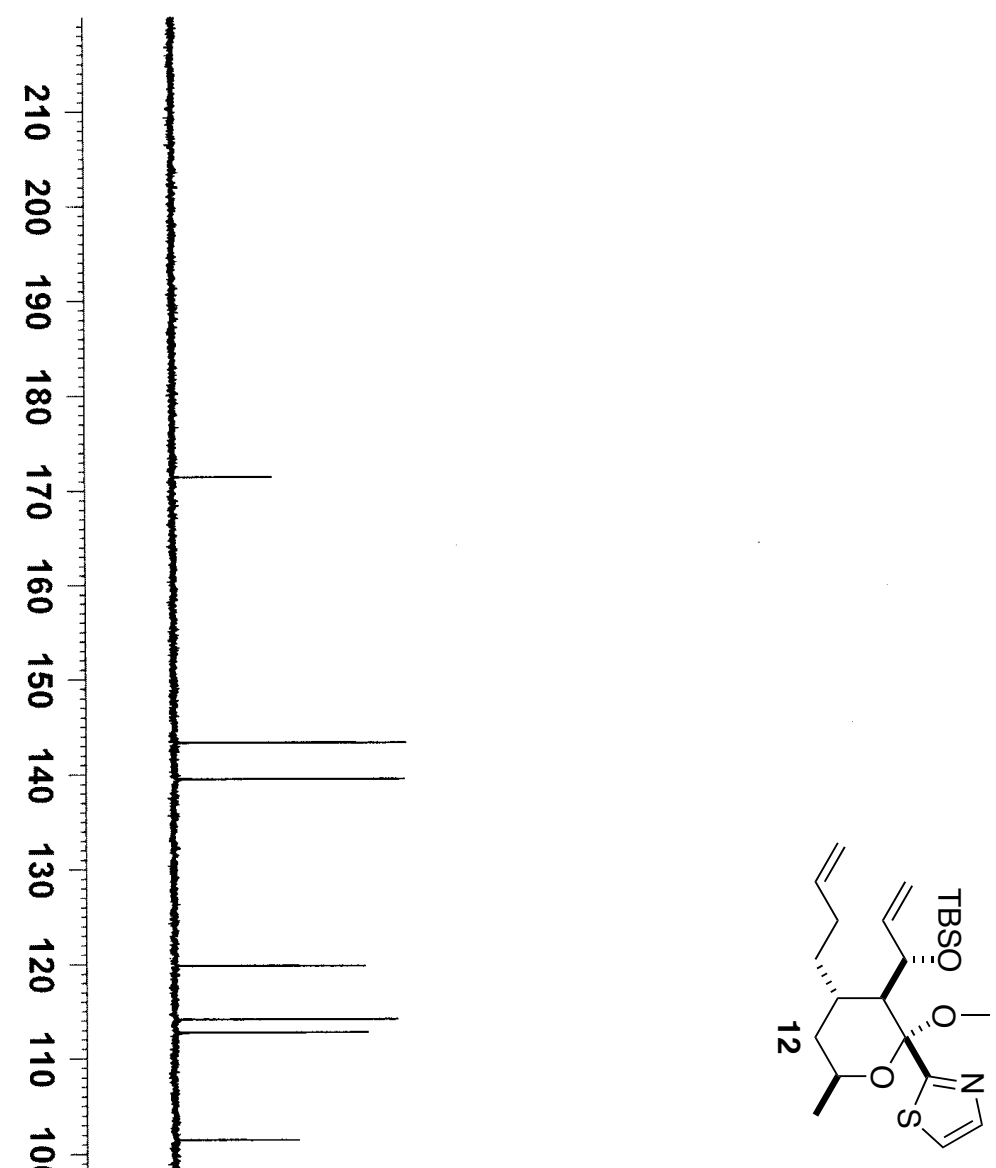

119.87

\&

ป

용

g응

음

$\omega$

$\omega$

ก

$\overrightarrow{0}$

量

寿

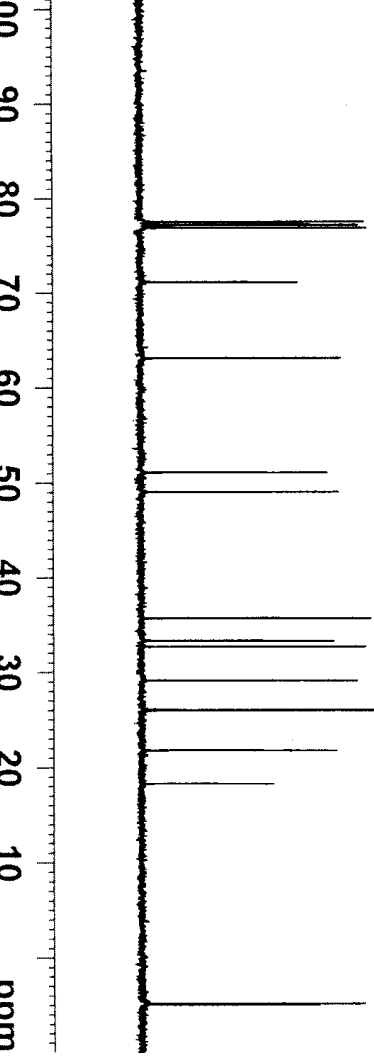

$-77.55$

77.23
-76.92

$-63.12$

$-51.13$

$=49.07$

$-35.71$

$-33.35$

32.72

29.15

$-26.05$

$-21.82$

$-18.31$

-4.83
$<-4.95$ 


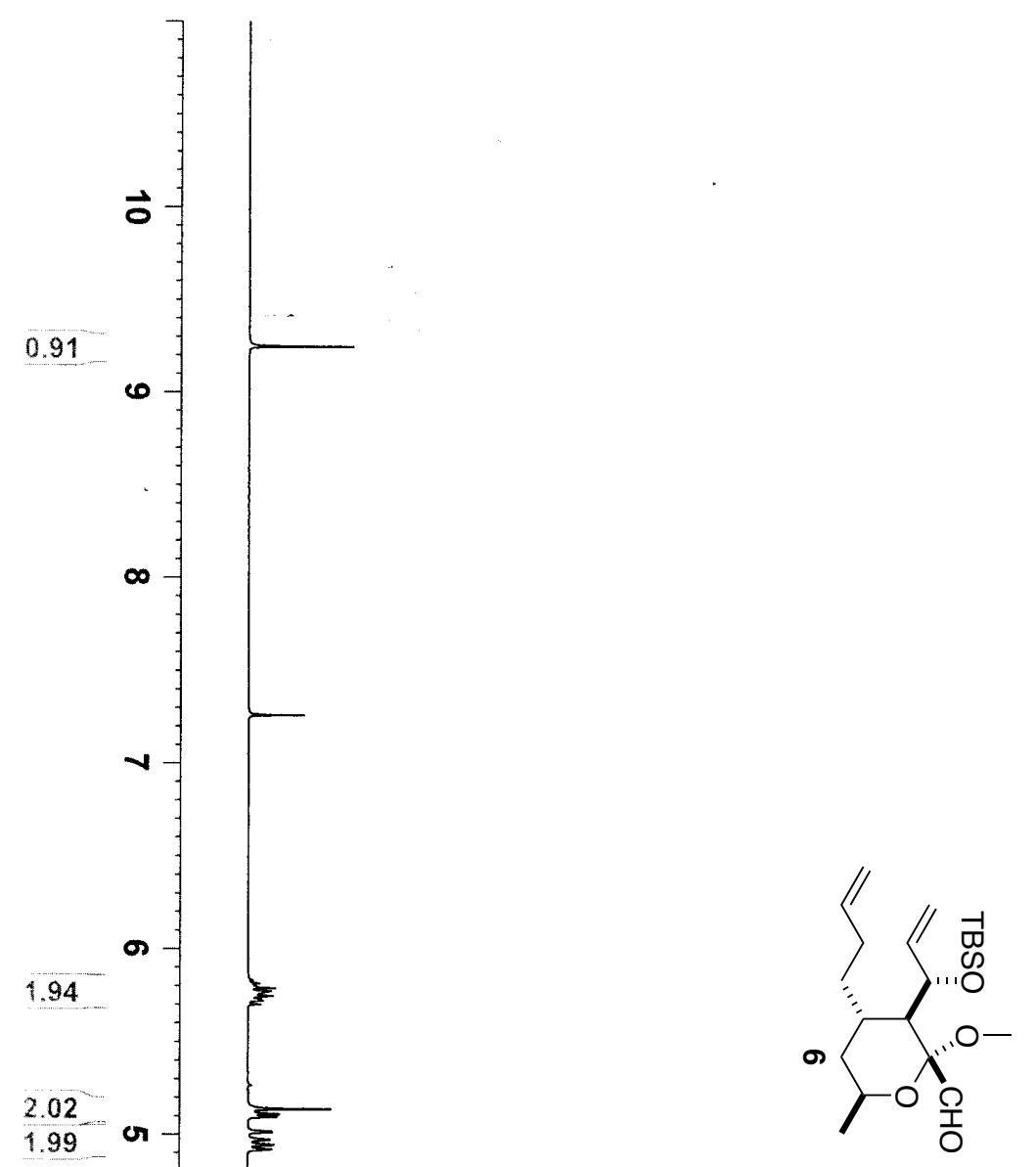

7.260

5.832

$-5.815$

-5.806
-5.798

-5.789
-5.772

$-5.772$

$-5.765$

5.746

$-5.730$

5.721

5.701

5.139

5.111
5.097

5.097

5.018

5.014

4.975

4.971

4.921

4.233
4.213

$r^{4.213}$

3.932

3.916

3.909

3.901
-3.894

3.887
-3.880

- 3.880

$-3.872$

3.865

3.857

[3.849

3.127

2.072

2.053

2.036

2.019
-1.896

1.896

-1.892

$-1.873$

1.873
-1.853

$-1.838$

-1.827
-1.814

$L_{1.795}$

1.778
1.639

1.611

1.594
1.587

1.580

1.574

1.561

$-1.552$

$-1.543$

$-1.523$

응

$-1.509$ 


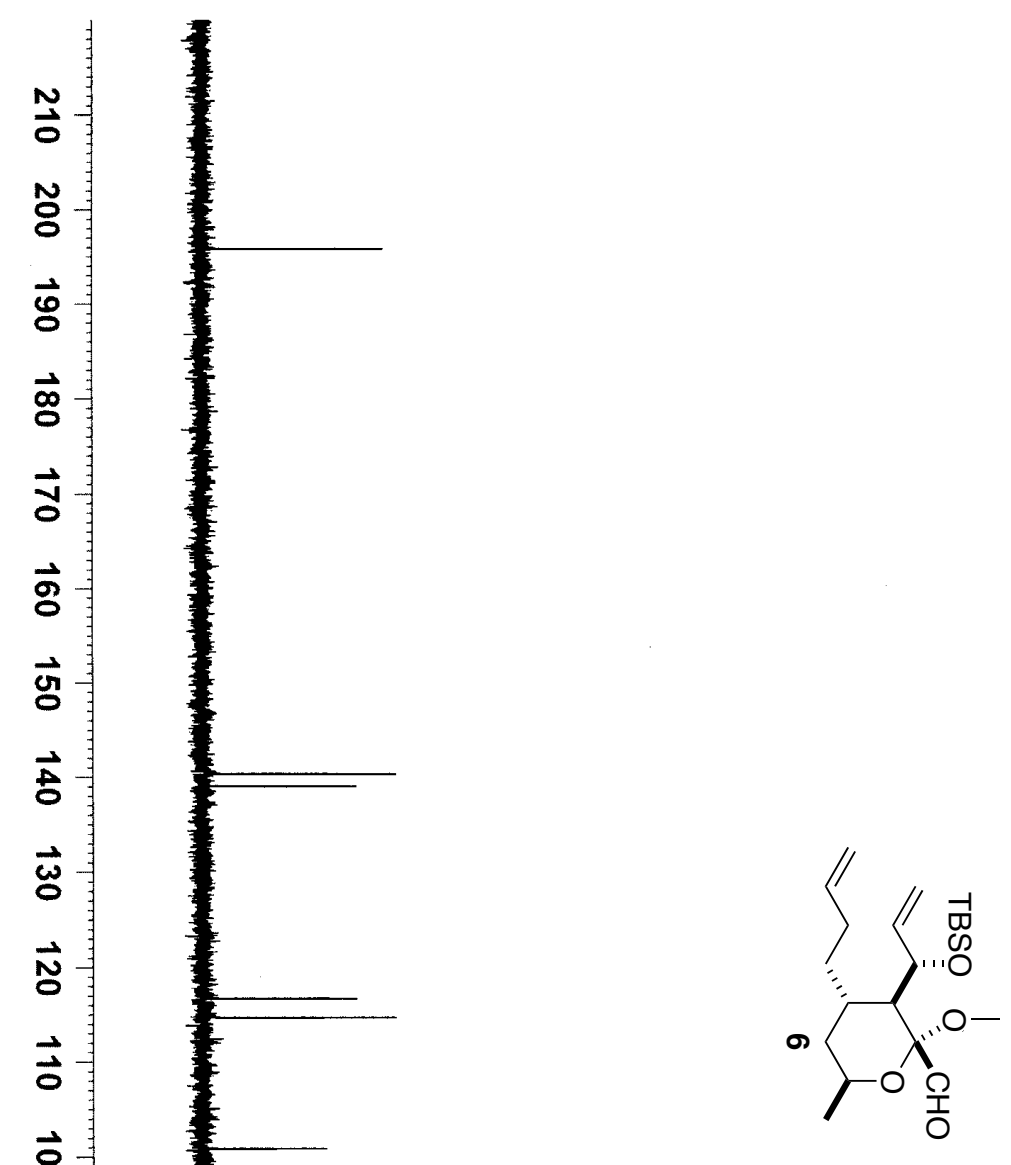

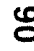

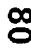

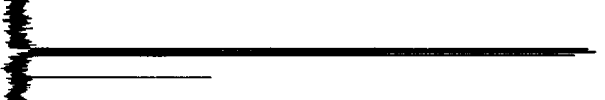

$\begin{array}{r}77.55 \\ -77.23 \\ \hline\end{array}$

77.23

76.91
-74.58

ป

8

or

49.72
-48.68

of

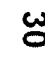

$\omega$ 青坚

品是

$-63.07$

33.73
32.94

33.73
-32.94

31.57

$-26.30$

$-21.81$

$-18.46$

$\times \begin{array}{r}-3.37 \\ -4.09\end{array}$ 

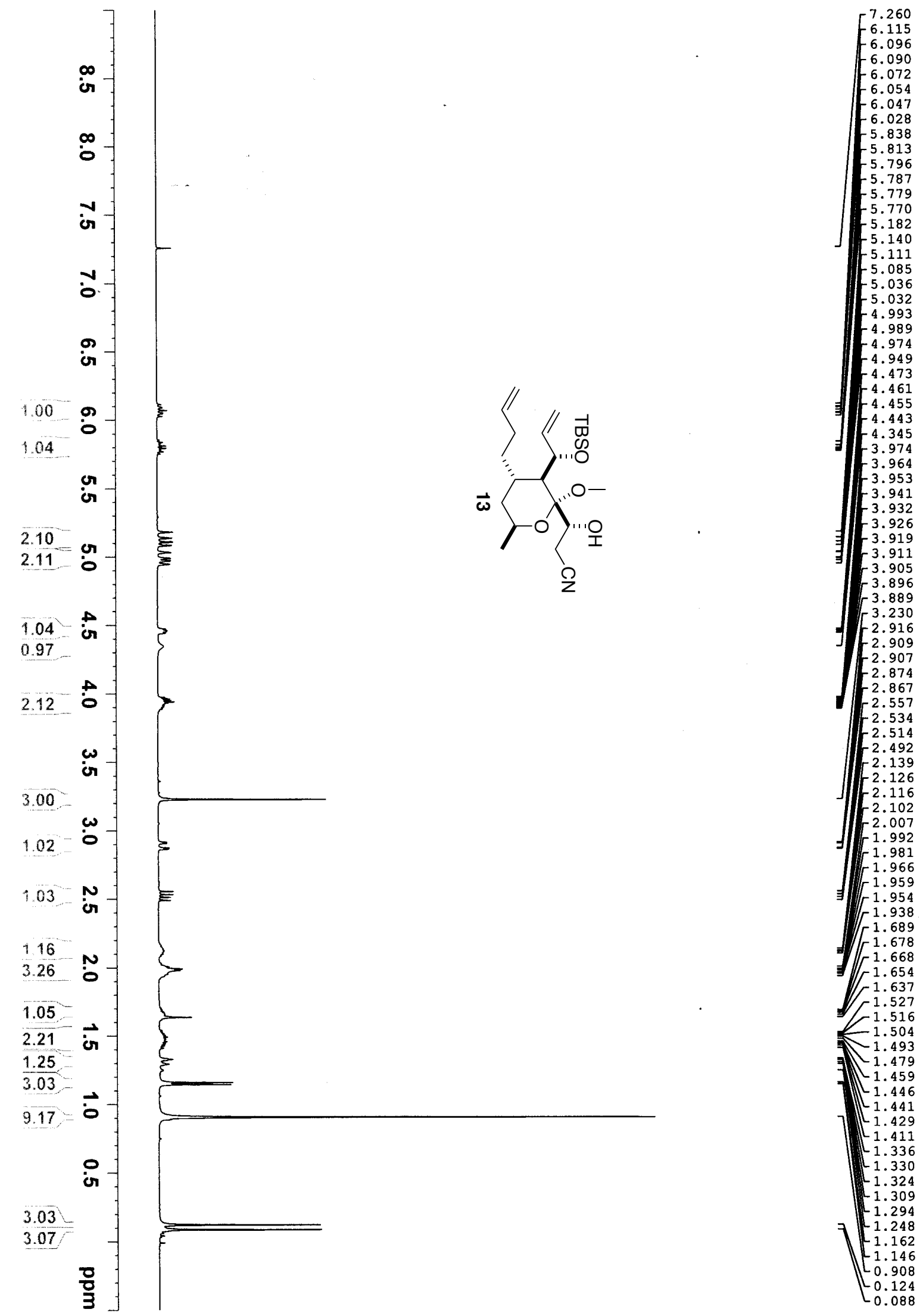

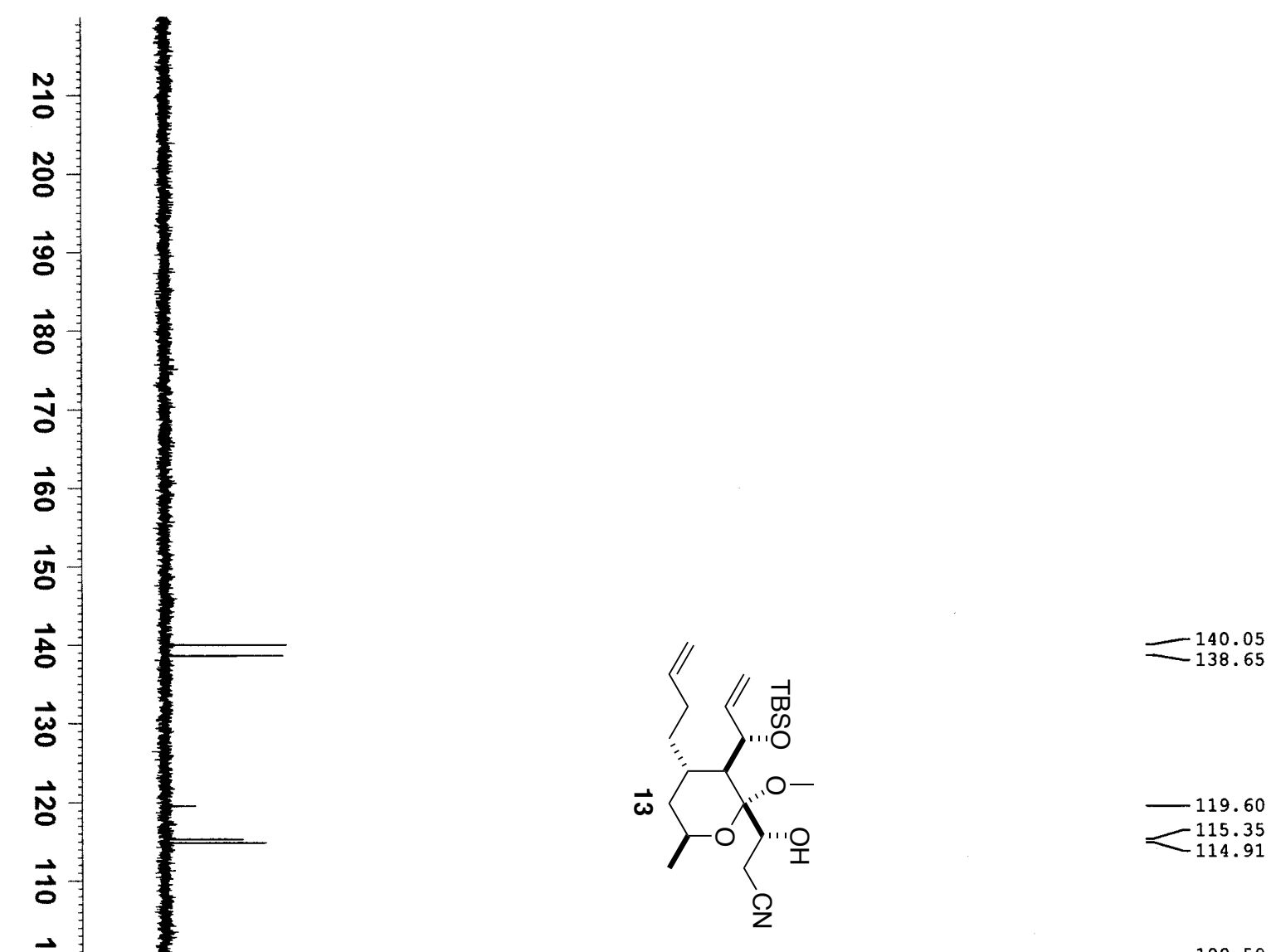

$-119.60$

$-115.35$

$-114.91$

$-100.59$

$\ddot{\circ}$

ळ

๖े

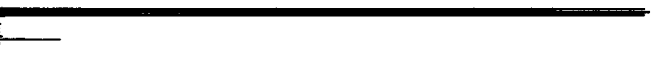

$\begin{array}{r}77.55 \\ -77.23 \\ \hline\end{array}$

$\begin{array}{r}77.55 \\ -76.91 \\ \hline\end{array}$

$-74.35$

$-71.34$

$-64.93$

8

or

年

$-49.58$

$-44.67$

a

๗ั

ก

$\overrightarrow{0}$

品

(1)

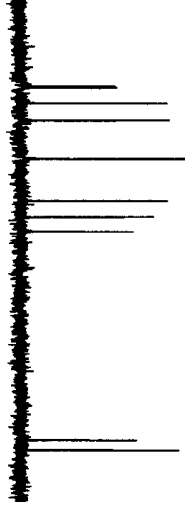

$\begin{array}{r}33.70 \\ \hline 33.55\end{array}$

33.55
-31.93

$-30.13$

$-26.09$

$-21.64$

18.38
-18.38

$-3.54$ 

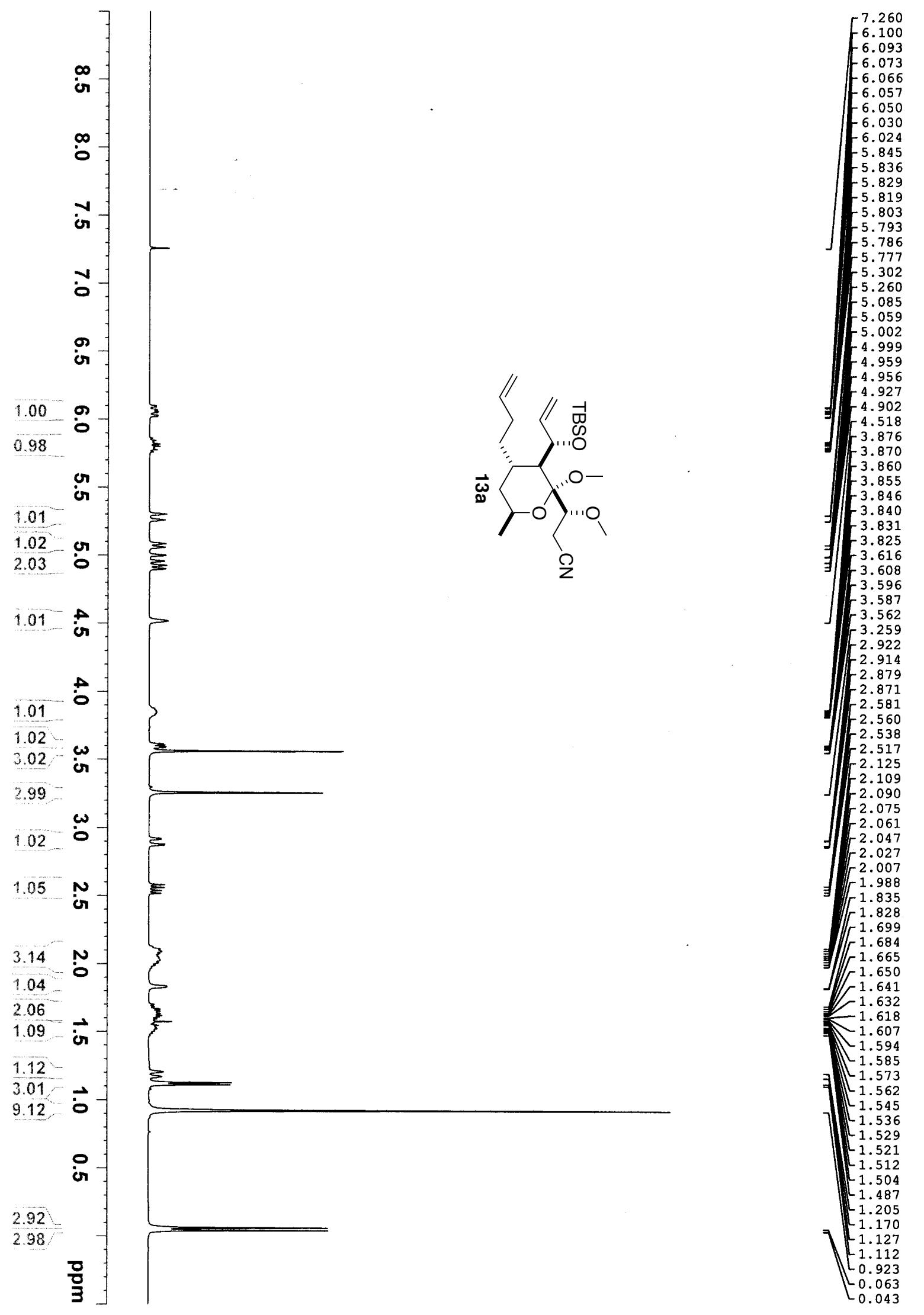


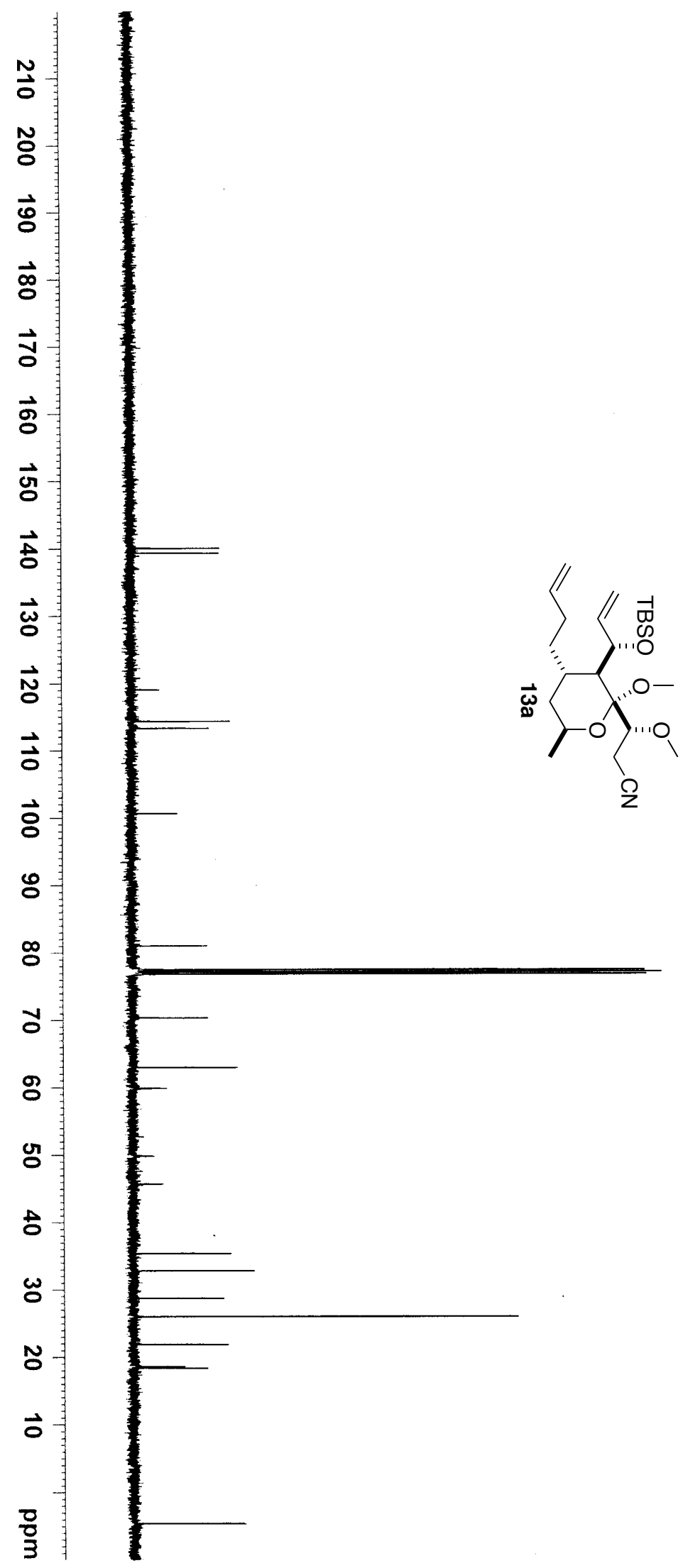

$-119.09$

$-81.07$ -77.55
-77.23 $-76.91$

$-35.42$ $-32.87$ $-32.84$ 28.77

$-21.92$

$-18.63$

$-18.39$

-4.53
--4.59 

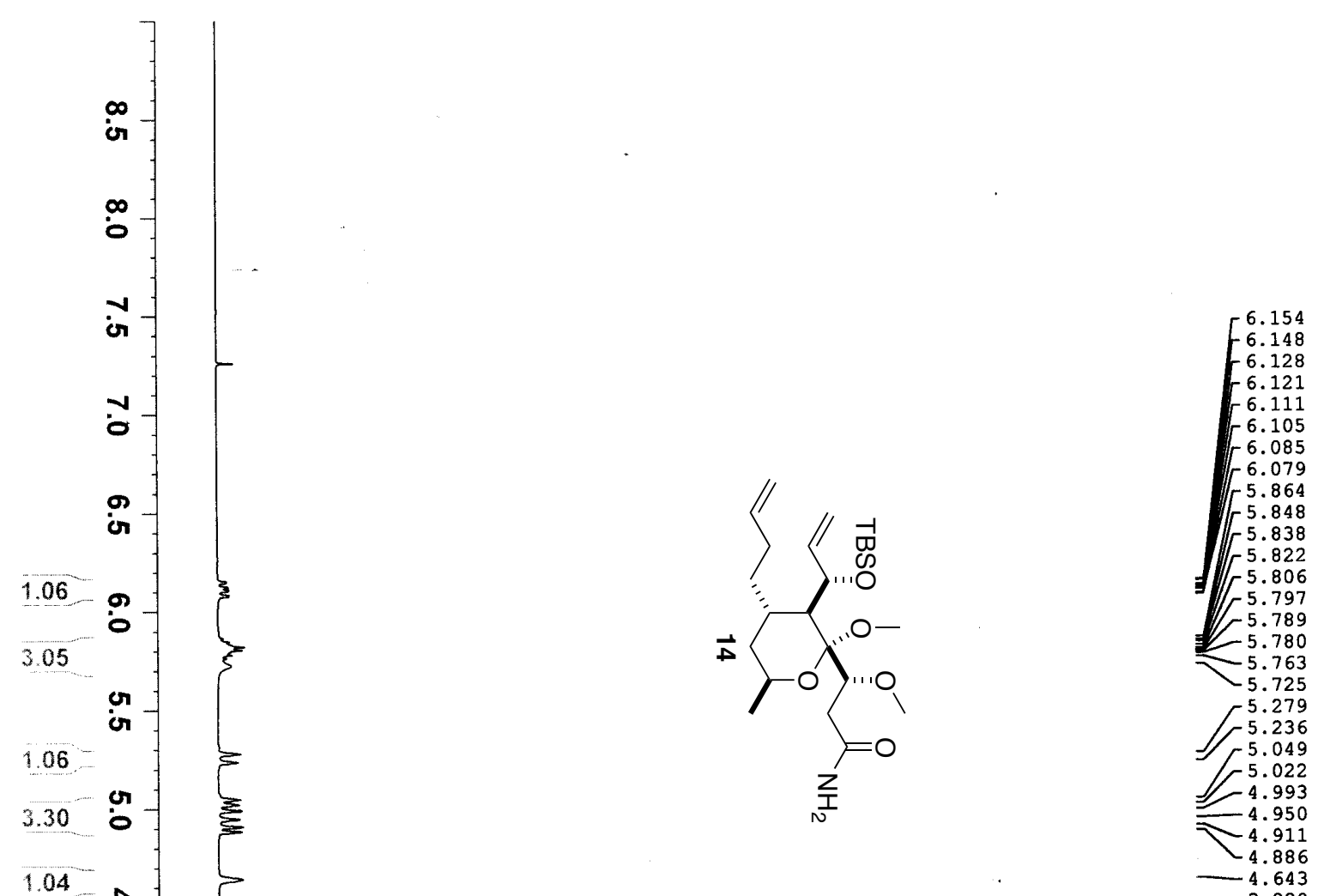

3.820
0

3.820
-3.797

3.797

3.776
3.769

3.769

3.467

3.198

2.804

$-2.798$

2.767
-2.761

2.761
2.346

2.346

$\left[\begin{array}{r}2.324 \\ -2.308\end{array}\right.$

[ 2.308

2.287

J $\left[\begin{array}{l}2.109 \\ 2.093\end{array}\right.$

2.074

2.059

2.044

J 2.035

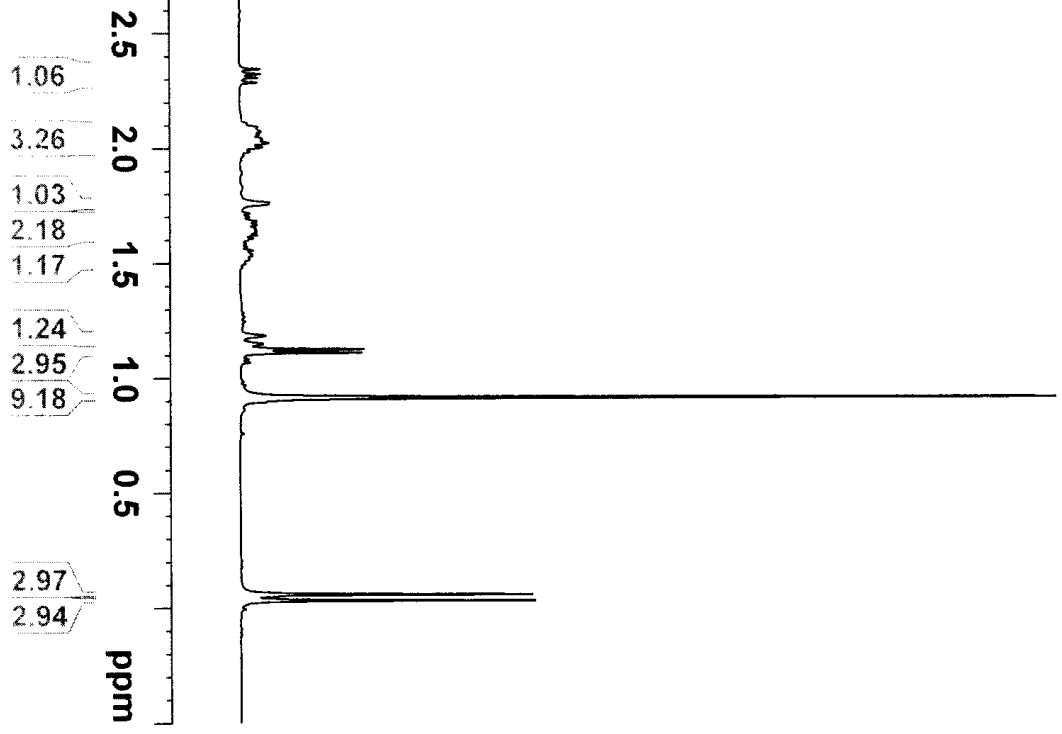

2.023
-2.005

$-1.986$

1.764

1.757

1.717
1.701

1.701
1.682

$\left[\begin{array}{l}1.682 \\ 1.667\end{array}\right.$

1.652

1.643

1.634

1.622

1.608
-1.600

1.585
-1.572

$-1.572$

1.556

$-1.540$

$-1.531$

$-1.522$

$-1.515$

-1.507
-1.498

1.498
1.187 


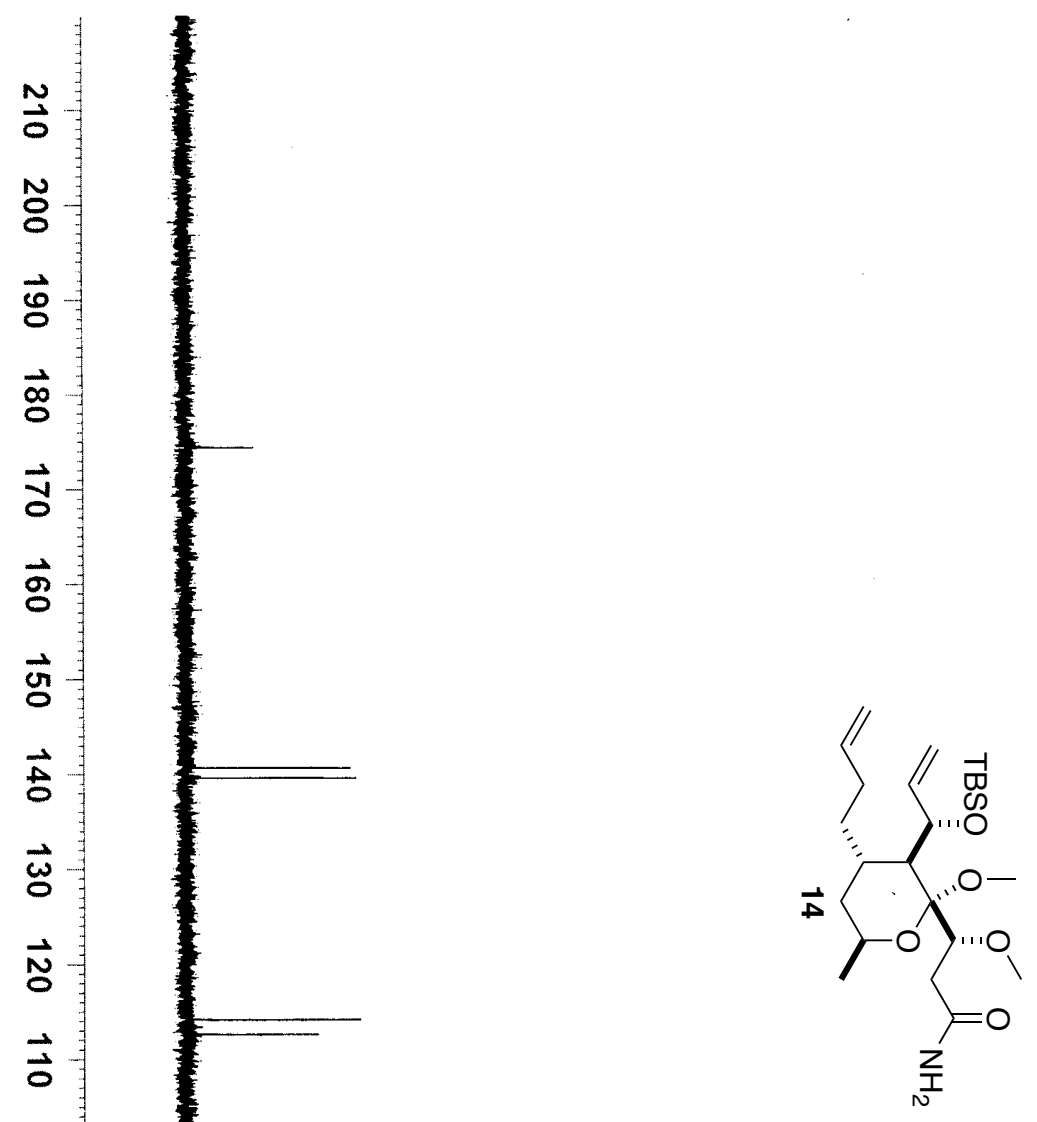

항

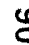

$\stackrel{\infty}{\circ}$

o

용

응

t

$\omega$

을

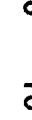

猋

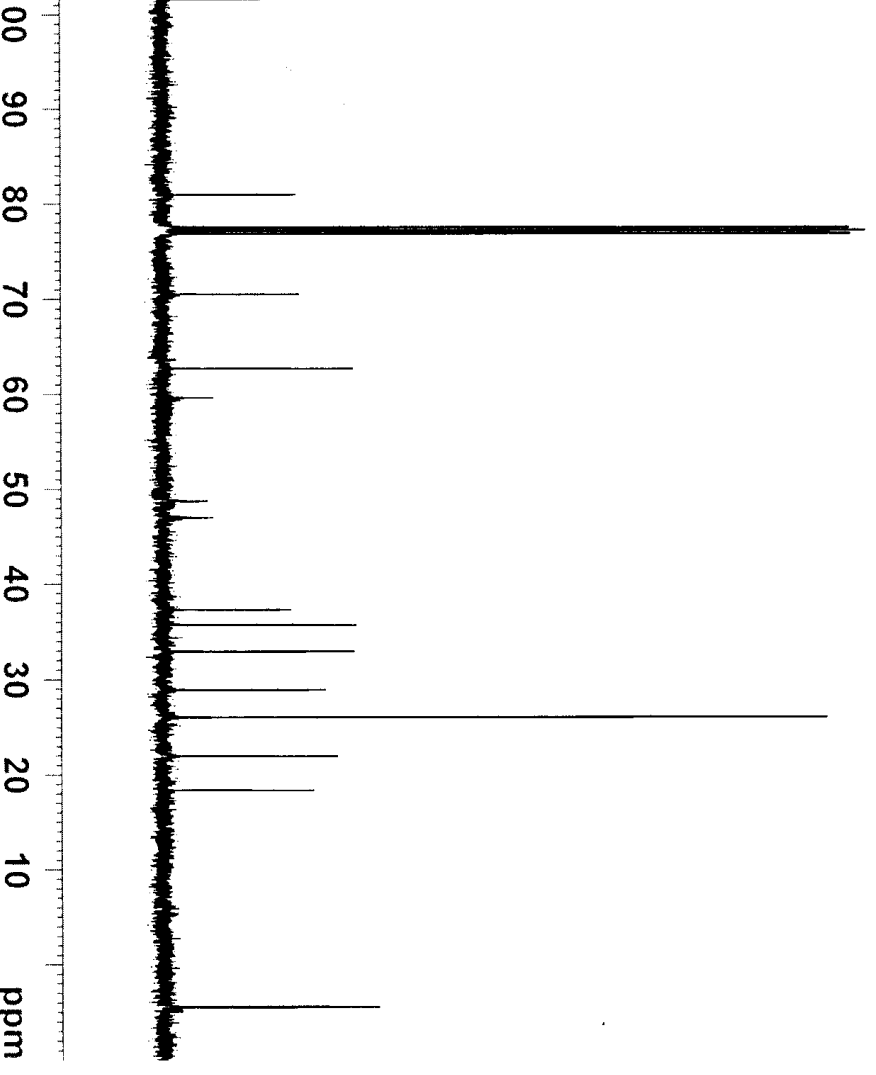

-140.68
-139.59

-80.99
-77.55

-77.55
-77.23

76.91
-70.55

$-70.55$

$-62.73$

$-59.66$

-48.79
$-\quad 47.03$

$\begin{array}{r}37.32 \\ -35.73 \\ \hline\end{array}$

32.97

$\begin{array}{r}32.97 \\ -32.92 \\ \hline\end{array}$

38.94
-

$-26.07$

$-21.99$

$-18.38$

-4.40
-4.47 

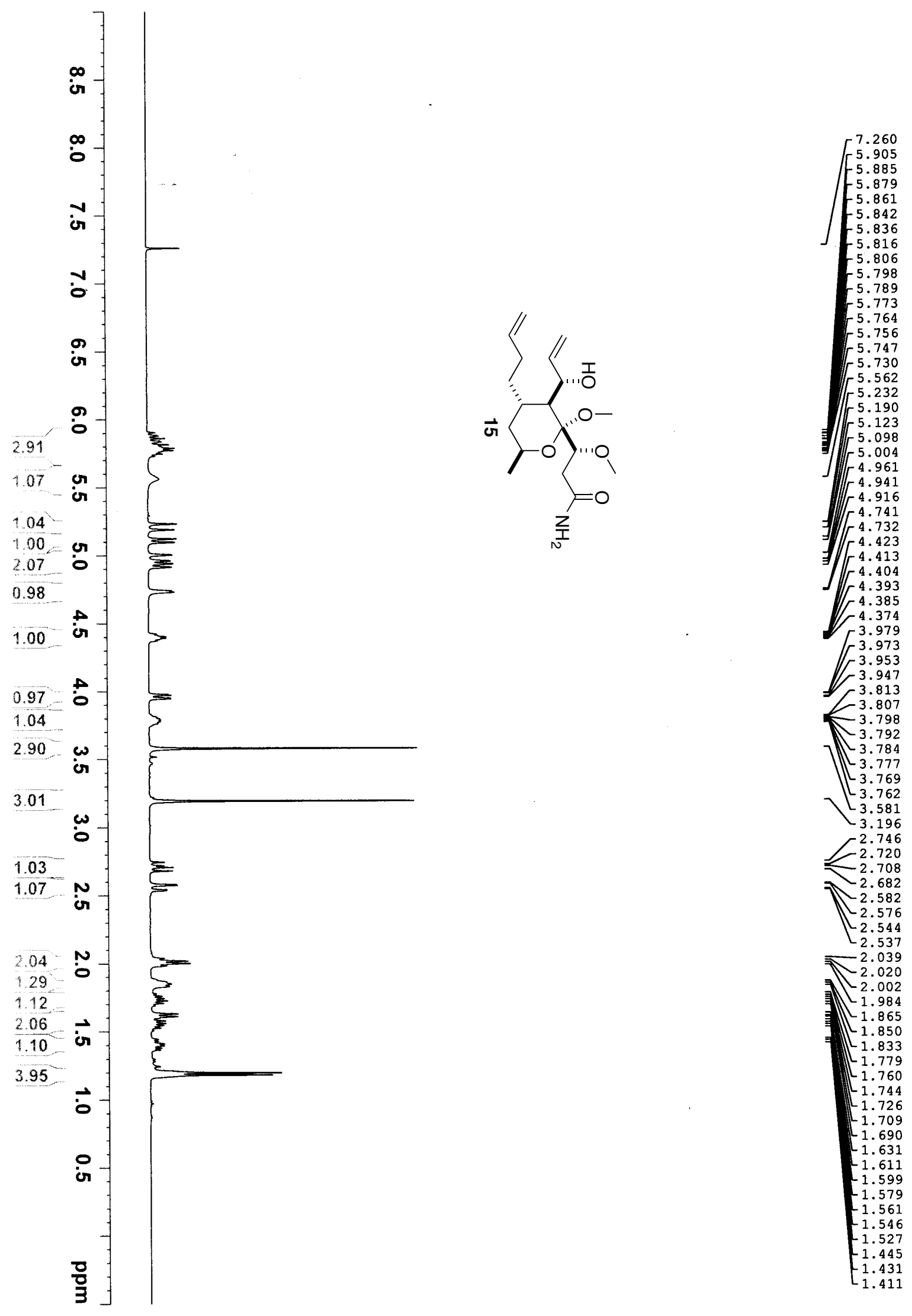


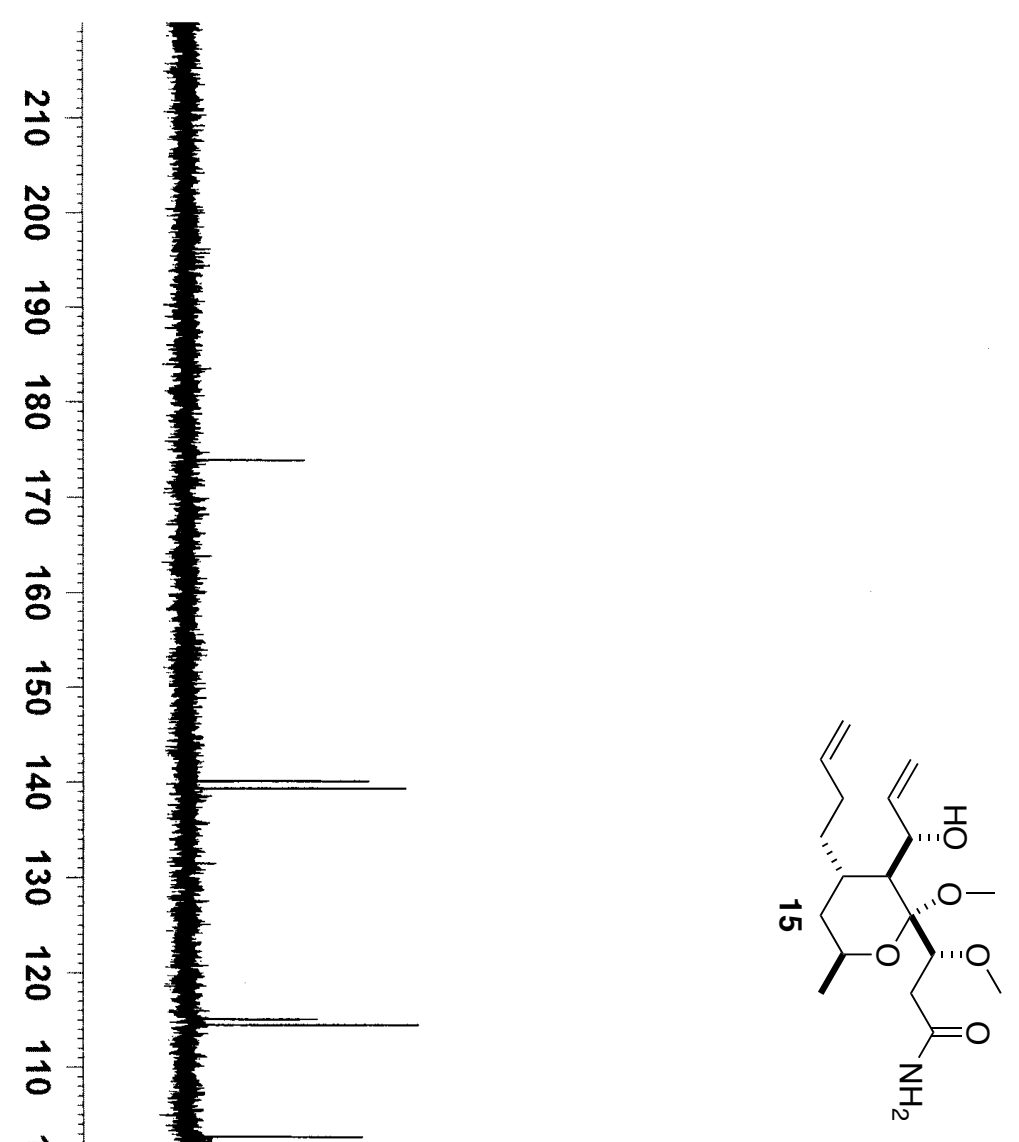

$\overrightarrow{\mathrm{s}}$

8

ø

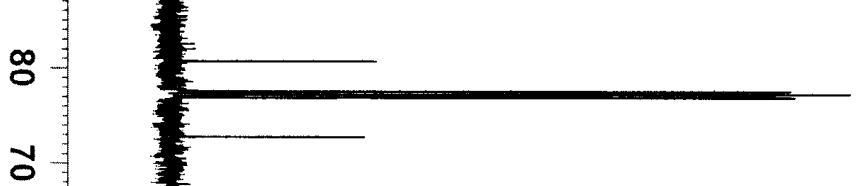

o

8

잉

o

๘

ก

$\overrightarrow{0}$

总 

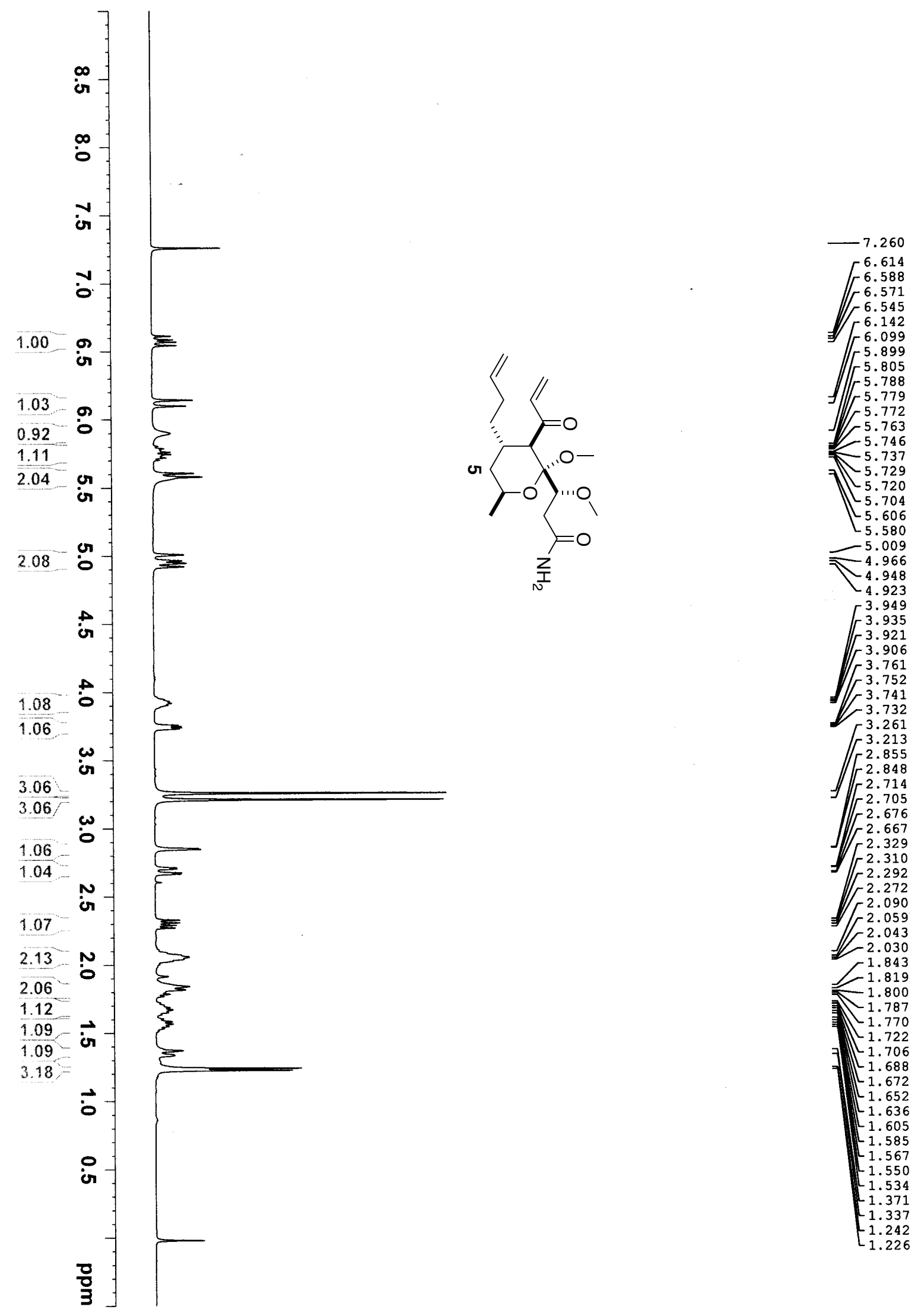


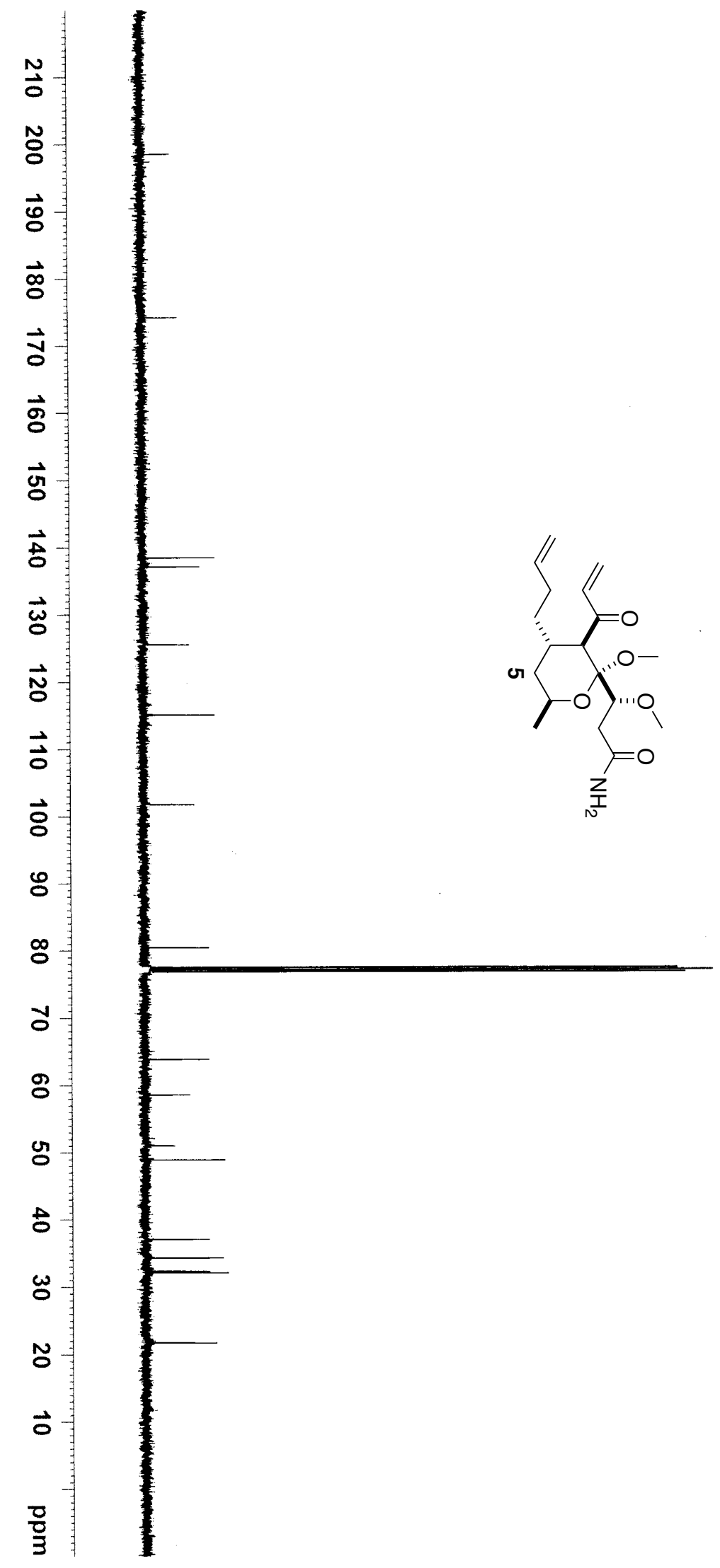

$-198.49$

$-174.19$

$-138.48$

$-125.56$

$-115.07$

$-101.81$

$-80.48$

77.55

77.23

$-63.87$

$-58.61$

-51.07
$-\quad 48.95$

$-37.08$

$-34.33$

32.36

$-32.29$

$-32.12$

$-21.77$ 

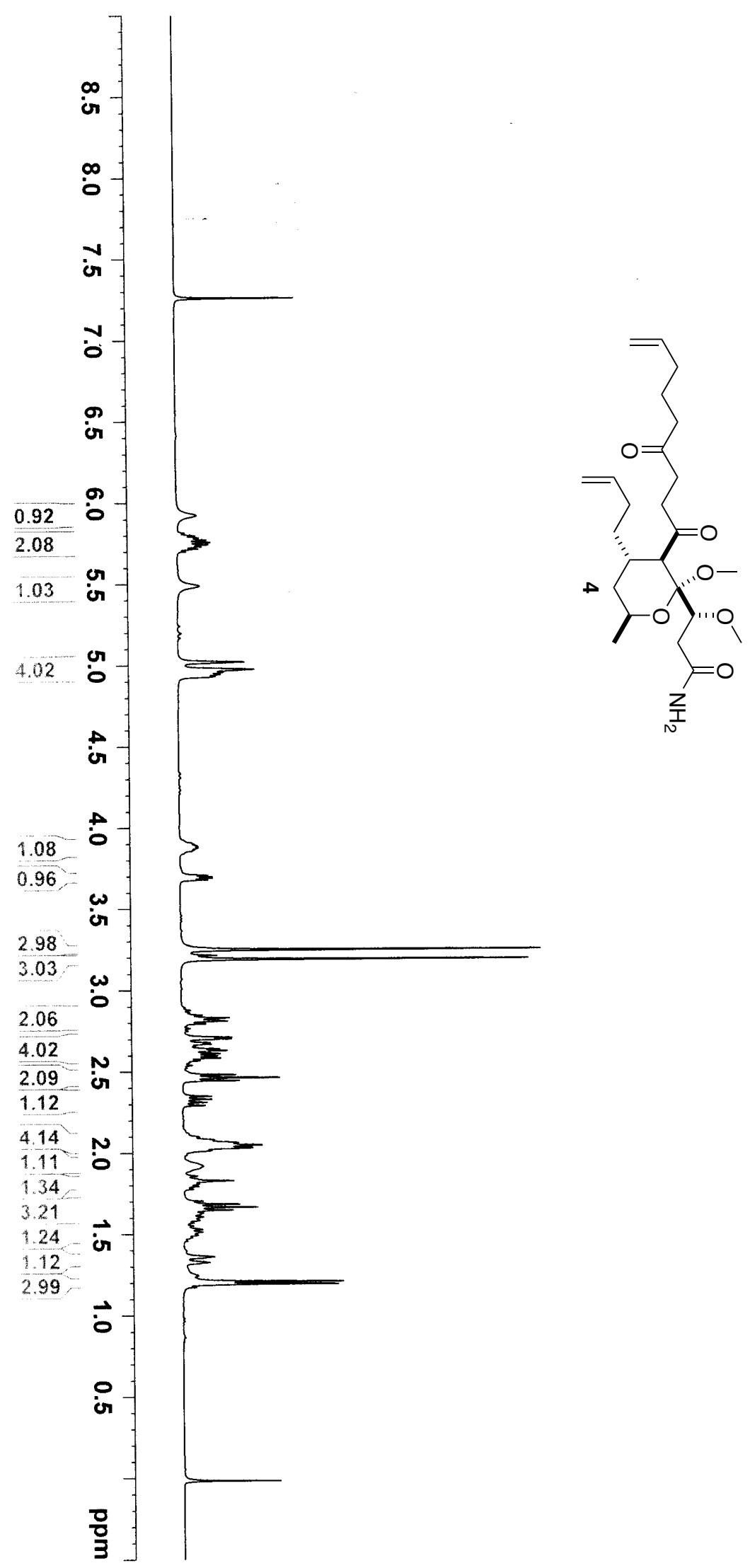

5.924
-5.815
-5.799
-5.786
-5.773
5.750

$-5.760$

$-5.757$

5.743

5.731

5.701

5.488
-5.022

5.022

5.019

$-4.975$

$-4.972$

4.946

4.933

3.901

3.901

$-3.893$

$-3.879$

$-3.873$

$-3.864$

3.864
-3.709
-3.699

3.699

3.699
3.690

3.690

3.680

$\left[\begin{array}{r}3.250 \\ 3.191 \\ -2.849 \\ -2.833\end{array}\right.$

2.849
2.833

$\left[\begin{array}{r}2.833 \\ -2.812 \\ 2.796\end{array}\right.$

2.796

$-2.713$

$-2.703$

2.680

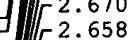

$\Gamma^{2.643}$

2.632

2.614

2.594
2.584

2.569

- 2.483

2.465
2.446

2.350
-2.351

$\left[\begin{array}{r}2.350 \\ 2.331 \\ 2.313\end{array}\right.$

$-2.313$

2.294
2.097

2.080

2.080
2.063

2.049

2.032

2.014

1.917

$-1.858$

$-1.811$

1.794

1.777

1.704

$-1.685$

$-1.667$

$-1.648$

$-1.648$

$-1.630$

-1.623
-1.606

-1.606
1.592 


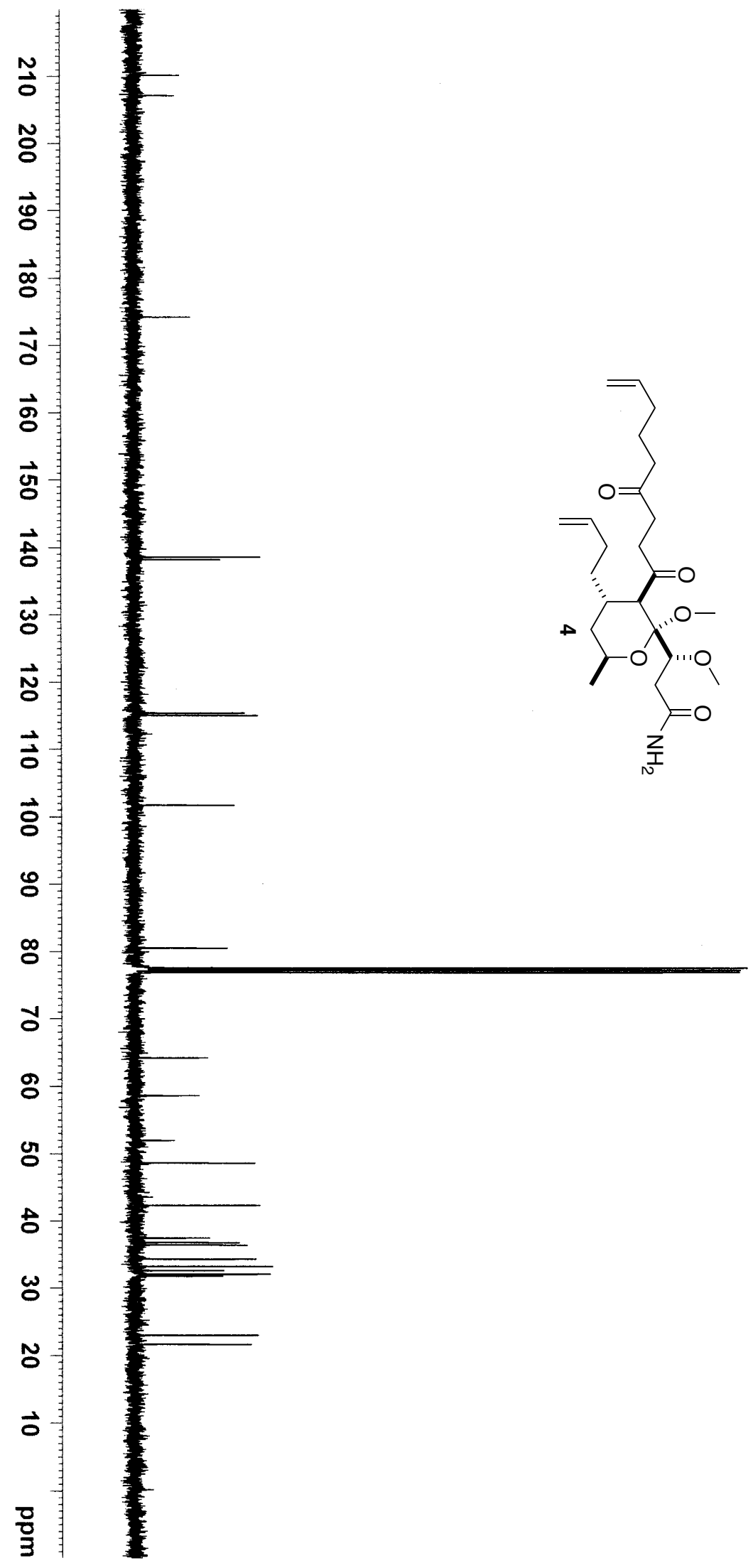

-51.98
-48.63

$-42.32$

37.45

- 36.40

$-34.31$

$-33.25$

32.61
-32.08

$-32.08$

31.79

$-23.00$

21.67 

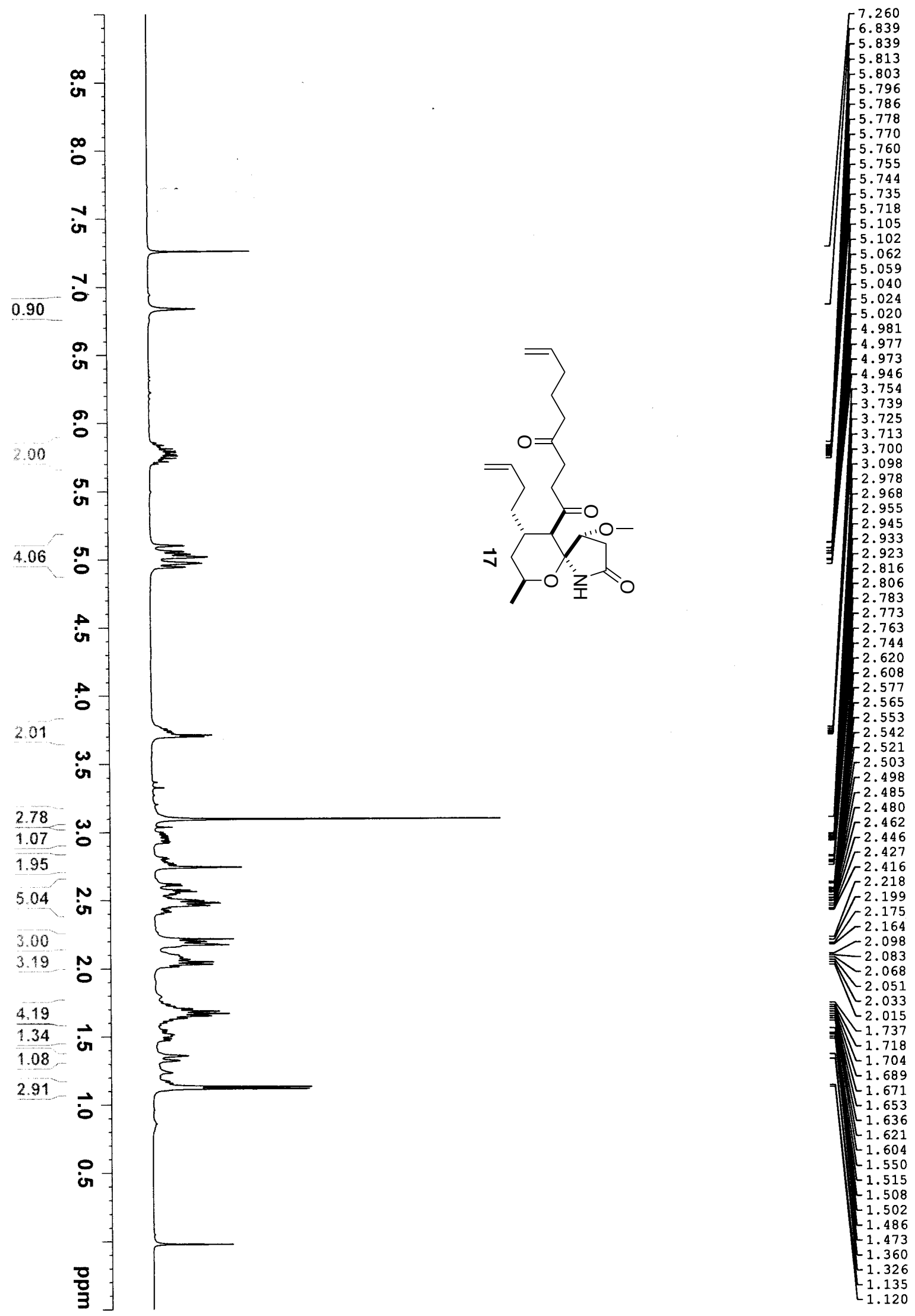


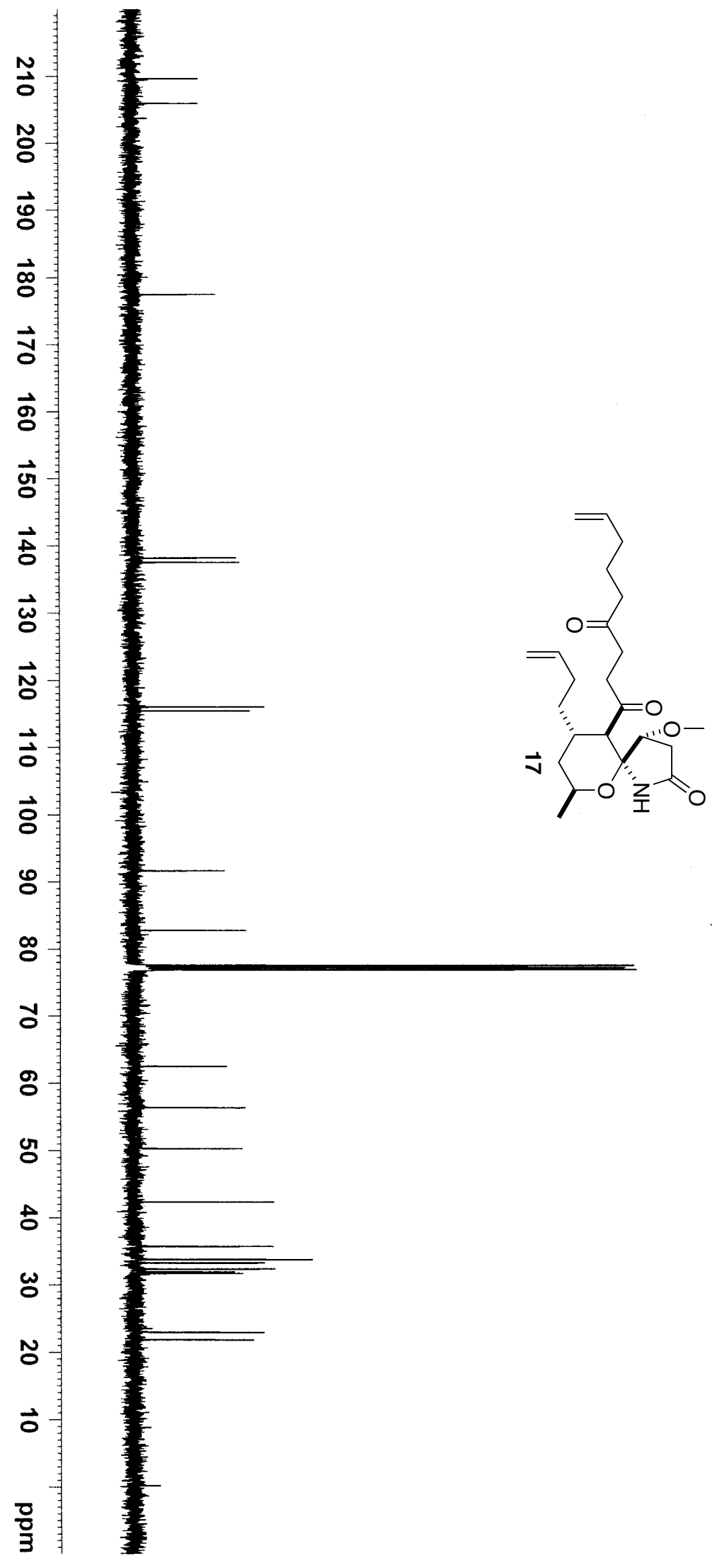




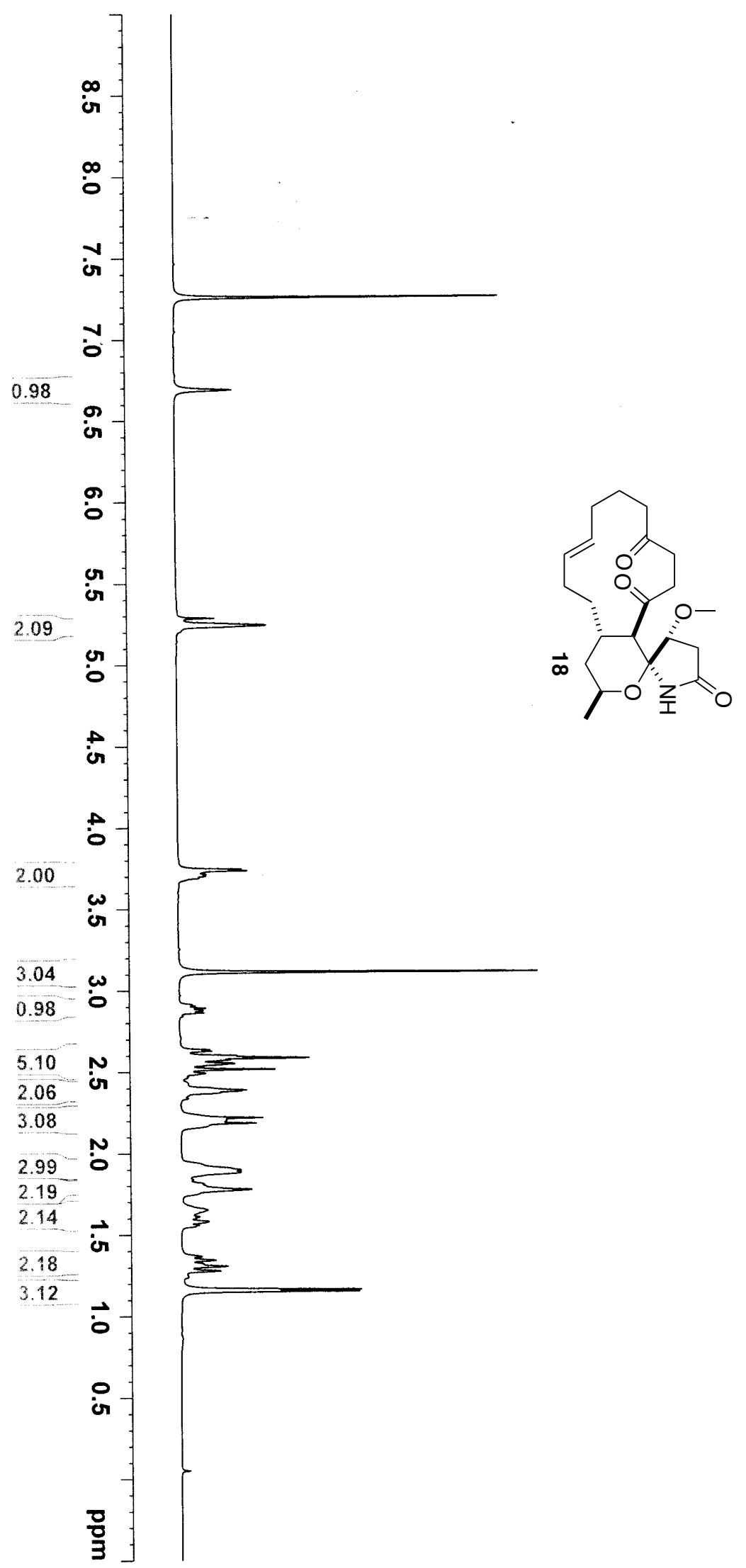
3.738
-3.714 3.704
-3.693 3.693 $-3.116$ 2.916 $-2.908$ $-2.893$ 2.880 2.876 . 864 $\left[\begin{array}{r}2.8638 \\ 2.638\end{array}\right.$ 2.628 ] -2.601 2.588 2.575
-2.553 2.553
2.519 2.519
-2.494 $-2.392$ 2.392
-2.387 $-2.377$ $-2.223$ $-2.208$ 2.208
-2.189 2.189
-1.900 1.800
1.887 1.887
1.839 $\left[\begin{array}{l}1.839 \\ 1.814\end{array}\right.$ $\left[\begin{array}{r}1.814 \\ 1.801\end{array}\right.$ 3) $\left[\begin{array}{l}1.801 \\ 1.780\end{array}\right.$ $\left[\begin{array}{l}1.654 \\ 1.611\end{array}\right.$ 1.611 $\left[\begin{array}{r}1.601 \\ 1.582\end{array}\right.$ $\left[\begin{array}{l}1.589 \\ 1.559\end{array}\right.$ 1.551 $-1.368$ 1.345
1.322 $-1.322$ 1.308 $-1.280$ $\left[\begin{array}{r}1.167 \\ 1.155\end{array}\right.$ 


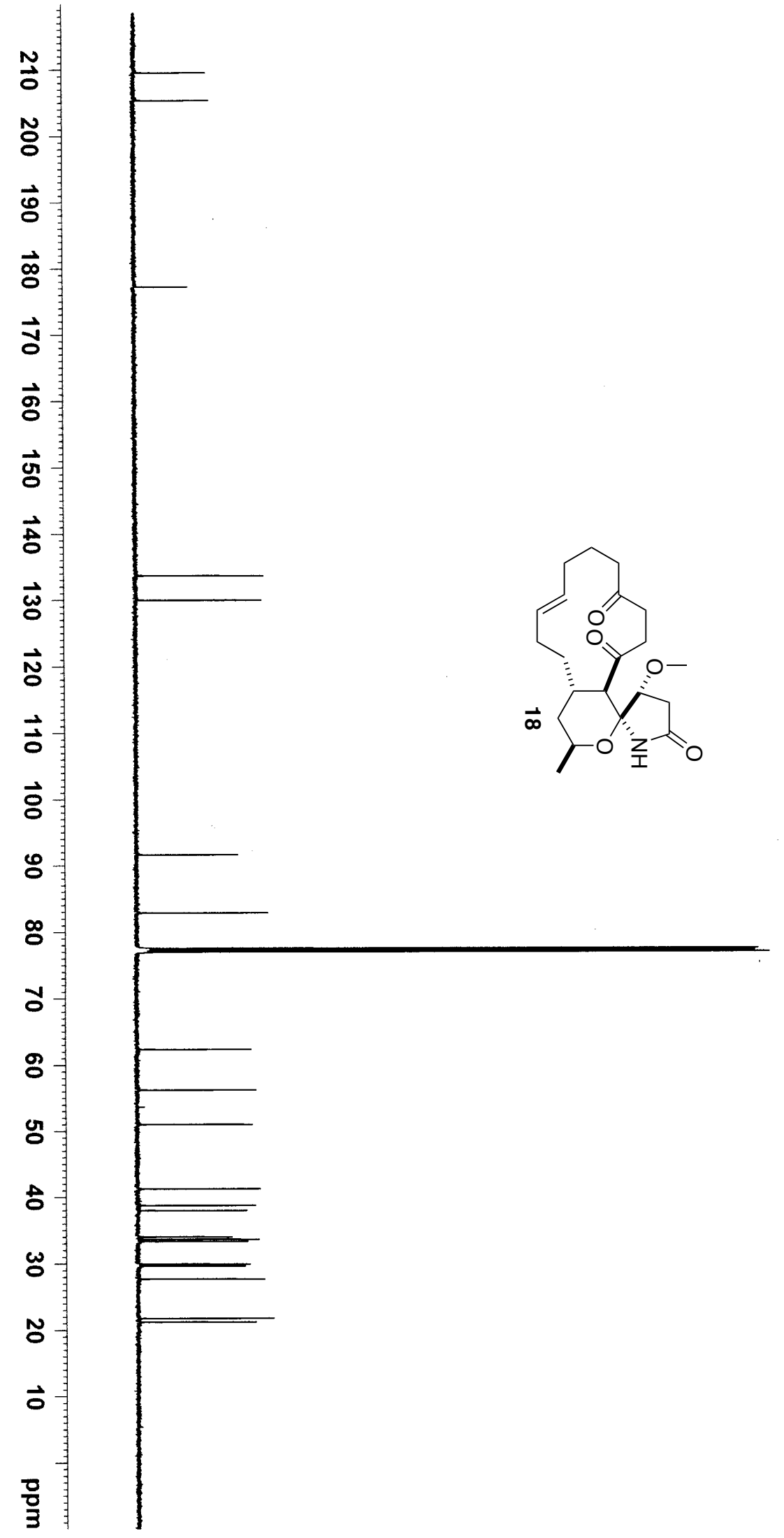

$-209.51$

$-177.18$

$-129.94$

$-91.60$

$-77.48$

77.23
-76.97

$-62.30$

$-56.19$

$-50.98$

$-41.24$

38.75

37.97

$-37.97$

r 34.02

$-33.65$

$-33.34$

$-29.96$

$-29.66$

$-27.70$

21.76
-21.21 


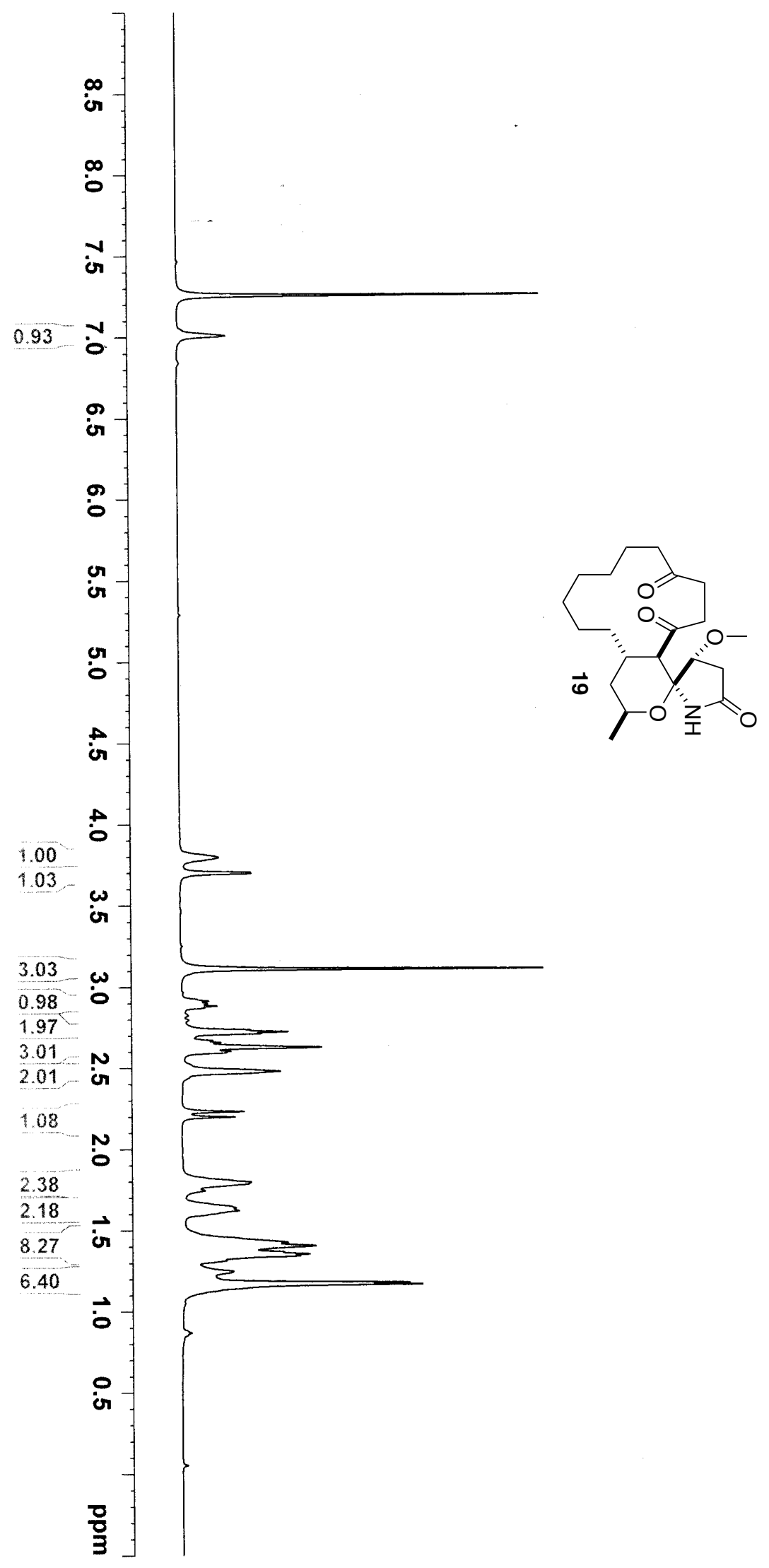

$-7.260$

3.797
3.704

3.704
-3.697

3.697
-3.114

3.114

2.899
2.899

2.885

2.873
-2.736

2.736
2.725

$\begin{array}{r}2.725 \\ \hline\end{array}$

2.714
2.670

2.670
-2.658

2.629

$-2.604$

2.596

2.481
-2.234

2.200

1.797

1.788

$-1.745$

$-1.640$

1.623

1.427
1.406

1.369
1.369

$-1.356$

- 1.345

1.345
1.315

1.249
1.281

$\left[\begin{array}{r}1.181 \\ 1.169\end{array}\right.$ 


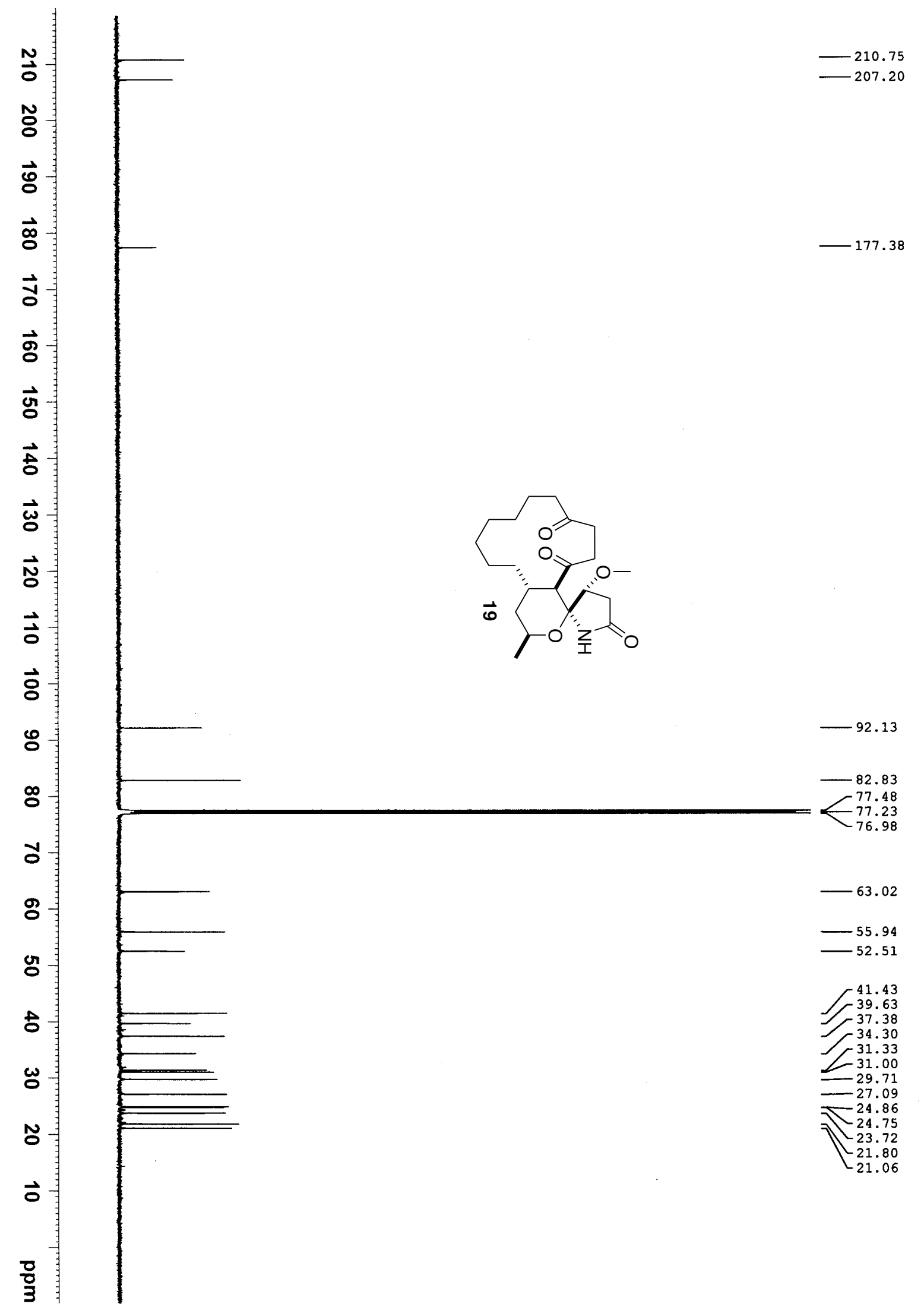



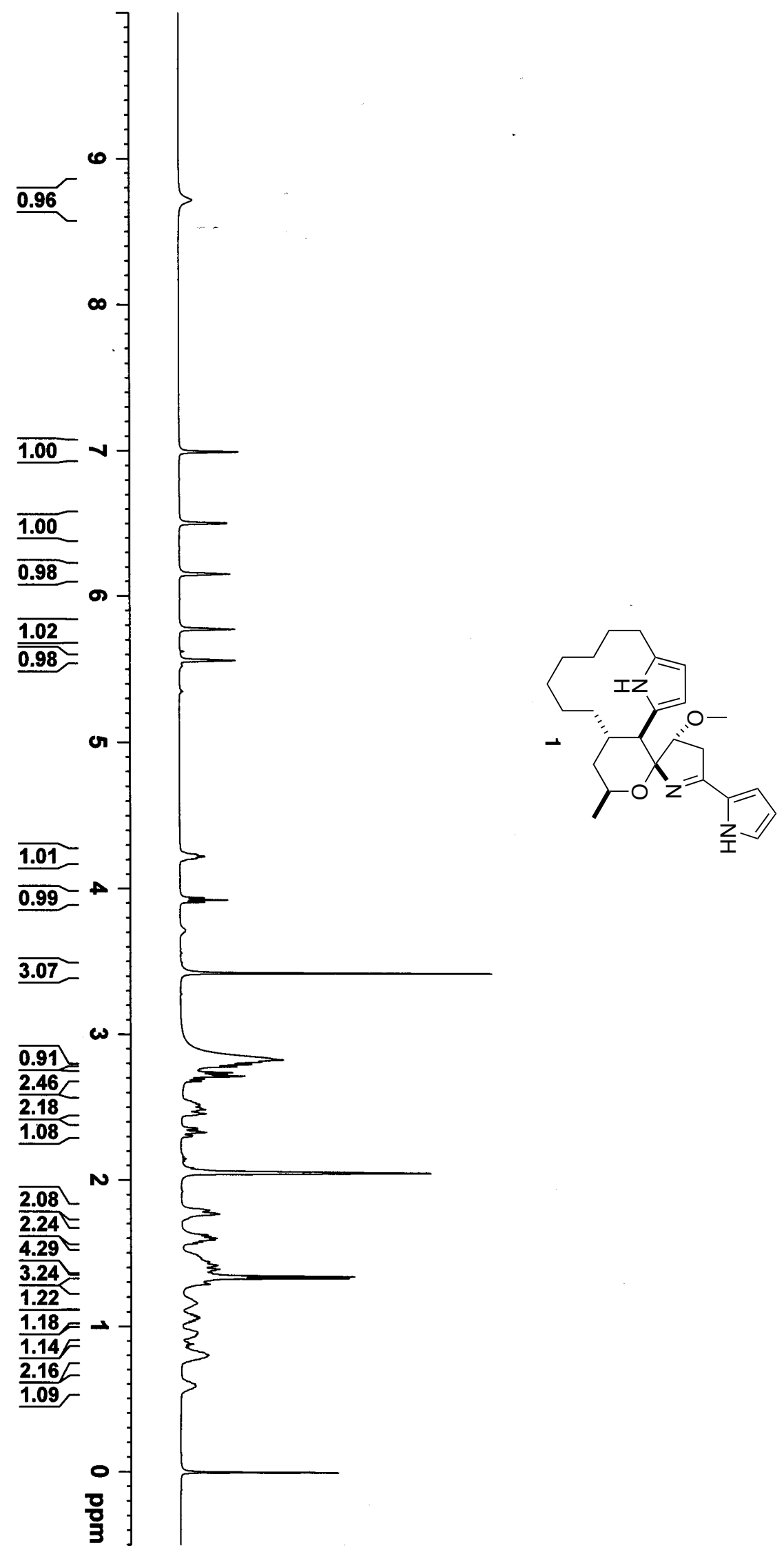

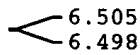

$r^{6.152}$

5.561

-4.233

$-4.211$

$-3.938$

$-3.923$

- 3.907

3.421

2.826

-2.739
-2.726

$-2.726$

$-2.715$

$-2.679$

2.542

$-2.530$

2.521

2.510

2.495

$-2.486$

2.477

.

2.448

2.358

2.352

$-2.335$

.

$\begin{array}{r}2.052 \\ \hline\end{array}$

2.050
-1.794

$-1.788$

1.768

$-1.626$

$-1.615$

1. 591

1.591
1.578
1.566

1.566

1.487

1.477

1.469

1.460

$-1.450$

1.440

$-1.433$

$-1.419$

-1.412
-1.391

1.391
1.384 


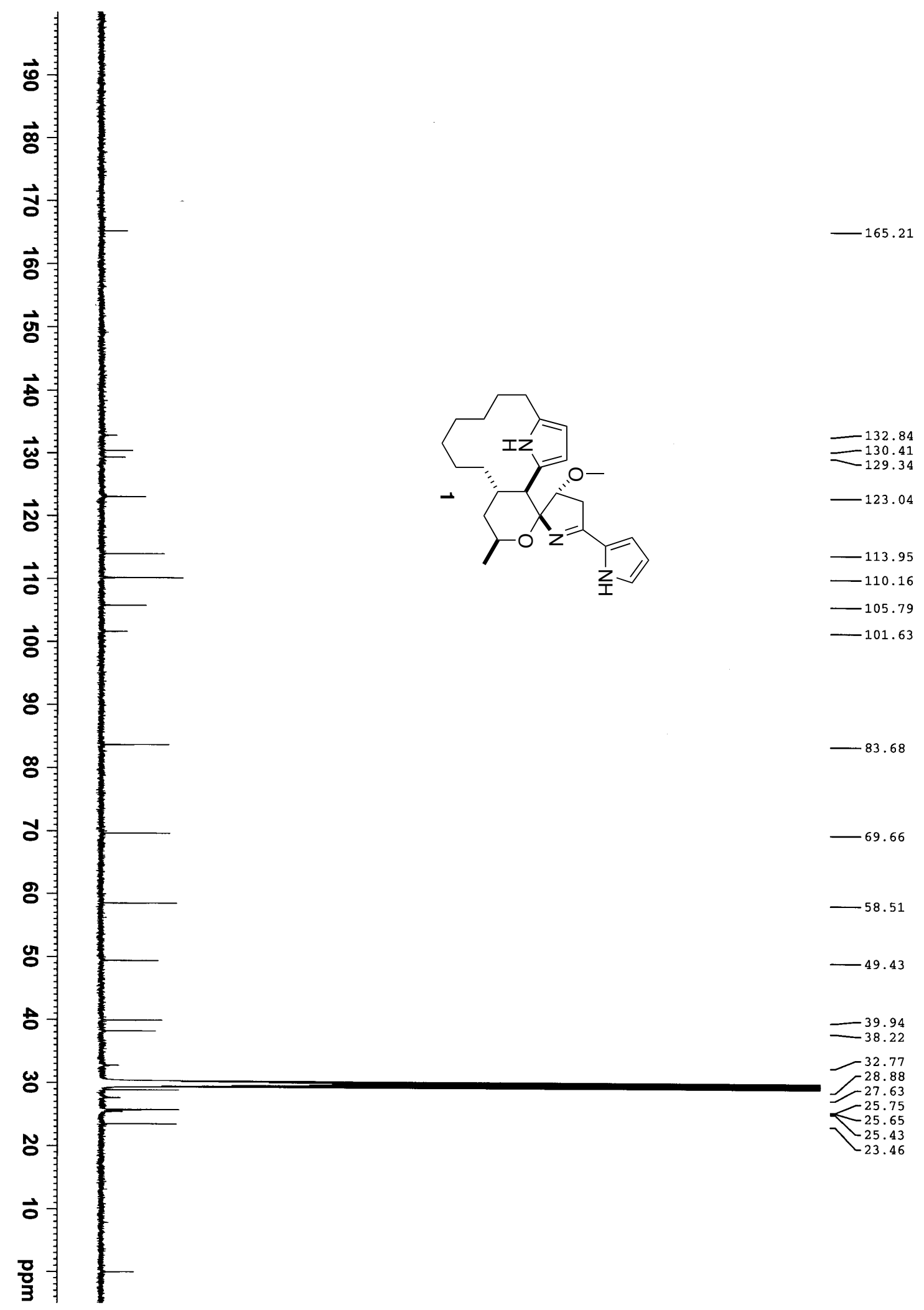




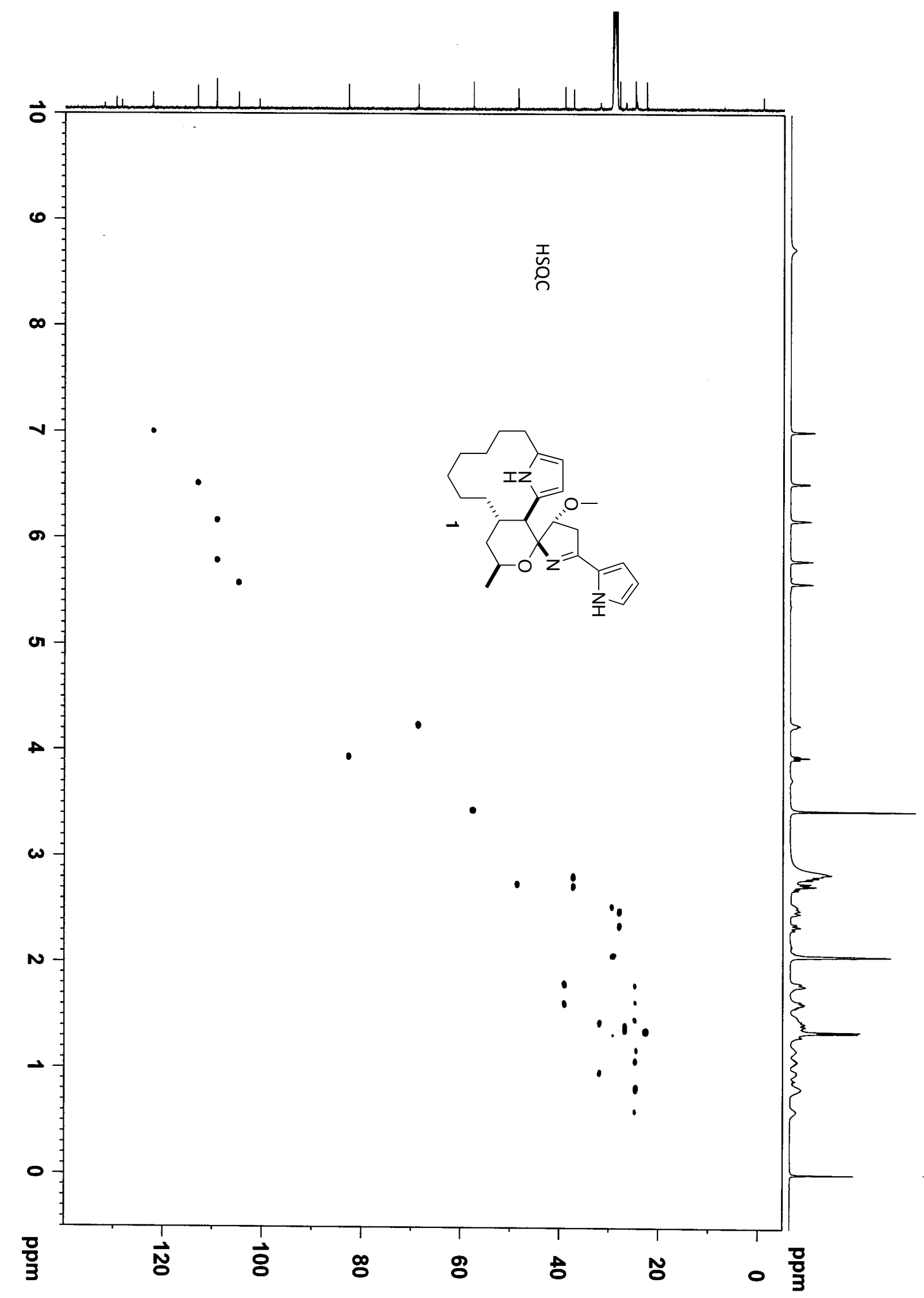

S-118 


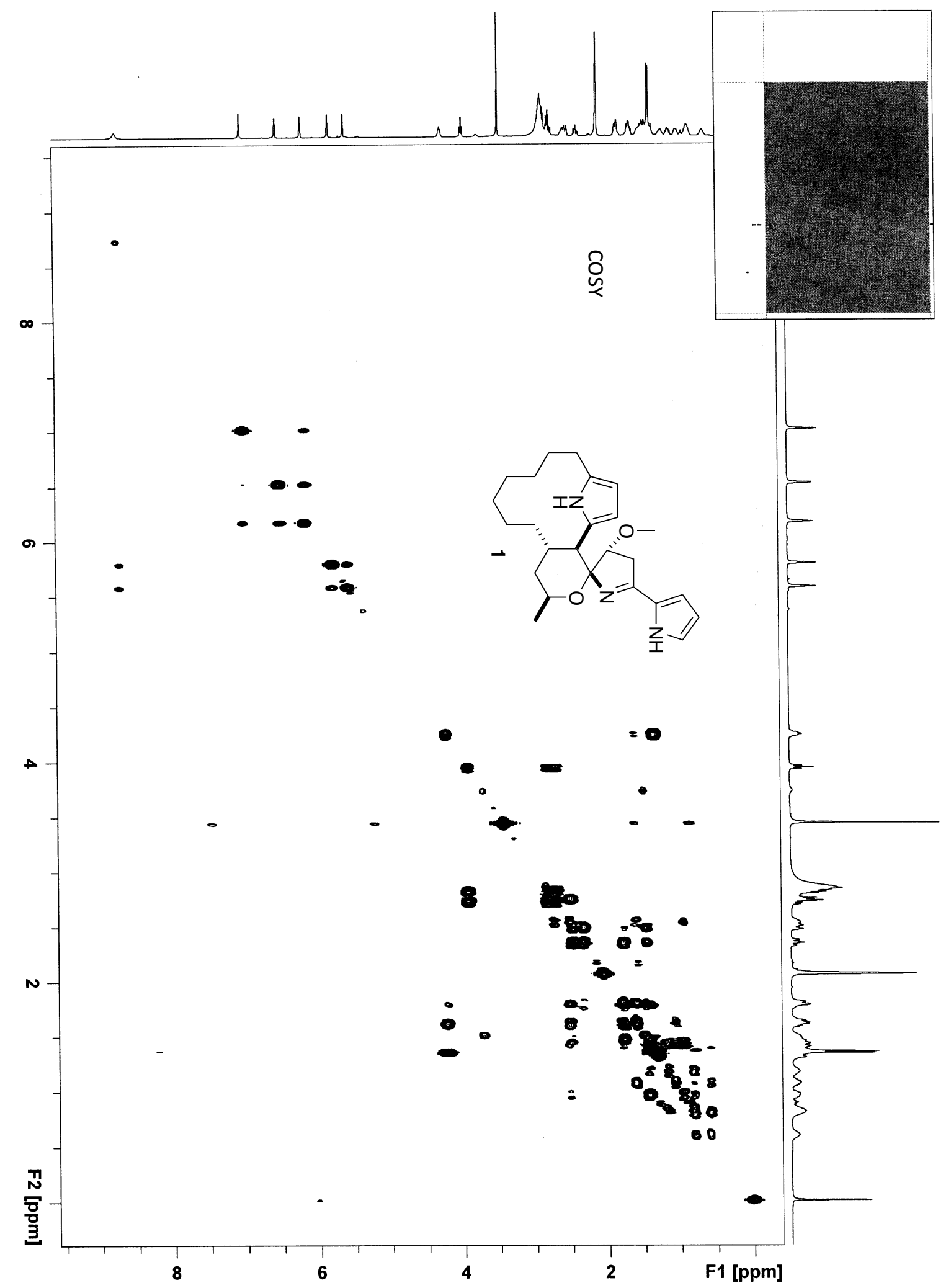

S-119 


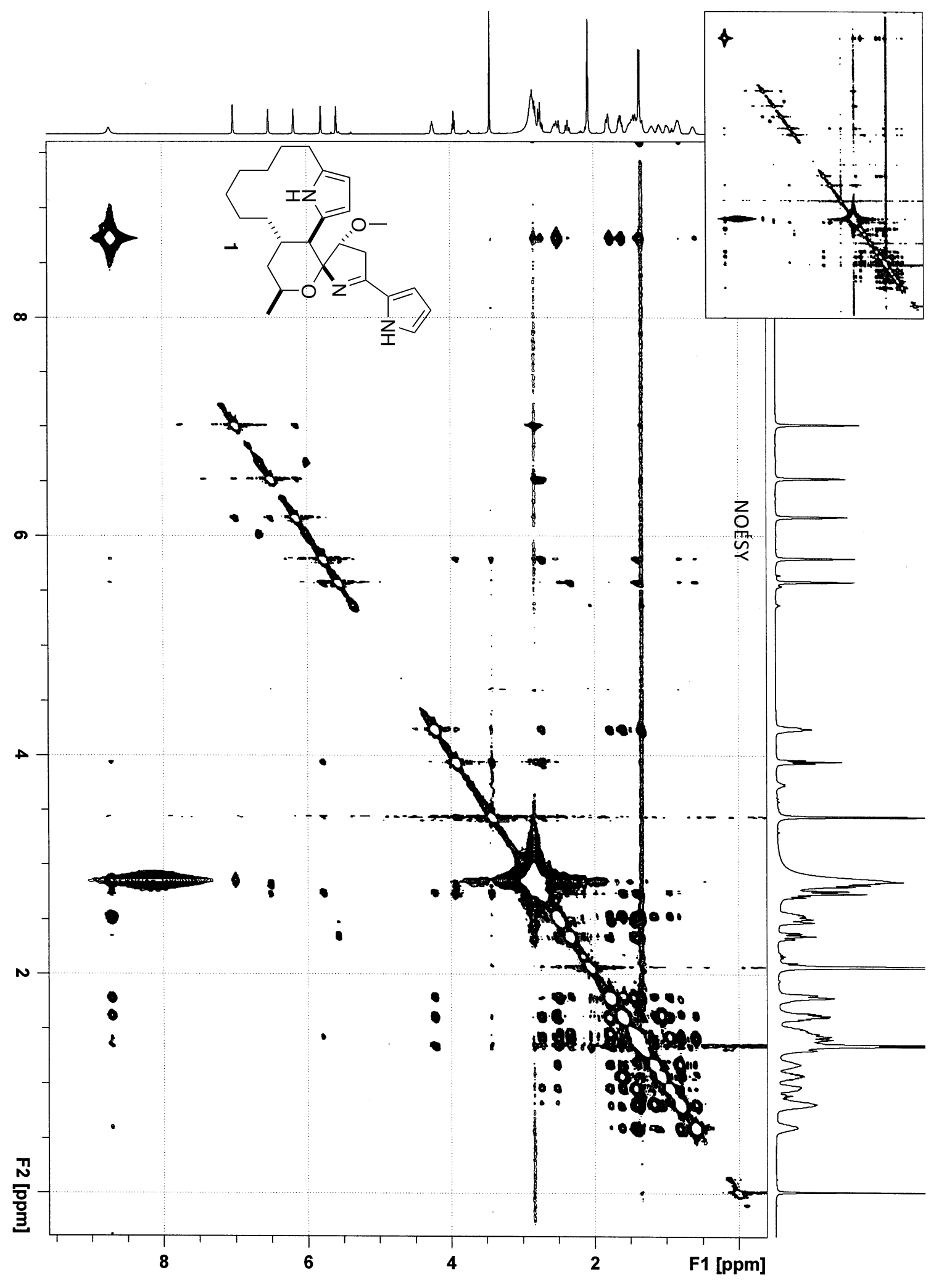


Comparison of ${ }^{1} \mathrm{H}$ NMR spectra of natural and synthetic compounds

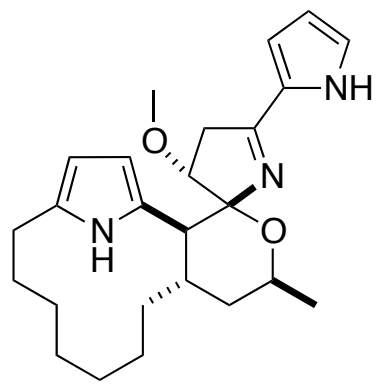

Natural compound ${ }^{\mathrm{a}}$

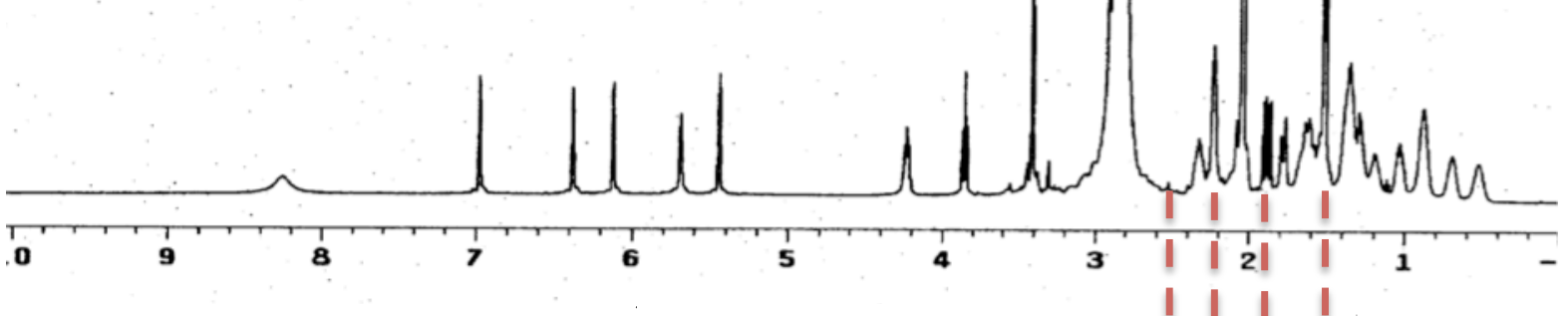

Synthetic compound

| | | |

I I I

I I I I

| | | |

I I|| I

I I | I

1 | | I I

\begin{tabular}{ll|ll|}
1 & 1 & 1 & 1
\end{tabular}

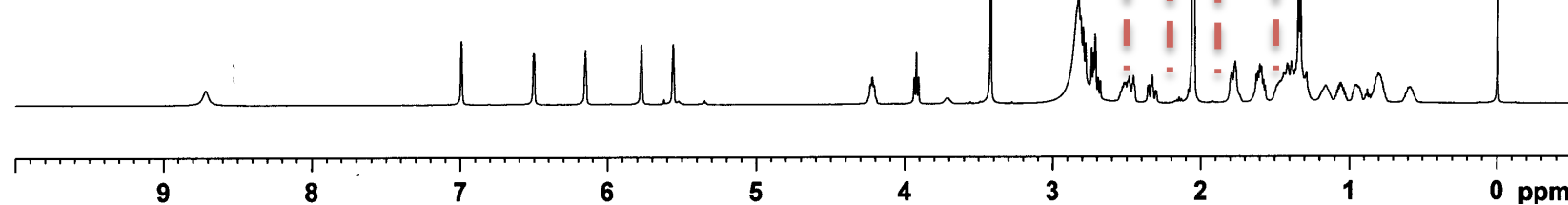

${ }^{\mathrm{a}}$ The NMR spectrum of natural compound is taken from Boonlarppradab, C.; Kauffman, C. A.; Jensen, P. R.; Fenical, W. Org. Lett. 2008, 10, 5505. 
Comparison of ${ }^{13} \mathrm{C}$ NMR spectra of natural and synthetic compounds

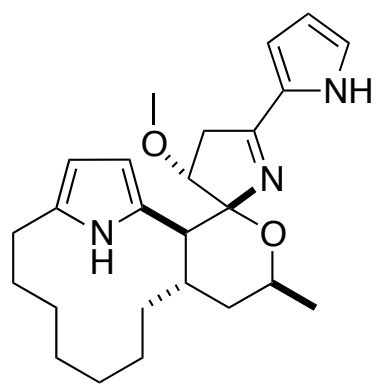

Natural compound ${ }^{\mathrm{a}}$

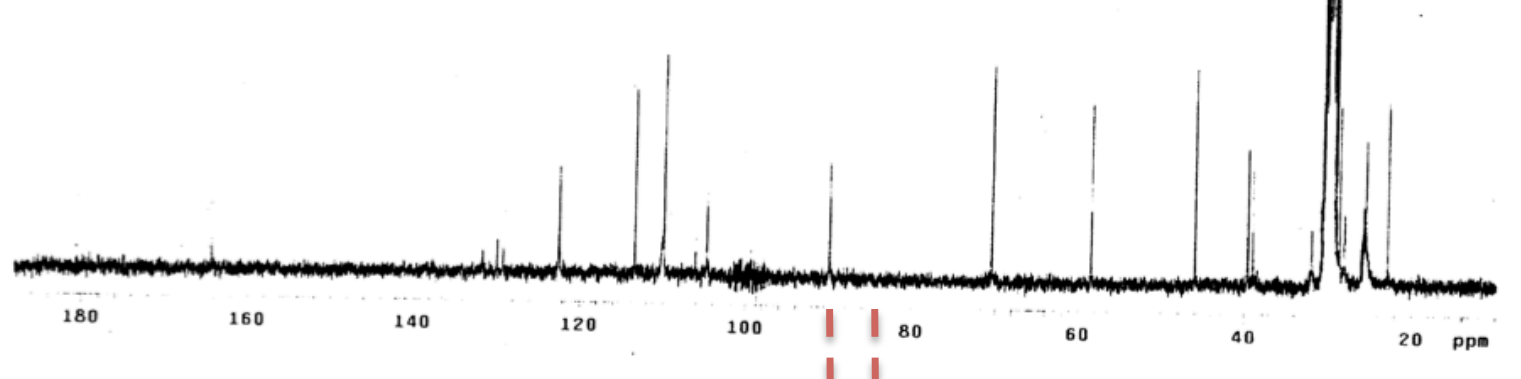

Synthetic compound
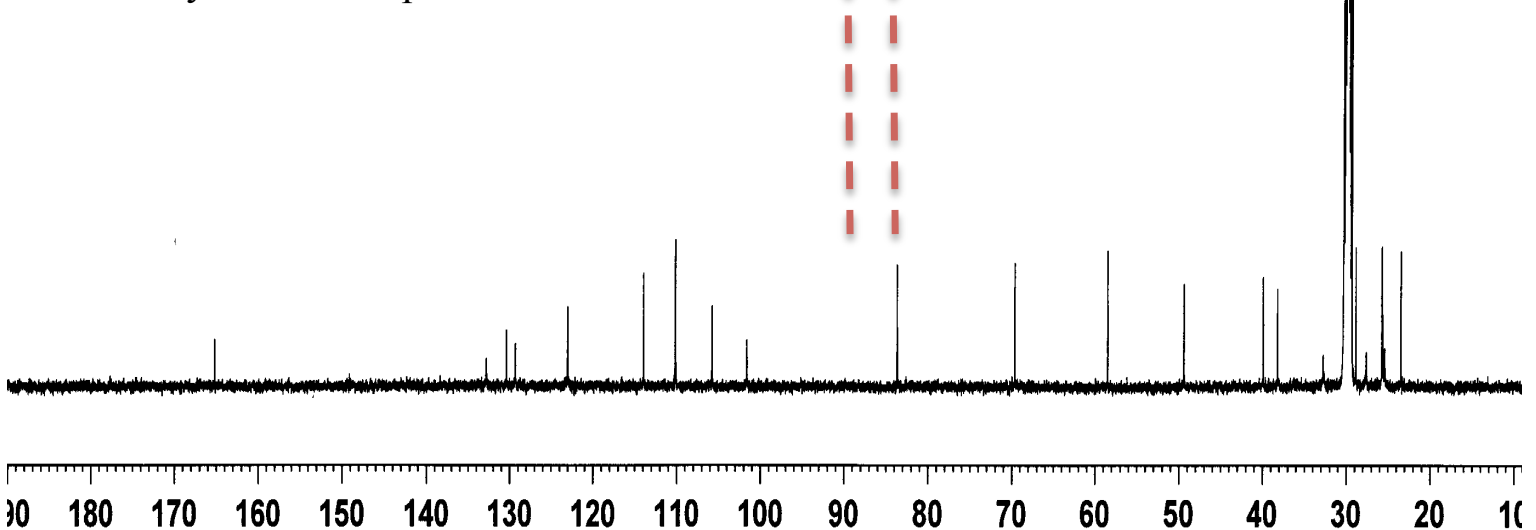

${ }^{\mathrm{a}}$ The NMR spectrum of natural compound is taken from Boonlarppradab, C.; Kauffman, C. A.; Jensen, P. R.; Fenical, W. Org. Lett. 2008, 10, 5505. 